\title{
Vergleich unterschiedlicher Anbaumethoden von Energieholzplantagen
}

\author{
Dissertation \\ zur Erlangung des Doktorgrades \\ der Fakultät für Forstwissenschaften und Waldökologie \\ der Georg-August-Universität Göttingen
}

\author{
vorgelegt von \\ Bettina Stoll \\ geboren in Oldenburg (Oldb)
}

Göttingen, 2011 
1. Gutachterin / 1. Gutachter: Prof. Dr. Achim Dohrenbusch

2. Gutachterin / 2. Gutachterin: Prof. Dr. Reiner Finkeldey

Tag der mündlichen Prüfung: 19.12.2011 


\section{Danksagung}

Zu erst möchte ich meinem Betreuer und Themensteller Prof. Dr. Achim Dohrenbusch für die Möglichkeit zur Promotion danken. Ebenso möchte ich den allen Projektteilnehmern für die gute Zusammenarbeit während der Projektdauer danken, insbesondere dem Projektkoordinator Prof. Dr. Norbert Lamersdorf und den Mitarbeitern der Stadtwerke Gütersloh Martin Schmalenstroer und Dr. Mechthild Meier.

Den Mitarbeitern der Abteilung Waldbau der gemäßigten Zonen gilt mein Dank für das gute Arbeitsklima und die Unterstützung bei der praktischen Durchführung der Versuche. Hierbei waren vor allem Heiko Rubbert und Andrea Bauer eine große Unterstützung. Auch Meike Kirscht möchte ich danken, die mir insbesondere in der Anfangsphase viele wertvolle Tipps gegeben hat.

Außerdem danke ich herzlich meiner Familie und meinen Freunden bei der moralischen Unterstützung während der Arbeit. Insbesondere Theresa Waldmann, Daniela Kempa, Jessica Preutenborbeck und Andreas Mölder waren mir eine große Hilfe während dieser Zeit. Dominik Widmann und Annika Seiler haben mich besonders in der Endphase der Arbeit motiviert, am Ball zu bleiben.

Auch allen nicht namentlich erwähnten möchte ich hiermit einen großen Dank aussprechen. Ohne die Unterstützung Euch aller, hätte ich es wahrscheinlich nie soweit geschafft. Danke! 


\section{Inhaltsverzeichnis}

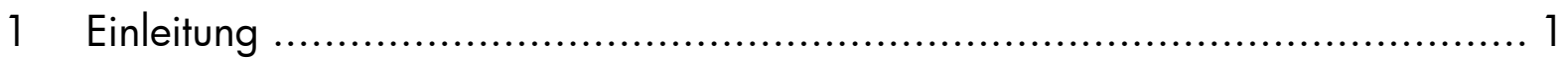

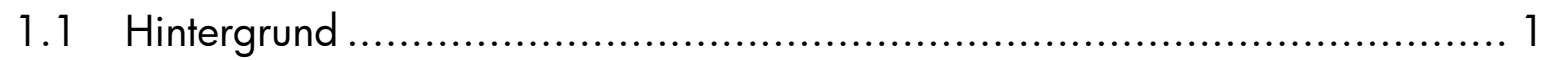

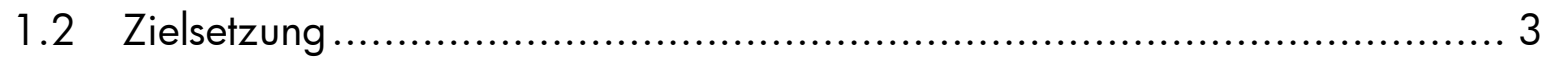

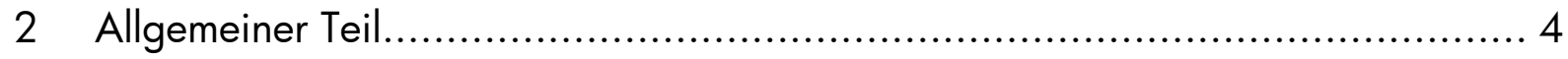

2.1 Baumarten für Kurzumtriebsplantagen ............................................. 4

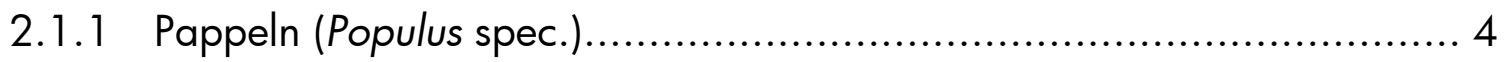

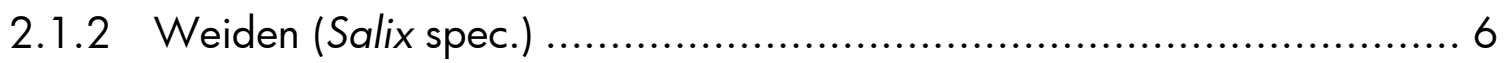

2.1.3 Robinie (Robinia pseudoacacia) ................................................. 7

2.1.4 Bergahorn (Acer pseudoplatanus) ............................................. 8

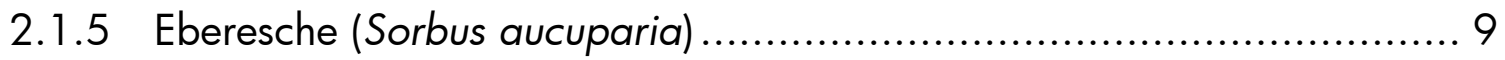

2.1.6 Erlen (Alnus spec.) ............................................................. 10

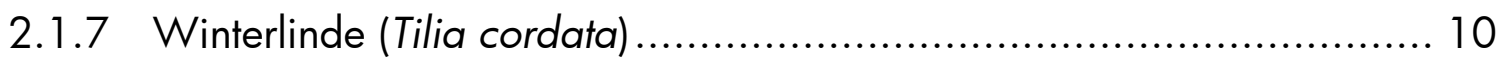

2.2 Standortanforderungen ................................................................. 11

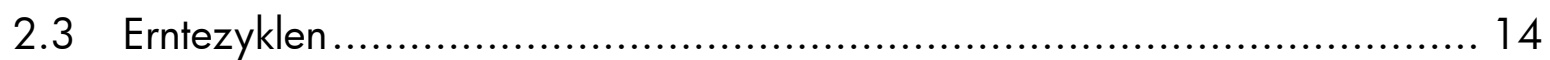

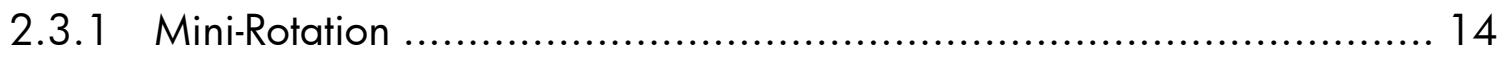

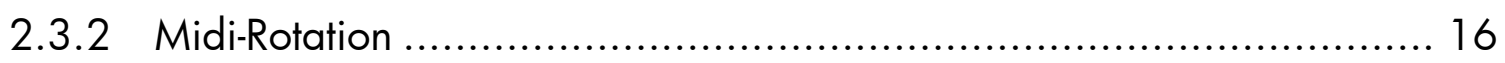

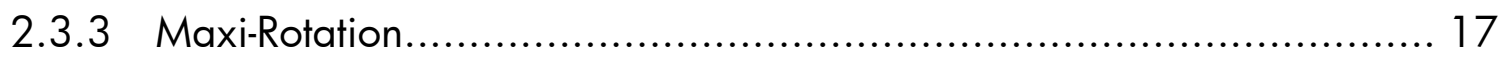

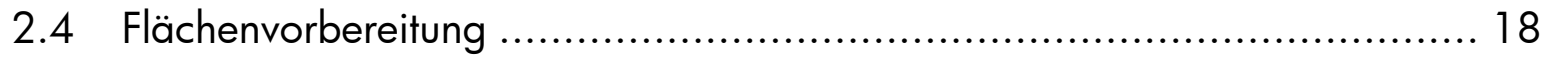

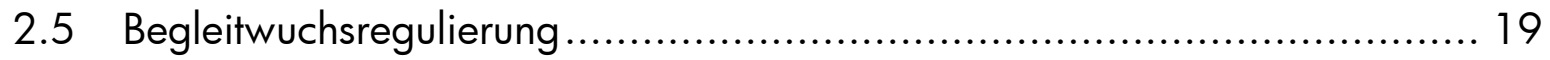

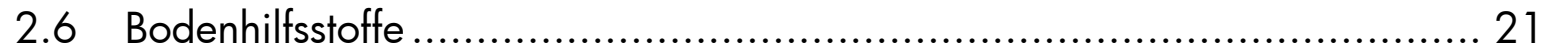

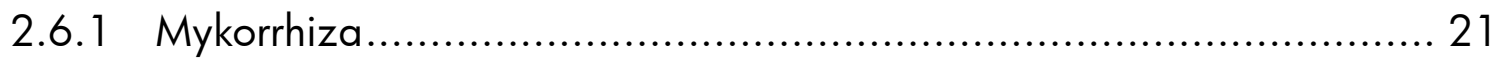

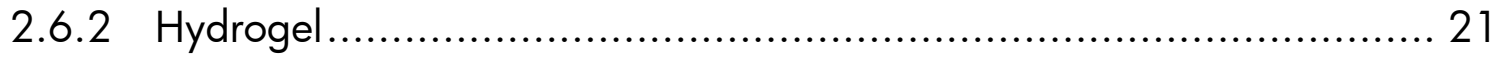

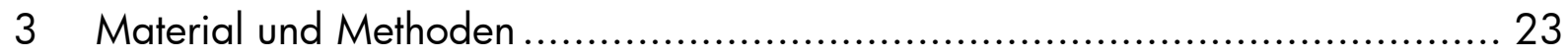

3.1 Versuchsfläche Sudheide (Landkreis Gütersloh)................................... 23 


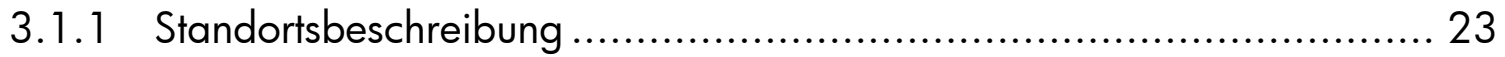

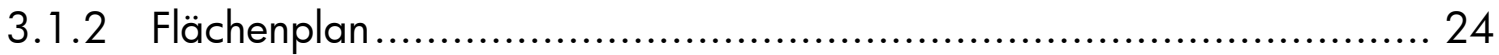

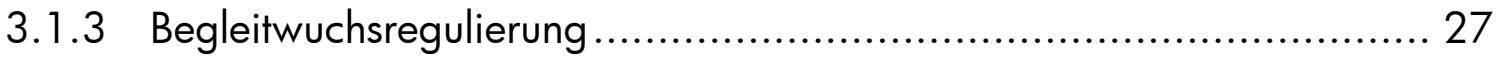

3.1.4 Hilfsstoffe (Mykorrhiza/Hydrogel)............................................... 29

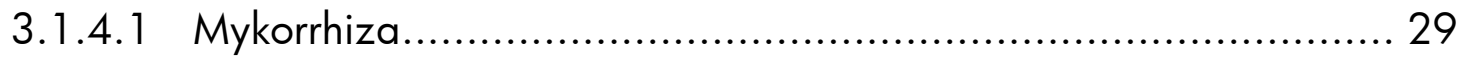

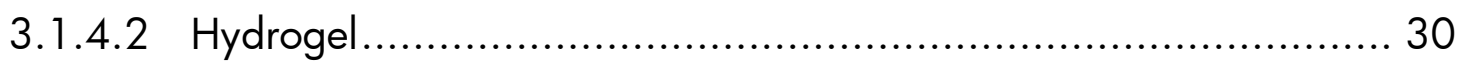

3.2 Versuchsfläche Hamerstorf (Landkreis Uelzen) ...................................... 30

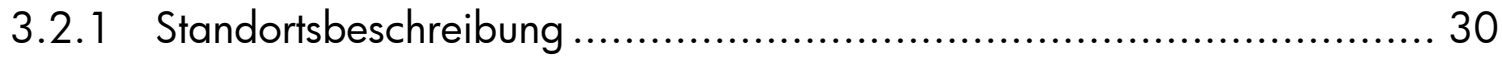

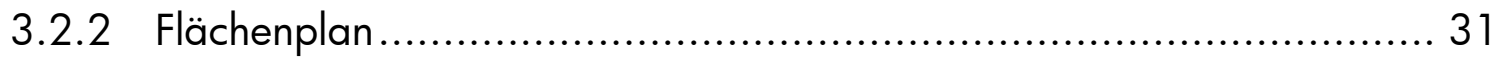

3.3 Versuchsfläche Unterlüß (Landkreis Uelzen) ........................................ 33

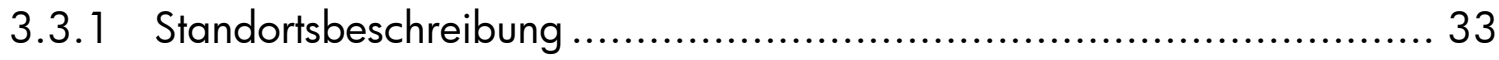

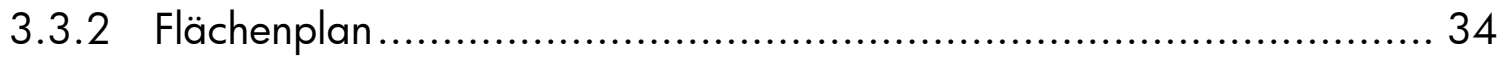

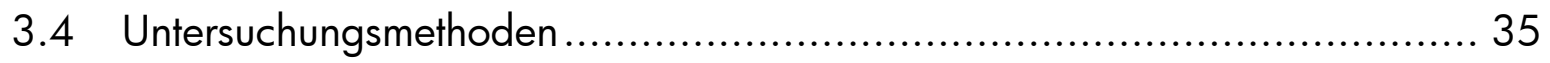

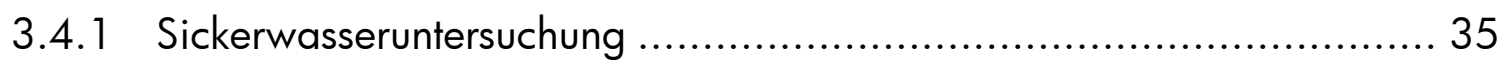

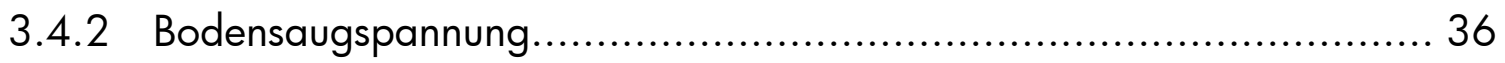

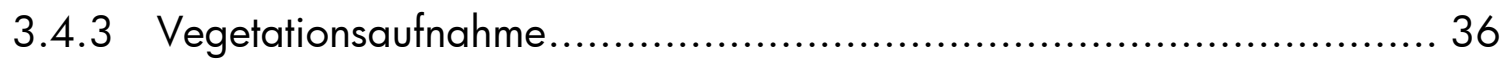

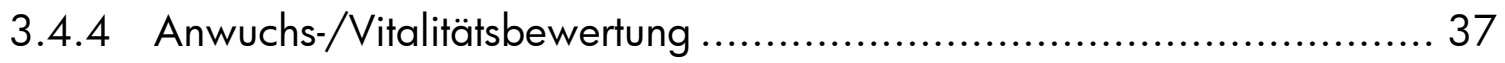

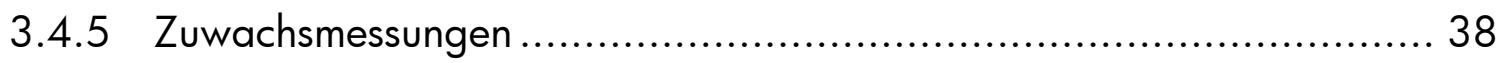

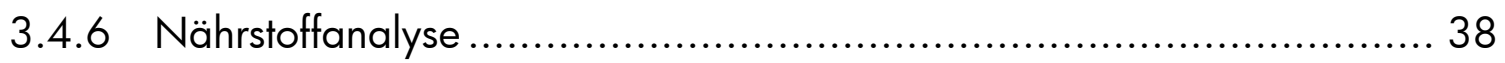

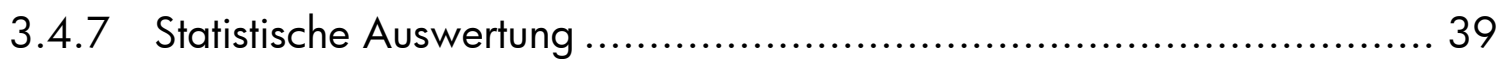

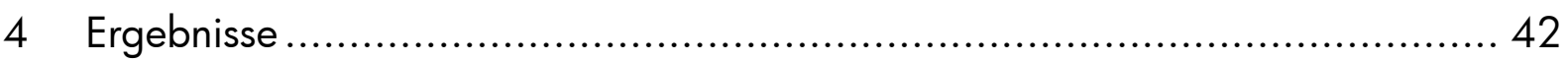

4.1 Versuchsfläche Sudheide (Landkreis Gütersloh)................................. 42

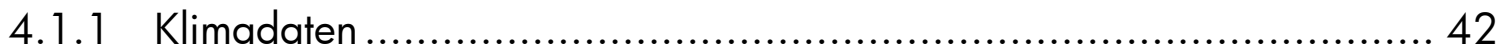

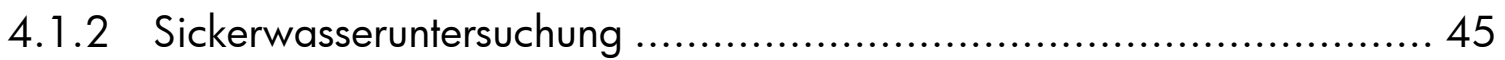

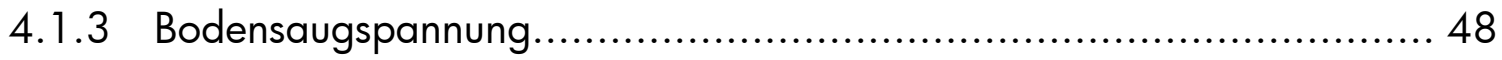

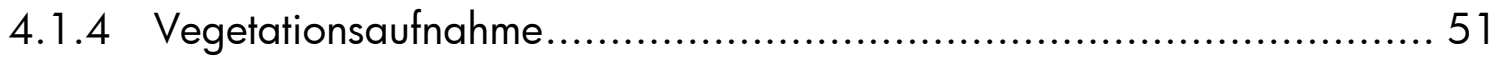




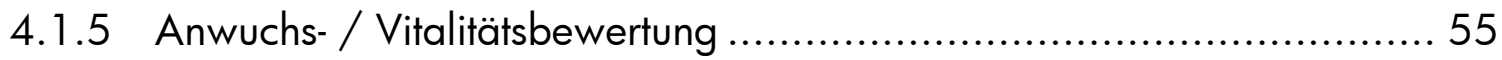

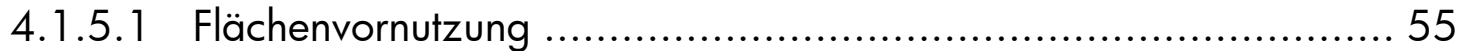

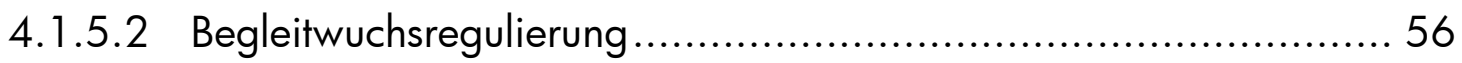

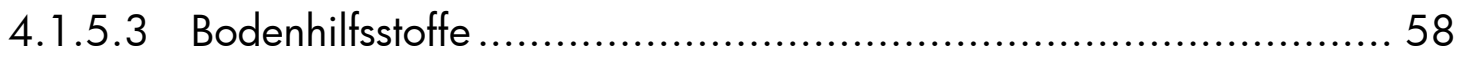

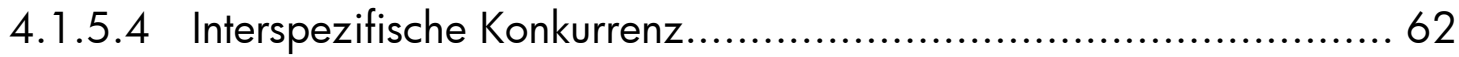

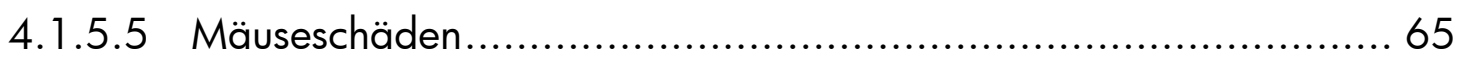

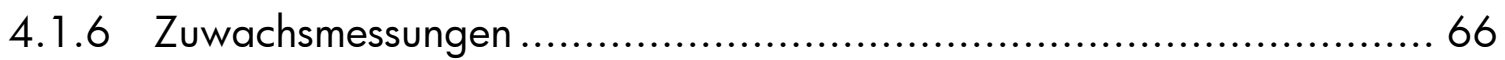

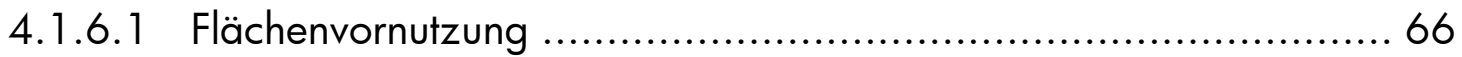

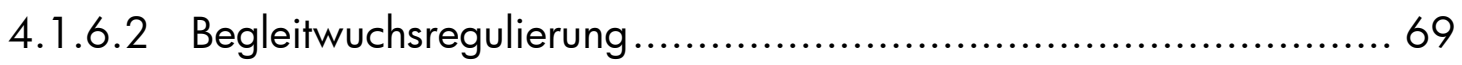

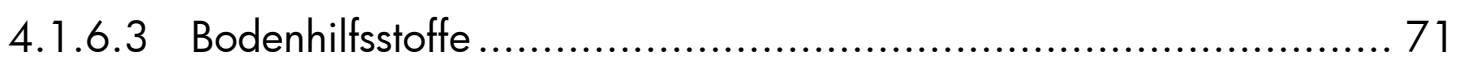

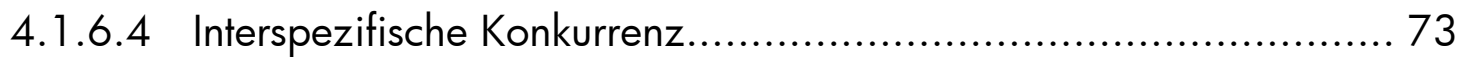

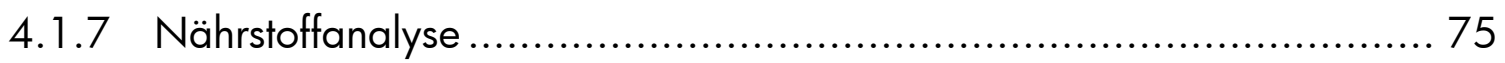

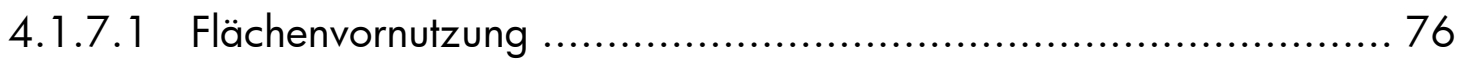

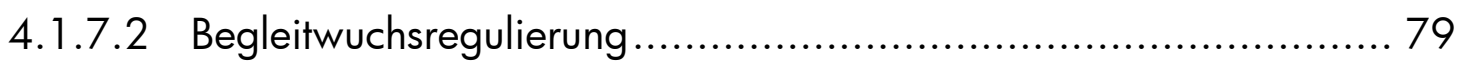

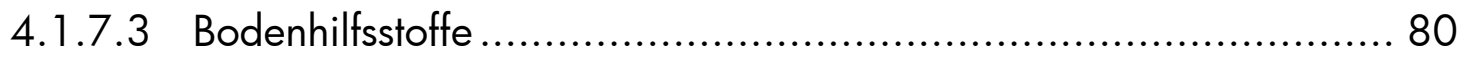

4.2 Versuchsfläche Hamerstorf (Landkreis Uelzen) ..................................... 82

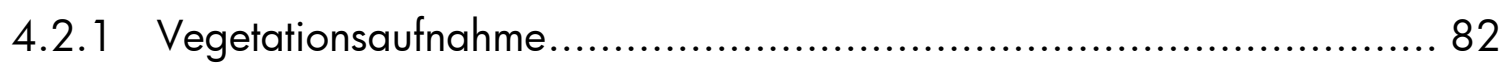

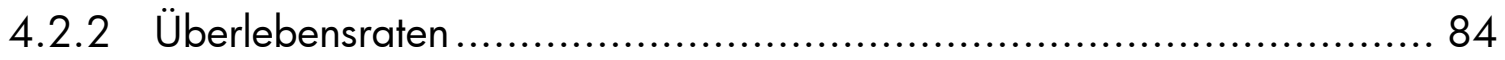

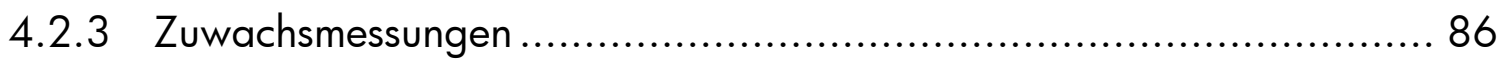

4.3 Versuchsfläche Unterlüß (Landkreis Uelzen) ........................................ 88

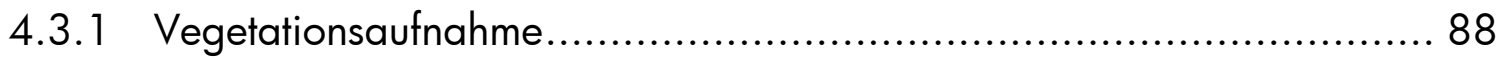

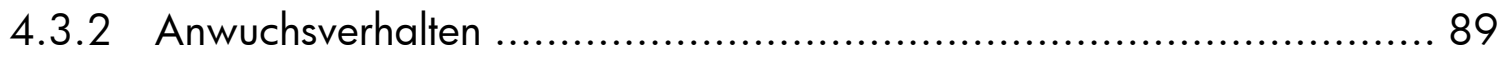

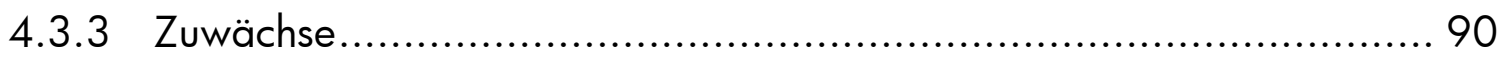

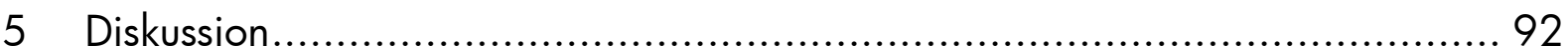

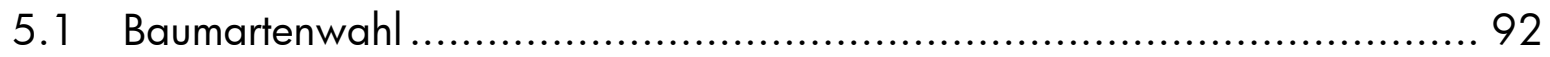


5.2 Flächenvornutzung ....................................................... 95

5.3 Flächenvorbereitung ....................................................... 98

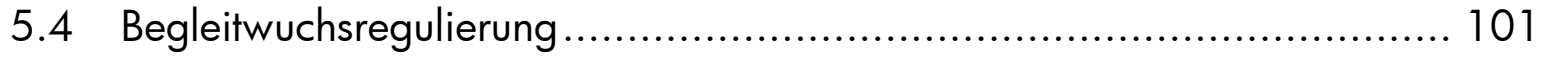

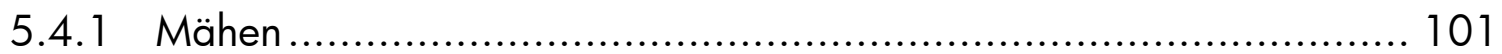

5.4.2 Nutzpflanzen...................................................... 104

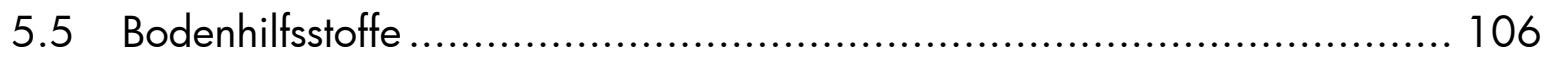

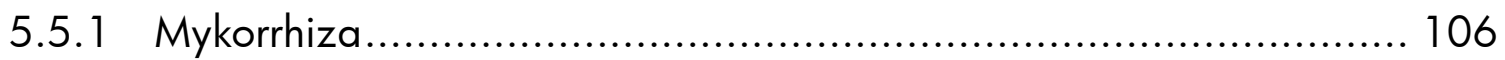

5.5.2 Hydrogel........................................................... 110

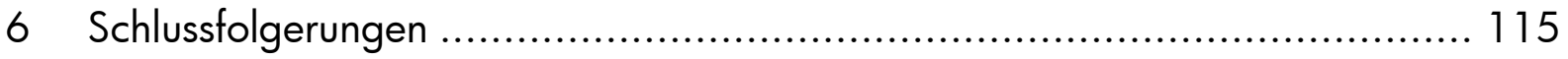

7 Zusammenfassung .......................................................... 118

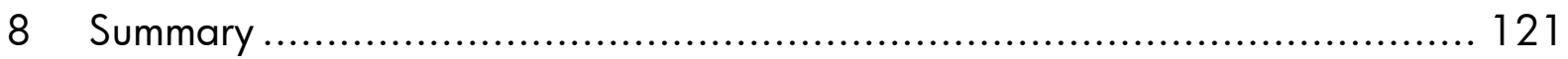

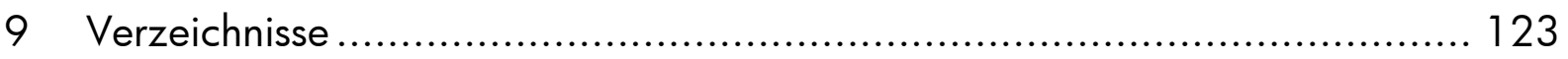

9.1 Abbildungen............................................................... 123

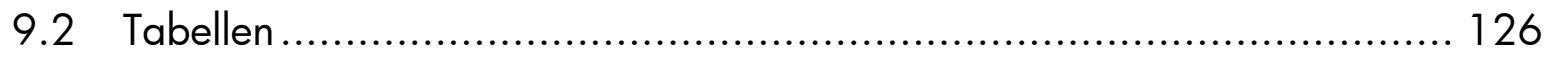

9.3 Datenquellen ................................................................. 128

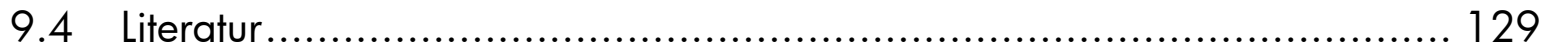




\section{Einleitung}

\subsection{Hintergrund}

In Zeiten nachhaltig steigender Preise für fossile Energieträger nimmt der Bedarf an Energieholz deutlich zu. Allein durch die Holzproduktion im Wald ist der Bedarf der stofflichen und energetischen Holzverwerter in Deutschland nicht zu decken (Landgraf et al., 2007). Eine Möglichkeit, dem zunehmenden Holzbedarf nachzukommen, stellt die Anlage von Energieholzplantagen dar. Das Potential, Acker- und Grünlandflächen im Agrarsektor mit schnellwachsenden Baumarten aufzuforsten und Holz für die Energienutzung zu erzeugen, ist ausreichend vorhanden (Thrän, 2005). Nun sind Anleitungen für eine erfolgreiche und gleichzeitig umweltgerechte Flächenanlage gefragt.

Insbesondere der Konkurrenzdruck der Begleitvegetation ist ein Faktor, der zum Scheitern des Vorhabens führen kann. Die zuvor von der Landwirtschaft bewirtschafteten Flächen sind äußerst fruchtbar verglichen mit traditionellen Forststandorten und sind im Zusammenhang mit den vorhandenen Samenbanken geradezu prädestiniert für einen üppigen und raschen Unkrautbewuchs (Willoughby \& McDonald, 1999). Da die aufkommende bzw. vorhandene Feldvegetation mit den Gehölzen insbesondere um Wasser, Nährstoffe und Licht konkurriert, sind wirkungsvolle Methoden der Begleitwuchsregulierung zu ergreifen. Wie intensiv die Unkrautbekämpfung zu betreiben ist, hängt stark von der Flächenvornutzung und dem verwendeten Arten- und Sortenmaterial ab. Größeres Pflanzenmaterial benötigt eine wesentlich eingeschränktere Bodenbearbeitung als kleineres Material wie Stecklinge, da größere Pflanzen weniger durch Unkraut gefährdet werden (Diederichs, 1990). Außerdem unterscheiden sich verschiedene Baumarten beträchtlich in ihrer ökologischen Charakteristik, so dass spezifische Maßnahmen zur Begleitwuchsregulierung zu treffen sind (Hytönen \& Jylhä, 2005).

Eine effektive Flächenbehandlung stellt die Ausbringung von Herbiziden dar. Aus ökologischen Gründen wurde bei der Flächenanlage der Einsatz von Herbiziden auf 
ein Minimum reduziert und alternative Verfahren angewendet. Eine Alternative zur chemischen Unkrautbekämpfung ist zum einen die mechanische Entfernung der Begleitvegetation. Durch Mähen zwischen den Pflanzen wird die Begleitvegetation zunächst klein gehalten, so dass die jungen Forstpflanzen weniger um Licht konkurrieren müssen. Eine andere Alternative ist die Einsaat einer Nutzpflanzendecke, die in Untersuchungen von Denecke (1988) und Reinecke (1990) das Aufkommen unerwünschter Unkräuter verhindern konnte, ohne selber die Gehölze in ihrer Wuchsentwicklung zu hemmen.

Um die Startbedingungen der neu gepflanzten Bäume zusätzlich zu verbessern und den Kulturerfolg zu erhöhen, können den Pflanzen Bodenhilfsstoffe wie Mykorrhiza und Hydrogel beigegeben werden. Die Kolonisation mit Mykorrhiza-Pilzen kann den frühen Zuwachs und die Vitalität der Bäume erhöhen (Baum et al., 2000), da die Symbiose der Pflanzen mit den Mykorrhiza-Pilzen die Wasser- und Nährstoffversorgung verbessern und der Pilzmantel der Ektomykorrhiza die Wurzeln gegen Pathogene schützen kann (Smith \& Read, 1997). Aber auf Flächen, die lange landwirtschaftlich genutzł wurden, ist das Potenzial von Mykorrhiza-Pilzen gering, da die passenden Wirte fehlen (Berman \& Bledsoe, 1998). Bei Aufforstungen von landwirtschaftlichen Flächen kann das geringe Aufkommen von Mykorrhiza-Pilzen einen hohen Ausfall der neu gepflanzten Bäume nach sich ziehen (Marx et al., 2002), weshalb eine Ansiedlung von Mykorrhiza-Pilzen bei der Pflanzung sinnvoll sein kann. Neben der Verfügbarkeit von Mykorrhiza-Pilzen, wird der Kulturerfolg vor allem von den Bodeneigenschaften und der Wasserverfügbarkeit beeinflusst. Deshalb ist es sinnvoll neve oder modifizierte Produkte zur Verbesserung der Wasserspeicherung zu testen. Hydrogele sind hochvernetzte Polyacrylamide, welche in der Lage sind das 400-fache ihres Eigengewichtes an Wasser zu absorbieren und damit in Trockenperioden den Wasserstress für die Pflanzen zu reduzieren. 


\subsection{Zielsetzung}

Ziel dieser Arbeit ist es, ist den Einfluss der Flächenvornutzung auf verschiedene Gehölze zu bewerten und Hinweise zu effektiven und umweltgerechten Kulturpflegemaßnahmen von Energieholzplantagen geben zu können.

- Quantifizierung der Entwicklung von Aufforstungen mit schnellwachsenden und alternativen Baumarten auf unterschiedlichen Standorten.

- Quantifizierung der Wirkung von Bodenhilfsstoffen wie Mykorrhiza und Hydrogel auf die Vitalität und Wuchsentwicklung von unterschiedlichen Baumarten.

- Erfassung der ökologischen Wirkung von unterschiedlichen Aufforstungstypen auf naturschutzrelevante Teile des Ökosystems in den ersten Vegetationsperioden (Bodenvegetation, Sickerwasserqualität, Nährstoffversorgung).

- Herleitung von Empfehlungen zur Anlage und Behandlung von Energieholzkulturen in den ersten Vegetationsperioden. 


\section{Allgemeiner Teil}

\subsection{Baumarten für Kurzumtriebsplantagen}

Für die Produktion von Energieholz werden Baumarten eingesetzt, die dem Produktionsziel und den standörtlichen Produktionsbedingungen optimal angepasst sind. Geeignet dafür sind Lichtbaumarten mit einer raschen Jugendwüchsigkeit. Zusätzlich sind Merkmale, wie eine hohe Früh- und Spätfrostresistenz, sicheres Anwuchsverhalten und eine hohe Konkurrenzverträglichkeit gegen Dichtstand von Vorteil. Die vegetative Vermehrbarkeit des Pflanzgutes hat den Vorteil, die Anlagenkosten durch die Verwendung von Stecklingen und eine gute Wiederausschlagsfähigkeit nach der Ernte gering zu halten. Eine geringe Krankheitsanfälligkeit, hohe Toleranz gegen Schadinsekten und geringe Wildverbissgefährdung reduzieren die Ausfallquote und erhöhen den Biomasseertrag von Kurzumtriebsplantagen.

\subsubsection{Pappeln (Populus spec.)}

Pappeln sind mit ca. 40 Arten in den gemäßigten Klimazonen der gesamten Nordhalbkugel vertreten. Aufgrund natürlicher Hybridisierung zeigen sie einen großen Formenreichtum. Ihr Vorkommensgebiet wird durch eine weite ökologische Standortsamplitude gekennzeichnet (Traupmann, 2004). Ein weiteres wichtiges Merkmal ist ihre starke Wuchs- und Ertragsleistung in den ersten zehn Jahren ihrer Entwicklung. Die Eigenschaften von wichtigen Pappelarten nach Röhricht \& Ruscher (2004) sind in Tab. 2.1 aufgelistet. 
Tab. 2.1: Eigenschaften von wichtigen Pappelarten für den Kurzumtrieb (Röhricht \& Ruscher, 2004)

\begin{tabular}{|c|c|c|c|}
\hline $\begin{array}{l}\text { Familie } \\
\text { Gattung }\end{array}$ & $\begin{array}{l}\text { Salicaea - Weidengewächse } \\
\text { Populus }\end{array}$ & & \\
\hline Sektion & $\begin{array}{l}\text { AIGEIROS } \\
\text { (Schwarzpappeln) }\end{array}$ & $\begin{array}{l}\text { TACAMAHACA } \\
\text { (Balsampappeln) }\end{array}$ & $\begin{array}{l}\text { LEUCE } \\
\text { (Weiß-/Zitterpappeln) }\end{array}$ \\
\hline $\begin{array}{l}\text { Natürliche } \\
\text { Verbreitung }\end{array}$ & $\begin{array}{l}\text { Europa, W-Asien, NW- } \\
\text { Afrika, N-Amerika }\end{array}$ & N-Amerika, Asien & $\begin{array}{l}\text { Europa, Asien, N- } \\
\text { Amerika, N-Afrika }\end{array}$ \\
\hline Wichtige & P. nigra (Europäische & P. trichocarpa & P. alba (Weißpappel) \\
\hline Arten & $\begin{array}{l}\text { Schwarzpappel) } \\
\text { P. deltoides } \\
\text { (Amerikanische } \\
\text { Schwarzpappel) }\end{array}$ & $\begin{array}{l}\text { (Amerikanische } \\
\text { Balsampappel) } \\
\text { P. maximowiczii } \\
\text { (Asiatische Balsampappel) } \\
\text { P. balsamifera } \\
\text { (Nordamerikanische } \\
\text { Balsampappel) }\end{array}$ & $\begin{array}{l}\text { P. tremula (Europäische } \\
\text { Aspe) } \\
\text { P. tremuloides } \\
\text { (Amerikanische Aspe) }\end{array}$ \\
\hline $\begin{array}{l}\text { Standort- } \\
\text { ansprüche }\end{array}$ & $\begin{array}{l}\text { Hohe Ansprüche an } \\
\text { Nährstoffversorgung und } \\
\text { Durchwurzelbarkeit, keine } \\
\text { Staunässe }\end{array}$ & $\begin{array}{l}\text { Wechselfeuchte Standorte, } \\
\text { keine windexponierten } \\
\text { Lagen }\end{array}$ & $\begin{array}{l}\text { Mäßig nasse bis trockene } \\
\text { Standorte, jedoch } \\
\text { Grundwassereinfluss, } \\
\text { geringe Ansprüche an } \\
\text { Klima und Standort }\end{array}$ \\
\hline Bodenart & $\begin{array}{l}\text { Bevorzugt Aueböden, gut } \\
\text { durchlüftete Standorte, } \\
\text { gute Wasserführung, hohe } \\
\text { pH-Werte }\end{array}$ & $\begin{array}{l}\text { Breites Spektrum: } \\
\text { Sandböden bis Lehm, } \\
\text { auch trockene Standorte, } \\
\text { optimal fließendes } \\
\text { Grundwasser 1- } 2 \text { m, } \\
\text { nährstoffreich, kalkhaltig }\end{array}$ & $\begin{array}{l}\text { Breites Spektrum: } \\
\text { Kippenböden bis schwere } \\
\text { Lehmböden, optimal sind } \\
\text { frische humus- und } \\
\text { nährstoffreiche Böden }\end{array}$ \\
\hline $\begin{array}{l}\text { Wuchs- und } \\
\text { Ertragsleistung }\end{array}$ & Empfindlich auf Dichtstand & Rasches Jugendwachstum & $\begin{array}{l}\text { Starkes Jugendwachstum, } \\
\text { gute Verträglichkeit des } \\
\text { "auf den Stocksetzen", } \\
\text { Dichtstandverträglichkeit }\end{array}$ \\
\hline $\begin{array}{l}\text { Wichtige } \\
\text { Schädlinge }\end{array}$ & - & $\begin{array}{l}\text { Gute Resistenz gegen } \\
\text { Pappelbock und } \\
\text { Pappelblattkäfer }\end{array}$ & $\begin{array}{l}\text { Kleiner und großer } \\
\text { Pappelbock, roter } \\
\text { Pappelblattkäfer }\end{array}$ \\
\hline Besonderheit & $\begin{array}{l}\text { Nicht geeignet für } \\
\text { Kurzumtrieb, große } \\
\text { Bedeutung als } \\
\text { Kreuzungspartner }\end{array}$ & $\begin{array}{l}\text { Dichtstandverträglichkeit, } \\
\text { kräftige Bewurzelung }\end{array}$ & $\begin{array}{l}\text { Keine } \\
\text { Stecklingsbewurzelung, } \\
\text { langsames Wachstum ( } P \text {. } \\
\text { tremula), mind. 8-jähriger } \\
\text { Umtrieb notwendig }\end{array}$ \\
\hline
\end{tabular}

Von Hofmann (2007) wurde eine Übersicht über die Biomasseleistungen der wichtigsten Pappelkreuzungen bei einer Umtriebszeit von 4 Jahren mit 16.667 Pflanzen/ha und guten Wuchsbedingungen gegeben (Tab. 2.2). 
Tab. 2.2: Biomasseleistung der wichtigsten Pappelarten und Kreuzungen bei einer Umtriebszeit von vier Jahren und guten Wuchsbedingungen (Hofmann, 2007)

\begin{tabular}{l|ll}
\hline Kreuzungskombination & $\begin{array}{l}\text { 1. Rotation } \\
{[\mathbf{t} / \mathbf{h a} / \mathbf{a}]}\end{array}$ & $\begin{array}{l}\text { 2. Rotation } \\
{[\mathbf{t} / \mathbf{h a} / \mathbf{a}]}\end{array}$ \\
\hline P. trichocarpa & 8,7 & 18,4 \\
P. trichocarpa $x$ P. deltoides & 11,5 & 19,9 \\
P. nigra P. maximowiczii & 10,8 & 17,3 \\
P. x euramericana & 7,2 & 6,2 \\
P. tremula $x$ P. tremuloides & 11 & 4,7 \\
\hline
\end{tabular}

Unter weniger guten Bedingungen, wie einem lehmig sandigen Substrat in der Bergbau-Region Welzow-Süd in Brandenburg, wurde von Pappelhybriden eine geringere Trockenbiomasseleistung von $24-49 t$ innerhalb von 8 Jahren erreicht (Bungart \& Huttl, 2004), was einem jährlichen Zuwachs von 3-6 t/ha entspricht. In Bayern haben Balsampappelklone im Vergleich mit Schwarzerle, Robinie und Weide die höchste Biomasseleistung von $13 \mathrm{t} / \mathrm{ha} / \mathrm{a}$ erreicht, was in etwa einem Brennwert von 5000 I Heizöl entspricht (Burger, 2006).

\subsubsection{Weiden (Salix spec.)}

Weiden gehören mit ca. 400-500 verschiedenen Arten (Liebhard, 2007) zu einer der formenreichsten Gehölzgattungen der Welt. Die Neigung zu natürlicher Hybridisierung nahverwandter Arten ist sehr ausgeprägt (Traupmann, 2004). Zum Energieholzanbau eigenen sich Weiden aufgrund ihrer hohen Anwuchssicherheit als Steckling, ihrer ausgeprägten Fähigkeit zum Stockausschlag sowie der geringen Anfälligkeit gegen Schäden. Weiden sind raschwüchsig, allerdings relativ kurzlebig. Aufgrund ihres hohen Lichtanspruches sind Weiden konkurrenzschwach.

Als Energiewaldarten kommen laut Traupmann (2004) meist nur die sehr leistungsfähigen Sorten von Hochstrauchformen wie Korbweide (S. viminalis), Kätzchenweide (S. x smithiana) und Filzastweide (S. dasyclados) in Frage. Maximale Leistungen werden dabei auf frisch-(wechsel-) feuchten, nährstoffreichen sandigen Lehmen erbracht. Raschwüchsige Sorten weisen oftmals geringere Salicingehalte in der Rinde auf und werden daher vom Rehwild bevorzugt verbissen (Traupmann, 2004). 
Tab. 2.3: Eigenschaften von Weidenarten für den Kurzumtrieb (Röhricht \& Ruscher, 2004)

\begin{tabular}{l|l}
\hline Familie & Salicaea - Weidengewächse \\
Gattung & Salix \\
Wichtige Arten & S. viminalis (Korb-/Hanfweide) \\
& S. smithiania (Kätzchenweide) \\
Standortansprüche & S. dasyclados (Filzastweide) \\
Bodenart & Brechselfeuchte bis feuchte Standorte, jedoch keine Staunässe \\
Wuchs- und & Starkes Jugendwachstum \\
Ertragsleistung & \\
Wichtige Schädlinge & Weidenblattrost (Melampsora spec.) \\
Besonderheit & Dichtstandverträglichkeit, große Frosthärte \\
\hline
\end{tabular}

Die Vorzüge der Weide (Salix spec.) liegen im nahezu 100\%igen Anwuchs- und Regenerationserfolg sowie in ihrer Frosthärte. Die Ertragsleistung der Weide liegt im Allgemeinen jedoch niedriger als bei der Balsampappeln. In Umtriebszeiten bis maximal 4 Jahre werden auf leichten Böden und bei guter Wasserversorgung ca. $8 \mathrm{t} / \mathrm{ha} / \mathrm{a}$ produziert (Hofmann, 1998). Von Guericke (2006) wurde eine Trockenbiomasseproduktion zwischen $2,8 \mathrm{t} /$ ha und $6,1 \mathrm{t} /$ ha vier verschiedener schwedischer Weidenklone im ersten Standjahr gemessen. Der optimale Ernterhythmus der Weide liegt bei 3 bis 5 Jahren (Guericke, 2006).

\subsubsection{Robinie (Robinia pseudoacacia)}

Hervorragende Wuchs- und Holzeigenschaften prädestinieren die Robinie (Robinia pseudoacacia) für hochwertige Verwendungen im Außenbereich und als Energieholz in Kurzumtriebsplantagen.

Die Robinie hat eine stattliche Zuwachsleistung, einen sehr geringen Feuchtegehalt des Holzes, eine hohe Wiederaustriebfähigkeit und eignet sich für die unterschiedlichsten Standorte. Die höchsten Erträge sind dabei nur auf guten Standorten zu erwarten. Die Fähigkeit der Robinie, Luftstickstoff zu binden, ermöglicht ihr aber auch auf Extremstandorten und auf landwirtschaftlich weniger geeigneten Flächen ein passables Wachstum (Führer, 2005). Der jährliche Zuwachs liegt zwischen 6 und 11 t/ha. Das 
entspricht im besten Fall einem Brennwert von 4200 I Heizöl, im schlechtesten Fall von nur 2200 I (Schüler et al., 2006).

Nachteilig kann sich die Empfindlichkeit der Robinie gegenüber Frost auswirken. Besonders Frühfröste können große Schäden anrichten. Die Robinie schließt erst sehr spät im Jahr mit dem Trieb ab, so dass bei zeitig einsetzenden Frösten die oft noch unverholzten Triebspitzen erfrieren. An diesen abgestorbenen Stellen dringen dann sehr leicht holzzerstörende Pilze ein (Eisenreich, 1956).

Neuanpflanzungen sind oft sehr stark dem Wildverbiss ausgesetzt. Vor allem Hase, Kaninchen und Mäuse benagen die noch glatte Rinde junger Stämmchen. Andere Wildarten wie Rot-, Reh-, Dam- und Muffelwild verbeißen die Triebe und können stellenweise ziemlich große Schäden anrichten (Eisenreich, 1956).

Von besonderer Bedeutung ist die Robinie für die Bienenweide; ihre Blüten liefern bis zu $200 \mathrm{~kg}$ Honig je Hektar und Jahr (Landesanstalt für Ökologie Landschaftsentwicklung und Forstplanung NRW, 1984). Die nährstoffreiche, rasch zersetzende Streu macht sie zu einer bodenpfleglichen Holzart. Außerdem gilt die Robinie als besonders ökonomischer Wasserverbraucher.

\subsubsection{Bergahorn (Acer pseudoplatanus)}

Der Bergahorn ist auf sickerfrischen bis feuchten, nährstoff- und basenreichen lockeren Lehm oder Steinschuttböden in kühl-lufffeuchter Klimalage zu finden. Der Standort sollte mäßig saver bis alkalisch sein. Bergahorn ist verhältnismäßig resistent gegen Kälte und Wind, auch gegen salzhaltige Winde an der Küste. Spätfroste setzen ihm allerdings zu.

Junge Ahorne wachsen in der Jugend relativ schnell. Auf einem sandigen Substrat mit einem pH-Wert von 4,5 erreichte der Bergahorn in Untersuchungen von Walle et al. (2007) eine Biomasseproduktion von 1,2 t/ha/a nach einer vieriährigen Standzeit und einer Überlebensrate von $96,8 \%$. 
Der Bergahorn, der zu den wertvollsten Edellaubbäumen zählt, liefert das hellste einheimische Holz, welches vor allem bei der Anfertigung von Möbeln, im Innenausbau von Häusern und für Parkettböden verwendet. Als Zier- und Resonanzholz wird der Bergahorn zudem seit langem für den Bau von Musikinstrumenten (Streichund Blasinstrumente) genutzt. (Rabanser \& Gössinger, 2012).

Die Blätter des Bergahorn zersetzen sich zu einem sehr nährstoffreichen Humus, welcher zu einer Verbesserung der Bodeneigenschaften beiträgt. Außerdem kann die schöne goldgelbe Laubfärbung im Herbst das Landschaftsbild positiv gestalten. Ab einem Alter von etwa 30 Jahren steht der Bergahorn in voller Blüte, dient zahlreichen Insekten, Spinnen und Vogelarten als Lebensraum und Nahrungsquelle (Schoof, 2009).

\subsubsection{Eberesche (Sorbus aucuparia)}

Von dieser Baumart werden keine besonderen Ansprüche an den Standort gestellt. Die Eberesche ist anspruchslos und wächst auf fast allen Böden. Selbst auf den ärmsten Böden kann sie bei guter Lockerung ein relativ gutes Wachstum aufweisen. Die beste Entwicklung erreicht die Eberesche auf fruchtbaren, frischen und lockeren Böden mit geringem Kalkgehalt. Zur Holzproduktion ist sie auf denselben Böden wie die Sandbirke (Betula pendula) geeignet, ausgenommen armer Sande. Zusätzlich isł die Eberesche sehr frost- und spätfrosthart und verhältnismäßig resistent gegen Wind, auch gegen salzhaltige Winde an der Küste.

Neben ihrem raschen Jugendwachstum als Pionierbaumart dienen die Beeren der Eberesche im Winter vielen Vögeln und auch Säugetieren zur Nahrung. An den Boden stellt die Vogelbeere keine besonderen Ansprüche. Sie zeichnet sich besonders durch ihre Frosthärte und Winderträgnis aus. Auch Spätfroste können ihr nichts anhaben. Ihre weit reichenden Wurzeln können größere Tiefen durchdringen und die reichliche Vermehrung durch Wurzelbrut macht sie daher als Bodenschutzgehölz besonders geeignet. Die abgeworfenen Blätter zersetzen sich schnell und beeinflussen damit positiv die Humusbildung. Die dadurch bedingte bessere Nährstoffversorgung macht 
die Bäume widerstandsfähiger gegen negative Umwelteinflüsse. (Griesche \& Schmidt 2012)

\subsubsection{Erlen (Alnus spec.)}

Die Schwarzerle (Alnus glutinosa) ist für sicker- oder staunassen, z.T. zeitweise überschwemmte, nährstoffreiche, vorwiegend kalkarme Kies-, Sand-, Ton- oder Torfböden geeignet. Der Standort sollte mäßig saver bis neutral sein. Die Schwarzerle besitzł ein sehr gutes Stockausschlagvermögen und erträgt Staunässe, sehr hohe Grundwasserstände und längere Überflutungen. Traupmann (2004) gibł für die Schwarzerle Zuwächse von 6 - 8 † pro Hektar und Jahr an.

Die Grauerle (Alnus incana) ist auf sickernassen (frischen), z.T. zeitweilig überfluteten, nährstoff- und basenreich, meist kalkhaltigen, locker durchlüfteten, rohen vorwiegend kiesig-sandigen Tonböden zu finden. Der Standort sollte mäßig sauer bis alkalisch sein. Auch die Graverle besitzt ein gutes Stockausschlagvermögen. Bei einem Anbauversuch in Österreich zeigte die Graverle auf frischen bis sehr frischen Standorten und einem Pflanzverband von $1 \times 2$ m eine Biomasseproduktion von durchschnittlich 5,8 - 7,1 t/ha/a nach 6 Jahren Umtriebszeit auf (Schuster, 2007).

\subsubsection{Winterlinde ( Tilia cordata)}

Die Linde ist schnellwüchsig und kommt auf frischen bis mäßig trockenen, basenreichen Lehm- und Tonböden vor. Der Standort kann mäßig saver bis alkalisch sein. Der Höhenzuwachs der Linde kulminiert im Alter von 10 bis 20 Jahren. Die Winterlinde wächst optimal bei gemäßigten Temperaturen, bei mittleren Jahrestemperaturen von $8-9^{\circ} \mathrm{C}$. Milde, sommerwarme, vor kalten Winden geschützte Lagen mit Niederschlägen von 1500 bis $1800 \mathrm{~mm}$ sind für das Wachstum der Winterlinde ideal. Die Winterlinde wächst in ozeanisch und kontinental geprägten Gebieten, sie erträgt aber auch lufttrockene Lagen. Die Winterlinde gilt als winterhart. 
Ein biologischer Vorzug der Linde ist ihre bekannte Eignung als Nahrungsquelle für Bienen. Des Weiteren ist ihre leicht zersetzbare, stickstoffreiche Streu vorteilhaft für den Nährstoffkreislauf (Leibundgut, 1953). Eine Schwierigkeit bei der waldbaulichen Verwendung der Linde ist, dass sie vom Wild sehr gern verbissen und verfegt wird (Koss, 1982).

\subsection{Standortanforderungen}

Eine Grundvoraussetzung für die Baumartenwahl ist die ökologische Angepasstheit der Pflanzen an den Standort. Deshalb sollte jeder Pflanzung eine Standortanalyse bestehend aus Bodenanalyse, Klimaverhältnissen, Höhenlage und Exposition vorausgehen, aufgrund deren Ergebnisse passende Baumarten gewählt werden können. Eine standortangepasste Baumartenwahl beinhaltet eine optimale Ausnutzung des Biomassepotenzials und Dauerhaftigkeit des Standorts sowie eine erhöhte Widerstandsfähigkeit der Pflanzen gegenüber Krankheiten und Parasiten. Untersuchungen zum Biomassepotenzial verschiedener Baumarten auf landwirtschaftlichen Flächen sind aus der vorhandenen Literatur aber nur lückenhaft zu finden. Hierzu sind weiterführende Untersuchungen notwendig.

Weiden und Pappeln können laut Traupmann (2004) ab einer Bodenpunktzahl von 30 angebaut werden, wobei eine ausreichende Wasserversorgung besonders wichtig ist. Sandige, leichte Böden sind deshalb weniger geeignet für einen Anbau. Voraussetzung für hohe Biomasseerträge ist eine tiefgründige Durchwurzelbarkeit des Bodens (mind. $60 \mathrm{~cm}$ ). Pappeln können im Vergleich zu Weiden auch unter ungünstigeren Bedingungen (geringere Wasserversorgung, flachgründiger Boden) vergleichsweise höhere Erträge bilden. Unter Berücksichtigung ökonomischer Kriterien sollte ein Anbau auf Grenzstandorten jedoch nicht in Frage kommen. In

Tab 2.4 werden die in Anlehnung an Schlüter (1990) für häufig vorkommende Standorte geeigneten schnellwachsenden Laubgehölze aufgeführt. 
Tab. 2.4: Für häufig vorkommende Böden geeignete Baumarten (Schlüter, 1990)

\begin{tabular}{|c|c|c|}
\hline \multirow{4}{*}{$\begin{array}{c}\text { Feinerdehaltige steinige oder } \\
\text { kiesige Böden }\end{array}$} & Nährstoffarm, trocken & $\begin{array}{l}\text { Betula pendula } \\
\text { Populus spec. } \\
\text { Salix spec. }\end{array}$ \\
\hline & Nährstoffarm, frisch bis feucht & $\begin{array}{l}\text { Betula pendula } \\
\text { Populus spec. } \\
\text { Salix spec. } \\
\text { Sorbus aucuparia }\end{array}$ \\
\hline & Nährstoffreich, trocken & $\begin{array}{l}\text { Betula pendula } \\
\text { Populus spec. } \\
\text { Salix spec. } \\
\text { Sorbus aucuparia }\end{array}$ \\
\hline & Nährstoffreich, frisch bis feucht & $\begin{array}{c}\text { Acer pseudoplatanus } \\
\text { Alnus glutinosa } \\
\text { Alnus incana } \\
\text { Betula pendula } \\
\text { Populus tremula } \\
\text { Salix spec. }\end{array}$ \\
\hline \multirow[b]{2}{*}{ Sandige Böden } & Nährstoffarm, trocken & $\begin{array}{l}\text { Betula pendula } \\
\text { Populus spec. } \\
\text { Sorbus aucuparia }\end{array}$ \\
\hline & Nährstoffarm, frisch bis feucht & $\begin{array}{l}\text { Betula pendula } \\
\text { Betula pubescens } \\
\text { Populus spec. } \\
\text { Salix spec. } \\
\text { Sorbus aucuparia }\end{array}$ \\
\hline \multirow{4}{*}{ Lehmige Böden } & (Mäßig) nährstoffarm, trocken & $\begin{array}{l}\text { Betula pendula } \\
\text { Populus spec. } \\
\text { Sorbus aucuparia }\end{array}$ \\
\hline & $\begin{array}{c}\text { (Mäßig) nährstoffarm, frisch bis } \\
\text { feucht }\end{array}$ & $\begin{array}{l}\text { Betula pendula } \\
\text { Populus spec. } \\
\text { Salix spec. } \\
\text { Sorbus aucuparia }\end{array}$ \\
\hline & Nährstoffreich, trocken & $\begin{array}{l}\text { Betula pendula } \\
\text { Populus spec. }\end{array}$ \\
\hline & Nährstoffreich, frisch bis feucht & $\begin{array}{c}\text { Acer pseudoplatanus } \\
\text { Alnus glutinosa } \\
\text { Alnus incana } \\
\text { Betula pendula } \\
\text { Populus spec. } \\
\text { Salix spec. } \\
\text { Tilia cordata }\end{array}$ \\
\hline \multirow[b]{2}{*}{ Tonige Böden } & $\begin{array}{c}\text { (Mäßig) nährstoffarm, } \\
\text { wechseltrocken bis wechselfeucht }\end{array}$ & $\begin{array}{l}\text { Sorbus aucuparia } \\
\text { Tilia cordata }\end{array}$ \\
\hline & $\begin{array}{c}\text { Nährstoffreich, wechseltrocken } \\
\text { bis wechselfeucht }\end{array}$ & $\begin{array}{c}\text { Acer pseudoplatanus } \\
\text { Alnus glutinosa } \\
\text { Alnus incana } \\
\text { Salix spec. } \\
\text { Sorbus aucuparia } \\
\text { Tilia cordata }\end{array}$ \\
\hline
\end{tabular}


Durch eine standortangepasste Baumartenwahl kann auf eine Düngung der Fläche verzichtet werden, insbesondere da die Ansprüche an die Nährstoffversorgung in der Landwirtschaft höher ausfallen als in der Forstwirtschaft (Pallast et al., 2006). Waldstandorte sind in der Regel vergleichsweise nährstoffarm, und die Ackerflächen bringen zum Zeitpunkt der Pflanzung einen großen Vorrat an Nährstoffen mit. So sind nach forstlicher Einteilung Ackerstandorte in der Regel als gut mesotroph bis eutroph anzusprechen (Hofmann, 1998), was sich mit den Anforderungen der Forstwirtschaft für den Pappelanbau an den Boden deckt (Schultzke et al., 1990).

Nach Einschätzung von Hofmann (1998) erübrigt sich beim Anbau von Energiehölzern eine Düngung der Fläche für mindestens 15 Jahre, da zunächst die großen Nährstoffpotenziale ehemaliger Ackerflächen langsam abgeschöpft werden können. Auch Jug et al. (1999) kommen im Rahmen von standortkundlichen Untersuchungen zu dem Ergebnis, dass die untersuchten Balsampappeln und Aspen auf den betrachteten ehemaligen Ackerstandorten mit allen Nährstoffen gut versorgt sind. Ihren Untersuchungen zufolge ist durch zusätzliche Düngung keine signifikante Wuchsverbesserung in den ersten Rotationszyklen nach der Aufforstung zu erreichen.

Der durch Mineralisation pflanzenverfügbare Stickstoff gewährleistet im Boden für mindestens 10-15 Jahre ein optimales Wachstum von Balsampappeln und Aspen (Jug et al., 1999). Dies gilt ebenso für Phosphor, Kalium und Magnesium, deren Nährstoffkonzentration in den Blättern über die ersten zehn Jahre annähernd konstant war oder sogar anstieg. Der Nährstoffbedarf von Salix viminalis unterscheidet sich allerdings von den beiden anderen Arten. Um die Erträge zu maximieren, sind hier regelmäßige Stickstoffdüngungen durchzưühren (Hofmann-Schielle et al., 1999). Während die Versorgung mit Phosphor, Kalium, Calcium und Magnesium auf allen untersuchten Standorten ausreichend gesichert war, hat die Weide eine sehr hohe Stickstoffbedürftigkeit und reagiert deshalb als einzige Art auf eine erhöhte N-Zufuhr (Jug et al., 1999). 


\subsection{Erntezyklen}

\subsubsection{Mini-Rotation}

Bei Mini-Rotationen erfolgt die Ernte der Bäume bereits nach zwei- bis dreijähriger Wachstumszeit. Um nach dieser kurzen Entwicklungszeit wirtschaftliche Erträge zu erzielen, sind sehr dichte Bestände (16.000-20.000 Bäume pro Hektar) zu etablieren. Diese Nutzung führt zu hohen Masseleistungen je Hektar in Form von sehr schwachem Holzmaterial (3-4 cm Stammdurchmesser), das ausschließlich für Heizzwecke eingesetzt wird. Meist werden Weiden in Mini-Rotation genutzt.

Tab. 2.5: Wichtige Weidensorten für den Kurzumtrieb (Röhricht \& Ruscher, 2004)

\begin{tabular}{|c|c|c|}
\hline Weidensorte & Kreuzung & Bemerkung \\
\hline $\begin{array}{l}\text { Zieverich } \\
\text { Carmen } \\
\text { Ingeborg } \\
\text { Ulf } \\
\text { Rapp } \\
\text { Orm } \\
\text { Loden }\end{array}$ & S. viminalis & $\begin{array}{l}\text { Mittlere bis hohe Ertragsleistung in Mini- } \\
\text { Rotation, mittlere bis gute Resistenz gegen } \\
\text { Blattrost }\end{array}$ \\
\hline Jorr & $\begin{array}{l}\text { S. viminalis } \\
\text { (Kreuzung niederländischer Klone) }\end{array}$ & $\begin{array}{l}\text { Hohes Ertragspotenzial, zügige } \\
\text { Jugendentwicklung, mittlere Resistenz gegen } \\
\text { Blattrost }\end{array}$ \\
\hline Tora & $\begin{array}{l}\text { S. schwerinii } x \text { S. viminalis } \\
\text { (Kreuzung sibirische Korbweide } \mathrm{x} \\
\text { Orm) }\end{array}$ & $\begin{array}{l}\text { Hohe Zuwachsraten, weitgehende } \\
\text { Blattrostresistenz, geringer Befall mit } \\
\text { Gallmücken, kaum Wildverbiss }\end{array}$ \\
\hline Torhild & $\begin{array}{l}\text { (S. viminalis } \times \text { S. schwerinii) } \times \text { S. } \\
\text { viminalis }\end{array}$ & $\begin{array}{l}\text { Sehr hohes Ertragsniveau, weitgehend } \\
\text { resistent gegen Blattrost }\end{array}$ \\
\hline Sven & $\begin{array}{l}\text { S. viminalis } \times \text { (S. viminalis } \times \text { S. } \\
\text { schwerinii) } \\
\text { (Kreuzung der Sorten Jorum und } \\
\text { Biörn) }\end{array}$ & $\begin{array}{l}\text { Hochertragssorte, hohe Resistenz gegen } \\
\text { Blattrost }\end{array}$ \\
\hline Olof & $\begin{array}{l}\text { S. viminalis } \times \text { (S. viminalis } \times S \text {. } \\
\text { schwerinii) } \\
\text { (Kreuzung der englischen Sorte } \\
\text { Bowles Hybrid mit Biörn) }\end{array}$ & $\begin{array}{l}\text { Kleinwüchsig, hohe Triebzahl, hohes } \\
\text { Ertragsniveau, kein Blattrostbefall }\end{array}$ \\
\hline Gudrun & S. dascylados & $\begin{array}{l}\text { Hohe Frosttoleranz, ausgeprägte Resistenz } \\
\text { gegen Blattbockkäfer, geringer Wassergehalt } \\
\text { zur Ernte, gute Unkrautunterdrückung, } \\
\text { teilweise Wildschäden }\end{array}$ \\
\hline Tordis & $\begin{array}{l}\text { (S. viminalis } \times \text { S. schwerinii) } \times \text { S. } \\
\text { viminalis } \\
\text { (Kreuzung der Sorten Tora und Ulv) }\end{array}$ & Blattrostresistenz, hohes Ertragspotenzial \\
\hline
\end{tabular}


Schwarze und Röhricht (2006) haben Untersuchungen zur Ertragsleistung verschiedener Pappel- und Weidensorten am Standort Kalkreuth (Landkreis RiesaGroßenhain) durchgeführt, der einen stark sandigen Lehm mit der Ackerzahl 49 darstellt. Für die zwei- und dreijährigen Umtriebszeiten wurden hohe Bestandsdichten (17.778 Bäume/ha) gewählt. Zur Pflanzung sind Steckruten von $100-125 \mathrm{~cm}$ Länge verwendet worden. Betrachtet man die jährlichen Erträge an Trockenmasse (TM) über die bisher erfolgten vier Umtriebszeiten, zeichnen sich zwischen den Baumarten und Sorten deutliche Unterschiede ab. Aus dem Sortenvergleich sind die Korbweidensorte "Zieverich" (14 †TM/ha) und die Balsampappelsorten "Beaupré" (13,9 †TM/ha) sowie "Max 1" (12,6 + TM/ha) mit dem höchsten durchschnittlichen Gesamtzuwachs pro Hektar und Jahr im Mittel der vier Umtriebe hervorzuheben. Auch die Sorten "Muhle Larsen" und "Max 3" sind mit jährlichen Ertragsleistungen von 10,8 † TM/ha bzw. 11,7 † TM/ha für den Anbau als schnell wachsend zu betrachten.

Die von den Sorten "Astria" und "Münden" erreichte unterdurchschnittliche Ertragsleistung unterstreicht die Feststellung, dass Aspen für die Mini-Rotationsnutzung weniger geeignet sind. Der optimale Ernterhythmus von Aspen liegt bei etwa 15 Jahren im Bereich der Maxi-Rotation (Liesebach et al., 1999). Im zweijährigen Ernteturnus schneiden die Balsampappeln "Beaupré" und "Max 3" am besten ab. Die Korbweide "Zieverich" entwickelt nach den vorliegenden Ergebnissen erst im dreijährigen Umtrieb einen starken jährlichen Biomassezuwachs. Insgesamt wurde über die vier Umtriebszeiten eine stabile Produktion an Holzbiomasse nachgewiesen. Aus erntetechnischer Sicht ist hervorzuheben, dass bei zweijährigem Umtrieb durchschnittliche Stammdurchmesser von $29 \mathrm{~mm}$ (20 mm bei Aspen; 36-38 mm bei "Max 3" und "Beaupré") erreicht wurden. Im dreijährigen Umtrieb entwickeln die Bäume Stämme von 35 mm (27 mm "Zieverich"; 47 mm "Max 1“) Durchmesser. Dabei ist die Anzahl der Nebentriebe von über $2 \mathrm{~m}$ Länge bei der Weide mit 9,7 bis 13 Trieben deutlich größer als bei den Pappelsorten (0,6-4,4 Triebe). Die Wuchshöhe der Bäume liegt zwischen 6 und $8 \mathrm{~m}$. 


\subsubsection{Midi-Rotation}

Die Ernte der Bäume erfolgt alle 4 bis 6 (max. 10) Jahre. In dieser Wachstumszeit erzielen die Bäume stärkere Stammdurchmesser $(6-8 \mathrm{~cm})$ und höhere Stammeinzelgewichte als bei zwei- bis dreijährigem Ernterhythmus. Midi-Rotationen erfordern deshalb geringere Bestandszahlen zum Ertragsaufbau. Günstig sind Baumzahlen von 6.000 - 9.000 Stück/ha.

Laut einer von Traupmann (2004) erwähnten Studie (FBVA 1997) produzierten fünfjährige Balsampappelaufwüchse im Verband $0,4 \mathrm{~m} \times 2,5 \mathrm{~m}$ pro Flächeneinheit ungefähr die gleiche Menge an Biomasse wie zehnjährige Aufwüchse in einem Verband 2,5 m x $2 \mathrm{~m}$. So lieferten Dünnholzproduktionen in Umtriebszeiten zwischen 6 und 10 Jahren bei einem etwas weiteren Verband von 2.000 bis 7.000 Stück pro Hektar ebenfalls hohe Erträge wie Mini-Rotationen. Wegen der zusätzlich eingesparten Pflanz- und Erntekosten ist diese Bewirtschaftungsform wirtschaftlicher als eine Produktion in kurzen bzw. extrem kurzen Umtriebszeiten (Traupmann, 2004).

Tab. 2.6: Wichtige Pappelsorten für den Kurzumtrieb (Röhricht \& Ruscher 2004)

\begin{tabular}{|c|c|c|}
\hline Kreuzungen und Sorten & Kreuzungspartner & Bemerkung \\
\hline $\begin{array}{l}\text { Muhle Larsen } \\
\text { Weser 1-6 } \\
\text { Scott-Pauley } \\
\text { Fritzi-Pauley } \\
\text { Columbia River } \\
\text { Trichobel }\end{array}$ & P. trichocarpa $\times$ P. trichocarpa & $\begin{array}{l}\text { Mittlere bis hohe Leistung bei } \\
\text { Midi-Rotation }\end{array}$ \\
\hline $\begin{array}{l}\text { Androscoggin } \\
\text { Hybrid 275/NE } 42\end{array}$ & $\begin{array}{l}\text { P. maximowiczii } \times P \text {. } \\
\text { trichocarpa }\end{array}$ & $\begin{array}{l}\text { Mittlere bis hohe Leistung bei } \\
\text { Midi-Rotation }\end{array}$ \\
\hline $\begin{array}{l}\text { Max 1-5 } \\
\text { Rochester }\end{array}$ & P. nigra $\times$ P. maximowiczii & $\begin{array}{l}\text { Hohe Ertragsleistung bei Mini- } \\
\text { und Midi-Rotation }\end{array}$ \\
\hline $\begin{array}{l}\text { Raspalje } \\
\text { Beaupré } \\
\text { Rap } \\
\text { Unal } \\
\text { Barn } \\
\text { Donk } \\
\text { Boelare }\end{array}$ & P. deltoides $\times$ P. trichocarpa & $\begin{array}{l}\text { Sehr hohe Ertragsleistung bei } \\
\text { allen Rotationstypen }\end{array}$ \\
\hline $\begin{array}{l}\text { Ahle 1-20 } \\
\text { Tapiau 1-8 }\end{array}$ & P. tremula $\times$ P. tremula & \\
\hline $\begin{array}{l}\text { Astria } \\
\text { Münden 1-20 }\end{array}$ & P. tremula $\times P$. tremuloides & Hohe Leistung bei Maxi-Rotation \\
\hline
\end{tabular}




\subsubsection{Maxi-Rotation}

Bei der Maxi-Rotation werden die Bestände 10 bis 20 Jahren stehen gelassen. Der Ertrag wird in noch stärkerem Maße über das Einzelgewicht realisiert. Die Stammdurchmesser betragen $10-12 \mathrm{~cm}$. Baumzahlen von etwa 1500 - 3000 Stück/ha gewährleisten bei dieser Nutzungsrichtung einen optimalen Aufwuchs. Inwieweit diese Angaben für die Baumarten im Einzelnen zutreffen, ist noch in langfristigen Anbauversuchen zu klären.

Für die Maxi-Rotation eignen sich die eher alternativen Baumarten wie Aspe sowie Bergahorn, Winterlinde, Eberesche und Erle, die zwar relativ schnellwüchsig sind, aber mit den Hochleistungsklonen der Pappel und den Weidenzüchtungen in Bezug auf die Wuchsleistungen in sehr kurzen Umtriebszeiten nicht mithalten können. Die Biomasseleistung der oben genannten Baumarten ist trotzdem in längeren Umtriebszeiten nicht zu unterschätzen. Bei den von Liesebach et al. (1999) erwarteten Wuchsentwicklungen der Aspe in Maxi-Rotation wird deren Holz nicht nur für die energetische Nutzung in Frage kommen, sondern vielmehr in der Papier- und Zellstoffindustrie Absatz finden. So zeigten deren Versuchsergebnisse, dass auch auf nährstoffarmen Flächen mit nur durchschnittlicher Wasserverfügbarkeit von Hybridaspen durchschnittlich 100 t/ha Biomasse innerhalb von zehn Jahren produziert werden können. Um einen maximalen durchschnittlichen Biomassezuwachs zu erreichen, werden Umtriebszeiten von mehr als zehn Jahren empfohlen (Liesebach et al., 1999). Pflanzenzahlen von 5555 Stück $(2,0 \mathrm{~m} \times 0,9 \mathrm{~m})$ und 4200 Stück $(2,0 \mathrm{~m} \times 1,2 \mathrm{~m})$ erwiesen sich als geeignet.

Längere Umtriebszeiten können ökologische Vorteile für die Umwelt bedeuten. So wirken Energieholzplantagen aufgrund der mehriährigen Umtriebszeit bodenverbessernd. Untersuchungen belegen signifikant erhöhte Gehalte an organischem Kohlenstoff $\left(C_{\text {org }}\right)$ der Oberböden als Folge von Laub- und Wurzelstreumassen und fehlender Bodenbearbeitung (Kahle \& Boelcke, 2004). Vor allem die mehriährige Umtriebszeit wirkt sich positiv auf das Bodengefüge aus. Resultierend aus der Anreicherung von organischer Substanz zeigten die mit Pappeln 
und Weiden bestandenen Parzellen zudem signifikant verringerte Rohdichten und erhöhte Porosität sowie eine damit verbundene Verbesserung der Wasserretention. Der Anteil der Mittelporen, die das pflanzenverfügbare Wasser führen, erhöhte sich seit Versuchsbeginn signifikant (Kahle \& Boelcke, 2004).

\subsection{Flöchenvorbereitung}

Die Anlage von Kurzumtriebsplantagen auf Ackerflächen ist in der Regel mit einer flächenvorbereitenden Bodenbearbeitung verbunden, meist dem Vollumbruch auf 30 $\mathrm{cm}$. Dabei werden in der Literatur sowohl das Pflügen der Fläche im Herbst als auch im Frühjahr erwähnt (Burger, 2004; Diederichs, 1990; Dimitri, 1988; Hofmann, 2004; Traupmann, 2004; Röhricht, 2005).

Aus Gründen des Boden- und Gewässerschutzes sind intensive Bodenvorbereitungen allerdings sehr kritisch zu sehen. Es sind erhöhte Auswaschungsverluste insbesondere von Nitraten möglich, da zum einen der Boden durch die tiefgründige Bearbeitung höhere Mineralisationsraten aufweist und zum anderen jeglicher Pflanzenbewuchs über Winter fehlt, welcher der Nitratverlagerung durch eine Stickstoff-Festlegung in der pflanzlichen Biomasse entgegenwirken könnte (Meyer-Marquart et al., 2006). Weiterhin ist auf erosionsgefährdeten Standorten mit vermehrtem Oberflächenabfluss und erhöhter Bodenerosion zu rechnen, wenn der Boden über Winter ohne Vegetationsbestand oder Mulchschicht den Witterungsbedingungen ausgesetzt ist (Meyer-Marquart et al., 2006). Wegen des Boden- und Gewässerschutzes sollte bei der Anlage von Kurzumtriebsplantagen auf das Pflügen der Fläche im Herbst möglichst verzichtet werden. Auf leichten Böden ist dies nicht zwingend erforderlich, sondern kann auf das Frühjahr begrenzł werden (Hofmann, 2004). Die Bodenbearbeitung im Frühjahr sollte in dem Fall mit möglichst geringer Intensität erfolgen. Durch konservierende Bodenbearbeitungs- und Bestellverfahren im Frühjahr wird ein Mineralisationsimpuls verhindert, der zu erhöhten Nitratauswaschungen im Frühjahr oder Herbst des Setz- bzw. Pflanzjahres beitragen kann. Außerdem reduziert ein im 
Frühjahr durchgeführtes Mulchverfahren die Auswaschungs- und Erosionsgefährdung im Winter, und auch in der Phase der Bestandsetablierung wird der noch unbedeckte Boden sicher vor Wind- und Wassererosion geschützt (Meyer-Marquart et al., 2006).

\subsection{Begleitwuchsregulierung}

Da landwirtschaftliche Flächen enorme Diasporenbänke bevorraten, sind wirksame Maßnahmen der Begleitwuchsregulierung zu ergreifen, um eine Kultur erfolgreich etablieren zu können. In welcher Form die Unkrautbekämpfung durchzuführen ist, hängt stark von der Flächenvornutzung und dem verwendeten Pflanzenmaterial ab. In der Phase der Bestandsetablierung wird der Unkrautdruck bei vielen Versuchsanlagen durch den Einsatz von Herbiziden reguliert. Wie beim Ackerbau gilt aber, dass vorrangig Nachauflaufpräparate und nur bedingt Vorauflaufmittel angewendet werden sollten, um potenzielle Wirkstoffausträge mit dem Sickerwasser, dem Dränwasser und insbesondere mit dem Oberflächenabfluss zu vermeiden (Meyer-Marquart et al., 2006).

Das Verfahren der mechanischen Unkrautregulierung führt zu einer oberirdischen Reduzierung des Unkrautdrucks, so dass der Aufwuchs der Setzlinge nicht oder im geringerem Umfang beeinträchtigt wird. Zusätzlich wird der Nährstoffgehalt des Bodens durch Schichten aus frisch geschnittenem Gras merklich beeinflusst. Nach Fang et al. (2008) weisen die mit Gras gemulchten Flächen eine höhere Konzentration an absolutem $\mathrm{N}$ sowie verfügbaren $\mathrm{N}, \mathrm{P}$ und $\mathrm{K}$ auf als die nicht gemulchten Kontrollflächen. Infolge des Mulchverfahrens wurden das Höhenwachstum und der Brusthöhendurchmesser der untersuchten Pappelplantage signifikant erhöht (Fang et al., 2008). Bei der Flächenanlage der Kurzumtriebsplantage ist bei mechanischer Unkrautregulierung zwischen den Pflanzreihen der Pflanzverband auf die Mäherbreite abzustimmen. 
Aus Österreich berichtet Traupmann (2004) von positiven Erfahrungen mit Untersaaten. Demnach können Untersaaten, die vor der Pflanzung der Stecklinge eingesät werden, den Begleitwuchs verhindern und die Kulturpflegemaßnahmen erheblich reduzieren. Versuche mit Getreide wie Wintergerste und Hafer als Untersaat ergaben eine hauptsächliche Unterdrückung des Begleitwuchses bei nur geringer Beeinträchtigung der Kurzumtriebspflanzen. Auch Leguminosen können nach Traupmann (2004) als Untersaat verwendet werden. Boelcke (2006) stellte in ihren Untersuchungsergebnissen dar, dass Untersaaten zur Unkrautunterdrückung und Bodenbedeckung nicht empfohlen werden können, da sie für Baumsprösslinge eine starke Konkurrenz darstellen. Auch Jylhä et al. (2006) räumten die Möglichkeit ein, dass sich die Konkurrenz um Ressourcen zwischen eingesätem Weißklee und Baumsämlingen schwerwiegender auf die Ausfallhäufigkeit und das Baumvolumen auswirkt als die Konkurrenz zwischen der natürlichen Vegetation und den Sämlingen.

Versuche mit Untersaaten wurden auch von Scholz et al. (2004) auf einem sandigen Standort nordöstlich von Potsdam (Versuchsfläche Bornim) mit sehr geringen Niederschlagssummen (durchschnittlich $477 \mathrm{~mm} / \mathrm{a}$ ) durchgeführt. Neben Baumart, Untersaat und Düngung wurde auch das Rotationsintervall (Umtriebszeit) variiert. Die gemessenen Erträge wiesen eine außerordentliche Spanne auf und wurden weniger von der Düngergabe als vielmehr von Untersaat und Alter des Bestands bestimmt. Die verwendete Grasuntersaat erwies sich als erheblicher Wasser- und Nährstoffkonkurrent, der bei dem leistungsfähigen Balsampappelhybrid "Japan 105" in den ersten vier Jahren je nach Düngeregime und Rotationsintervall eine Ertragseinbuße von 10 bis $65 \%$ bewirkt hat. Im Gegensatz zur Weide wurde durch das großblättrige Laub der Pappeln die Untersaat - und auch das Unkraut - unterdrückt, so dass sich die Ertragsdifferenz im Laufe der Zeit verringerte. Die von Scholz et al. (2004) festgestellten Ertragsverluste durch Untersaaten in den ersten Jahren sind vor dem Hintergrund der besonderen Standortbedingungen zu bewerten; der sandige Versuchsstandort mit den sehr geringen Jahresniederschlägen machte jede Wasserkonkurrenz problematisch und lässt somit eine Übertragung der Ergebnisse auf 
wüchsigere Standorte mit besserer Wasserversorgung nicht zu (Meyer-Marquart et al., 2006).

\subsection{Bodenhilfsstoffe}

\subsubsection{Mykorrhiza}

Um die Startbedingungen und des Kulturerfolges der neu gepflanzten Bäume zu verbessern, können Bodenhilfsstoffe wie Mykorrhiza und Hydrogel angewendet werden. Da infolge der langiährigen Nutzung als Acker bzw. Grünland keine BaumMykorrhiza, auf welche die Waldbäume angewiesen sind, mehr im Boden zu erwarten sind, könnte eine Zugabe der geeigneten Mykorrhizapilze den Pflanzen eine bessere Wasser- und Nährstoffversorgung gewährleisten.

Ektomykorrhiza-Pilzen beeinflussen die Ernährung und Entwicklung der Waldbäume auf verschiedene Weisen und beteiligen sich an mehreren physiologischen Schlüsselprozessen bei im Feinwurzelbereich (Blaschke, 1980). Ekłomykorrhizen können auf Grund ihrer Morphologie ein viel größeres Bodenvolumen erschließen als nicht mykorrhizierte Feinwurzeln, was für die mykorrhizierten Waldbäume ein Vorteil im Hinblick auf die mineralische Ernährung und die Wasserversorgung bedeutet (Blaschke, 1980). Zudem führt die Mikroflora, die mit der Ektomykorrhiza im Feinwurzelbereich der Waldbäume vergesellschaftet ist, zu einer höheren biotischen Aktivität in der Rhizosphäre (Blaschke, 1980).

\subsubsection{Hydrogel}

Neben Mykorrhiza-Pilzen sind Bodenbedingungen und Wasserhaushalt Schlüsselelemente bei der Aufforstung. Mithilfe von Hydrogel könnten die Sämlinge in der kritischen Anwuchsphase durch eine verbesserte Wasserversorgung unterstütz† werden. Wasserspeicherne Substanzen für dir Pflanzenanzucht wurden in den 1950er 
Jahren entwickelt. Hydrogele sind hochvernetzte Polyacrylamide, welche in der Lage sind, das 200-fache ihres eigenen Gewichtes an Wasser zu absorbieren und speichern und so den Wasserstress für die Pflanzen zu reduzieren.

Der Effekt von Hydrogelen auf die Überlebensrate und das Wachstum von Gehölzen fiel in vergangenen Untersuchungen unterschiedlich aus. Zum einen verbesserten Hydrogele die Wasserverfügbarkeit für die Pflanzen (Martyn \& Szot, 2001; Akhter et al., 2004) und erhöhten die Überlebensrate und das Wachstum von Forstpflanzen auf trockenen Standorten (Hüttermann et al., 1999; Wu et al., 2005) und anderen suboptimalen Pflanzbedingungen (Viero \& Little, 2006). Die jungen Gehölze überlebten mit Hydrogel längere Trockenperioden als in den Kontrollböden (Savé et al., 1995; Al-Humaid \& Moftah, 2007). In anderen Untersuchungen hielt das Hydrogel die Feuchtigkeit eher zurück, als dass es den Pflanzen zur Verfügung stand (Tripepi et al., 1991) und konnte das Wachstum der Pflanzen nicht verbessern (Tsakaldimi, 1998). Ursachen hierfür kann laut Visnjic (2006) eine Überdosierung von Hydrogel sein, der in seinen Untersuchungen eine Dosierung von $5 \mathrm{~g} / \mathrm{dm}^{3}$ empfiehlt. Bei einer Hydrogelkonzentration von $10 \mathrm{~g} / \mathrm{dm}^{3}$ erstickten die Pflanzen an der erhöhten Wassermenge im Wurzelraum. 


\section{Material und Methoden}

\subsection{Versuchsfläche Sudheide (Landkreis Gütersloh)}

\subsubsection{Standortsbeschreibung}

Gütersloh liegt in der Emssandebene südwestlich des Teutoburger Waldes und damit am nordöstlichen Rand der Westfälischen Bucht. Gütersloh gehört der gemäßigten Klimazone Mitteleuropas an. Im langiährigen Mittel von 1961-1990 beträgt die Jahrestemperatur in Gütersloh $9,2^{\circ} \mathrm{C}$ und es fallen $762 \mathrm{~mm}$ Niederschlag. Damit ist das Klima in Gütersloh wärmer und feuchter, als im deutschen Mittel $\left(8,4^{\circ} \mathrm{C} / 700 \mathrm{~mm}\right)$. Der wärmste Monat ist der Juli mit einer Durchschnittstemperatur von $18,3^{\circ} \mathrm{C}$, der kälteste Monat der Januar mit $2,4^{\circ} \mathrm{C}$. Die Vegetationszeit davert durchschnittlich 170 Tage im Jahr und weist eine mittlere Temperatur von $15,5^{\circ} \mathrm{C}$ auf. (Quelle: DWD)

Im Untersuchungsgebiet herrschen saure, nährstoffarme Podsol-Böden vor, die aus Ablagerungen während des

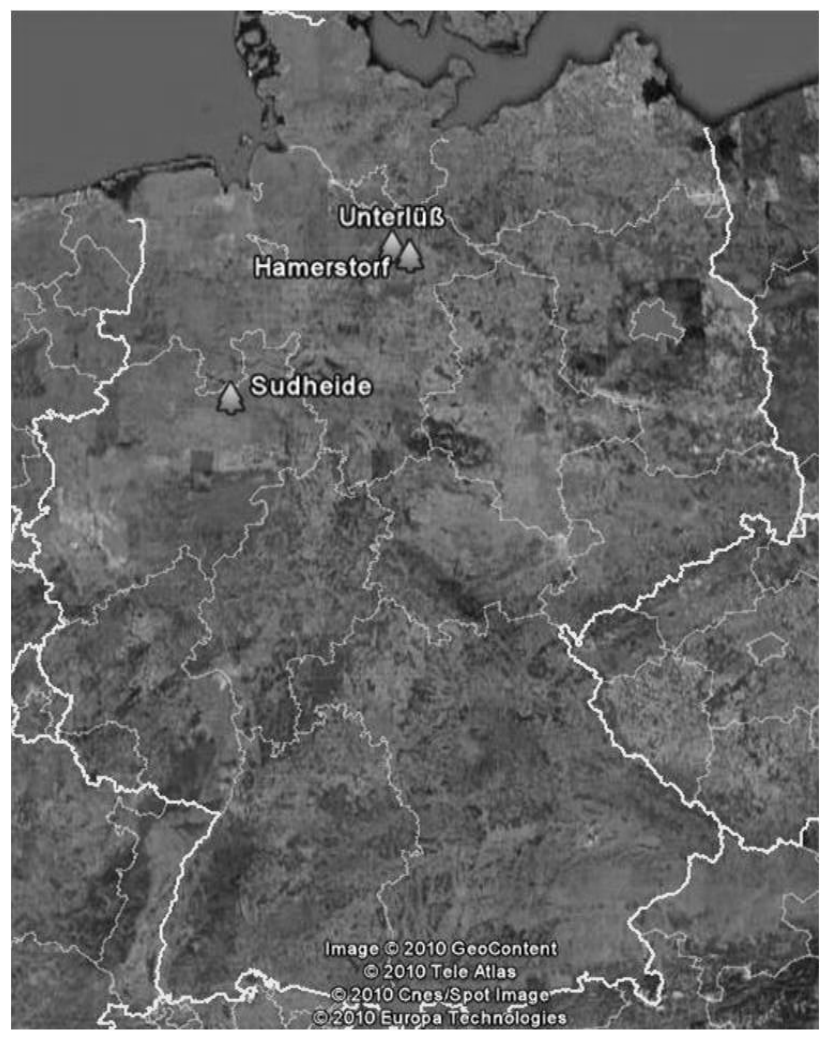

Quartärs einerseits aus glazialem Schmelzwasser und andererseits durch FlussSedimente hervorgegangen sind. Stellenweise sind die Ablagerungen von Flugsanden überdeckt und bilden dann Podsol-Regosol-Böden. Insgesamt ist das Unterschungsgebiet also von einem unfruchtbaren, fein- bis mittelsandigen Boden geprägt. Die Bodenpunkte liegen zwischen 25 und 30. 
Die auf der Versuchsfläche vor der Kulturbegründung genommenen Bodenhorizonte zeigten einen 20 bis $30 \mathrm{~cm}$ tiefen mineralischen Oberboden, der durch eine regelmäßige landwirtschaftliche Bearbeitung geprägt wurde. Daran schloss sich ein durch Verwitterung verbraunter mineralischer Unterboden an. Die $N_{\min }$-Analysen, die von Lamersdorf (2007) durchgeführt wurden, spiegelten die deutliche Belastung dieses Standorts mit vor allem Nitratstickstoff wieder, was über die Analyse von Nitrat in der Bodenlösung bestätigt wurde. Auf der Versuchsfläche Sudheide zeigte sich kein systematischer Zusammenhang zwischen der Art der Vornutzung und den ermittelten $\mathrm{N}_{\text {min }}$-Gehalten bzw. -Vorräten. Der Vorrat an $\mathrm{N}_{\text {min }}$ (nur $\mathrm{NO}_{3}$ und $\mathrm{NH}_{4}$ ) in $0-20 \mathrm{~cm}$ Bodentiefe wurde mit Werten von $>10$ bis $20 \mathrm{~kg} / \mathrm{ha}$ insgesamt als mittel bis hoch eingeschätzt. Von Lamersdorf (2007) wurde angenommen, dass sich größere Mengen an $\mathrm{N}_{\min }$ zusätzlich in tieferen Bodenschichten befinden, zumindest bis zum Erreichen der Pflugsohle in $30 \mathrm{~cm}$ Bodentiefe.

Auffallend war nach Lamersdorf (2008) die relativ geringe Dichte im Oberboden, welche vermutlich durch die intensive Bodenbearbeitung aus der vormaligen landwirtschaftlichen Nutzung verursacht wurde. Die C-Gesamt- bzw. Corg-Gehalte waren auffallend hoch. Diese stiegen im Unterboden auf bis über $4 \%$ an, was an der starken Dunkelfärbung des Bodenmaterials sichtbar wurde. Auch die Vorräte an austauschbarem Calcium waren erhöht, während die Vorräte an Kalium und Magnesium geringfügig reduziert waren.

\subsubsection{Flächenplan}

Die Versuchsfläche Sudheide $\left(51^{\circ} 52^{\prime} 35.00^{\prime \prime} \mathrm{N} ; 8^{\circ} 19^{\prime} 0.00^{\prime \prime} \mathrm{E}\right)$ unterteilte sich in eine Grünfläche und eine gepflügte und wiederverdichtete Ackerfläche und hat eine Größe von etwa 2 ha. Die Flächen wurden in Parzellen von 30 × 40 m unterteilt, die mit jeweils einer Baumart kultiviert wurden (Abb. 3.2). Um die gegenseitige Beeinflussung der Teilflächen zu minimieren, wurde zwischen den Parzellen ein Abstand von $2 \mathrm{~m}$ eingehalten 
Auf der Grünlandfläche wurden Aspe, die Pappelhybriden "Max 4" und "NE 42", die Weidensorte "Turbo", sowie Robinie gepflanzt. Auf der Ackerfläche wurden zusätzlich Bergahorn, Eberesche, Schwarzerle und Winterlinde gepflanzt. Durch die Einbindung von Baumarten wie Linde und Ahorn neben den bewährten schnellwachsenden Arten sollte die Frage geklärt werden, ob diese Baumarten mit einem erwarteten höheren ökologischen Wert messbare positive Effekte für Ökosystem und Landschaft bieten.

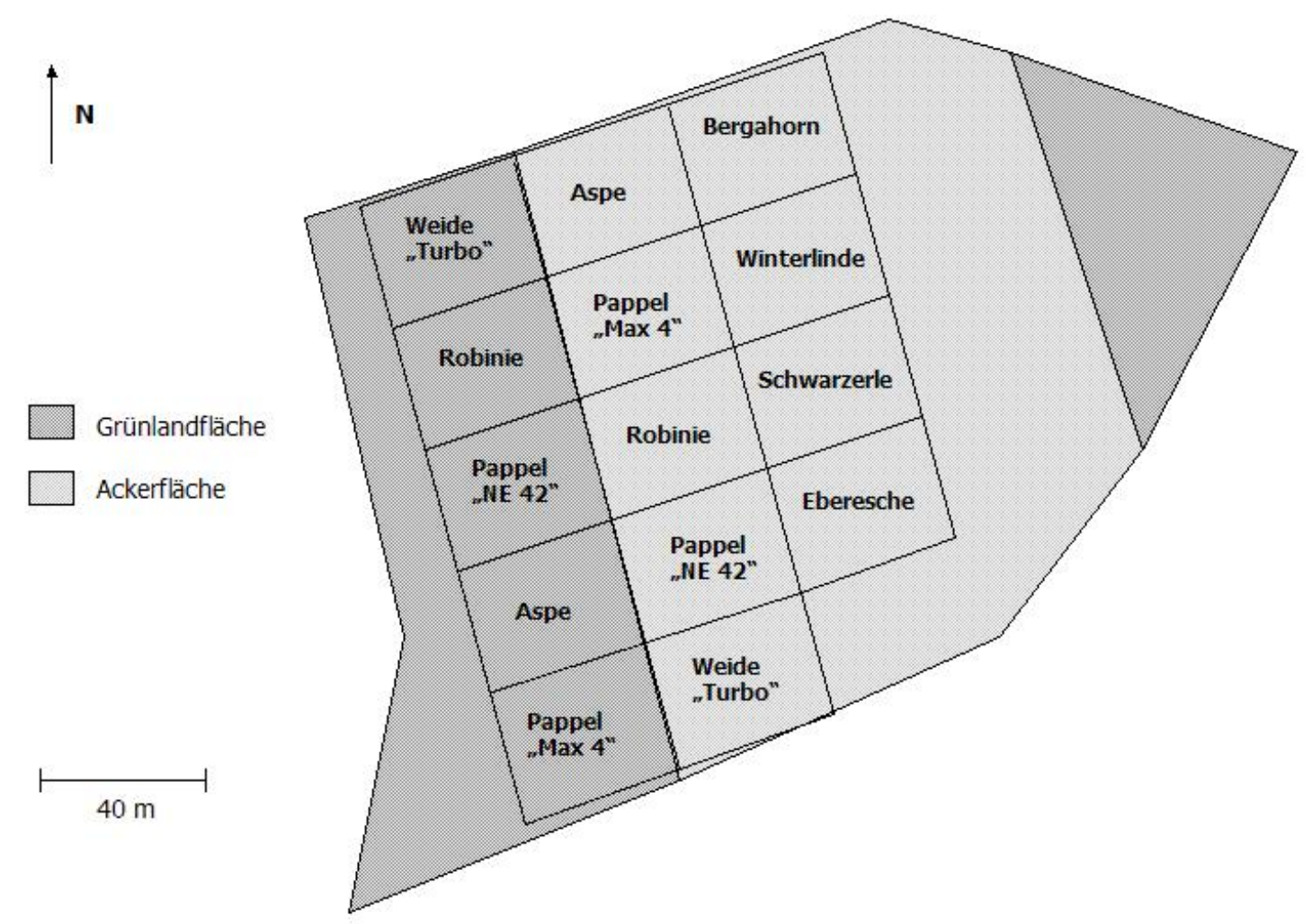

\section{Abb. 3.2: Schematischer Aufbau der Versuchsfläche Sudheide in Gütersloh}

Die Pflanzung von Weide und Pappel erfolgte mittels Stecklingen, bei den übrigen Baumarten wurden 2 bis 3-jährige Jungpflanzen verwendet (Tab. 3.1).

Die Kulturanlage erfolgte Mitte März 2007. Für die Pflanzung wurden die Pflanzlöcher mit einem Pflanzlochbohrer (20 cm Durchmesser) vorgebohrt. Die Bohrtiefe betrug etwa $19 \mathrm{~cm}$, so dass die Stecklinge noch etwa $1 \mathrm{~cm}$ aus dem Boden ragten. Aus Bohrtiefe und -durchmesser ergab sich ein Bodenaushub von 6 I. Die Stecklinge, die als Kontrolle dienten, wurden ohne Vorbohrung in den Boden gesteckt. 
Tab. 3.1: Auf der Fläche Sudheide verwendetes Pflanzenmaterial

\begin{tabular}{lll}
\hline Botanischer Name & Deutscher Name & Sortiment (Alter, Höhe) \\
\hline Populus tremula & Aspe (Zitterpappel) & 2j. $80 / 120$ \\
Acer pseudoplatanus & Bergahorn & 2j. $80 / 120$ \\
Sorbus aucuparia & Eberesche & 2j. $80 / 120$ \\
P. nigra x P. maximowiczii & Pappel "Max 4" & $20 \mathrm{~cm} \mathrm{Steckholz}$ \\
P. maximowiczii x P. trichocarpa & Pappel "NE 42" & $20 \mathrm{~cm} \mathrm{Steckholz}$ \\
Robinia pseudoacacia & Robinie & 2j. $80 / 120$ \\
Alnus glutinosa & Schwarzerle & 2j. $80 / 120$ \\
Salix viminalis & Weide "Turbo" & $20 \mathrm{~cm} \mathrm{Steckholz}$ \\
Tilia cordata & Winterlinde & 3j. $80 / 120$ \\
\hline
\end{tabular}

Das Pflanzschema orientierte sich in Anlehnung an das so genannte Nelder-Design (Abb. 3.3). Dabei variierten die Standräume für eine Baumart stufenlos, um nach mehreren Standjahren differenzierte Aussagen über die sich verstärkende intraspezifische Konkurrenzsituation und die Auswirkung auf Wuchsentwicklung und ökologische Effekte machen zu können. In den ersten beiden Vegetationsjahren, die in der vorliegenden Arbeit untersucht wurden, spielte diese intraspezifische Konkurrenz allerdings keine Rolle und wird in dem Ergebnisteil nicht bearbeitet.

Der Abstand zwischen den Pflanzreihen betrug 2,5 m. Die Abstände zwischen Pflanzen innerhalb einer Reihe reichten in Stufen von $1-3 \mathrm{~m}$. Aus den variierenden Pflanzabständen ergaben sich unterschiedliche Standräume je Pflanze und somit auch unterschiedliche Pflanzenzahlen je ha (Tab. 3.2).

Tab. 3.2: Standraumgrößen und Pflanzenzahlen/ha auf der Fläche Sudheide (Gütersloh)

\begin{tabular}{cccc}
\hline $\begin{array}{c}\text { Pflanzenabstand } \mathbf{x} \\
\text { Reihenabstand }\end{array}$ & Pflanzen/Parzelle & Standraumgröße & Pflanzenzahl/ha \\
\hline $1 \mathrm{~m} \times 2,5 \mathrm{~m}$ & 64 & $2,5 \mathrm{~m}^{2}$ & 4000 \\
$1,25 \mathrm{~m} \times 2,5 \mathrm{~m}$ & 16 & $3,125 \mathrm{~m}^{2}$ & 3200 \\
$1,5 \mathrm{~m} \times 2,5 \mathrm{~m}$ & 32 & $3,75 \mathrm{~m}^{2}$ & 2667 \\
$1,75 \mathrm{~m} \times 2,5 \mathrm{~m}$ & 16 & $4,375 \mathrm{~m}^{2}$ & 2286 \\
$2 \mathrm{~m} \times 2,5 \mathrm{~m}$ & 32 & $5 \mathrm{~m}^{2}$ & 2000 \\
$2,25 \mathrm{~m} \times 2,5 \mathrm{~m}$ & 16 & $5,625 \mathrm{~m}^{2}$ & 1778 \\
$2,5 \mathrm{~m} \times 2,5 \mathrm{~m}$ & 32 & $6,25 \mathrm{~m}^{2}$ & 1600 \\
$2,75 \mathrm{~m} \times 2,5 \mathrm{~m}$ & 16 & $6,875 \mathrm{~m}^{2}$ & 1455 \\
$3 \mathrm{~m} \times 2,5 \mathrm{~m}$ & 32 & $7,5 \mathrm{~m}^{2}$ & 1333 \\
\hline
\end{tabular}


In jeder Baumartenparzelle wurden drei Methoden der Begleitwuchsregulierung durchgeführt (Kontrolle; Mähen; Nutzpflanzen), zusätzlich wurde den Pflanzen in jeder zweiten Reihe Bodenhilfsstoffe bei der Pflanzung appliziert, so dass vier Varianten der Hilfsstoffapplikation angewendet wurden (Kontrolle; Hydrogel; Mykorrhiza; Hydrogel/Mykorrhiza) (Abb.3.3).

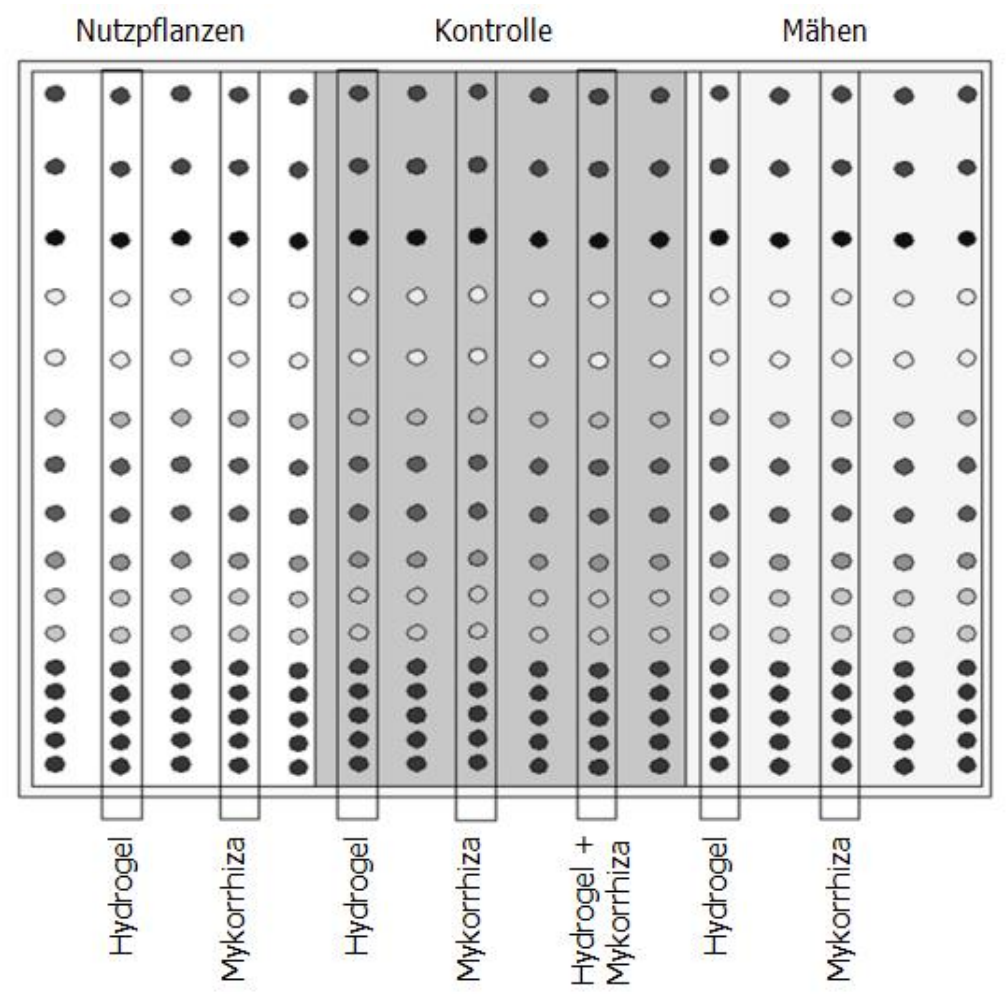

Abb. 3.3: Parzellenaufbau je Baumart auf der Versuchsfläche Sudheide in Gütersloh

\subsubsection{Begleitwuchsregulierung}

Pro Baumart wurde die Konkurrenzvegetation auf drei unterschiedliche Methoden reguliert. Auf der Ackerfläche wurde ein Drittel der Fläche als Kontrollfläche ohne Regulierungsmaßnahmen belassen. Auf dem zweiten Drittel wurde in der Vegetationsperiode alle zwei Monate zwischen den Pflanzenreihen gemäht. Zusätzlich wurde im Juni der ersten Vegetationsperiode die Konkurrenzvegetation direkt um die Pflanzen herum per Hacke entfernt. Auf dem letzten Drittel wurden, direkt nachdem die 
Gehölze gepflanzł wurden, der Boden zwischen den Pflanzreihen aufgeeggt, die Nutzpflanzen flächig ausgesät und anschließend angewalzt.

Die Auswahl der Nutzpflanzen-Mischung wurde in Anlehnung an die Untersuchungen von Denecke (1988) getroffen. Laut diesem haben sich auf Freiflächen sehr niedrige Kleemischungen aus Erd-, Faden-, Gelb- und Weißklee als sehr geeignet erwiesen. Zusätzlich wird empfohlen, noch Dauerlupine, Rübsen und Buchweizen in diese Mischung aufzunehmen. Da sowohl Daverlupine als auch Rübsen als Stickstoffbinder (Buchner, 1987) einen erhöhten Nitrataustrag bewirken können, wurde auf deren Einsatz verzichtet. Die auf den Flächen in Gütersloh ausgebrachte Saatgutmischung ist in Tab. 3.3 aufgelistet. Pro Quadratmeter wurden etwa 18 Buchweizensamen, 11 Erd-, 91 Faden-, 30 Gelb- und 63 Weißkleesamen ausgesät.

Tab. 3.3: Saatgutmischung für die Nutzpflanzendecke auf der Fläche Sudheide (Gütersloh)

\begin{tabular}{llll}
\hline Botanischer Name & Deutscher Name & Aussaatmenge [kg/ha] & Samenzahl/m \\
\hline Fagopyrum esculentrum & Buchweizen & 35 & 18 \\
Trifolium subterraneum & Erdklee & 9 & 11 \\
Trifolium minus & Fadenklee & 5 & 91 \\
Medicago lupulina & Gelbklee & 5 & 30 \\
Trifolium repens & Weißklee & 5 & 63 \\
\hline
\end{tabular}

Auf der Grünlandfläche wurden die Pflanzreihen im November 2006 vor der Pflanzung mit einem Totalherbizid (Roundup ${ }^{\circledR}$ UltraMax, Monsanto Agrar Deutschland GmbH, Düsseldorf, Deutschland) behandelt.

Roundup UltraMax ist ein nicht selektives Blattherbizid mit systemischer Wirkung, welches sich zu etwa 51 Gewichts-\% aus dem Wirkstoff Isopropylaminsalz von Glyphosat, 7,5 Gewichts-\% Netzmittel und 41,5 Gewichts-\% Wasser zusammensetzt. Das Herbizid wird über die grünen Teile der Pflanze aufgenommen und mit Hilfe des Saftstromes in der gesamten Pflanze, einschließlich der unterirdischen Pflanzenteile (Rhizome), verteilt, wodurch neben einjährigen auch mehriährige Unkraut- und Ungrasarten nachhaltig bekämpft werden (Monsanto Agrar Deutschland $\mathrm{GmbH}$ ). 
Die zukünftigen Pflanzreihen wurden mit einem Abstand von 2,5 $\mathrm{m}$ in einer Breite von 0,5 m gespritzł, so dass die Breite der unbehandelten Zwischenreihen $2 \mathrm{~m}$ beträgt. Somit erfolgte eine Behandlung von 20 \% der Grünlandfläche mit Herbiziden. Es wurden $4 \mathrm{l} /$ ha Roundup verwendet in einem Mischungsverhältnis Roundup : Wasser von 1 : 4. Vor der Pflanzung wurden die im November gespritzten Pflanzstreifen gepflügt, um die anschließenden Pflanzarbeiten zu erleichtern.

Die Begleitvegetation wurde auf der Grünlandfläche auf dieselbe Weise reguliert wie auf der Ackerfläche. Ein Drittel der Fläche wurde als Kontrollfläche belassen. Auf dem zweiten Drittel wurde in der Vegetationsperiode alle zwei Monate zwischen den Pflanzreihen gemäht und in der ersten Vegetationsperiode wurde einmal direkt um die Gehölze herum die Konkurrenzvegetation per Hacke entfernt. Auf dem letzten Drittel wurden, direkt nach der Pflanzung, Nutzpflanzen in die vegetationsfreien Pflanzstreifen gesät. Das Anwalzen der Samen entfiel auf der Grünlandfläche aufgrund der Gehölze.

\subsubsection{Hilfsstoffe (Mykorrhiza/Hydrogel)}

In jeder zweiten Pflanzreihe wurde den Gehölzen Hydrogel, Mykorrhiza oder eine Kombination aus beiden Hilfsstoffen beigegeben (Abb.3.3), um mögliche positive Auswirkungen auf den Anwuchserfolg der Pflanzen zu prüfen. Pro Begleitwuchsregulierung (Kontrolle, Mähen, Nutzpflanzen) wurde eine Pflanzreihe mit Hydrogel und eine mit Mykorrhiza behandelt. Zusätzlich fand in der Teilfläche ohne Begleitwuchsregulierung eine Kombination der beiden Hilfsstoffe statt (Abb. 3.3).

\subsubsection{Mykorrhiza}

Zur Mykorrhizierung der Steckhölzer und wurzelnackten Pflanzen wurde Amykor ${ }^{\circledR}$ Wurzel-Vital-Dip (Amykor GmbH, Greppin, DE) verwendet. Dieses besteht aus Amykor ${ }^{\circledR}$ Wurzel-Vitalpulver mit einem vesikulär-arbuskulären Mykorrhizapilz (VAMykorrhiza) und 5\% Hydrogelpulver. Bei dem VA-Mykorrhizapilz handelt es sich um 
Glomus intraradices. Durch Zugabe von Wasser erhält man eine Suspension, in die man die Stecklinge eintauchen kann. Durch die gelartige Konsistenz bleibt das mykorrhizahaltige Pulver am Steckling bzw. an der Wurzel kleben. Die Konsistenz der Suspension kann durch Wasserzugabe reguliert werden. Als Richtwert empfiehlt der Hersteller für $1000 \mathrm{ml}$ Amykor ${ }^{\circledR}$ Wurzel-Vital-Dip die Zugabe von 4-5 I Wasser. $20 \mathrm{ml}$ Amykor ${ }^{\circledR}$ Wurzel-Vital-Dip reichen dabei für etwa 10 Stecklinge bzw. Pflanzen aus. Für das Eintauchen der Stecklinge hatte die auf diese Weise angerührte Lösung die richtige Konsistenz, aber für die bewurzelten Pflanzen war diese zu dickflüssig. Deshalb wurde für die bewurzelten Pflanzen die doppelte Menge Wasser verwendet, so dass die gesamte Wurzeloberfläche mit der Suspension benetzt werden konnte. Die geringere Lösungskonzentration wird durch die wesentlich größere Benetzungsoberfläche der bewurzelten Pflanzen gegenüber den Stecklingen wieder ausgeglichen.

\subsubsection{Hydrogel}

Laut Hersteller sind $2 \mathrm{~kg}$ Stockosorb (Gefa Produkte Fabritz GmbH, Krefeld, DE) einem Kubikmeter Bodensubstrat beizumischen. Als Bodenaushub ergab sich für einen Pflanzlochbohrer mit $20 \mathrm{~cm}$ Durchmesser und einer Bohrtiefe von $19 \mathrm{~cm}$ ein Bodenaushub von $6 \mathrm{I}$. Je zu behandelnder Pflanze wurden die 6 I Bodensubstrat in einen Eimer gefüllt, ca. $12 \mathrm{~g}$ Stockosorb ${ }^{\circledR} 500$ micro mit dem Substrat vermischt und die Stecklinge/ Pflanzen mit der Substratmischung eingepflanzt.

\subsection{Versuchsfläche Hamerstorf (Landkreis Uelzen)}

\subsubsection{Standortsbeschreibung}

Die untersuchte Fläche Hamerstorf (52 $54^{\prime} 30.00^{\prime \prime} N$; $\left.10^{\circ} 28^{\prime} 2.00^{\prime \prime} E\right)$ befindet sich in dem Wuchsgebiet ostniedersächsisches Tiefland am westlichen Rand des 
Wuchsbezirkes Ost Heide. Die Höhenlage bewegt sich bei 40 bis $50 \mathrm{~m}$ ü. NN in der planaren Höhenstufe. Die Klimadaten der Wetterstation Uelzen von 1961-1990 ergeben eine mittlere Jahrestemperatur von $8,5{ }^{\circ} \mathrm{C}$ und einen mittleren Jahresniederschlag von $622 \mathrm{~mm}$. Der kälteste Monat ist der Januar mit durchschnittlich $0{ }^{\circ} \mathrm{C}$, der wärmste der Juli mit $16,8^{\circ} \mathrm{C}$. (Quelle: DWD)

Die 1,6 ha große mit Pappeln bestockte Fläche war vorher Brache. Sie ist mit einer landwirtschaftlichen Bodenzahl von 30 bewertet. Im Süden schließt sich eine im selben Jahr (2006) mit Weide begründete Kurzumtriebsplantage an. Die Pappelfläche ist leicht nach Norden geneigt, wo sie in einer Senke endet. Der Bodentyp verändert sich in Richtung der Senke von einer Podsol-Braunerde zu einer Gley-Braunerde.

Aufgrund der Voruntersuchungen von Lamersdorf (2008) vor der Kulturanlage und des eher armen bodenkundlich-geologischen Ausgangsmaterials (Braunerde-Gley über Schmelzwasser-/Auesanden) wurde die Fläche als potentieller Mangelstandort ausgewiesen, da die N-Verfügbarkeit relativ niedrig war. Die hohen pflanzenverfügbaren Vorräte an $\mathrm{Ca}$ wurden vermutlich durch die vormalige Aufkalkung aus der landwirtschaftlichen Nutzung verursacht. Die Gehalte und austauschbaren Vorräte an Kalium (K) und Magnesium (Mg) waren mäßig bis gering.

\subsubsection{Flächenplan}

In Hamerstorf untergliederte sich die im Frühjahr 2006 begründete Kultur in eine ehemalige Grünbrache, welche mit Pappeln begründet wurde, und eine Ackerfläche, die mit Weide bestockt wurde (Abb. 3.4). Auf der Grünlandbrache wurden drei unterschiedliche Flächenvorbehandlungen durchgeführt:

- gepflügt und geeggt

- nur gepflügt

- Herbizidbehandlung 


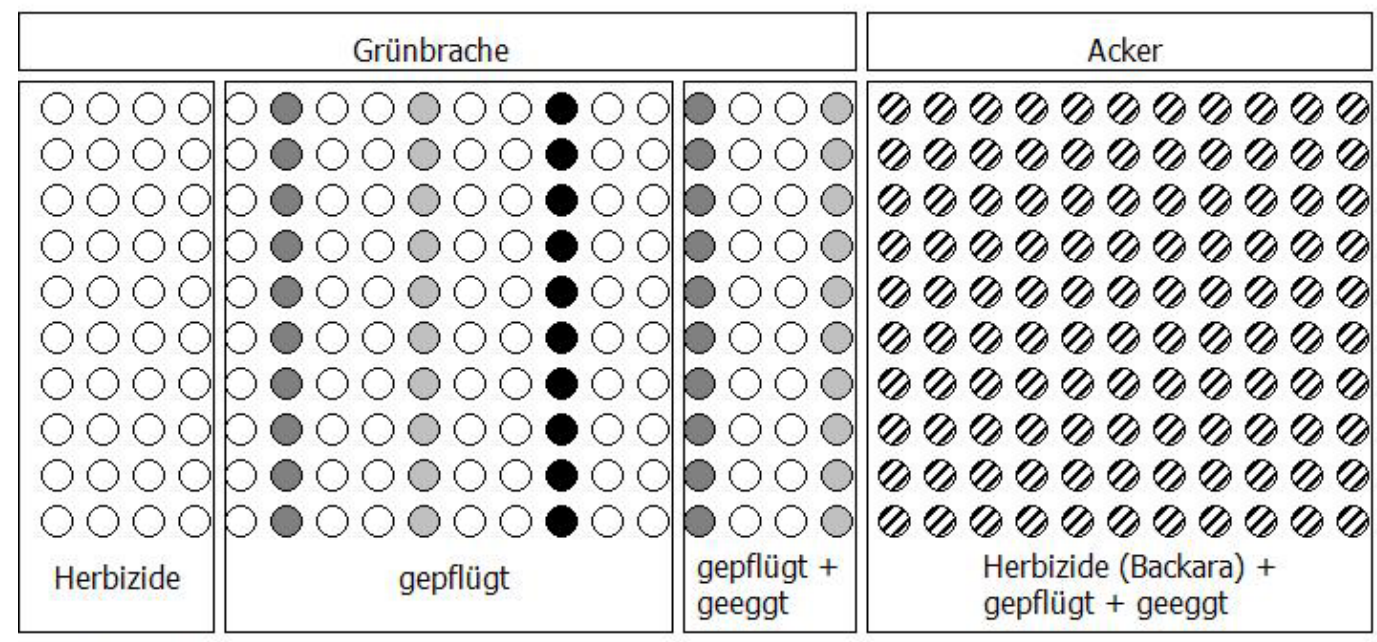

Pappel "Androscoggin"

Pappel "NE 42"

Pappel "Max 4"

- Pappel "Weser 6"

Weide "Tora"

\section{Abb. 3.4: Flächenaufbau in Hamerstorf (Uelzen)}

Am 20. April 2006 wurden die mechanisch bearbeiteten Flächen mit einem vier Schar-Volldrehpflug mit Packer (Kombination von Walzen) auf eine Pflugsohle von 25 $\mathrm{cm}$ gepflügt. Auf der nur gepflügten Fläche wurde auf den Einsatz des Packers verzichtet. Die Herbizidbehandlung der dritten Teilfläche wurde bereits Ende März 2006 Roundup TTURBO (600 g/kg Glyphosat, Monsanto Agrar Deutschland GmbH) nach Herstellerangabe durchgeführt.

Es wurden vier unterschiedliche Pappelhybriden für die Flächenanlage ausgewählt, und zwar "Androscoggin", "Max 4", "NE 42" und "Weser 6" (Tab. 3.4). Die Pappeln wurden im Pflanzverband 2 × 2 m mit 2500 Pflanzen/ha gepflanzt.

Tab. 3.4: Auf der Versuchsfläche Hamerstorf bei Uelzen gepflanzte Pappelstecklinge

\begin{tabular}{l|l}
\hline Bezeichnung & Kreuzungskombination \\
\hline "Androscoggin" & P. maximowiczii $\times$ P. trichocarpa \\
"Max 4" & P. nigra x P. maximowiczii \\
"NE 42" & P. maximowiczii x P. trichocarpa \\
"Weser 6" & P. trichocarpa \\
\hline
\end{tabular}


Auf daneben gelegener Ackerfläche, welche vor der Pflanzung gespritzt, gepflügt und geeggt wurde, wurde die Weidensorte "Tora" (Salix viminalis x S. schwerinii) 13.000 Pflanzen/ha angebaut. Der Reihenabstand betrug $75 \mathrm{~cm}$ in der Doppelreihe und 1,50 m zwischen den Doppelreihen. In der Reihe betrug der Pflanzenabstand etwa $65 \mathrm{~cm}$.

\subsection{Versuchsfläche Unterlüß (Landkreis Uelzen)}

\subsubsection{Standortsbeschreibung}

Unterlüß liegt im Naturpark Südheide in einer Höhe von etwa 95 Metern über NN auf einer eiszeitlichen Geestfläche, die im Westen und Südwesten bis auf $118 \mathrm{~m}$ bzw. $116 \mathrm{~m}$ ansteigt (Richter, 1984). Das Gebiet um Unterlüß gehört als Teil der Südheide großklimatisch zum offenen norddeutschen Flachlandraum, in dem kleinklimatische Besonderheiten kaum auftreten (Richter, 1984). Das Klima wird im Wesentlichen durch atlantische Luftmassen bestimmt, die meist wechselhaftes Wetter hervorrufen, doch lassen sich auch einzelne kontinentale Klimakomponenten erkennen (Richter, 1984).

Die durchschnittliche Lufttemperatur beträgt $8,1{ }^{\circ} \mathrm{C}$ im Jahresmittel. Der kälteste Monat ist der Januar mit durchschnittlich $-0,2{ }^{\circ} \mathrm{C}$, der wärmste der Juli mit $16,5{ }^{\circ} \mathrm{C}$. Die mittlere Jahresschwankung beträgt somit $16,7{ }^{\circ} \mathrm{C}$. Die Vegetationszeit mit einer Tagesmitteltemperatur von mindestens $10{ }^{\circ} \mathrm{C}$ beginnt im Mittel am 03. Mai und endet am 09. Oktober. Die mittlere Jahressumme der Niederschläge beträgt $805 \mathrm{~mm}$ (1961-1990). (Quelle: DWD)

Auf der Versuchsfläche Unterlüß Abteilung 229 c (5259'0.00"N; 10¹5'25.00"E) herrschen mäßig bis nachhaltig frische, ärmere Sande vor (Renner, 2002). Vorherrschende Bodenart in der Gemeinde Unterlüß ist Podsol-Braunerde (Gunreben \& Boess, 2008). 


\subsubsection{Flächenplan}

Bei Abt. 229 c in Unterlüß handelt es sich um einen jahrzehntelang extensiv bewirtschafteten Wildacker, welcher vor der Flächenbegründung auf $30 \mathrm{~cm}$ gepflügt wurde. Bei den verwendeten Pappelhybriden handelte es sich um "Max 2", "Max 3", "NE 42" und "Weser 6".

Tab. 3.5: Auf der Versuchsfläche Unterlüß bei Uelzen gepflanzte Pappelstecklinge

\begin{tabular}{l|l}
\hline Bezeichnung & Kreuzungskombination \\
\hline "Max 2" & P. nigra x P. maximowiczii \\
"Max 3" & P. nigra x P. maximowiczii \\
"NE 42" & P. maximowiczii x P. trichocarpa \\
"Weser 6" & P. trichocarpa \\
\hline
\end{tabular}

In einzelnen Pflanzreihen wurden die Stecklinge bei der Pflanzung mit Wurzel-Vitaldip mykorrhiziert (siehe Kapitel 3.1.4). Der Pflanzverband beträgt 2 × 1,50 m. Im Mai 2007 wurde die Fläche begründet und im August desselben Jahres eingezäunt.

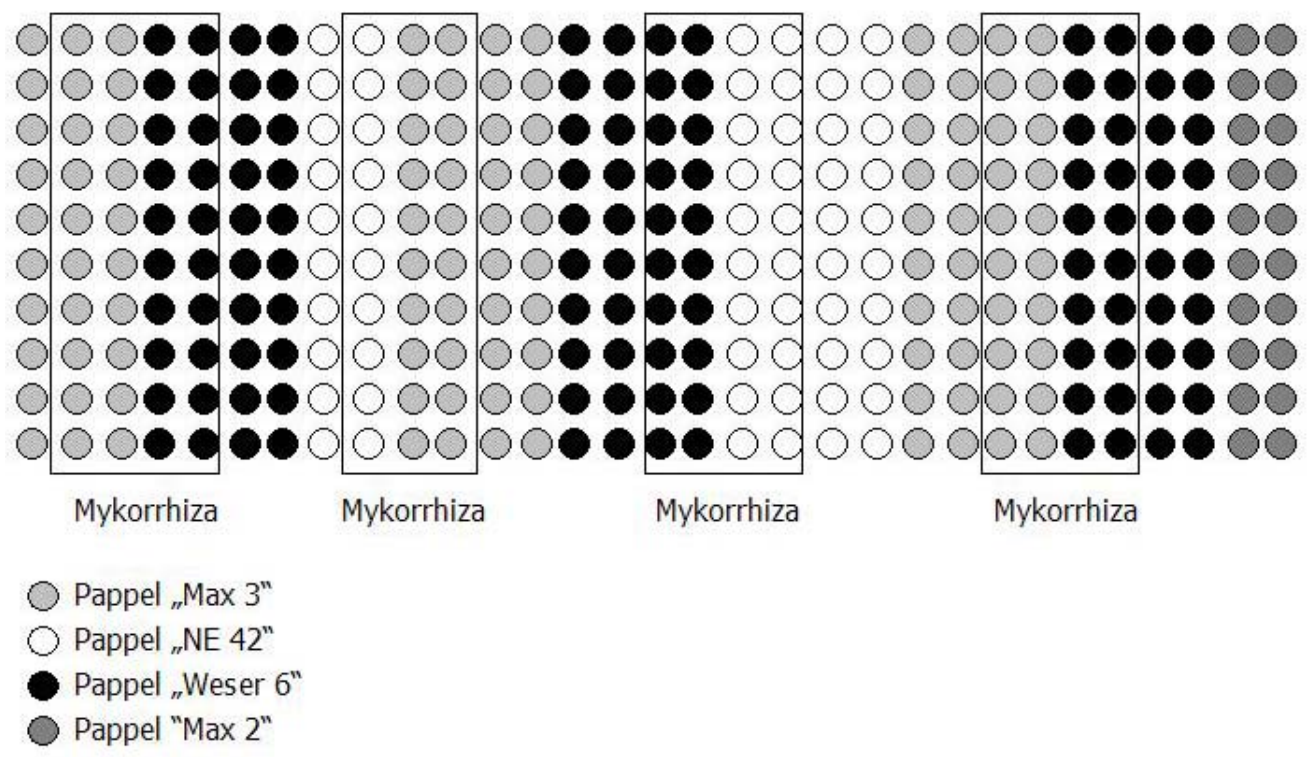

Abb. 3.5: Flächenaufbau in Unterlüß (Uelzen) 


\subsection{Untersuchungsmethoden}

\subsubsection{Sickerwasseruntersuchung}

Mit einem Lysimeter werden Quantität und Qualität des Bodensickerwassers bestimmt. In der Landwirtschaft und Umweltforschung werden Lysimeter zur Erfassung von Wechselwirkungen bzw. Stofftransporten zwischen der Atmosphäre, den Pflanzen, dem Boden, der Tierwelt und dem Grundwasser verwendet.

Der Einbau der ersten Lysimeter erfolgte auf der Versuchsfläche Sudheide in Gütersloh zusammen mit dem Abstecken der Teifflächen Ende Oktober 2006. Es wurde insgesamt acht Lysimeter auf der Fläche installiert. Dabei handelt es sich um eine $65 \mathrm{~cm}$ lange Röhre mit einer durchlässigen Saugkerze am unteren Ende, die mittels Bohrstock etwa $70 \mathrm{~cm}$ tief in den Boden versenkt wurden. Jedes Lysimeter ist per PVCSchlauch mit einer Glasflasche verbunden, die sich in einer Kühlbox befindet. Die Kühlboxen, in den sich je zwei Flaschen befinden, wurden bis zum Deckel eingegraben, und auch die PVC-Schläuche wurden zum Schutz vor Algenbildung etwa $2 \mathrm{~cm}$ unter der Bodenoberfläche verlegt. Direkt nach der Installation wurde mittels einer Elektropumpe ein Unterdruck von 0,6 bar in den Systemen erzeugt.

Die Lysimeter wurden an der südlichen Flächenbegrenzung, an der Grenzlinie zwischen Acker- und Grünlandfläche eingebaut. Zwei Lysimeter befinden sich auf der Ackerfläche, drei auf der unbehandelten Grünfläche und drei Lysimeter wurden auf einem gefrästen Teil der Grünlandfläche installiert. Während zwischen gefrästen und ungefrästen Grünland die deutlichsten Unterschiede der Wasserwerte erwartet werden, werden auf der Ackerfläche nur geringe Abweichungen erwartet, weshalb zwei Lysimeter als ausreichend für die Bewertung der Wasserqualität erachtet werden. Die Lysimeter werden in nur geringen Abständen voneinander in den Boden gesetzt, um kleinstandörtliche Unterschiede so gering wie möglich zu halten.

Mitte Juni 2007 wurden weitere sechs Lysimeter auf der Ackerfläche zwischen den Weidenstecklingen eingebaut. Zwei Lysimeter wurden auf der unbehandelten Teilfläche ohne Begleitwuchsregulierung, zwei auf der Fläche mit Nutzpflanzeneinsaat 
und zwei auf der von Unkraut befreiten Teiffläche eingebaut. Der Einbau erfolgte jeweils bei Kontroll-Stecklingen ohne Zusatz von Hilfsstoffen, die in einem Abstand von $1 \mathrm{~m}$ zueinander gesetzt wurden.

\subsubsection{Bodensaugspannung}

Die Bodensaugspannung gibt Aufschluss über die Austrocknung des Bodens und wurde auf der Versuchsfläche Sudheide in Gütersloh mit Hilfe von Tensiometern gemessen. Je trockener ein Boden ist, desto größer ist die gemessene Saugspannung im Boden. Die Saugspannung wird in $\mathrm{hPa}$ angegeben. Der Einbau der Tensiometer erfolgte in den Tiefenstufen 10, 30 und $70 \mathrm{~cm}$. Sowohl auf der Ackerfläche als auch auf der Grünlandfläche wurden Tensiometer der drei Tiefenstufen zwischen die Weide "Turbo" eingebaut. Die Messung der Bodensaugspannung erfolgt bei allen drei Maßnahmen zur Begleitwuchsregulierung (Mähen, Nutzpflanzeneinsaat, Kontrolle). Die Messungen der Bodensaugspannung erfolgten ab Juli 2007 in zweiwöchigem Rhythmus, ab August 2008 in einem vierwöchigen Rhythmus.

\subsubsection{Vegetationsaufnahme}

Für die Vegetationsaufnahmen wurden insgesamt Probeflächen von $1 \mathrm{~m}^{2}$ in die Fläche gelegt und der Deckungsgrad der Arten bestimmt. Von den Hauptarten wurden dann Pflanzenproben für die Bestimmung der oberirdischen Biomasse genommen. Hierfür wurden Flächen der Größe $20 \times 20 \mathrm{~cm}$, welche nur von einer Art bedeckt waren, abgeerntet, bei $35^{\circ} \mathrm{C}$ getrocknet und die Trockenbiomasse bestimmt. Die Erhebung der Biomasse bei $100 \%$ iger Deckung lässt es zu, die Trockenbiomasse der Probeflächen bei geringerem Deckungsgrad zu berechnen. Anhand der Probeflächen wurde die Biomasse der Probeflächen wurde auf den Hektar hochgerechnet. Die Vegetationsaufnahmen erfolgten jeweils im Monat Juli. 
In Gütersloh wurden in der ersten und zweiten Vegetationsperiode $(2007 ; 2008)$ der Deckungsgrad und die Biomasse der Begleitvegetation geschätzt. Insgesamt wurden 30 Probeflächen angelegt. Pro Vornutzung (Acker; Grünland) und Begleitwuchsregulierung (Kontrolle; Mähen; Nutzpflanzeneinsaat) wurden jeweils fünf Wiederholungen durchgeführt.

Auf der Fläche Hamerstorf wurden je Bearbeitungsverfahren (gepflügt und geeggt, gepflügt, gespritzt) ebenfalls fünf Wiederholungen mit insgesamt 15 Probeflächen angelegt. Die Vegetationsaufnahme erfolgte in der zweiten und dritten Vegetationsperiode (2007; 2008).

Auf der Versuchsfläche Unterlüß wurde die Vegetationsaufnahme in der ersten Vegetationsperiode (2007) mit ebenfalls 5 Wiederholungen durchgeführt.

\subsubsection{Anwuchs-/Vitalitätsbewertung}

Zeitgleich mit der Begleitvegetation wurden der Anwuchs und die Vitalität der Gehölze aufgenommen.

Der Anwuchserfolg und die Vitalität der Gehölze wurde im Juli 2007 und 2008 auf der Flächen Sudheide in einer Vollaufnahme bewertet.

Auf der Fläche Hamerstorf wurde die Vitalität der Pappeln in der zweiten und dritten Vegetationsperiode in einer Stichprobe bewertet (2007; 2008). Auf der Fläche Unterlüß wurde in der ersten Vegetationsperiode (2007) die Anzahl der ausgetriebenen Stecklinge ebenfalls in einer Stichprobe aufgenommen. Als Probefläche wurde sowohl in Hamerstorf als auch in Unterlüß ein Rechteck angelegt, welches in Hamerstorf etwa $20 \%$ und in Unterlüß etwa $10 \%$ der Gesamtfläche entspricht. Dies entspricht in Hamerstorf 20 Reihen und in Unterlüß 31 Reihen mit jeweils 10 Pflanzen pro aufgenommener Reihe.

Für Bewertung der Vitalität wurde folgende Skala verwendet:

1 - vitale Triebe, keine erkennbaren Schäden

2 - geringere Wuchsleistung, vereinzelte Schäden, gute Regenerationsfähigkeit 
3 - teils abgestorbene Pflanzenteile, Regenerationsfähigkeit möglich

4 - fast völlig abgestorbene Gehölze, Regenerationsfähigkeit kaum gegeben

5 - völlig abgestorbene Pflanzen

\subsubsection{Zuwachsmessungen}

Auf der Fläche Sudheide in Gütersloh wurde direkt nach der Pflanzung an $10 \%$ der Pflanzen die durchschnittliche Ausgangshöhe der gelieferten Baumschulpflanzen ermittelt. Jeweils am Ende der ersten beiden Vegetationsperioden (2007; 2008) wurden die Höhen und Wurzelhalsdurchmesser in einer Vollaufnahme gemessen.

Auf der Fläche Hamerstorf (Uelzen) wurden Höhe und Wurzelhalsdurchmesser der Pappelstecklinge nach Ende der zweiten und dritten Vegetationsperiode erfasst (2007; 2008). Die Messungen erfolgten auf denselben Probeflächen wie für die Anwuchs- und Vitalitätsbewertung.

Auf der Fläche Unterlüß (Uelzen) erfolgte am Ende der ersten Vegetationsperiode (2007) eine Höhenmessung der Pappelstecklinge. Diese wurde auf derselben Probefläche wie die Anwuchs- und Vitalitätsaufnahme durchgeführt.

\subsubsection{Nährstoffanalyse}

Von den Gehölzen auf der Versuchsfläche Sudheide bei Gütersloh wurden im Juli 2008, der zweiten Vegetationsperiode, ausgewählte Blattproben zur Nährstoffanalyse genommen. Pro gepflanzte Baumart wurde von 4 Kontrollpflanzen Blattproben genommen, um einen allgemeinen Überblick über die Nährstoffversorgung der verschiedenen Baumarten zu gewinnen. Zusätzlich wurde auf der Ackerfläche, ebenfalls mit jeweils 4 Wiederholungen, von dem Bergahorn, dem Pappelsteckling "Max 4" und dem Weidenstecklung "Turbo" Blattproben der unterschiedlich durchgeführten Varianten (Kontrolle, Mähen, Nutzpflanzen, Hydrogel, Mykorrhiza, Hydrogel + Mykorrhiza) genommen, um deren Auswirkungen auf die 
Nährstoffversorgung im Vergleich zu den Kontrollpflanzen bewerten zu können. Der Pappelsteckling "Max 4" und der Weidensteckling "Turbo" wurden für die eingehendere Untersuchung ausgewählt, da diese als typische Baumarten auf Kurzumtriebsplantagen gepflanzt werden. Bergahorn repräsentiert eine mehr waldtypische Baumart, die für Kurzumtriebsplantagen eher selten in Betracht gezogen wird, aber ökologische Vorteile bieten kann.

\subsubsection{Statistische Auswertung}

Die statistische Auswertung der Daten wurde mit Excel und dem Add-In Winstat durchgeführt. Von den metrisch skalierten Daten wurden Mittelwerte und Standardfehler der Wiederholungen einer Gruppe berechnet. Von nicht metrischen Daten, von denen mehrere Wiederholungen aufgenommen wurden, wurden Häufigkeiten berechnet und aus den Häufigkeitswerten der Wiederholungen dann Mittelwert und Standardfehler berechnet. Bei nicht metrischen Daten, von denen es keine Wiederholungen gab, dürfen nur Häufigkeiten berechnet werden. Bei der Vitalitätseinstufung wurden, obwohl es sich um ordinal skalierte Daten handelt, Mittelwert und Standardabweichung der Daten berechnet.

Die Werte von verschiedenen Gruppen wurden auf signifikante Unterschiede überprüft. Zum Mittelwertvergleich von zwei unterschiedlichen Gruppen mit metrisch skalierten Daten wurde der T-Test durchgeführt Dazu wurde der sogenannte F-Test als Vortest durchgeführt, um zu sichern, dass die Stichproben normalverteilten Grundgesamtheiten entstammen.

Der Vergleich mehrerer Gruppen mit metrischen Werten erfolgte per Varianzanalyse. Generell wird bei diesem Verfahren untersucht, ob Mittelwerte von Grundgesamtheiten, denen Stichproben entnommenen wurden, als gleich anzunehmen sind. Eine Voraussetzung für die Varianzanalyse, oft auch als ANOVA (analysis of variance) bezeichnet, besteht darin, dass die Daten auf homogenen Grundgesamtheiten basieren. Um dies zu untersuchen, wird von WinSTAT zugleich 
automatisch der Barlett-Test durchgeführt, ein c²-Test zur Prüfung auf Gleichheit der Varianzen.

Beim Vergleich von zwei Gruppen mit nicht metrischen Daten wurde der U-Test (MannWhitney) durchgeführt. Im Prinzip unterscheidet sich der U-Test kaum vom t-Test für unabhängige Stichproben. WinSTAT zeigt dies unter anderem am aufgerufenen Dialog U-Test, der lediglich eine andere Bezeichnung in der Titelzeile führt als der Dialog t-Test. Der eigentliche Unterschied zwischen beiden Verfahren besteht aber darin, dass der t-Test ein parametrisches und der U-Test ein nicht-parametrisches Verfahren darstellt. Damit lässt sich der U-Test unabhängig von der Verteilungsart anwenden und alle Überlegungen zur Normalverteilung der Daten entfallen. Somit wird dieser Test zu einem Mittel der Wahl, wenn Tests, die auf Normalverteilung aufbaven, nicht zulässig sind.

Bim Vergleich mehrerer Gruppen mit nicht metrisch skalierten Werten wurde der H-Test verwendet. Dieses auch mit Kruskal-Wallis-Test bezeichnete Verfahren ähnelt der einfaktoriellen Varianzanalyse, bedient sich aber der Rangfolge der Messwerte. Damit wird dieser Test zum großen Bruder des U-Tests, der jeweils nur zwei Stichproben aufgrund der Ränge vergleicht. Ebenso wie beim U-Test handelt es sich beim H-Test folglich um ein parameterfreies Prüfverfahren für ordinalskalierte, stetige Zufallsvariablen, so dass auch hier keine weiteren Voraussetzungen bezüglich Art und Form der Verteilung erfüllt sein müssen. Es wird geprüft, ob zwei oder mehr Stichproben einer gemeinsamen Grundgesamtheit entstammen. Ist dies der Fall, bedeutet dies zugleich, dass die Stichproben zusammengenommen derselben Verteilung genügen. So lässt sich z. B. mit diesem Test die Frage klären, ob Stichproben vereinigt werden dürften.

Die Verfahren der Regressions- und Korrelationsanalysen dienen der Beschreibung eines Zusammenhanges zwischen zwei (oder mehreren) Merkmalen. Die Regression beschreibt dabei die Art eines (gerichteten) Zusammenhanges (Je-Desto-Beziehung), die Korrelation misst die Stärke eines (ungerichteten) Zusammenhanges. 
Bei der Regressionsanalyse unterscheidet man zwischen einer abhängigen und einer (oder mehreren) unabhängigen Variablen. Ziel der Analyse ist es festzustellen, wie sich Änderungen der unabhängigen Variablen auf die abhängige Variable auswirken. Die Regressionsanalyse beschreibt also die Art des Zusammenhanges und ermöglicht über die reine Beschreibung hinaus eine Voraussage (Prädiktion). Zu den gegebenen Wertepaaren werden die entsprechenden Koeffizienten geschätzt. Die Regressionsgerade soll die zugrundeliegenden Daten so gut wie möglich repräsentieren. Als Maßzahl zur Beurteilung der Güte der Regressionsschätzung dient das Bestimmtheitsmaß $r^{2}$. Es stellt das Verhältnis von erklärter Streuung zur Gesamtstreuung dar $\left(0 \leq r^{2} \leq 1\right)$.

Die Korrelationsanalyse untersucht den (ungerichteten) Zusammenhang zweier gleichberechtigter Merkmale und quantifiziert die Stärke des Zusammenhangs. Sie wird eingesetzt, wenn man die Richtung des Zusammenhanges nicht kennt bzw. diese nicht von Interesse ist. Der Korrelationskoeffizient nach Pearson diente als Maß für den linearen Zusammenhang zwischen den Wertereihen zweier Stichproben. Hat er den Wert 0 , sind die Daten unkorreliert, hat er den Wert 1, besteht volle Übereinstimmung. Damit der Test durchführbar ist, müssen metrische, normalverteilte Daten vorliegen. Der Korrelationskoeffizient nach Pearson hängt funktional mit dem linearen Bestimmtheitsmaß $r^{2}$ aus der Regressionsanalyse zusammen. 


\section{Ergebnisse}

\subsection{Versuchsfläche Sudheide (Landkreis Gütersloh)}

\subsubsection{Klimadaten}

Von den Stadtwerken Gütersloh (SWG) wurden der Temperaturverlauf und die Niederschlagsmengen der Wasserwirtschaftsjahre 2007 (Abb. 4.1) und 2008 (Abb. 4.2Abb. 4.2) zur Verfügung gestellt. Ein Wasserwirtschaftsjahr gibt den Zeitraum von November bis Oktober des folgenden Jahres wieder. Dieser Zeitraum wird im Folgenden für die Berechnung der durchschnittlichen Jahrestemperatur und der Jahresniederschlagssumme verwendet (Tab. 4.1). Die Durchschnittstemperatur im Jahr der Kulturanlage (Nov. '06 - Okt. '07) lag bei $11,4{ }^{\circ} \mathrm{C}$, und damit 2,2 ${ }^{\circ} \mathrm{C}$ über der durchschnittlichen Jahresmitteltemperatur. Der Jahresniederschlag betrug $1008 \mathrm{~mm}$, also ca. $240 \mathrm{~mm}$ mehr als im langiährigen Mittel. Insgesamt handelte es sich also um ein besonders warmes und feuchtes Jahr. Bei näherer Betrachtung zeigt sich allerdings, dass direkt nach der Pflanzung (März 07) besonders trockene Bedingungen auf der Versuchsfläche vorherrschten. In den sieben Wochen nach der Kulturanlage fiel kaum Niederschlag. Insgesamt fielen in 50 Tagen nur 26 mm Niederschlag. Im April 2007 lag die Niederschlagsmenge bei $4 \mathrm{~mm}$ mit einer Durchschnittstemperatur von $13,6^{\circ} \mathrm{C}$. Die langiährige monatlichen Durchschnittstemperatur und Niederschlagsmenge im April liegt dagegen bei $8,4{ }^{\circ} \mathrm{C}$ und $55 \mathrm{~mm}$. Damit war gerade die kritische Anfangsphase nach der Kulturbegründung, welche für den Pflanzenanwuchs besonders wichtig ist, überdurchschnittlich warm und trocken. In der restlichen ersten Vegetationsperiode fielen dagegen umso mehr Niederschläge. 
Lufttemperatur

$\left[{ }^{\circ} \mathrm{C}\right]$

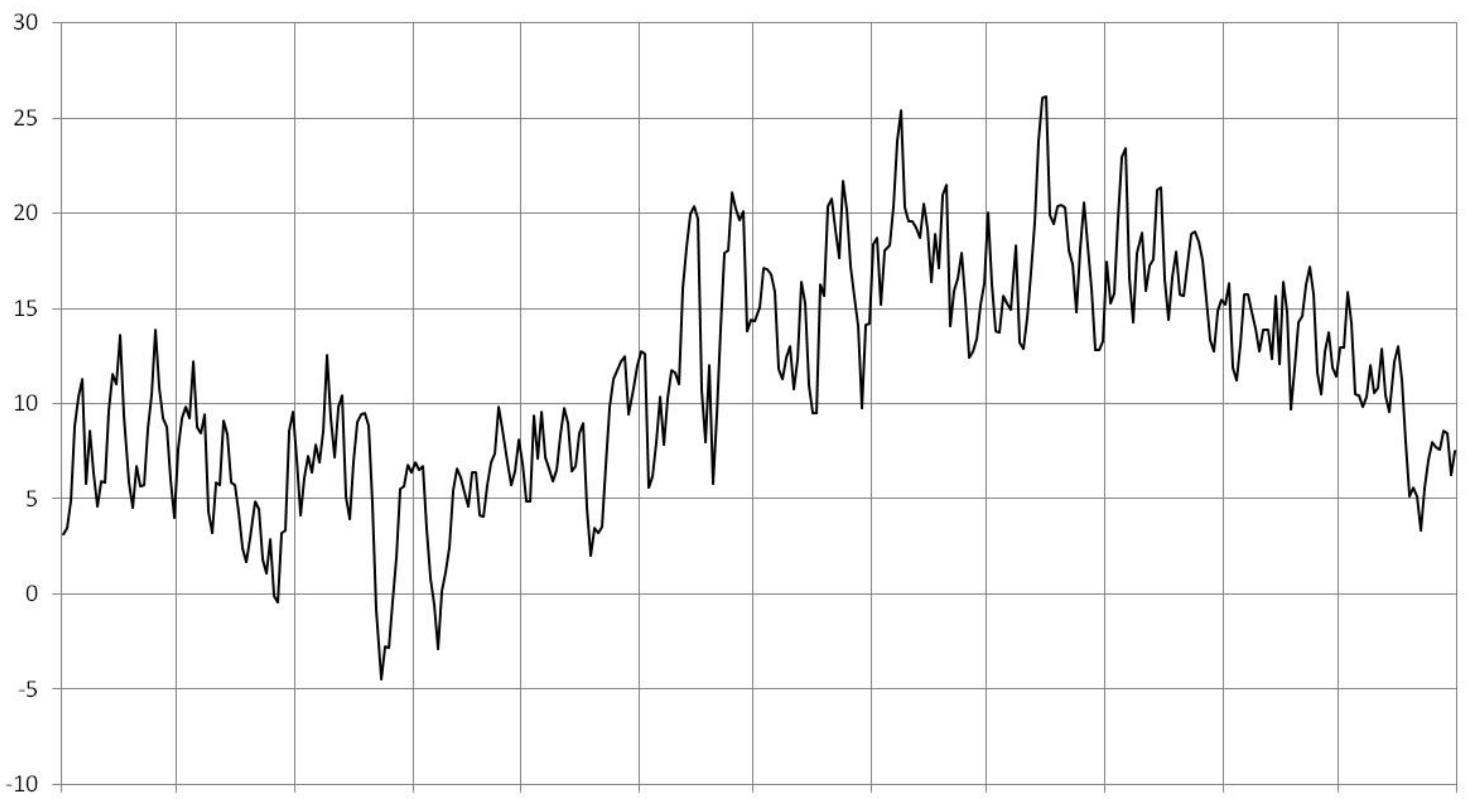

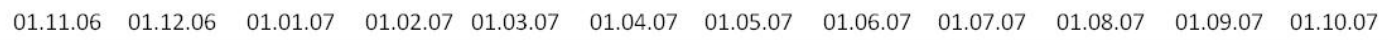

Niederschlagsmengen

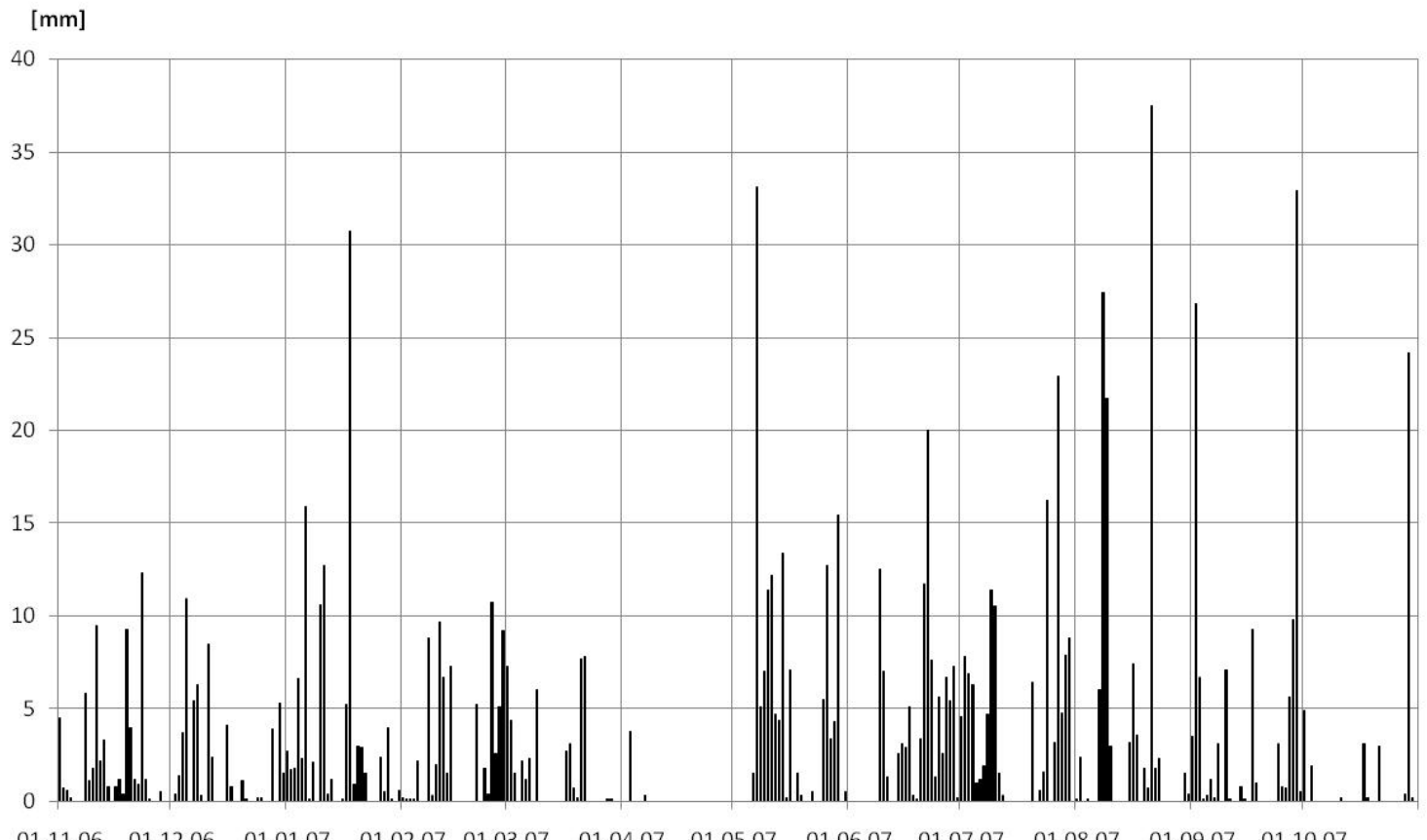

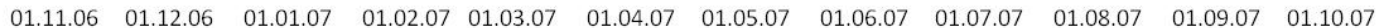

Abb. 4.1: Temperaturverlauf und Niederschlagsmengen im Wasserwirtschaftsjahr 2007 (Nov.06 - Okt.07) (Quelle: SWG) 

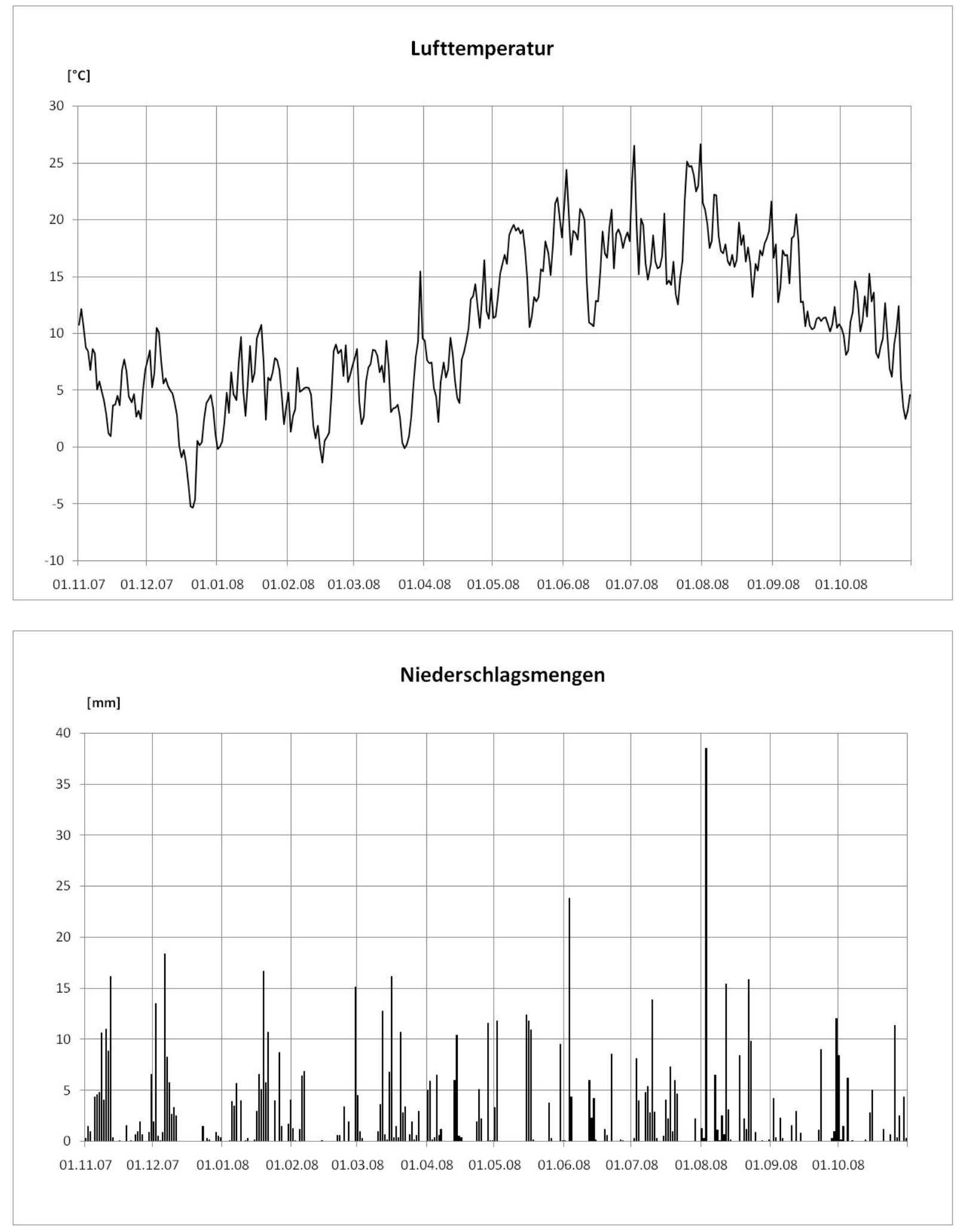

Abb. 4.2: Temperaturverlauf und Niederschlagsmengen im Wasserwirtschaftsjahr 2008 (Nov.07 - Okt.08) (Quelle: SWG)

Die Niederschlagsmenge im Wasserwirtschaftsjahr 2008 (Nov. '07 - Okt. '08) entsprach mit $773 \mathrm{~mm}$ in etwa dem langfristigen Mittel. Die mittlere Jahrestemperatur 
war mit $10,6{ }^{\circ} \mathrm{C}$ mehr als $1,4{ }^{\circ} \mathrm{C}$ höher als im Durchschnitt. Die durchschnittlichen Monatstemperaturen lagen im Mai mit $3,5^{\circ} \mathrm{C}$ und im Juni und Juli mit je $1,7{ }^{\circ} \mathrm{C}$ über den langiährigen Mittelwerten. Im Juli fiel 27 mm weniger Niederschlag, womit dieser Monat etwas trockener als im langiährigen Durchschnitt war.

Die Vegetationszeit daverte im ersten Jahr 210 Tage an, also 40 Tage länger als im Durchschnitt. Von Mitte März bis Mitte Oktober 2007 war die Lufttemperatur höher als $10{ }^{\circ} \mathrm{C}$. Im zweiten Kulturjahr 2008 davert die Vegetationszeit 180 Tage an (Mitte April - Mitte Oktober 2008), also 10 Tage länger als die durchschnittlichen 170 Tage.

Tab. 4.1: Monatliche Durchschnittstemperaturen und Niederschläge für Gütersloh (1961 - 1990) und der Wasserwirtschaftsjahre 2007 (Nov.'06 - Okt.'07) und 2008 (Nov.'07 - Okt.'08) (Quellen: DWD; SWG)

\begin{tabular}{|c|c|c|c|c|c|c|c|c|c|c|c|c|c|c|}
\hline & & Nov & Dez & Jan & Feb & Mrz & Apr & Mai & Jun & Jul & Aug & Sep & Okt & \\
\hline \multirow[b]{2}{*}{$\begin{array}{l}\frac{1}{\circ} \text { 영 } \\
2\end{array}$} & ${ }^{\circ} \mathrm{C}$ & 5,3 & 2,5 & 1,3 & 2,0 & 4,7 & 8,4 & 12,9 & 15,9 & 17,2 & 16,9 & 13,7 & 9,9 & $\varnothing 9,2$ \\
\hline & $\mathrm{mm}$ & 64,4 & 74,2 & 64,7 & 47,8 & 59,7 & 54,9 & 64,2 & 78,6 & 71,7 & 67,3 & 62,5 & 51,7 & $\sum_{761,7}$ \\
\hline \multirow{2}{*}{ 仓̊ } & ${ }^{\circ} \mathrm{C}$ & 7,9 & 5,6 & 5,7 & 5,0 & 7,8 & 13,6 & 15,1 & 18,0 & 17,6 & 17,3 & 13,7 & 9,5 & $\varnothing 11,4$ \\
\hline & $\mathrm{mm}$ & 62,4 & 56,5 & 110 & 74 & 47,3 & 4,1 & 144,2 & 106,7 & 130,5 & 120,9 & 113,7 & 38,1 & $\sum_{1008,4}$ \\
\hline \multirow{2}{*}{$\stackrel{\infty}{\stackrel{\sim}{~}}$} & ${ }^{\circ} \mathrm{C}$ & 5,7 & 2,9 & 5,5 & 4,4 & 5,7 & 9,0 & 16,4 & 17,6 & 18,9 & 18,0 & 13,5 & 9,6 & $\varnothing 10,6$ \\
\hline & $\mathrm{mm}$ & 81,4 & 61,7 & 85,7 & 37,6 & 72,7 & 58,1 & 64,1 & 51,7 & 70,5 & 108,4 & 36 & 45,3 & $\sum_{773,2}$ \\
\hline
\end{tabular}

\subsubsection{Sickerwasseruntersuchung}

Die Messungen des Nitratgehaltes im Sickerwasser zeigten, dass die Austräge von Stickstoff auf der ehemaligen Ackerfläche anfangs mit über 140 mg/l sehr hoch waren, aber nach Stilllegung der Flächen schnell abnahmen und unter den nach der Trinkwasserverordnung erlaubten Grenzwert von $50 \mathrm{mg} / \mathrm{I}$ sanken und auch blieben (Abb. 4.3). 


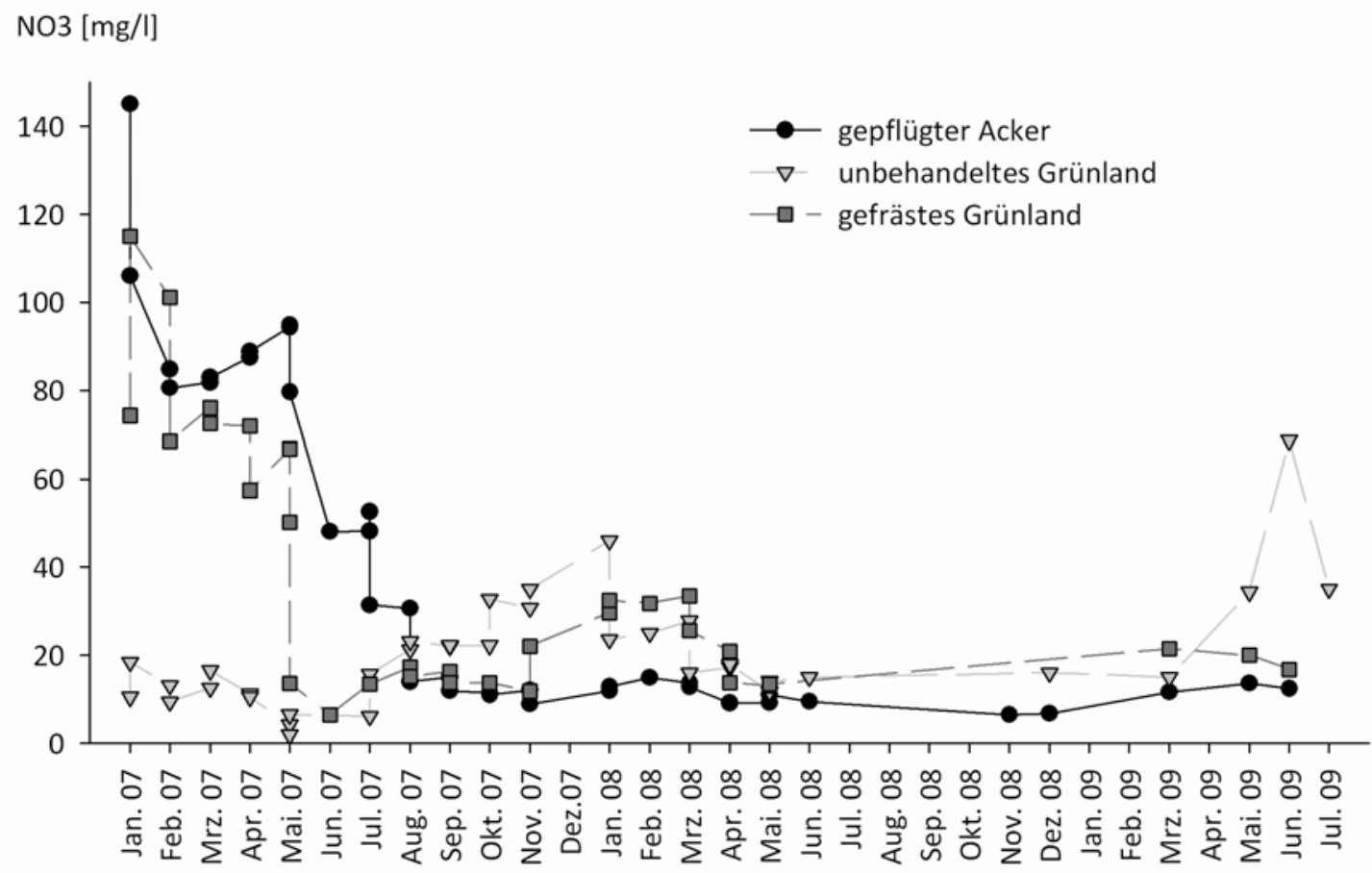

Abb. 4.3: Entwicklung des Austrages an Nitrat mit dem Sickerwasser nach der mechanischen Bearbeitung von Acker und Grünland im Nov. 2006 im Vergleich zu einer unbearbeiteten Grünfläche

Der durchschnittliche Nitrataustrag über den gemessen Zeitraum betrug $42,1 \mathrm{mg} / \mathrm{l}$. Unter dem unbehandelten Grünland konnte über den gesamten betrachteten Zeitraum ein gleichbleibend geringer Nitrataustrag mit dem Sickerwasser von durchschnittlich $16,4 \mathrm{mg} / \mathrm{l}$ gemessen werden. Eine Bodenbearbeitung des Grünlandes, in diesem Fall das Fräsen der Fläche, führte zu einem starken Anstieg des Nitrataustrages, welcher erst nach einigen Monaten wieder auf ein geringes Niveau zurückfiel. Nach dem Fräsen des Grünlandes im November 2006 stieg der Nitrataustrag im Februar 2007 auf bis zu $120 \mathrm{mg} / \mathrm{lim}$ Januar und sank erst im Mai 2007 wieder unter den erlaubten Grenzwert von $50 \mathrm{mg} / \mathrm{l}$ ab. Im Durchschnitt lag der über den gesamten Zeitraum von 2 Jahren gemessene Nitratgehalt des Sickerwassers bei 38,2 mg/l. 


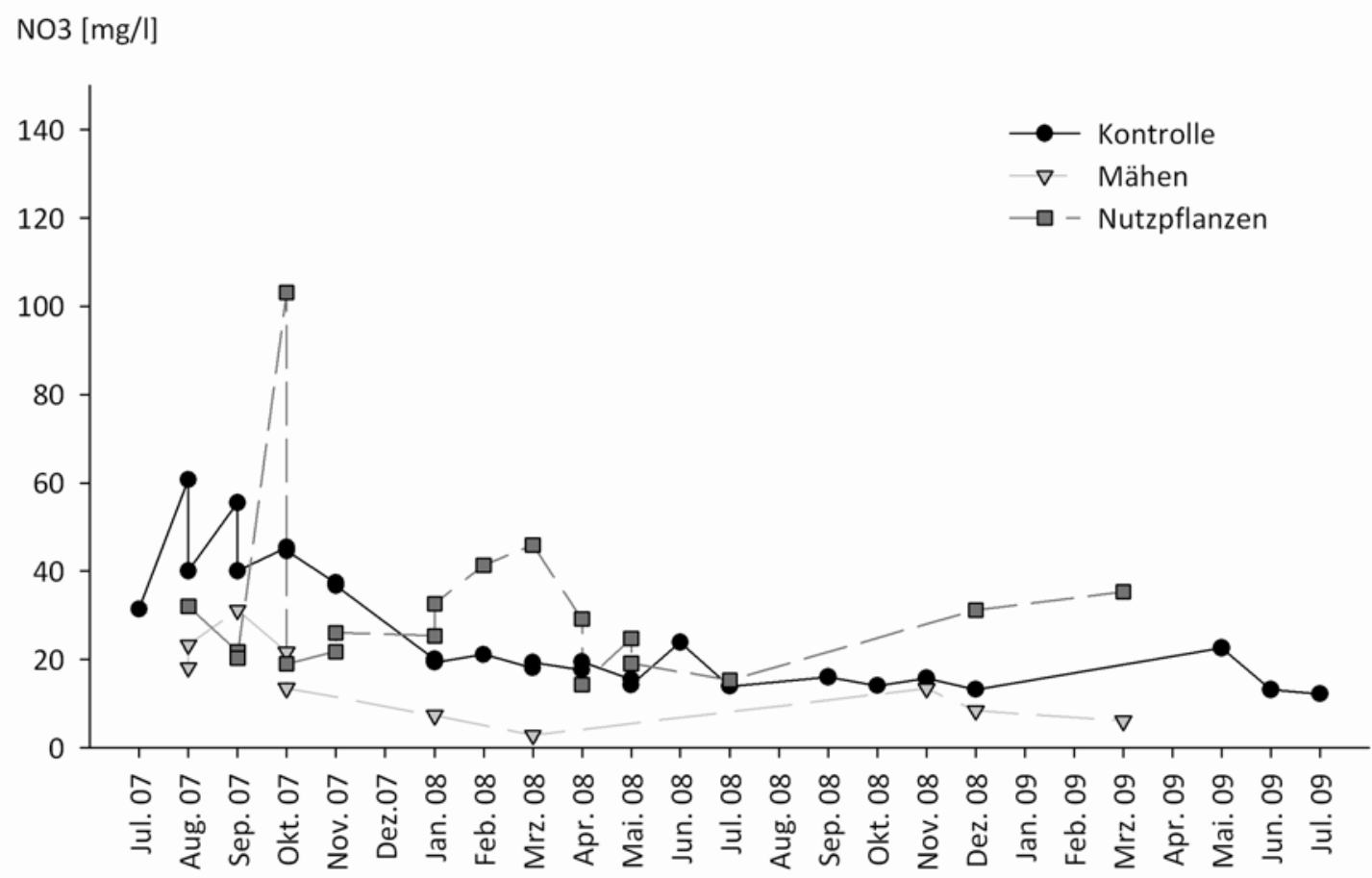

Abb. 4.4: Nitrataustrag mit dem Sickerwasser zwischen den im März 2007 gepflanzten Weidenstecklingen in Abhängigkeit von der Begleitwuchsregulierung (Kontrolle; Mähen; Nutzpflanzeneinsaat)

Auch unter dem Anbau von Weide "Turbo" (Salix viminalis) auf der ehemaligen Ackerfläche war der Nitrataustrag zumeist unterhalb des erlaubten Nitratwertes im Grundwasser von $50 \mathrm{mg} / \mathrm{I}$ (Abb. 4.4). Es gab zwar ein paar Schwankungen im Nitratgehalt abhängig von der angewendeten Begleitwuchsregulierung, aber insgesamt wurde mit allen drei Methoden ein ausreichend geringer Nitratgehalt erreicht. Auf der Kontrollfläche zeigten sich im August und September 2007 Schwankungen im Nitratgehalt, welche sich in Austrägen bis 60 mg/l Nitrat mit dem Sickerwasser wiederspiegelten, aber für den restliche Betrachtungszeitraum gleichbleibend gering bei etwa $20 \mathrm{mg} / \mathrm{l}$ Nitrat blieben. Auf den Flächen mit Nutzpflanzeneinsaat ergab sich im März 2008 ein etwas erhöhter Nitrataustrag, der aber unter dem Grenzwert von $50 \mathrm{mg} / \mathrm{I}$ blieb. Mit dem Abmähen der Vegetation zwischen den Pflanzreihen wurden die geringsten Schwankungen und der durchschnittlich geringste Nitrataustrag mit 15,5 mg/l erreicht. Die Einsaat der Nutzpflanzen und das somit vermehrte Aufkommen von Klee und Buchweizen führten zu einem durchschnittlichen Nitrataustrag von $26,1 \mathrm{mg} / \mathrm{l}$. Der durchschnittliche 
Austrag von Nitrat lag auf der Kontrollfläche ohne durchgeführte Maßnahmen der Begleitwuchsregulierung mit 27,7 mg/I nur unwesentlich höher.

\subsubsection{Bodensaugspannung}

Die Tensiometerwerte von Juli 2007 bis Dezember 2008 (Abb. 4.5) zeigten eine durchschnittliche Bodensaugspannung von $-116 \mathrm{hPa}$ in $10 \mathrm{~cm}$ Bodentiefe, $-120 \mathrm{hPa}$ in $30 \mathrm{~cm}$ Bodentiefe und $-152 \mathrm{hPa}$ in $70 \mathrm{~cm}$ Bodentiefe. Die Unterschiede der Bodensaugspannung auf dem Acker- und Grünland waren auf der Versuchsfläche in Gütersloh nur geringfügig.

Auf der ehemaligen Ackerfläche wurden Bodensaugspannungen von durchschnittlich $-108 \mathrm{hPa}$ in $10 \mathrm{~cm},-128 \mathrm{hPa}$ in $30 \mathrm{~cm}$ und $-158 \mathrm{hPa}$ in $70 \mathrm{~cm}$ Bodentiefe gemessen. Auf der Grünfläche lagen die durchschnittlichen Messwerte der Bodensaugspannung bei $-123 \mathrm{hPa}$ in $10 \mathrm{~cm},-113 \mathrm{hPa}$ in $30 \mathrm{~cm}$ und bei $-146 \mathrm{hPa}$ in $70 \mathrm{~cm}$ Bodentiefe. Die unterschiedlichen Maßnahmen zur Begleitwuchsregulierung wirkten sich im Vergleich zur Kontrollvariante unterschiedlich stark auf die Saugspannung im Boden aus. Auf der Ackerfläche mit der Nutzpflanzeneinsaat zeigte sich eine deutlich erhöhte Bodensaugspannung im Vergleich zu den anderen Varianten. In $70 \mathrm{~cm}$ Bodentiefe lag die durchschnittliche Bodensaugspannung bei -189 hPa. Dieser erhöhte Durchschnittswert wird durch den hohen Ausschlag Ende Juli 2008 verursacht, als die Saugspannung bei ungefähr $-800 \mathrm{hPa}$ lag. Auch die anderen Varianten zeigten erhöhte Saugspannungen im Zeitraum Mai, Juni, Juli 2008, die auf der Grünfläche mit Nutzpflanzeneinsaat bei etwa $-350 \mathrm{hPa}$, den gemähten Flächen bei etwa $-450 \mathrm{hPa}$ und der Kontrollfläche bei nur etwa -300 hPa lagen. 

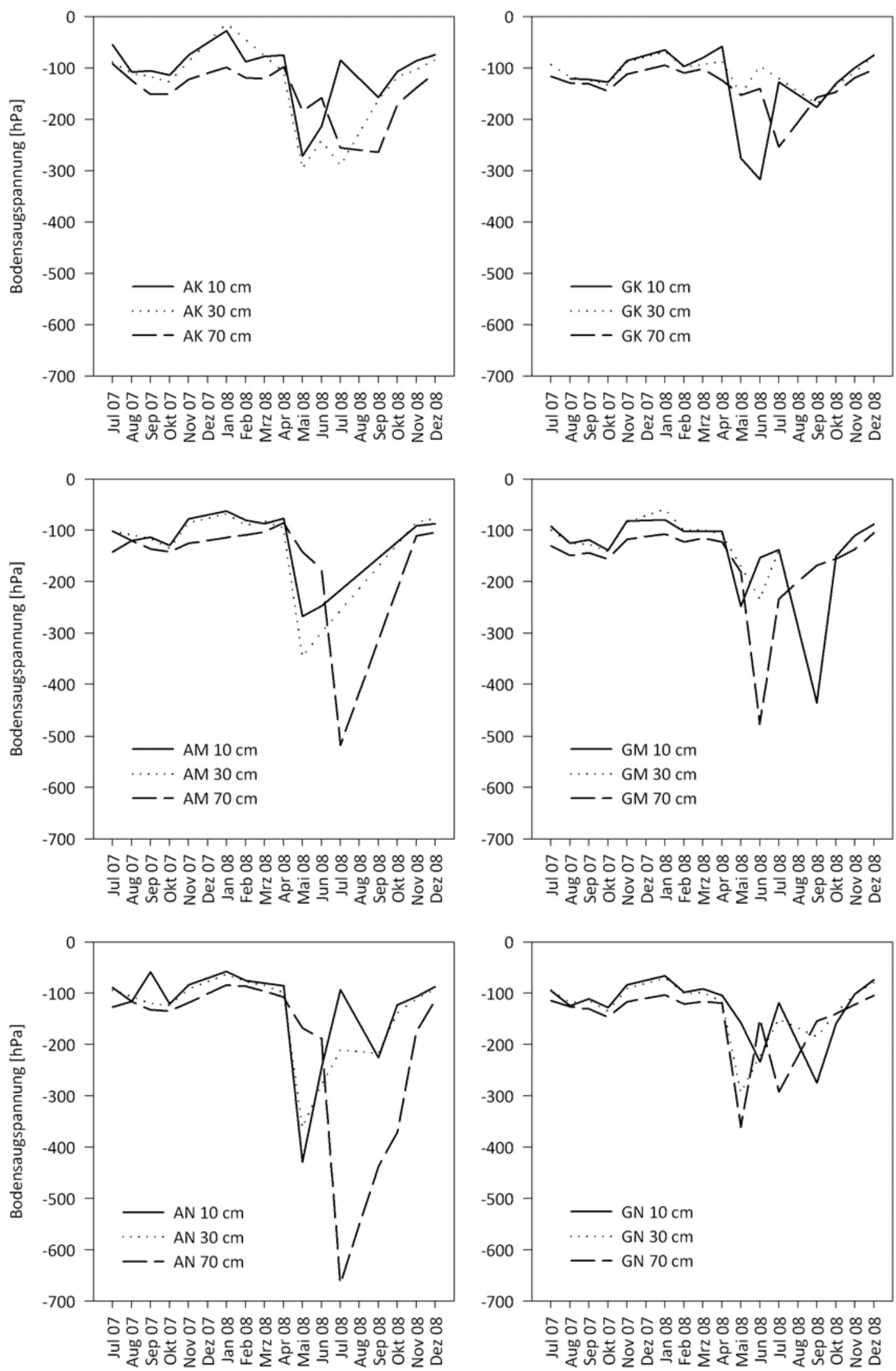

Abb. 4.5: Bodensaugspannung auf der Acker- (A) und Grünlandfläche (G) in den Bodentiefen (10, 30 und $70 \mathrm{~cm}$ ) in Abhängigkeit von der Begleitwuchsregulierung (K: Kontrolle; M: Mähen; N: Nutzpflanzeneinsaat) auf der Versuchsfläche Sudheide in Gütersloh 
Diese Peaks in den Tensiometermesswerten können durch die erhöhten Temperaturen in diesen Monaten und die zudem etwas verringerten Niederschläge im Juli 2008 erklärt werden. Abb. 4.6 verdeutlicht den Zusammenhang zwischen dem Temperaturverlauf und der durchschnittlichen Bodensaugspannung auf der Fläche. Die Diskrepanz zwischen der Saugspannung und der Lufttemperatur von Juli-Oktober 2007 können durch die sehr hohen Niederschläge in diesem Zeitraum erklärt werden, welche den Wasserverlust durch die erhöhten Sommertemperaturen ausgleichen konnten.

Die höhere maximale Saugspannung auf der gemähten Fläche im Vergleich zur Kontrollfläche isł durch die fehlende bzw. reduzierte Vegetationsdecke zu erklären. Insbesondere auf der Ackerfläche wurde durch das Mähen der blanke Boden freigelegt, da die dort vorkommende Vegetation eher Einzelhalmweise stand statt in Horsten. Die Vegetationsdecke diente dem Boden als Verdunstungsschutz, durch welche die Verdunstung der Bodenfeuchtigkeit und somit die Austrocknung der Bodendecke niedriger gehalten wurde als ohne schützende Bodenvegetation.

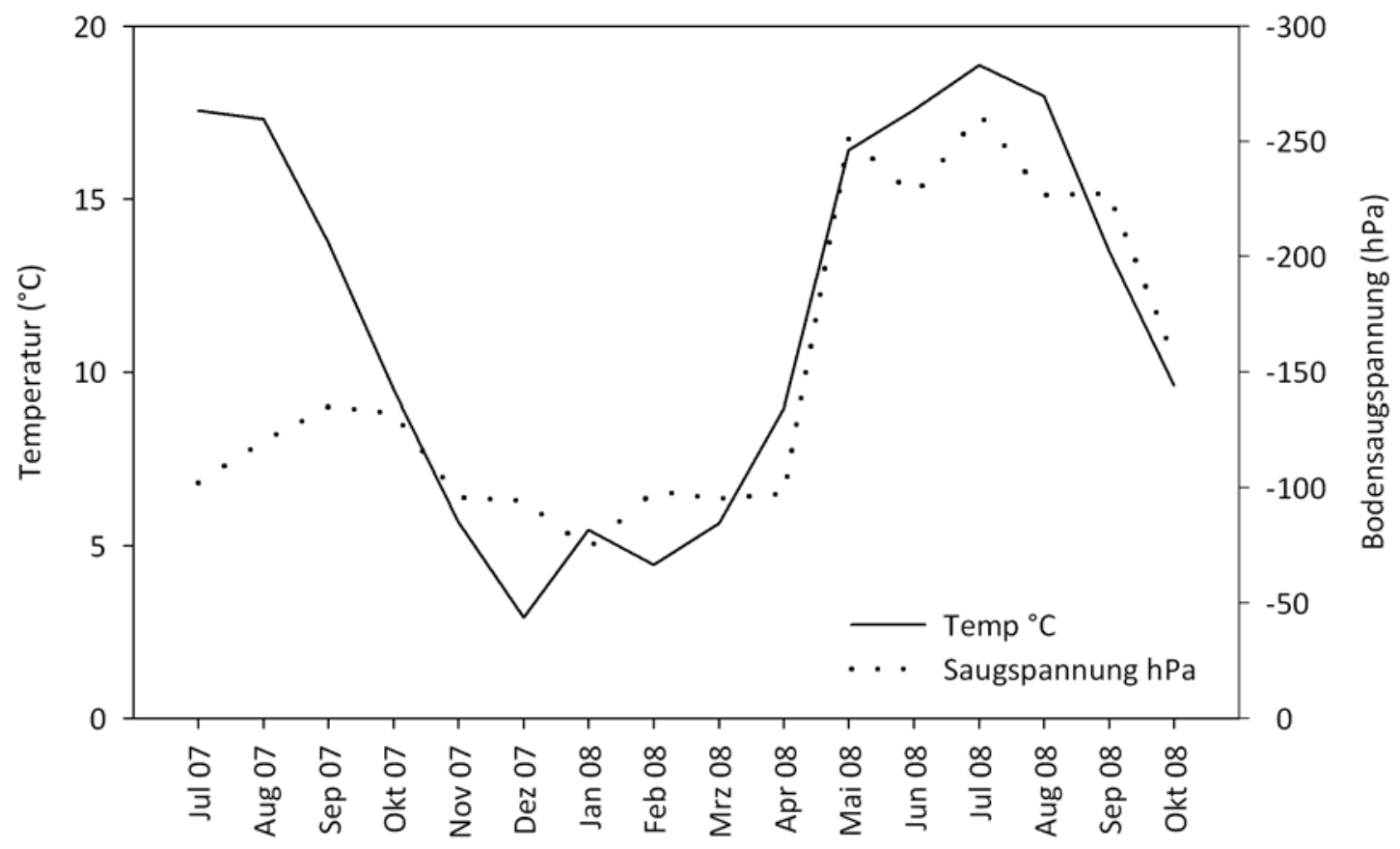

Abb. 4.6: Verlauf der Temperatur und Bodensaugspannung in den Jahren 2007 und 2008 auf der Versuchsfläche Sudheide in Gütersloh 


\subsubsection{Vegetationsaufnahme}

Auf der Grünlandfläche entstand im Anlagejahr 2007 durch das Aufkommen unerwünschter Begleitvegetation ein wesentlich höherer Konkurrenzdruck für die Gehölze als auf der Ackerfläche. Die auf der Grünlandfläche von der Begleitvegetation gebildete Biomasse war ohne Regulierungsmaßnahmen fast doppelt so hoch wie auf der Ackerfläche. Auch die Artenzusammensetzung der Vegetation gestaltete sich auf den unterschiedlich vorgenutzten Flächen differenziert voneinander (Tab. 4.2).

Die natürliche Begleitvegetation produzierte 2007 auf den Kontrollflächen der Ackerfläche signifikant weniger Biomasse als auf der Grünlandfläche. Auf der Ackerfläche wurde ca. $200 \mathrm{~g} / \mathrm{m}^{2}$ Biomasse von der Begleitvegetation gebildet, während diese auf der Grünlandfläche etwa $500 \mathrm{~g} / \mathrm{m}^{2}$ Biomasse produzierte Abb. 4.7).

Tab. 4.2: Hauptpflanzenarten der Begleitvegetation auf der Versuchsfläche Sudheide in Gütersloh

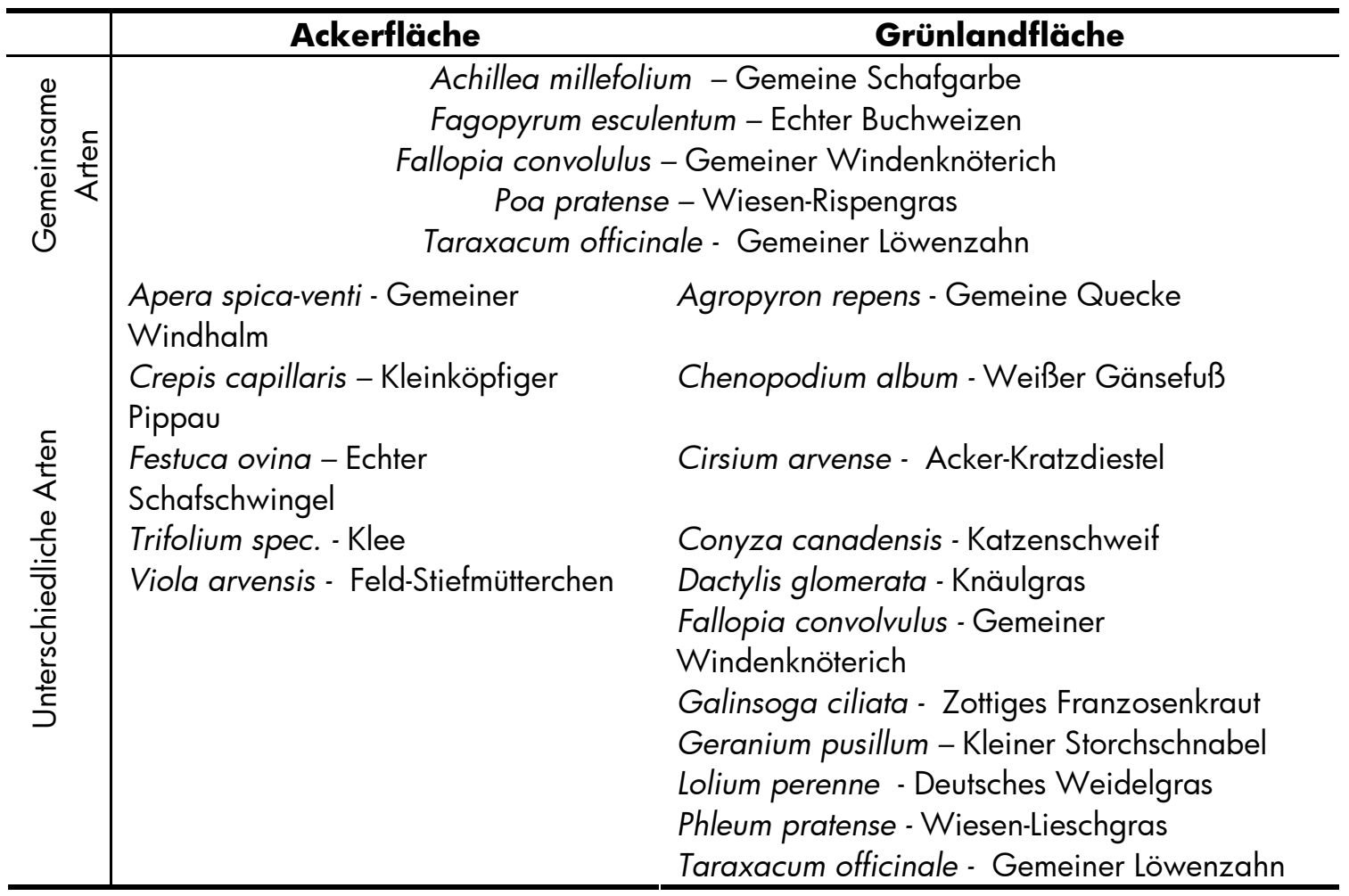


Das Mähen der Flächen verringerte die oberirdische Biomasse der Begleitvegetation im Anlagejahr signifikant. Auf der Ackerfläche betrug die Biomasse der Begleitvegetation einen Monat nach dem Mähvorgang etwa $125 \mathrm{~g} / \mathrm{m}^{2}$. Auf der Grünlandfläche reduzierte das Mähen die Biomasse der Begleitvegetation auf $170 \mathrm{~g} / \mathrm{m}^{2}$.

Auch die eingesäten Nutzpflanzen haben die Biomassebildung der natürlichen Begleitvegetation im Jahr der Kulturbegründung sowohl auf der Acker- als auch auf der Grünlandfläche signifikant reduziert. Die Intensität des Aufkommens der Einsaat war dabei auf der Ackerfläche wesentlich höher als auf der Grünlandfläche. Auf der Ackerfläche produzierten die Kleesorten ca. $370 \mathrm{~g} / \mathrm{m}^{2}$ und der Buchweizen $80 \mathrm{~g} / \mathrm{m}^{2}$ Biomasse. Auf der Grünlandfläche ging nur die Buchweizensaat auf und bildete ebenfalls ca. $80 \mathrm{~g} / \mathrm{m}^{2}$ oberirdische Biomasse. Die Kleesaat war auf der Grünlandfläche nicht erfolgreich.

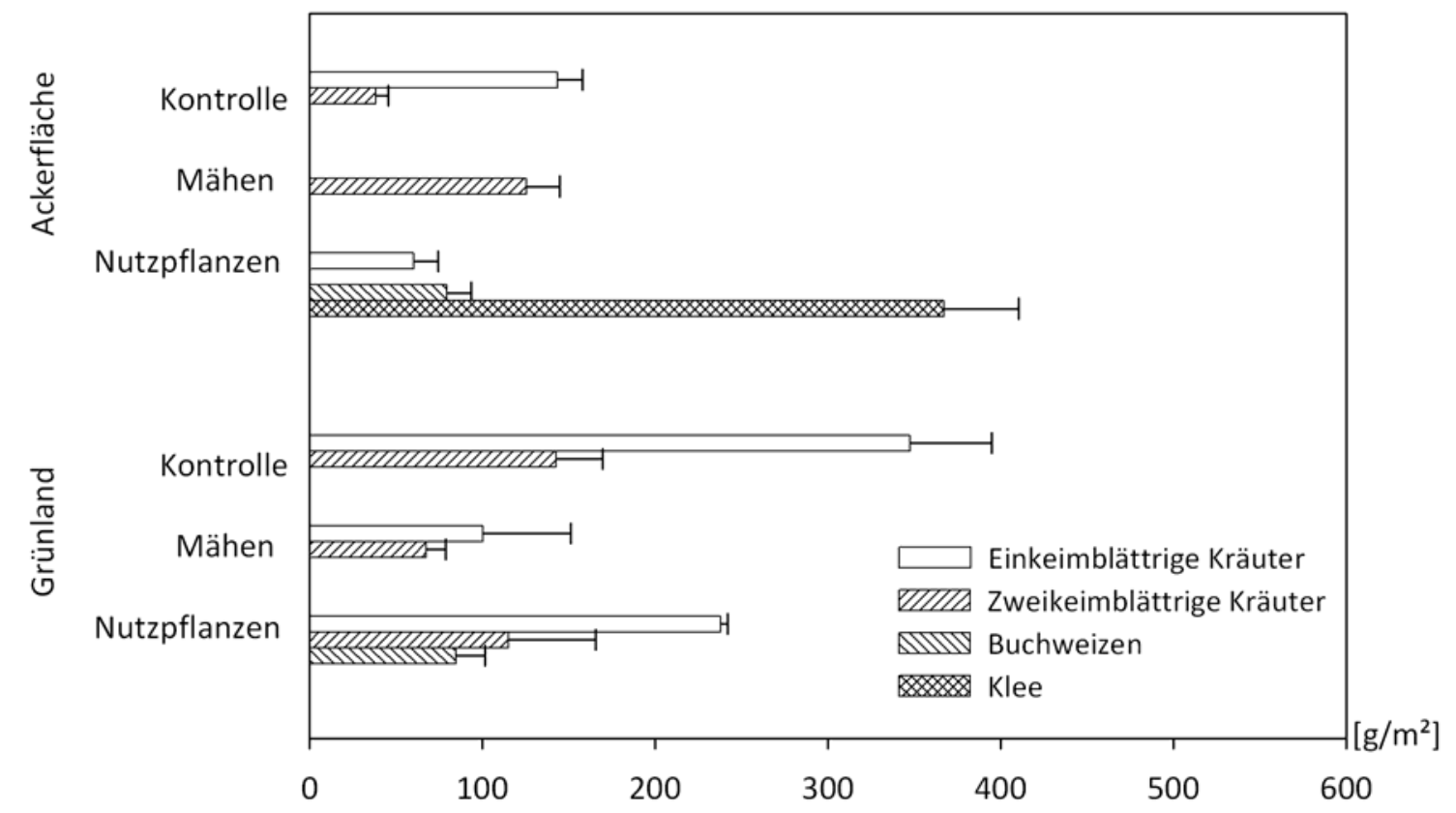

Abb. 4.7: Trockenmasse $\left(\mathrm{g} / \mathrm{m}^{2}\right)$ der natürlichen Begleitvegetation (ein-, zweikeimblättrige Kräuter) und der eingesäten Nutzpflanzen (Buchweizen, Klee) in der ersten Vegetationsperiode (Juli 2007) in Abhängigkeit von der Begleitwuchsregulierung auf der Acker- und Grünlandfläche in Gütersloh ( \pm Standardfehler) 
Nur wenige Arten dominierten die Begleitvegetation auf der im Herbst vor der Kulturbegründung gepflügten Ackerfläche. Auf der Kontrollfläche bildete der Gemeine Windhalm (Apera spica-venti) hauptsächlich die Biomasse, während auf der gemähten Ackerfläche der Gemeine Windenknöterich (Fallopia convolvulus) und das FeldStiefmütterchen (Viola arvensis) die meiste Biomasse bildeten. Der ausgesäte Klee und Buchweizen verdrängte den hochgewachsenen Windhalm auf der Ackerfläche. Die Nutzpflanzen führten ihrerseits zu einer sehr starken Biomassebildung, welche die auf der Kontrollfläche bei weitem überstieg.

Auf der Grünlandfläche war die Begleitvegetation deutlich artenreicher als auf dem Acker. Auf der Kontrollfläche waren vor allem Gräser wie das Wiesen-Rispengras (Poa pratense) Knäulgras (Dactylis glomerata), Deutsches Weidelgras (Lolium perenne) und Wiesen-Lieschgras (Phleum pratense) zu finden. Aber auch Weißer Gänsefuß (Chenopodium album) und Gemeiner Windenknöterich (Fallopia convolvulus) machten einen Großteil der Biomasse aus. Das Mähen der Grünfläche reduzierte die Artenvielfalt und das Deutsche Weidelgras bildete den Hauptteil der Biomasse auf der gemähten Teilfläche. Von den eingesäten Nutzpflanzen ging auf der Grünfläche einzig der Buchweizen auf und verdrängte das Knäulgras und den Gemeinen Windenknöterich.

Die Vegetationsaufnahme in 2008 zeigte auf der Ackerfläche im Vergleich zum Vorjahr ein deutlich vermehrtes Aufkommen der einkeimblättrigen und zweikeimblättrigen Kräuter. Auf der Ackerfläche unterschied sich das Biomasseaufkommen der verschieden gepflegten Teilflächen signifikant, während das Biomasseaufkommen auf der Grünfläche relativ ausgeglichen war (Abb. 4.8). Auf der Kontrollfläche ohne Regulierungsmaßnahmen bildete die Begleitvegetation etwa $310 \mathrm{~g} / \mathrm{m}^{2}$ Biomasse. Das Mähen der Ackerfläche reduzierte die Biomasse der natürlichen Begleitvegetation $150 \mathrm{~g} / \mathrm{m}^{2}$, insbesondere der Anteil einkeimblättriger Kräuter wurde im Vergleich zur Kontrollfläche verringert. Auf der Ackerfläche mit Nutzpflanzeneinsaat dominierte im zweiten Jahr der eingesäte Klee die 
Biomassebildung mit $360 \mathrm{~g} / \mathrm{m}^{2}$. Die von der natürlichen Begleitvegetation gebildete Biomasse wurde auf etwa $230 \mathrm{~g} / \mathrm{m}^{2}$ reduziert.

Auf der Grünlandfläche bildete die natürliche Begleitvegetation im zweiten Standjahr zwischen 220 und $280 \mathrm{~g} / \mathrm{m}^{2}$ Trockenbiomasse. Die eingesäten Nutzpflanzen waren mit $2 \mathrm{~g} / \mathrm{m}^{2}$ Buchweizen und $10 \mathrm{~g} / \mathrm{m}^{2}$ Klee nur noch selten zu finden.

Die Kontrollfläche auf dem ehemaligen Acker wurde im zweiten Jahr mit einem Deckungsgrad von 66 \% deutlich vom Gemeinen Windhalm dominiert. Des Weiteren waren Katzenschweif und Löwenzahn mit jeweils fasł $20 \%$ vertreten. Durch das Mähen der Ackerfläche wurde der Deckungsgrad des Windhalmes auf $14 \%$ verringert. Katzenschweif und Löwenzahn waren im gleichen Maße wie auf der Kontrollfläche vertreten. Auf der mit Nutzpflanzen eingesäten Ackerfläche dominierte der eingesäte Klee die Bodenvegetation mit einem Deckungsgrad von 66 \%, während der Echte Buchweizen nicht mehr zu finden war. Der Deckungsgrad der natürlichen Begleitvegetation wie des Gemeinen Windhalmes war mit $36 \%$ geringer als auf der Kontrollfläche, ebenso wie der Deckungsgrad des Katzenschweifes mit $11 \%$ und des Gemeinen Löwenzahns mit 4 \%. Die Acker-Kratzdiestel, welche auf der Kontrollfläche kaum vorkam, erreichte dagegen einen Deckungsgrad von $20 \%$.

Die Grünlandfläche ohne Pflegemaßnahmen wurde in der zweiten Vegetationsperiode von dem Echten Schafschwingel mit einem Deckungsgrad von $42 \%$ und der Gemeinen Schafgarbe mit 18 \% dominiert. Auf der gemähten Grünfläche dominierte ebenfalls der Echte Schafschwingel mit allerdings 62 \%. Der Kleinköpfige Pippau und Gemeine Löwenzahn kamen mit einem Deckungsgrad von etwa $15 \%$ auf der gemähten Grünfläche vor. Auf der mit Nutzpflanzen eingesäten Grünfläche dominierten insbesondere der Echte Schafschwingel mit 48 \%igem Deckungsgrad und das Wiesen-Rispengras mit 18 \% Bodendeckung. Die Nutzpflanzen erreichten in der zweiten Vegetationsperiode nur noch sehr geringe Deckungsgrade von 1-2 \%. 


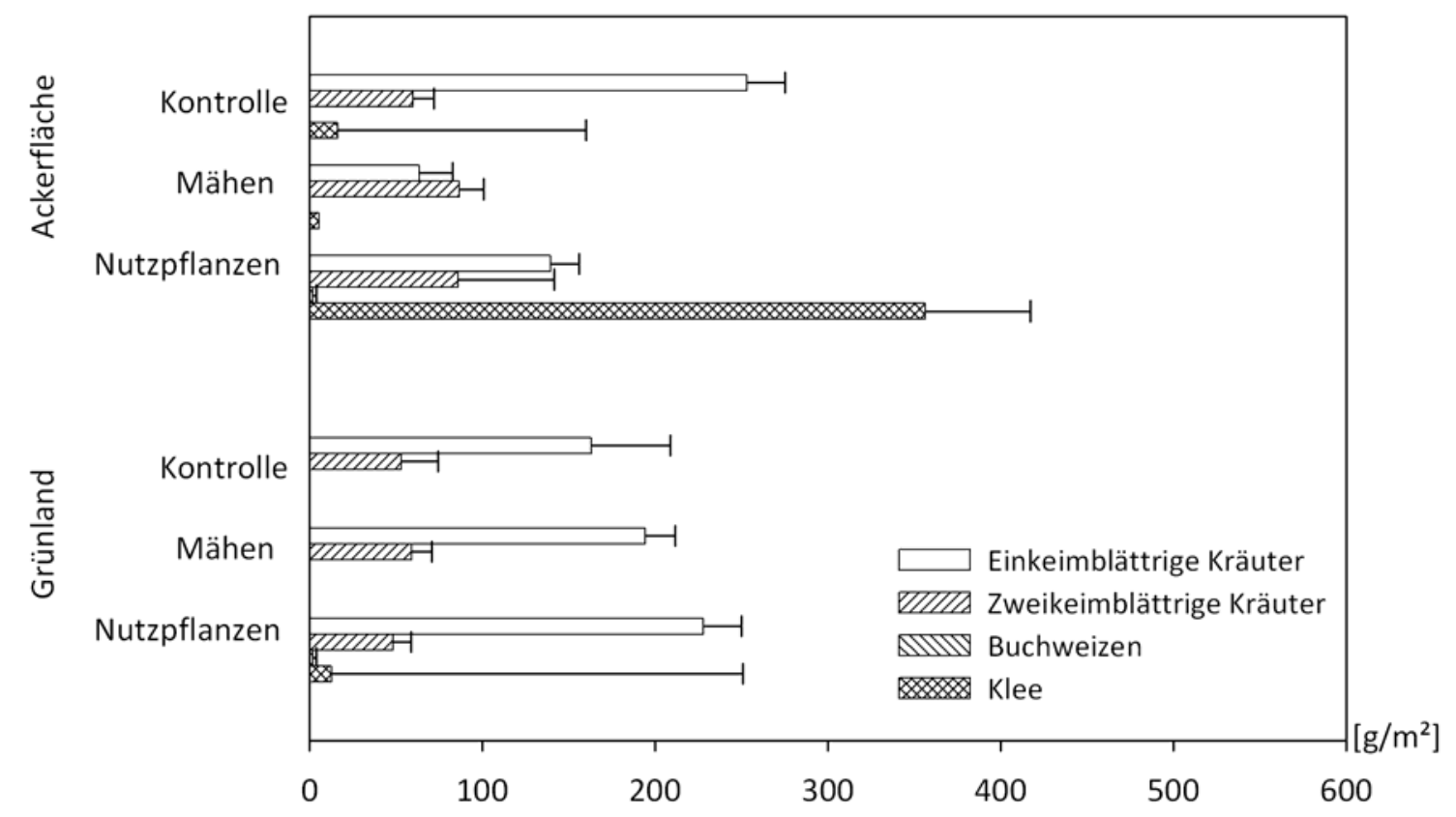

Abb. 4.8: Trockenmasse $\left(g / \mathrm{m}^{2}\right)$ der natürlichen Begleitvegetation (ein-, zweikeimblättrige Kräuter) und der eingesäten Nutzpflanzen (Buchweizen, Klee) in der zweiten Vegetationsperiode (Juli 2008) in Abhängigkeit von der Begleitwuchsregulierung auf der Acker- und Grünlandfläche in Gütersloh ( \pm Standardfehler)

\subsubsection{Anwuchs- / Vitalitätsbewertung}

\subsubsection{Flächenvornutzung}

Die Flächenvornutzung hatte auf der Versuchsfläche Sudheide in Gütersloh einen signifikanten Einfluss auf den Anwuchs der Gehölze. Der Anwuchserfolg der jungen Pflanzen war auf der Ackerfläche signifikant höher als auf dem Grünland. Der Anwuchserfolg von Aspe, Robinie Weide und Winterlinde lag auf der Kontrollfläche des Ackers bei etwa 100 \% (Tab. 4.3). Eberesche und "Max 4" wiesen mit rund $90 \%$, Bergahorn und "NE 42" mit $80 \%$ geringere Anwuchsraten auf. Der geringste Anwuchs wurde mit knapp $50 \%$ von der Schwarzerle erbracht. Auf den Kontrollflächen des Grünlandes wuchsen etwa $80 \%$ der Aspen und $90 \%$ der Robinien und Weiden an. Der Anwuchserfolg der Pappelstecklinge war mit etwa $70 \%$ bei "Max 4" und knapp $30 \%$ bei "NE 42" signifikant geringer als bei den anderen Baumarten. 
Tab. 4.3: Anwuchserfolg (\%) der Gehölze auf der Acker- und Grünlandfläche in der ersten Vegetationsperiode in Abhängigkeit von der Begleitwuchsregulierung ( \pm Standardfehler; * signifikante Mittelwert-Differenz zur Kontrolle mit $p \leq 0,05$ )

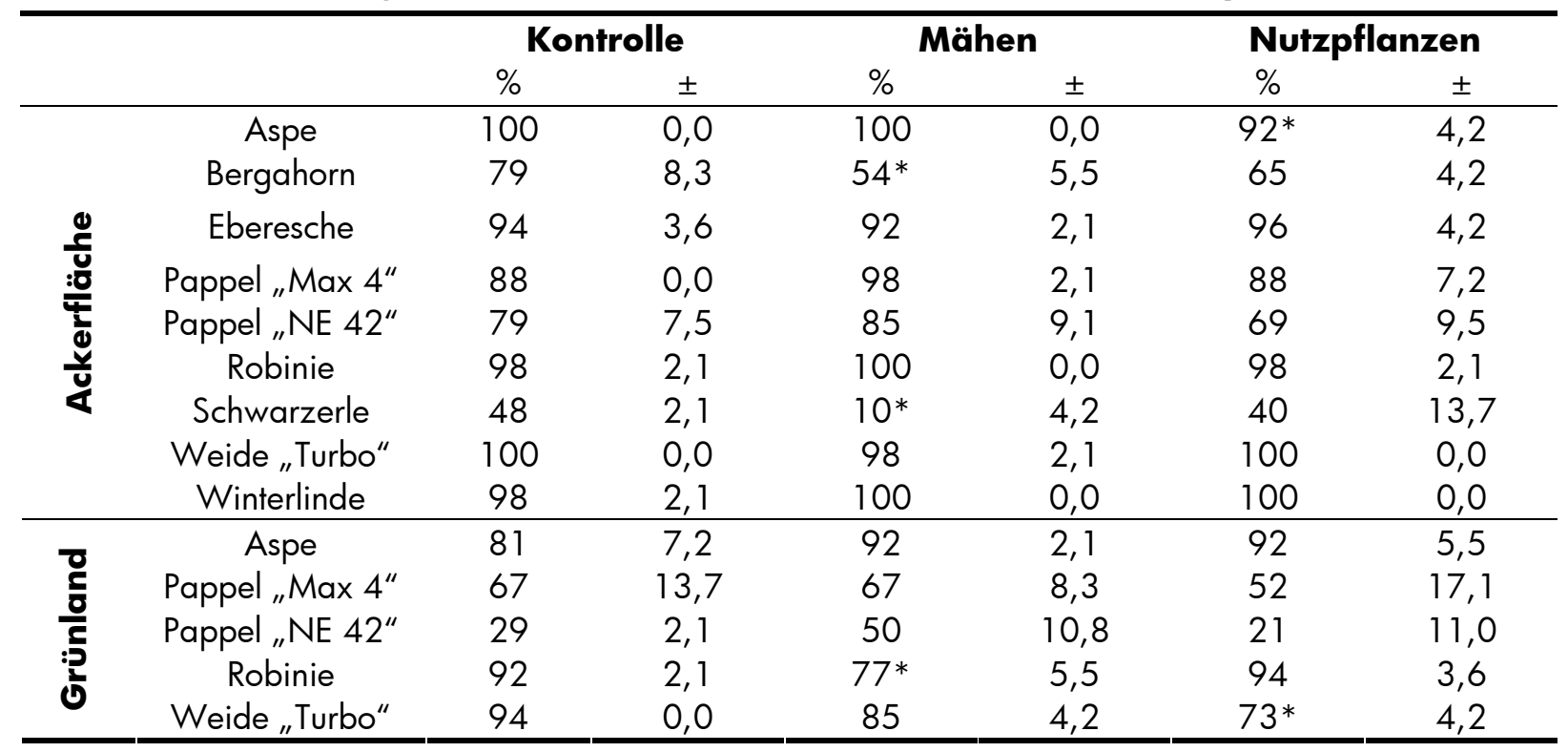

\subsubsection{Begleitwuchsregulierung}

Die Maßnahmen zur Begleitwuchsregulierung (Mähen; Nutzpflanzen) hatten nur in Einzelfällen einen signifikanten Effekt auf die Anwuchsraten der gepflanzten Gehölze (Tab. 4.3). Auf der Ackerfläche war der Anwuchs der Aspe auf der Teilfläche mit eingesäten Nutzpflanzen signifikant niedriger als auf der Kontrollfläche ohne Begleitwuchsregulierung. Auf der gemähten Ackerfläche war der Anwuchserfolg des Bergahorns und der Schwarzerle im Vergleich zur Kontrollfläche signifikant geringer. Auf der Grünlandfläche wuchsen signifikant weniger Robinien auf der gemähten Teiffläche als auf der Kontrollfläche an. Auf der mit Nutzpflanzen eingesäten Grünfläche war der Anteil der ausgetriebenen Weidenstecklinge signifikant geringer als auf der Kontrollfläche.

In der zweiten Vegetationsperiode war die Überlebensrate der Gehölze (Tab. 4.4) geringer als in der ersten Vegetationsperiode. Von durchschnittlich $80 \%$ sank die Anzahl der noch lebenden Pflanzen auf etwa 70 \%. Die Überlebensrate von der ersten zur zweiten Vegetationsperiode sank unabhängig von der Begleitwuchsregulierung um etwa $10 \%$. Zwischen den Baumarten gab es große Unterschiede in der Ausfallrate. 
Bei den Aspen und Robinien überlebten insgesamt nur $70 \%$ der im Vorjahr angewachsenen Pflanzen auch das zweite Jahr.

Bei Betrachtung der ersten zwei Jahre zeigten außer Robinie und Schwarzerle alle Baumarten mit mindestens $80 \%$ eine befriedigende Überlebensrate auf der Kontrollfläche der Ackerfläche. Auf der Grünfläche konnte nur die Weide mit etwa $90 \%$ eine befriedigende Überlebensrate aufweisen. Die restlichen Baumarten hatten auf der Grünlandfläche Überlebensraten von nur 20 \% (Pappel "NE 42") bis maximal $70 \%$ (Aspe).

Tab. 4.4: Überlebensrate (\%) der Gehölze auf der Acker- und Grünlandfläche nach der zweiten Vegetationsperiode in Abhängigkeit von der Begleitwuchsregulierung ( \pm Standardfehler; * signifikante Mittelwert-Differenz zur Kontrolle mit $\mathbf{p} \leq \mathbf{0 , 0 5}$ )

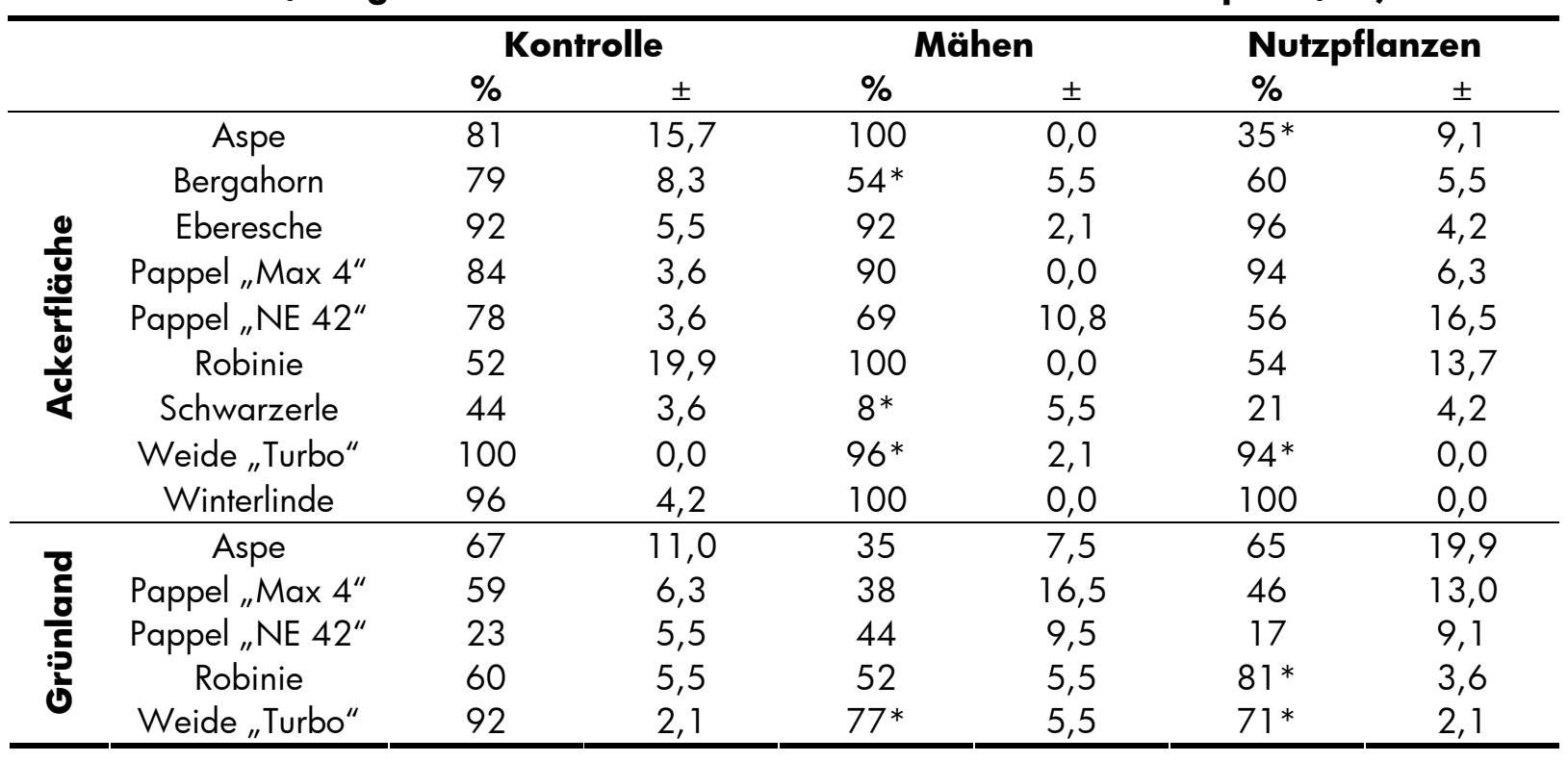

Auch nach der zweiten Vegetationsperiode war der Einfluss der Begleitwuchsregulierung auf die Überlebensrate der Gehölze gering. Wie nach der ersten Vegetationsperiode war der Anteil der noch lebenden Aspen auf der mit Nutzpflanzen eingesäten Teilfläche und die Überlebensrate des Bergahorns und der Schwarzerle auf der gemähten Ackerfläche signifikant geringer als auf der Kontrollfläche. In der zweiten Vegetationsperiode waren auf der gemähten und der mit Nutzpflanzen eingesäten Ackerfläche einige Weiden abgestorben, so dass die Überlebensrate auf diesen Flächen signifikant geringer war als auf der Kontrollfläche. 
Die Sterbensrate der Robinie war auf der Grünlandfläche nach dem ersten Standjahr relativ hoch. Der geringste Verlust war auf der mit Nutzpflanzen eingesäten Grünfläche zu verzeichnen, so dass die Überlebensrate auf dieser Teilfläche signifikant höher als auf der Kontrollfläche war. Der Weidensteckling "Turbo" hatte wie auf der Ackerfläche eine signifikant höhere Überlebensrate auf der Kontrollfläche als auf den Flächen mit durchgeführter Begleitwuchsregulierung.

\subsubsection{Bodenhilfsstoffe}

Die verwendeten Hilfsmittel (Hydrogel, Mykorrhiza) führten nur in Einzelfällen zu signifikanten Auswirkungen auf die Anwuchsrate (Tab. 4.5) und Vitalität der Gehölze (Tab. 4.6; Tab. 4.7). Es war keine für alle Baumarten gültige Wirkung der Hilfsstoffe auf Anwuchs- und Zuwachsraten auszumachen.

Tab. 4.5: Anwuchs- und Überlebensraten der Gehölze auf der Acker- und Grünlandfläche in der ersten ('07) und zweiten ('08) Vegetationsperiode in Abhängigkeit von den verwendeten Bodenhilfsstoffen (*signifikanter Unterschied zur Kontrolle mit $p \leq 0,05$ )

\begin{tabular}{|c|c|c|c|c|c|c|c|c|c|}
\hline & & \multicolumn{2}{|c|}{ Kontrolle } & \multicolumn{2}{|c|}{ Hydrogel } & \multicolumn{2}{|c|}{ Mykorrhiza } & \multicolumn{2}{|c|}{$\begin{array}{l}\text { Hydrogel + } \\
\text { Mykorrhiza }\end{array}$} \\
\hline & & 07 & 08 & 07 & 08 & ’07 & 08 & 07 & '08 \\
\hline \multirow{9}{*}{ 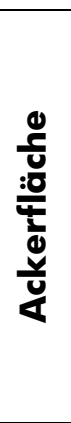 } & Aspe & 100 & 81 & $94^{*}$ & $50 *$ & 94 & 100 & $94^{*}$ & 94 \\
\hline & Bergahorn & 79 & 79 & 94 & 81 & 69 & 69 & 94 & 94 \\
\hline & Eberesche & 94 & 92 & 100 & 100 & $38^{*}$ & $38 *$ & 81 & 81 \\
\hline & Pappel "Max 4" & 88 & 84 & 88 & 84 & 94 & 90 & 88 & 80 \\
\hline & Pappel "NE 42" & 79 & 78 & 75 & $56^{*}$ & 94 & 69 & 69 & 61 \\
\hline & Robinie & 98 & 52 & 100 & 75 & 44 & 40 & 100 & 56 \\
\hline & Schwarzerle & 48 & 44 & $75^{*}$ & $69 *$ & 13 & $8^{*}$ & 63 & 44 \\
\hline & Weide "Turbo" & 100 & 100 & 100 & 100 & 94 & $90^{*}$ & 100 & 100 \\
\hline & Winterlinde & 98 & 96 & 100 & 100 & 100 & 100 & 100 & 100 \\
\hline \multirow{5}{*}{ 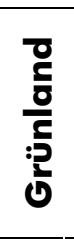 } & Aspe & 81 & 67 & 88 & 75 & 69 & 64 & 81 & 56 \\
\hline & Pappel "Max 4" & 67 & 59 & $31^{*}$ & 30 & 56 & 50 & 44 & 40 \\
\hline & Pappel "NE 42" & 29 & 23 & $0^{*}$ & $0 *$ & $19 *$ & 19 & 50 & $50 *$ \\
\hline & Robinie & 92 & 60 & 81 & 75 & 63 & 58 & 88 & $31 *$ \\
\hline & Weide "Turbo" & 94 & 92 & $50 *$ & $45^{*}$ & $75^{*}$ & $75^{*}$ & 88 & 88 \\
\hline
\end{tabular}

Auf der Ackerfläche zeigte sich ein signifikanter Effekt der Hydrogel-Beigabe auf die Anwuchsraten der Gehölze vor allem im zweiten Standjahr. In der zweiten Vegetationsperiode lebten auf der Ackerfläche nur 50 \% der mit Hydrogel gepflanzten 
Aspen, während noch $80 \%$ der Kontrollpflanzen lebten $(p \leq 0,05)$. Auch auf die Überlebensrate des Pappelstecklings "NE 42" hatte die Hydrogel-Applikation einen signifikant negativen Effekt, der die Überlebensrate der Pappelstecklinge im zweiten Jahr deutlich reduzierte $(p \leq 0,005)$. Bei der Robinie war auf der Ackerfläche dagegen eine positive Tendenz des Hydrogels sichtbar. Die Überlebensrate der Schwarzerle war wie im ersten Jahr auch in der zweiten Vegetationsperiode signifikant höher wenn Hydrogel appliziert wurde $(p \leq 0,05)$. Ohne Hilfsstoffe betrug die Anwuchsrate der Schwarzerle $50 \%$, während diese mit Hydrogel 75 \% betrug. Auf der Grünfläche wurden die Anwuchsraten der Pappel- und Weidenstecklinge von der HydrogelBeigabe signifikant beeinflusst. Bei "Max 4" betrug die Überlebensrate mit Hydrogel nur $30 \%$, statt fast $70 \%$ wie bei den Kontrollpflanzen. Bei dem Pappelsteckling "NE 42" kam es mit Hydrogelbeigabe zu einem Totalausfall, der auch im zweiten Standjahr noch zu einem signifikanten Unterschied in der Anwuchsrate wischen Kontrollpflanzen und den mit Hydrogel gepflanzten Stecklingen führte $(p \leq 0,05)$. Die Weidenstecklinge wiesen ohne Hilfsstoffe eine Anwuchsrate von über $90 \%$ auf, während diese mit Hydrogelbeigabe nur $50 \%$ betrug. Und auch bei den Weidenstecklingen blieb der Unterschied zwischen den Kontrollpflanzen und den mit Hydrogel behandelten Pflanzen im zweiten Standjahr signifikant $(p \leq 0,05)$

Die Mykorrhizierung der Wurzeln bei der Pflanzung zeigte in der ersten Vegetationsperiode bei der Eberesche auf der Ackerfläche einen höchst signifikanten negativen Einfluss auf die Anwuchsrate. Die Anwuchsrate der Kontrollpflanzen lag bei über 90 \%, während von den mykorrhizierten Ebereschen nur etwa 40 \% anwuchsen $(p \leq 0,01)$. Dieser signifikante Unterschied blieb auch in der zweiten Vegetationsperiode bestehen. Bei dem Pappelsteckling "Max 4" war auf der Ackerfläche der Effekt der Mykorrhizierung nicht signifikant, aber es zeigte sich eine positive Tendenz der Mykorrhizierung auf das Anwuchsverhalten. In der zweiten Vegetationsperiode zeigte die Mykorrhizierung auf der Ackerfläche einen signifikant negativen Effekt auf die Überlebensrate der Schwarzerle und Weide $(p \leq 0,05)$. Das Anwuchsverhalten der Weidenstecklinge wurde auf der Grünlandfläche bereits im ersten Jahr signifikant negativ beeinflusst $(p \leq 0,05)$; dieser signifikante Unterschied 
zwischen Kontrollpflanzen und mykorrhizierten Pflanzen blieb in der zweiten Vegetationsperiode bestehen.

Die kombinierte Anwendung von Hydrogel und Mykorrhiza zeigte in der ersten Vegetationsperiode auf der Ackerfläche eine signifikant negative Wirkung auf die Anwuchsrate der Aspe $(p \leq 0,05)$. Auf der Grünlandfläche hatte im zweiten Jahr der Pappelsteckling "NE 42" eine signifikant höhere Überlebensrate wenn beide Bodenhilfsstoffe kombiniert angewendet wurden als die Kontrollpflanzen $(p \leq 0,05)$, während die Überlebensrate der Robinie mit Hydrogel und Mykorrhiza signifikant niedriger war als die der Kontrollpflanzen $(p \leq 0,05)$.

Tab. 4.6: Mittlere Vitalität der lebenden Gehölze auf der Acker- und Grünlandfläche in der ersten Vegetationsperiode in Abhängigkeit von den verwendeten Bodenhilfsstoffen (1 vital; 4 fast völlig abgestorben; \pm Standardfehler; ${ }^{*}$ signifikante Mittelwert-Differenz zur Kontrolle mit $p \leq 0,05$ )

\begin{tabular}{|c|c|c|c|c|c|c|c|c|c|}
\hline & & \multicolumn{2}{|c|}{ Kontrolle } & \multicolumn{2}{|c|}{ Hydrogel } & \multicolumn{2}{|c|}{ Mykorrhiza } & \multicolumn{2}{|c|}{$\begin{array}{l}\text { Hydrogel + } \\
\text { Mykorrhiza }\end{array}$} \\
\hline & & $\varnothing$ & \pm & $\varnothing$ & \pm & $\varnothing$ & \pm & $\varnothing$ & \pm \\
\hline \multirow{9}{*}{$\begin{array}{l}\frac{0}{0} \\
\frac{0}{0} \\
\frac{0}{2} \\
\frac{v}{0} \\
\frac{0}{4}\end{array}$} & Aspe & 1,8 & 0,1 & 1,6 & 0,1 & 1,6 & 0,2 & 1,5 & 0,1 \\
\hline & Bergahorn & 2,0 & 0,1 & 1,9 & 0,2 & 2,4 & 0,3 & 2,3 & 0,2 \\
\hline & Eberesche & 1,4 & 0,1 & 1,5 & 0,1 & $1,0 *$ & 0,0 & 1,5 & 0,1 \\
\hline & Pappel „Max 4" & 1,0 & 0,0 & 1,0 & 0,0 & 1,0 & 0,0 & 1,0 & 0,0 \\
\hline & Pappel "NE 42" & 1,0 & 0,0 & 1,0 & 0,0 & 1,0 & 0,0 & 1,0 & 0,0 \\
\hline & Robinie & 1,3 & 0,1 & 1,2 & 0,1 & 1,1 & 0,1 & 1,3 & 0,2 \\
\hline & Schwarzerle & 1,8 & 0,2 & $1,2^{*}$ & 0,1 & 1,3 & 0,2 & 1,3 & 0,2 \\
\hline & Weide "Turbo" & 1,0 & 0,0 & 1,0 & 0,0 & 1,0 & 0,0 & 1,0 & 0,0 \\
\hline & Winterlinde & 2,1 & 0,1 & 1,9 & 0,1 & 2,1 & 0,1 & 2,1 & 0,2 \\
\hline \multirow{5}{*}{ 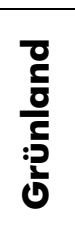 } & Aspe & 2,1 & 0,1 & 1,7 & 0,2 & 2,3 & 0,3 & 1,5 & 0,2 \\
\hline & Pappel "Max 4" & 1,0 & 0,0 & 1,0 & 0,0 & 1,0 & 0,0 & 1,0 & 0,0 \\
\hline & Pappel "NE 42" & 1,0 & 0,0 & $\ldots$ & -- & 1,0 & 0,0 & 1,0 & 0,0 \\
\hline & Robinie & 2,3 & 0,1 & $1,5^{*}$ & 0,1 & 2,5 & 0,2 & $1,5^{*}$ & 0,1 \\
\hline & Weide "Turbo" & 1,0 & 0,0 & 1,0 & 0,0 & 1,0 & 0,0 & 1,0 & 0,0 \\
\hline
\end{tabular}

Die Vitalität der noch lebenden Gehölze in der ersten Vegetationsperiode in Abhängigkeit von den applizierten Hilfsstoffen ist in Tab. 4.6 dargestellt. Die auf der Ackerfläche angewachsenen Schwarzerlen wiesen mit der Hydrogel-Applikation eine signifikant bessere Vitalität als die Kontrollpflanzen auf $(p \leq 0,05)$. Der Anteil der Schwarzerlen in Vitalitätsstufe 1 war mit Hydrogel-Applikation beinahe doppelt so hoch wie bei den Kontrollpflanzen ohne Hydrogel-Beimischung im Bodensubstrat. Auch 
bei der Robinie auf der Grünlandfläche war in der ersten Vegetationsperiode eine signifikant bessere durchschnittliche Vitalität mit Hydrogel als ohne festzustellen $(p \leq 0,001)$.

Die Mykorrhizierung führte in der ersten Vegetationsperiode lediglich bei der Eberesche auf der Ackerfläche zu einem signifikanten Unterschied in der Vitalität im Vergleich zu den Kontrollpflanzen $(p \leq 0,05)$.

Die Vitalität der Robinien auf der Grünlandfläche war in der ersten Vegetationsperiode sowohl mit der alleinigen Hydrogel-Beigabe, als auch mit der kombinierten Anwendung von Hydrogel und Mykorrhiza signifikant besser als die Kontrollpflanzen $(p \leq 0,001)$.

Tab. 4.7: Mittlere Vitalität der lebenden Gehölze auf der Acker- und Grünlandfläche in der zweiten Vegetationsperiode in Abhängigkeit von den verwendeten Bodenhilfsstoffen (1 vital; 4 fast völlig abgestorben; \pm Standardfehler; ${ }^{*}$ signifikante Mittelwert-Differenz zur Kontrolle mit $p \leq 0,05$ )

\begin{tabular}{|c|c|c|c|c|c|c|c|c|c|}
\hline & & \multicolumn{2}{|c|}{ Kontrolle } & \multicolumn{2}{|c|}{ Hydrogel } & \multicolumn{2}{|c|}{ Mykorrhiza } & \multicolumn{2}{|c|}{$\begin{array}{l}\text { Hydrogel + } \\
\text { Mykorrhiza }\end{array}$} \\
\hline & & $\varnothing$ & \pm & $\varnothing$ & \pm & $\varnothing$ & \pm & $\varnothing$ & \pm \\
\hline \multirow{9}{*}{$\begin{array}{l}\frac{0}{0} \\
\frac{0}{0} \\
\frac{0}{0} \\
\frac{v}{0} \\
\frac{0}{4}\end{array}$} & Aspe & 1,3 & 0,1 & $2,1^{*}$ & 0,3 & 1,5 & 0,2 & 1,3 & 0,2 \\
\hline & Bergahorn & 1,4 & 0,1 & 1,5 & 0,1 & 1,5 & 0,2 & 1,7 & 0,2 \\
\hline & Eberesche & 2,9 & 0,1 & 3,0 & 0,0 & 2,8 & 0,2 & 3,1 & 0,1 \\
\hline & Pappel "Max 4" & 1,0 & 0,0 & $1,2 *$ & 0,1 & 1,0 & 0,0 & 1,0 & 0,0 \\
\hline & Pappel "NE 42" & 2,1 & 0,0 & 2,1 & 0,1 & 2,1 & 0,1 & 2,0 & 0,0 \\
\hline & Robinie & 2,4 & 0,2 & 2,8 & 0,4 & 1,7 & 0,3 & 2,2 & 0,3 \\
\hline & Schwarzerle & 2,8 & 0,1 & 2,4 & 0,2 & 2,0 & 0,0 & 2,7 & 0,2 \\
\hline & Weide "Turbo" & 1,5 & 0,1 & 1,7 & 0,2 & 1,5 & 0,2 & 1,4 & 0,2 \\
\hline & Winterlinde & 2,4 & 0,1 & 2,2 & 0,1 & 2,2 & 0,1 & 2,6 & 0,2 \\
\hline \multirow{5}{*}{ 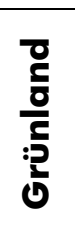 } & Aspe & 1,8 & 0,1 & $1,3^{*}$ & 0,2 & 2,1 & 0,3 & $1,1^{*}$ & 0,1 \\
\hline & Pappel "Max 4" & 1,3 & 0,1 & 2,0 & - & 1,7 & 0,3 & 1,5 & 0,5 \\
\hline & Pappel "NE 42" & 1,0 & 0,0 & - & - & 1,0 & 0,0 & 1,0 & 0,0 \\
\hline & Robinie & 1,8 & 0,1 & 1,8 & 0,3 & $2,5^{*}$ & 0,3 & 1,6 & 0,2 \\
\hline & Weide "Turbo" & 1,7 & 0,1 & 1,9 & 0,2 & $2,2^{*}$ & 0,2 & 1,9 & 0,3 \\
\hline
\end{tabular}

In der zweiten Vegetationsperiode zeigte sich eine signifikant schlechtere durchschnittliche Vitalität der Gehölze im Vergleich zum ersten Standjahr. Die applizierten Hilfsstoffe hatten eine zumeist negative Wirkung auf die durchschnittliche Vitalität der Gehölze (Tab. 4.7). 
Auf der Ackerfläche war die durchschnittliche Vitalität der in der zweiten Vegetationsperiode lebenden Aspen und des Pappelstecklinge "Max 4" mit Hydrogel signifikant schlechter als bei den Kontrollpflanzen $(p \leq 0,05)$

Auf der Grünlandfläche hatten die mit Hydrogel gepflanzten Aspen eine signifikant bessere durchschnittliche Vitalität aufzuweisen als die Kontrollpflanzen $(p \leq 0,05)$. Auch die Aspen, die mit der kombinierten Anwendung von Hydrogel und Mykorrhiza gepflanzt wurde, wiesen eine signifikant bessere Vitalität auf $(p \leq 0,05)$. In der ersten Vegetationsperiode wiesen die Aspen mit diesen Kombinationen an Bodenhilfsstoffen bereits eine bessere Vitalität als die Kontrollpflanzen auf, war aber noch nicht signifikant.

Die Vitalität der Robinie war auf der Grünlandfläche signifikant schlechter, wenn die Gehölze vor der Pflanzung mykorrhiziert wurden $(p \leq 0,05)$. Die im ersten Jahr noch signifikant bessere Vitalität mit Hydrogel bzw. Hydrogel und Mykorrhiza war im zweiten Jahr nicht mehr feststellbar bzw. nicht mehr signifikant.

Auch die mykorrhizierten Weidenstecklinge wiesen in der zweiten Vegetationsperiode eine signifikant schlechtere durchschnittliche Vitalität auf als die Kontrollpflanzen $(p \leq 0,05)$.

\subsubsection{Interspezifische Konkurrenz}

Die Anwuchsraten der Pappelstecklinge "Max 4" und "NE 42" korrelierten negativ mit der Trockenbiomasse der einkeimblättrigen Kräuter auf der Fläche. Bei "Max 4" war diese Korrelation nicht signifikant, im Gegensatz zur Korrelation zwischen der Trockenbiomasse des Deutschen Weidelgrases (Lolium perenne) und der Anwuchsrate des Stecklings; der Korrelationskoeffizient nach Pearson betrug -0,79. Die Anwuchsrate von "NE 42" korrelierte signifikant negativ mit der Gesamtbiomasse der einkeimblättrigen Kräuter. Der Korrelationskoeffizient nach Pearson betrug -0,81. Die Anwuchsrate des Pappelhybrids "NE 42" korrelierte ebenfalls mit der Trockenmasse des Deutschen Weidelgrases, allerdings war diese Korrelation nicht signifikant. 
Die Regressionsanalyse bestätigte einen linearen Zusammenhang zwischen den Anwuchsprozenten der Pappelhybriden mit der grasartigen Bodenvegetation (Abb. 4.9). Je höher das Aufkommen der einkeimblättrigen Kräuter war, desto weniger Pappelstecklinge sind nach der Pflanzung ausgetrieben. Bei dem Pappelhybrid "Max 4" bezog sich dieser lineare Zusammenhang besonders auf das Auftreten des Deutschen Weidelgrases. Je mehr Biomasse vom Deutschen Weidelgras gebildet wurde, desto geringer war die Anzahl an austreibenden Stecklingen.

Zwischen den Anwuchsraten der anderen Baumarten und der Konkurrenzvegetation gab es keine signifikante Korrelation, aber zwischen dem Anteil an vitalen Gehölzen der Vitalitätsstufe 1 und der aufgekommenen natürlichen Begleitvegetation.

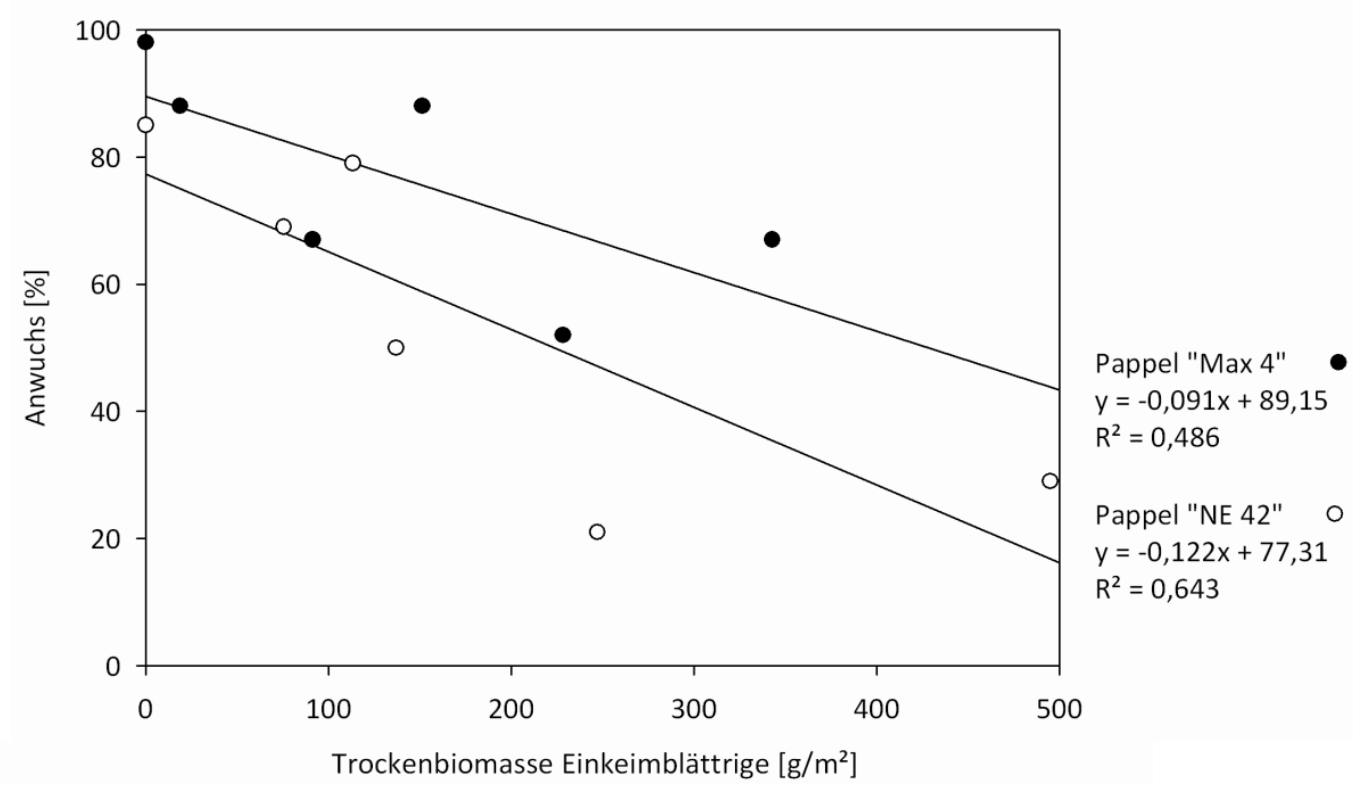

\section{Abb. 4.9: Anwuchsprozente der Pappelstecklinge in der ersten Vegetationsperiode in Abhängigkeit von der Trockenbiomasse der einkeimblättrigen Kräuter}

Der Anteil an vitalen Aspen und Robinien korrelierte negativ mit der Biomasse einkeimblättriger Kräuter. Bei Aspe war die Korrelation mit dem Aufkommen einkeimblättriger Kräuter signifikant; der Korrelationskoeffizienten nach Pearson lag bei -0,85. Bei fehlender Grasdecke (gemähte Ackerfläche) betrug der Anteil vitaler Aspen 60 \%, welcher mit zunehmender Grasvegetation auf bis zu 20 \% absank. Der Anteil an vitalen Robinien wurde signifikant negativ von der Trockenbiomasse des 
Deutschen Weidelgrases beeinflusst. Die Korrelation zwischen vitalen Robinien und der Gesamtbiomasse der einkeimblättrigen Kräuter war dagegen nicht signifikant. Der Korrelationskoeffizient nach Pearson zwischen der Trockenmasse des Deutschen Weidelgrases und dem Anteil vitaler Robinien betrug -0,76. Die Regressionsgraden zwischen dem Anteil vitaler Aspen und Robinien und der Biomasse einkeimblättriger Kräuter ist in Abb. 4.10 dargestellt. Je höher das Aufkommen einkeimblättriger Kräuter auf der Fläche war, desto geringer war der relative Anteil von Aspen und Robinien in der Vitalitätsstufe 1 ohne erkennbare Schäden.

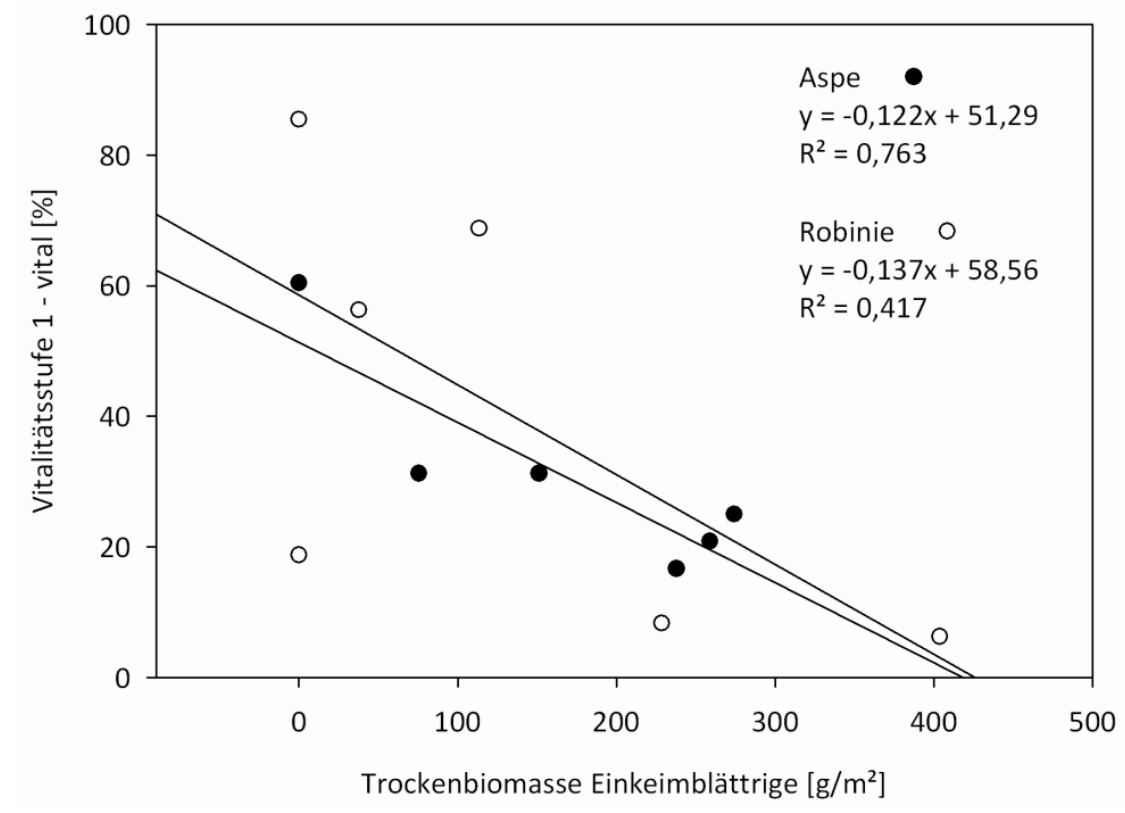

Abb. 4.10: Prozentuale Anteile an vitalen Aspen und Robinien in der ersten Vegetationsperiode in Abhängigkeit von der Trockenbiomasse der einkeimblättrigen Kräuter

In der zweiten Vegetationsperiode war eine signifikante Korrelation war zwischen der Überlebensrate der Aspe mit dem Deckungsgrad der Begleitvegetation zu finden; der Korrelationskoeffizient nach Pearson betrug -0,73. Der Korrelationskoeffizient zwischen der Überlebensrate der Robinie und dem Deckungsgrad der Begleitvegetation betrug -0,69, war allerdings nicht signifikant. Der lineare Zusammenhang zwischen Deckungsgrad und Anwuchs ist in Abb. 4.11 dargestellt. Je 
höher der Deckungsgrad der Konkurrenzvegetation war, desto geringer war die Überlebensrate an Aspen und Robinien.

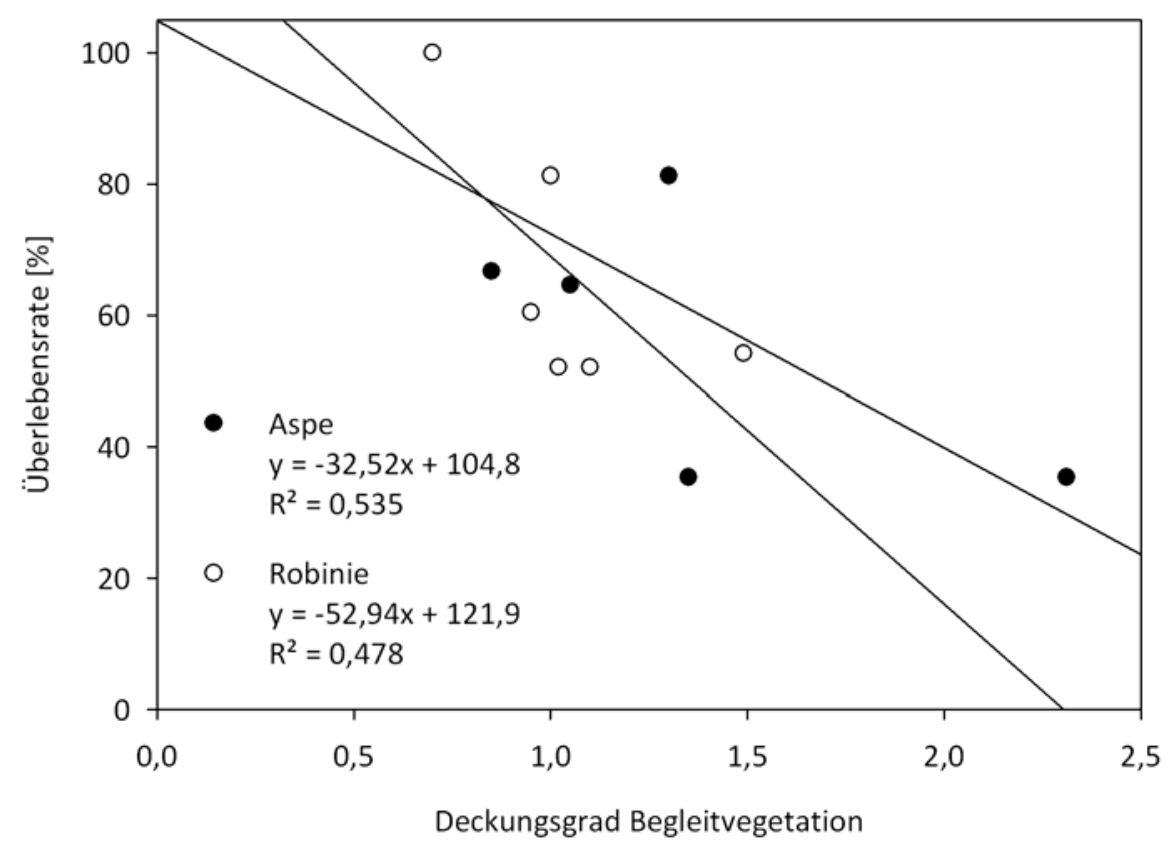

Abb. 4.11: Überlebensrate von Aspe und Robinie nach der zweiten Vegetationsperiode in Abhängigkeit vom Deckungsgrad der Begleitvegetation auf der Versuchsfläche Sudheide in Gütersloh

\subsubsection{Mäuseschäden}

Insgesamt fielen $12 \%$ der Pflanzen durch Mäuseschäden aus, das sind etwa 30 \% des Gesamtausfalls an Pflanzen. Die Ausfallrate durch Mäuse betrug auf der Ackerfläche $9 \%$ und auf der Grünlandfläche $16 \%$.

Insbesondere die Pappeln, Robinie und Schwarzerle wurden von den Mäusen benagt. Auf der Ackerfläche betrug die Ausfallrate der Aspen durch Mäuseschäden 11 \% und auf der Grünlandfläche 13 \%. Beim Pappelsteckling "Max 4" fielen 7 \% der Stecklinge auf Ackerfläche und 31 \% auf der Grünlandfläche aus. 19 \% der Pappelstecklinge „NE 42" fielen den Mäusen auf der Ackerfläche und 14 \% auf der Grünlandfläche zum Opfer. Bei der Robinie betrug die Ausfallrate durch Mäusefraß $27 \%$ auf der Ackerfläche und $17 \%$ auf der Grünlandfläche. Die Schwarzerle hatte auf der Ackerfläche eine Ausfallrate durch Mäuse von $13 \%$. 


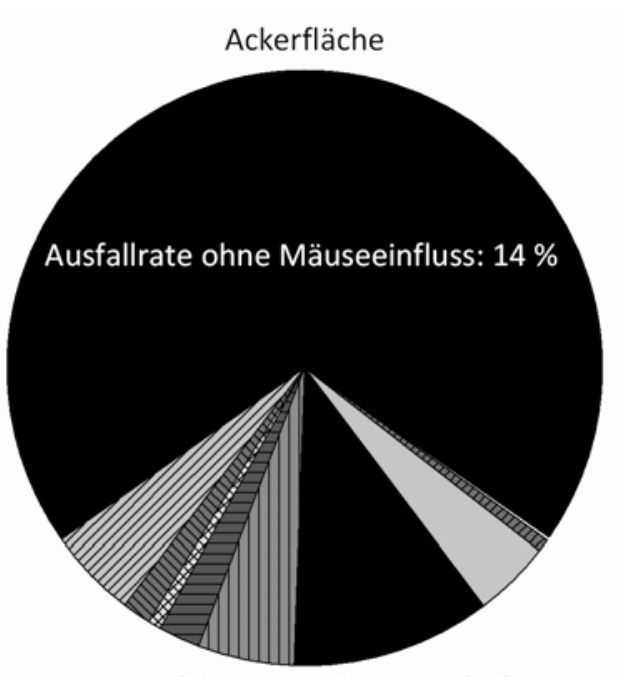

Ausfallrate durch Mäusefraß: $9 \%$

एIIII Aspe: $11 \%$

МIIV Bergahorn: $4 \%$

Eberesche $2 \%$

Pappel "Max 4": 7 \%

피니 Pappel "NE 42": $19 \%$

Robinie: $27 \%$

$\square$ Schwarzerle: $13 \%$

WIIn Weide "Turbo": $2 \%$

बIVW Winterlinde: $0 \%$

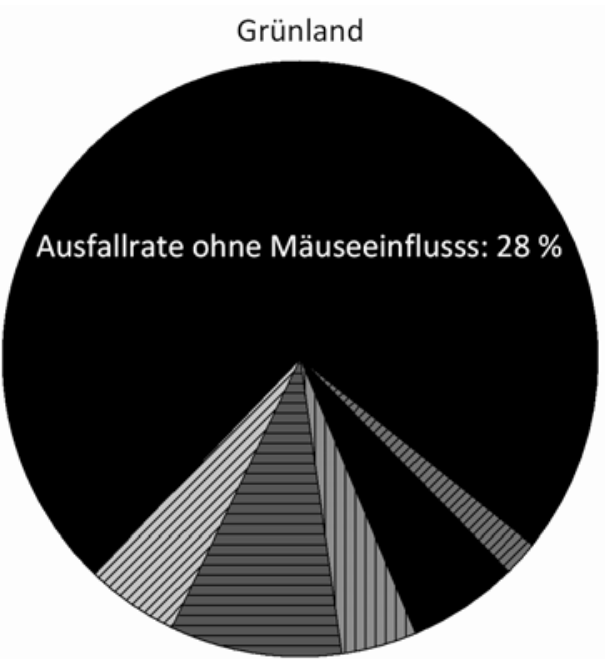

Ausfallrate durch Mäusefraß: $16 \%$

चIII Aspe: $13 \%$

Pappel "Max 4": $31 \%$

피미 Pappel "NE 42": $14 \%$

Robinie: $17 \%$

चIII Weide "Turbo": $5 \%$

Abb. 4.12: Durch Mäusefraß verursachte Pflanzenausfälle auf der Acker- und Grünlandfläche Sudheide in Gütersloh nach zwei Vegetationsperioden

\subsubsection{Zuwachsmessungen}

\subsubsection{Flächenvornutzung}

Auf der Fläche Sudheide zeigte sich ein signifikanter Einfluss der Flächenvornutzung auf den Höhenzuwachs der Gehölze in der ersten Vegetationsperiode (Abb. 4.13).

Die Wuchsunterschiede der Gehölze in Abhängigkeit von der Vornutzung waren über alle Baumarten hinweg hoch- bzw. höchstsignifikant. So erreichten die Gehölze auf der Ackerfläche etwa 2-3-mal höhere Zuwächse als die gleichen auf dem Grünland gepflanzten Gehölzarten. Die Aspe wuchs auf der Ackerfläche $35 \mathrm{~cm}$ in der ersten Vegetationsperiode, der Pappelsteckling "Max 4" wies auf der Ackerfläche einen Zuwachs von 1,10 m auf, "NE 42" wuchs im ersten Jahr um etwa $80 \mathrm{~cm}$ und Robinie um $100 \mathrm{~cm}$. Die beste Höhenwuchsleistung zeigte die Weide, die auf der Ackerfläche eine Höhe von etwa 1,55 m nach der ersten Vegetationsperiode erreichte. Die 
Höhenzuwächse auf der Grünlandfläche waren signifikant niedriger mit $15 \mathrm{~cm}$ Zuwachs der Aspe, $55 \mathrm{~cm}$ bei Pappel "Max 4", $45 \mathrm{~cm}$ bei Pappel "NE 42 ", $30 \mathrm{~cm}$ bei Robinie und $95 \mathrm{~cm}$ bei Weide.

Die für Kurzumtriebsplantagen eher ungewöhnlichen Baumarten, welche nur auf der Ackerfläche angepflanzt wurden, wiesen in der ersten Vegetationsperiode einen geringeren Zuwachs auf als die schnellwachsenden Pappeln, Robinien und Weiden (Abb. 4.13). Bergahorn hatte in der ersten Vegetationsperiode einen Zuwachs von nur $10 \mathrm{~cm}$ und die Eberesche von etwa $30 \mathrm{~cm}$. Die Schwarzerle wuchs im ersten Standjahr $25 \mathrm{~cm}$ und die Winterlinde um die $15 \mathrm{~cm}$.

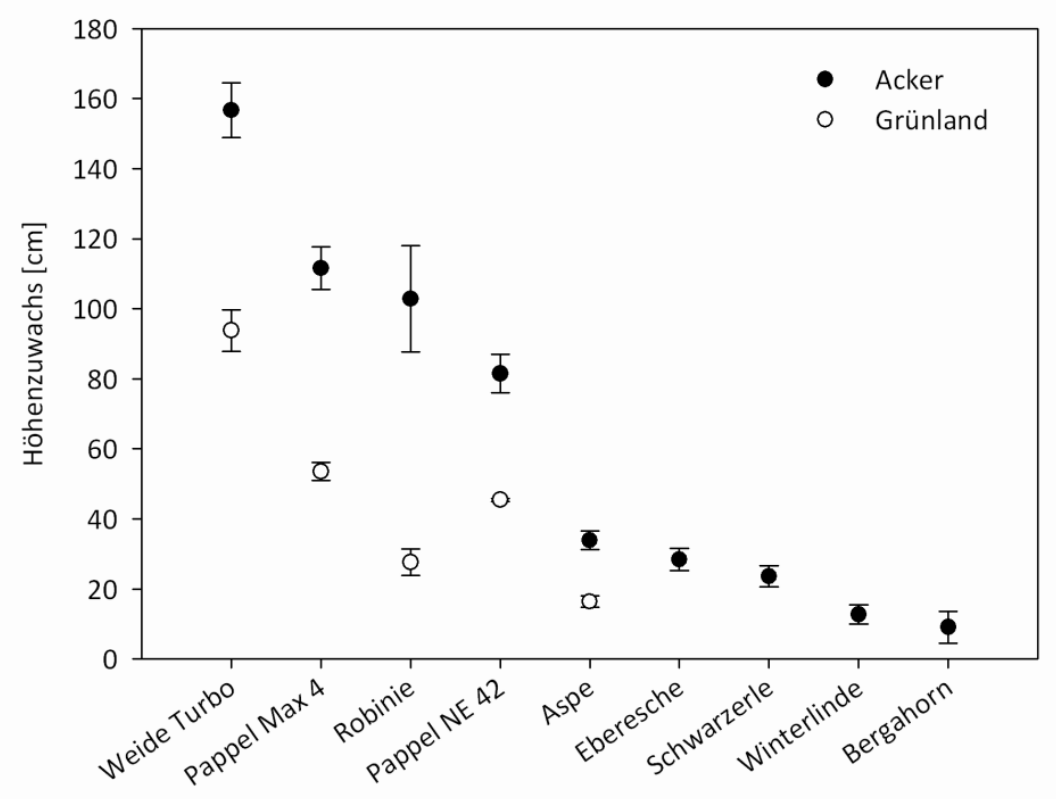

Abb. 4.13: Höhenzuwachs der Kontrollpflanzen in der ersten Vegetationsperiode 2007 auf der Versuchsfläche Sudheide bei Gütersloh ( \pm Standardabweichung)

Auch nach der zweiten Vegetationsperiode zeigt sich ein signifikant besserer Höhenzuwachs der meisten Gehölze auf der Ackerfläche als auf dem Grünland (Abb. 4. 14).

Die Aspe wuchs im zweiten Standjahr um $75 \mathrm{~cm}$ auf eine Endhöhe von $110 \mathrm{~cm}$. Auf dem Grünland hatte die Aspe einen geringeren Höhenzuwachs von $85 \mathrm{~cm}$. Bergahorn wuchs im zweiten Jahr mit $35 \mathrm{~cm}$ dreimal so viel wie im ersten Jahr und erreichte einen Gesamtzuwachs von $45 \mathrm{~cm}$. Der Zuwachs der Eberesche war im zweiten Jahr doppelt 
so hoch wie im ersten Jahr. Die Eberesche wuchs um die $50 \mathrm{~cm}$ und erreichte damit einen Gesamtzuwachs von $75 \mathrm{~cm}$.

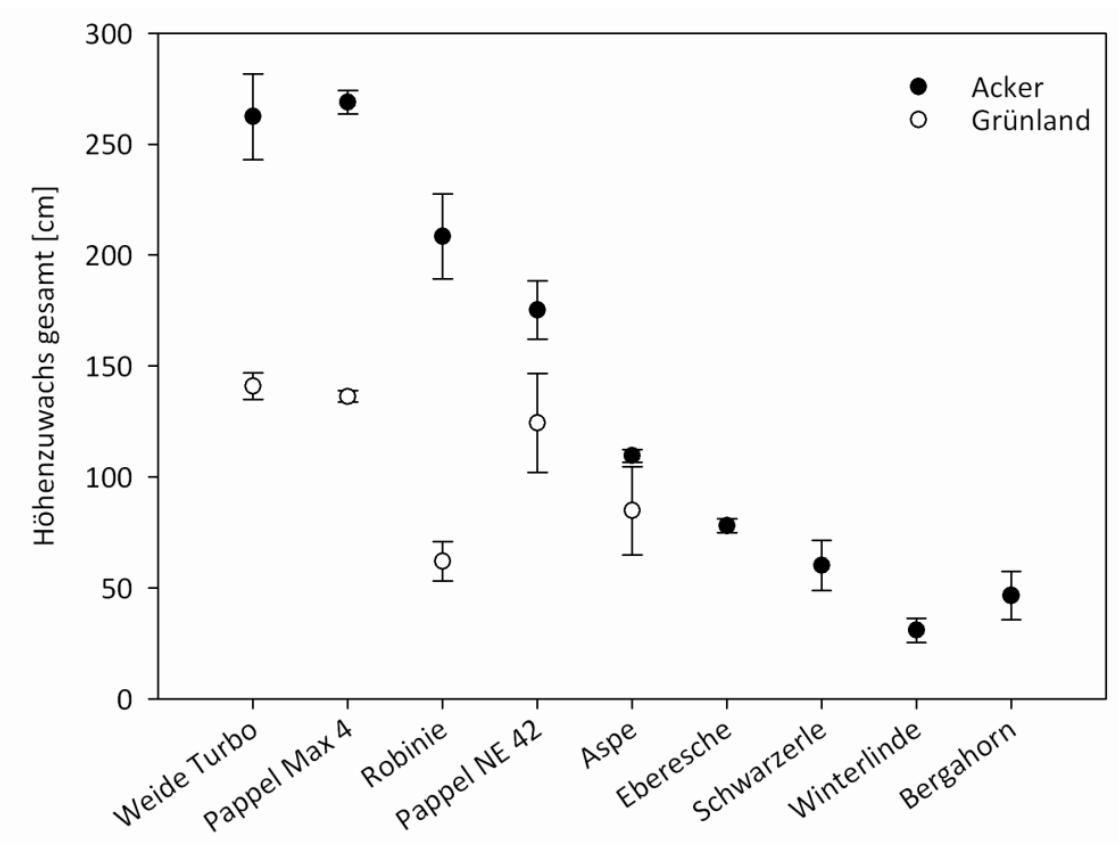

Abb. 4.14: Höhenzuwachs der Gehölze auf der Versuchsfläche Sudheide in den ersten beiden Vegetationsperioden

Die Pappel "Max 4" wies in der zweiten Vegetationsperiode mit $160 \mathrm{~cm}$ den größten Höhenzuwachs auf und erreichte mit insgesamt $270 \mathrm{~cm}$ den höchsten Gesamtzuwachs der gepflanzten Gehölze. Die Pappel "NE 42" wuchs im zweiten Standjahr mit $95 \mathrm{~cm}$ nur knapp mehr als im ersten Jahr und erreichte eine Endhöhe von $175 \mathrm{~cm}$. Auf der Grünlandfläche erreichten beide Pappelhybriden eine Höhe von etwa $130 \mathrm{~cm}$ nach der zweiten Vegetationsperiode. Der Zuwachs der Robinie blieb mit $105 \mathrm{~cm}$ konstant gegenüber der ersten Vegetationsperiode und erreichte somit einen Gesamtzuwachs von $210 \mathrm{~cm}$ nach zwei Standjahren. Auf der Grünfläche war der Gesamtzuwachs der Robinie mit nur $60 \mathrm{~cm}$ signifikant geringer als auf der Ackerfläche. Die Schwarzerle legte im zweiten Jahr um die $35 \mathrm{~cm}$ an Höhe zu und wuchs in den zwei Jahren insgesamt $60 \mathrm{~cm}$. Die Weide hatte als einzige Baumart einen geringeren Zuwachs in der zweiten als in der ersten Vegetationsperiode. Die Weide wuchs auf der ehemaligen Ackerfläche $105 \mathrm{~cm}$ in der zweiten Vegetationsperiode und erreichte eine 
Endhöhe von $260 \mathrm{~cm}$. Auf der Grünlandfläche war die Höhe der Weide mit $160 \mathrm{~cm}$ nach zwei Wachstumsperioden signifikant niedriger als auf der Ackerfläche. Die Winterlinde wies nach zwei Jahren mit $30 \mathrm{~cm}$ den geringsten Gesamtzuwachs auf; im zweiten Jahr wuchs diese um $20 \mathrm{~cm}$, doppelt so viel wie im ersten Standjahr nach der Pflanzung.

\subsubsection{Begleitwuchsregulierung}

Das Mähen der Fläche führte in der ersten Vegetationsperiode tendenziell zu einem verbesserten Wuchs der Pflanzen, der Unterschied zum Höhenzuwachs der Kontrollpflanzen war aber zumeist nicht signifikant (Tab. 4.8). Bei der Aspe hatte das Mähen auf der Grünfläche zwischen den Pflanzreihen einen signifikant positiven Einfluss auf den Höhenzuwachs in der ersten Vegetationsperiode. Und der Höhenzuwachs der Weiden wurde sowohl auf der Acker- als auch auf der Grünlandfläche signifikant positiv durch das Mähen der Fläche beeinflusst.

Tab. 4.8: Höhenzuwachs $(\mathrm{cm})$ der Gehölze auf der Acker- und Grünlandfläche in der ersten Vegetationsperiode in Abhängigkeit von der Begleitwuchsregulierung ( \pm Standardfehler; ${ }^{*}$ signifikante Mittelwert-Differenz zur Kontrolle mit $p \leq 0,05$ )

\begin{tabular}{|c|c|c|c|c|c|c|c|}
\hline & & \multicolumn{2}{|c|}{ Kontrolle } & \multicolumn{2}{|c|}{ Mähen } & \multicolumn{2}{|c|}{ Nutzpflanzen } \\
\hline & & $\mathrm{cm}$ & \pm & $\mathrm{cm}$ & \pm & $\mathrm{cm}$ & \pm \\
\hline \multirow{9}{*}{ 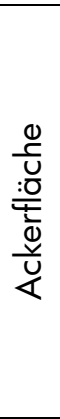 } & Aspe & 34 & 2,7 & 35 & 1,3 & $26^{*}$ & 2,9 \\
\hline & Bergahorn & 9 & 4,6 & 11 & 1,4 & 11 & 2,7 \\
\hline & Eberesche & 28 & 3,2 & 23 & 2,8 & 22 & 2,2 \\
\hline & Pappel „Max 4“ & 112 & 6,0 & 112 & 2,2 & 105 & 15,6 \\
\hline & Pappel "NE 42" & 81 & 5,5 & 91 & 6,8 & 84 & 8,8 \\
\hline & Robinie & 103 & 15,2 & 118 & 2,9 & $87^{*}$ & 2,0 \\
\hline & Schwarzerle & 24 & 3,1 & 13 & 5,2 & 23 & 4,4 \\
\hline & Weide "Turbo" & 157 & 7,8 & $182 *$ & 1,9 & 156 & 10,7 \\
\hline & Winterlinde & 13 & 2,8 & 9 & 1,6 & 11 & 1,5 \\
\hline \multirow{5}{*}{ 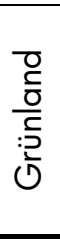 } & Aspe & 16 & 1,7 & $24 *$ & 2,0 & 14 & 0,8 \\
\hline & Pappel "Max 4" & 54 & 2,6 & 49 & 5,2 & 44 & 2,3 \\
\hline & Pappel "NE 42" & 45 & 0,4 & 47 & 2,9 & 25 & 12,5 \\
\hline & Robinie & 28 & 3,8 & 39 & 3,2 & 28 & 5,2 \\
\hline & Weide "Turbo" & 94 & 5,9 & $107 *$ & 7,5 & $65^{*}$ & 4,5 \\
\hline
\end{tabular}


Die eingesäten Nutzpflanzen zeigten dagegen einen negativen Einfluss auf den Höhenzuwachs der Gehölze in der ersten Vegetationsperiode, aber auch hier war der Unterschied zu den Kontrollpflanzen meistens nicht signifikant. Auf der Ackerfläche hatten die Aspen und Robinien mit eingesäten Nutzpflanzen einen signifikant geringeren Höhenzuwachs nach der ersten Vegetationsperiode aufzuweisen als die Kontrollpflanzen. Auf der Grünlandfläche war der Effekt der eingesäten Nutzpflanzen nur bei der Weide signifikant negativ.

Tab. 4.9: Gesamthöhenzuwächse der Gehölze auf der Versuchsfläche Sudheide nach zwei Vegetationsperioden in Abhängigkeit von der Begleitwuchsregulierung ( \pm Standardfehler; ${ }^{*}$ signifikante Mittelwert-Differenz zur Kontrolle mit $p \leq 0,05$ )

\begin{tabular}{|c|c|c|c|c|c|c|c|}
\hline & \multicolumn{2}{|c|}{ Kontrolle } & \multicolumn{2}{|c|}{ Mähen } & \multicolumn{2}{|c|}{ Nutzpflanzen } \\
\hline & & $\mathrm{cm}$ & \pm & $\mathrm{cm}$ & \pm & $\mathrm{cm}$ & \pm \\
\hline \multirow{9}{*}{ 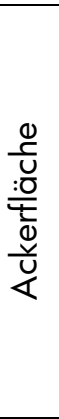 } & Aspe & 110 & 2,8 & 103 & 5,2 & $68^{*}$ & 7,9 \\
\hline & Bergahorn & 47 & 10,9 & $82 *$ & 7,3 & 64 & 9,4 \\
\hline & Eberesche & 78 & 3,2 & 71 & 1,2 & $64^{*}$ & 5,4 \\
\hline & Pappel "Max 4" & 269 & 5,2 & 247 & 17,1 & $214^{*}$ & 12,5 \\
\hline & Pappel "NE 42" & 175 & 13,2 & 200 & 10,4 & $142 *$ & 13,4 \\
\hline & Robinie & 208 & 19,0 & 164 & 10,5 & $138 *$ & 9,2 \\
\hline & Schwarzerle & 60 & 11,3 & $37 *$ & 12,6 & 105 & 26,5 \\
\hline & Weide "Turbo" & 262 & 19,3 & 244 & 9,0 & 230 & 10,2 \\
\hline & Winterlinde & 31 & 5,5 & 22 & 1,5 & 23 & 1,8 \\
\hline \multirow{5}{*}{ 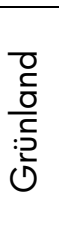 } & Aspe & 85 & 19,9 & 55 & 10,8 & 57 & 7,3 \\
\hline & Pappel "Max 4" & 136 & 2,6 & 134 & 15,7 & 107 & 4,1 \\
\hline & Pappel "NE 42" & 124 & 22,3 & 135 & 6,9 & 125 & 17,0 \\
\hline & Robinie & 62 & 8,8 & $99 *$ & 3,8 & 57 & 0,3 \\
\hline & Weide "Turbo" & 141 & 5,9 & 144 & 3,7 & $116^{*}$ & 9,4 \\
\hline
\end{tabular}

Der positive Einfluss des Mähens, der nach der ersten Vegetationsperiode noch deutlich sichtbar war, hatte sich nach dem zweiten Standjahr weitestgehend relativiert (Tab. 4.9). Mit Ausnahmen zeigte sich durch das Mähen keine positive Tendenz der Zuwächse mehr. Ausnahmen waren der Bergahorn, welcher auf der Ackerfläche signifikant höhere Gesamtzuwächse hatte, wenn zwischen den Pflanzreihen gemäht wurde. Der Gesamtzuwachs der Schwarzerle wurde durch das Mähen sogar vermindert. Auf der gemähten Grünlandfläche hatte die Robinie einen um 30 \% höheren Gesamtzuwachs im Vergleich zur Kontrollfläche.

Es zeigte sich nach der zweiten Vegetationsperiode ein signifikant negativer Einfluss der eingesäten Nutzpflanzen auf den Höhenzuwachs der Aspe, Eberesche, Pappel 
"Max 4" und "NE 42" auf der Ackerfläche und der Weide "Turbo" auf dem Grünland.

\subsubsection{Bodenhilfsstoffe}

In Tab. 4.10 sind die mittleren Höhenzuwächse der gepflanzten Gehölze in Abhängigkeit von den beigemischten Bodenhilfsstoffen aufgelistet. Die Beigabe von Hydrogel hatte mit zwei Ausnahmen keinen signifikanten Einfluss auf den Höhenzuwachs der Gehölze in der ersten Vegetationsperiode. Eine Ausnahme war die Aspe auf der Grünlandfläche, deren Höhenzuwachs mit Hydrogel nur die Hälfte des Höhenzuwachses der Kontrollpflanzen betrug. Einen positiven signifikanten Einfluss hatte die Hydrogel-Beimischung auf den Höhenzuwachs der Robinie, welche auf der Grünlandfläche gepflanzt wurde. Mit Hydrogel wuchs die Robinie in der ersten Vegetationsperiode mehr als 50 \% höher als die Kontrollpflanzen.

Tab. 4.10: mittlere Höhenzuwächse der Gehölze auf der Versuchsfläche Sudheide nach der ersten Vegetationsperiode in Abhängigkeit von den applizierten Bodenhilfsstoffen ( \pm Standardfehler; ${ }^{*}$ signifikante Mittelwert-Differenz zur Kontrolle mit $p \leq 0,05$ )

\begin{tabular}{|c|c|c|c|c|c|c|c|c|c|}
\hline & \multicolumn{2}{|c|}{ Kontrolle } & \multicolumn{2}{|c|}{ Hydrogel } & \multicolumn{2}{|c|}{ Mykorrhiza } & \multicolumn{2}{|c|}{$\begin{array}{c}\text { Hydro. + } \\
\text { Myk. }\end{array}$} \\
\hline & & $\mathrm{cm}$ & \pm & $\mathrm{cm}$ & \pm & $\mathrm{cm}$ & \pm & $\mathrm{cm}$ & \pm \\
\hline \multirow{9}{*}{ 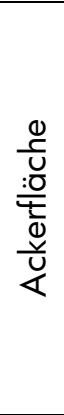 } & Aspe & 35 & 2,9 & 32 & 4,6 & 34 & 6,0 & $21^{*}$ & 4,0 \\
\hline & Bergahorn & 9 & 3,7 & 12 & 4,6 & 10 & 0,9 & 12 & 2,2 \\
\hline & Eberesche & 29 & 3,0 & 22 & 3,6 & 26 & 5,5 & 34 & 4,9 \\
\hline & Pappel „Max 4" & 111 & 4,5 & 124 & 7,8 & $126 *$ & 4,9 & 108 & 3,3 \\
\hline & Pappel "NE 42" & 81 & 3,7 & 84 & 5,7 & $102 *$ & 5,5 & 91 & 7,9 \\
\hline & Robinie & 108 & 6,9 & 100 & 10,2 & 118 & 8,3 & 109 & 10,1 \\
\hline & Schwarzerle & 24 & 3,0 & 31 & 8,1 & 22 & 6,0 & 17 & 3,3 \\
\hline & Weide "Turbo" & 157 & 4,4 & 151 & 7,8 & 144 & 9,5 & 161 & 7,2 \\
\hline & Winterlinde & 14 & 1,6 & 16 & 2,7 & 14 & 2,6 & $5 *$ & 1,7 \\
\hline \multirow{5}{*}{ 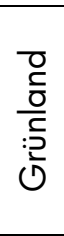 } & Aspe & 17 & 1,8 & $8^{*}$ & 3,0 & 9 & 2,1 & 24 & 4,2 \\
\hline & Pappel "Max 4" & 54 & 3,8 & 45 & 6,9 & 51 & 9,1 & 41 & 8,4 \\
\hline & Pappel "NE 42" & 45 & 4,4 & --- & -- & 54 & 1,7 & 55 & 7,4 \\
\hline & Robinie & 28 & 3,3 & $45^{*}$ & 6,3 & 18 & 3,7 & 39 & 2,8 \\
\hline & Weide "Turbo" & 94 & 3,7 & 91 & 6,9 & 85 & 10,3 & 88 & 5,6 \\
\hline
\end{tabular}


Die Mykorrhizierung konnte die Höhenzuwächse der Pappelstecklinge in der ersten Vegetationsperiode verbessern. Die Pappelstecklinge "Max 4" und "NE 42" hatten in der ersten Vegetationsperiode einen um 10-35\% höheren Zuwachs infolge der Mykorrhizierung.

Die Kombination von Hydrogel und Mykorrhiza hatte auf der Ackerfläche einen signifikant negativen Effekt auf den Höhenzuwachs der Aspe und der Winterlinde.

Nach der zweiten Vegetationsperiode war der Höhenzuwachs der Aspe (Populus tremula) mit Hydrogelapplikation auf der Ackerfläche um ein Drittel geringer im Vergleich zu den Kontrollpflanzen (Tab. 4.11). Auch die Weide wies auf der Ackerfläche einen signifikant geringeren Gesamthöhenzuwachs mit Hydrogel auf. Auf der Grünlandfläche war der Höhenzuwachs des Pappelstecklings "Max 4" nach zwei Jahren nur halb so groß, wenn bei der Pflanzung Hydrogel appliziert wurde. Lediglich die Robinie auf dem Grünland wies einen signifikant positiven Effekt des Hydrogels auf den Gesamthöhenzuwachs auf. Die mit Hydrogel gepflanzten Robinien waren nach zwei Jahren doppelt so viel gewachsen wie die Kontrollpflanzen.

Die Mykorrhizierung der Gehölze zeigte nach zwei Vegetationsperioden einen signifikant positiven Effekt auf den Gesamthöhenzuwachs von Bergahorn, Eberesche und dem Pappelsteckling "Max 4" auf der Ackerfläche und einen signifikant negativen Effekt auf den Gesamthöhenzuwachs der Weide "Turbo" auf der Grünlandfläche. Der Zuwachs des Bergahorns war infolge der Mykorrhizierung doppelt so hoch wie der Zuwachs der Kontrollpflanzen. Der Gesamthöhenzuwachs der mykorrhizierten Eberesche war nach zwei Jahren 50 \% höher als bei den Kontrollpflanzen. Der Zuwachs der Pappel "Max 4" wurde durch das applizierte Mykorrhizapulver um 25 \% gesteigert. Im Gegensatz zu der Ackerfläche hatte die Mykorrhizierung auf der Grünfläche einen negativen Einfluss auf den Gesamthöhenzuwachs der Gehölze nach zwei Wachstumsperioden, welcher aber nur bei der Weide signifikant war. Der Gesamthöhenzuwachs der mykorrhizierten Weidenstecklinge betrug nur $70 \%$ des Zuwachses der Kontrollpflanzen. 
Die Kombination aus Hydrogel und Mykorrhiza zeigte nach zwei Vegetationsperioden keinen signifikanten Effekt auf den Gesamthöhenzuwachs der gepflanzten Gehölze.

Tab. 4.11: Gesamthöhenzuwächse der Gehölze auf der Versuchsfläche Sudheide nach zwei Vegetationsperioden in Abhängigkeit von den applizierten Bodenhilfsstoffen ( \pm Standardfehler; * signifikante Mittelwert-Differenz zur Kontrolle mit $p \leq 0,05$ )

\begin{tabular}{|c|c|c|c|c|c|c|c|c|c|}
\hline & \multicolumn{2}{|c|}{ Kontrolle } & \multicolumn{2}{|c|}{ Hydrogel } & \multicolumn{2}{|c|}{ Mykorrhiza } & \multicolumn{2}{|c|}{$\begin{array}{c}\text { Hydro. + } \\
\text { Myk. }\end{array}$} \\
\hline & & $\mathrm{cm}$ & \pm & $\mathrm{cm}$ & \pm & $\mathrm{cm}$ & \pm & $\mathrm{cm}$ & \pm \\
\hline \multirow{9}{*}{ 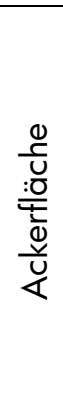 } & Aspe & 92 & 6,6 & $62 *$ & 11,5 & 87 & 14,6 & 113 & 10,1 \\
\hline & Bergahorn & 35 & 7,6 & 44 & 13,8 & $77^{*}$ & 15,8 & 24 & 9,3 \\
\hline & Eberesche & 75 & 4,7 & 65 & 3,9 & $119 *$ & 11,5 & 89 & 10,0 \\
\hline & Pappel „Max 4" & 231 & 16,4 & 165 & 31,4 & $292 *$ & 20,9 & 268 & 16,2 \\
\hline & Pappel "NE 42" & 132 & 10,6 & 96 & 18,6 & 142 & 26,5 & 128 & 23,9 \\
\hline & Robinie & 146 & 11,3 & 164 & 14,9 & 163 & 14,5 & 162 & 22,3 \\
\hline & Schwarzerle & 24 & 5,3 & 42 & 8,2 & 49 & 20,1 & 21 & 5,7 \\
\hline & Weide "Turbo" & 260 & 8,8 & $224^{*}$ & 14,1 & 234 & 17,0 & 270 & 12,4 \\
\hline & Winterlinde & 26 & 3,4 & 31 & 4,2 & 31 & 3,0 & 15 & 2,9 \\
\hline \multirow{5}{*}{$\frac{\text { Oे }}{\frac{0}{0}}$} & Aspe & 62 & 8,2 & 85 & 12,1 & 48 & 14,9 & 73 & 9,8 \\
\hline & Pappel "Max 4" & 85 & 10,2 & $41^{*}$ & 14,9 & 57 & 15,7 & 50 & 15,9 \\
\hline & Pappel "NE 42" & 29 & 8,9 & - & - & 14 & 8,6 & 57 & 17,6 \\
\hline & Robinie & 45 & 5,5 & $85^{*}$ & 12,1 & 29 & 7,4 & 50 & 11,1 \\
\hline & Weide "Turbo" & 129 & 7,0 & 115 & 18,6 & $94^{*}$ & 16,2 & 132 & 18,1 \\
\hline
\end{tabular}

\subsubsection{Interspezifische Konkurrenz}

Die deutlichen Wuchsunterschiede auf Acker- und Grünlandfläche in der ersten Vegetationsperiode sind mit der Intensität der natürlichen Begleitvegetation auf den unterschiedlich vorgenutzten Flächen zu erklären. Die Zuwächse der Pflanzenhöhe und des Wurzelhalsdurchmessers (WHD) der Gehölze, die auf beiden Flächen gepflanz† wurden, korrelierten signifikant mit der Trockenbiomasse der Begleitvegetation, insbesondere der einkeimblättrigen Kräuter. Bei den Zuwächsen der Aspe zeigte sich eine Korrelation mit der Trockenbiomasse der einkeimblättrigen Kräuter von -0,71 und eine deutliche Korrelation mit dem Aufkommen des Deutschen Weidelgrases (Lolium perenne) von -0,76. Die Höhenzuwachse der Pappelsteckling "Max 4" und "NE 42" korrelierten mit einem Korrelationskoeffizient von $-0,77$ ebenfalls signifikant negativ mit dem Aufkommen einkeimblättriger Kräuter. Je höher die gebildete Trockenmasse 
einkeimblättriger Kräuter war, desto geringer war der Höhenzuwachs der Pappelstecklinge "Max 4" und "NE 42" (Abb. 4.15).

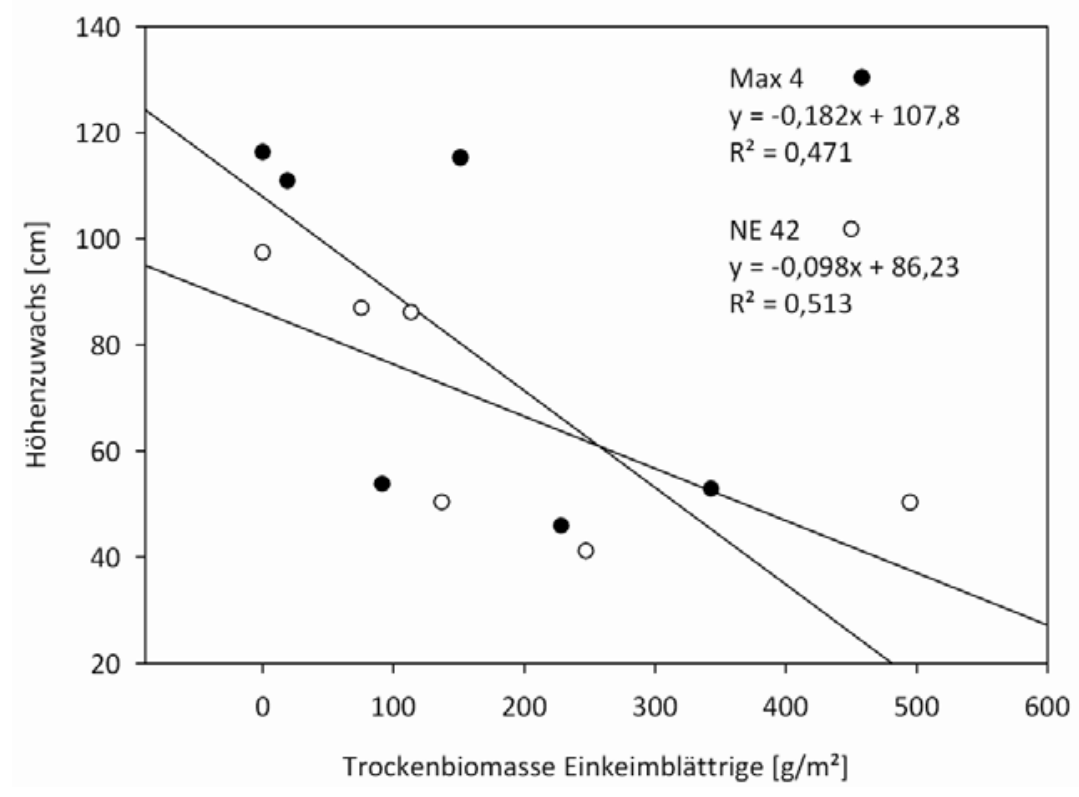

Abb. 4.15: Höhenzuwachs der Pappelstecklinge in der ersten Vegetationsperiode in Abhängigkeit von der Trockenbiomasse der einkeimblättrigen Kräuter

Der WHD der Pappel "NE 42" korrelierte mit einem Korrelationskoeffizienten von $-0,88$ ebenfalls signifikant negativ mit der Trockenmasse der einkeimblättrigen Kräuter. Der WHD der Robinie korrelierte mit einem Korrelationskoeffizienten von -0,85 mit der Trockenmasse des Deutschen Weidelgrases. Zwischen den Höhenzuwächsen der Robinie und der Begleitvegetation gab es allerdings keine signifikante Korrelation. Bei Weide zeigte sich eine signifikante Korrelation der Höhe und des WHDs mit der Trockenbiomasse einkeimblättriger Kräuter mit einem Korrelationskoeffizienten von -0,75. Der Korrelationskoeffizient mit dem Deutschen Weidelgras lag für Weide bei $-0,78$ und war signifikant negativ.

Der Zuwachs in der zweiten Vegetationsperiode war unabhängig von der Konkurrenzvegetation desselben Jahres. Die Konkurrenzvegetation im ersten Jahr beeinflusst aber noch den Höhenzuwachs im zweiten Standjahr, die Korrelation war jedoch nicht signifikant. Die Korrelation zwischen der Begleitvegetation in der ersten Vegetationsperiode mit dem Gesamthöhenzuwachs war teilweise signifikant. Der 
Höhenzuwachs der Aspe korrelierte signifikant negativ mit der Trockenmasse $(-0,74)$ und dem Deckungsgrad $(-0,76)$ der Bodenvegetation der ersten Vegetationsperiode, inklusive eingesäter Nutzpflanzen. Der gesamte Höhenzuwachs des Pappelstecklings "Max 4" wurde signifikant negativ von dem Aufkommen von Lolium perenne, dem Deutschen Weidelgras, beeinflusst; der Korrelationskoeffizient nach Pearson betrug -0,73. Der Höhenzuwachs der Robinie in den ersten beiden Jahren nach der Pflanzung korrelierte signifikant negativ mit dem Deckungsgrad der gesamten Bodenvegetation $(-0,78)$, sowie mit dem Aufkommen von Lolium perenne $(-0,8)$.

\subsubsection{Nährstoffanalyse}

In diesem Kapitel werden die Nährwert-Ergebnisse der Blattanalyse, welche in der zweiten Vegetationsperiode durchgeführt wurde, dargestellt und mit in der Literatur angegeben Werten für eine ausreichende Nährstoffversorgung verglichen.

Tab 4.12: Optimale Blattspiegelwerte nach van den Burg (1990) (k.A. - keine Angaben)

\begin{tabular}{|c|c|c|c|c|c|}
\hline & $P[\mathbf{m g} / \mathrm{g}]$ & $K[\mathrm{mg} / \mathrm{g}]$ & $\begin{array}{c}\mathrm{Ca} \\
{[\mathrm{mg} / \mathrm{g}]}\end{array}$ & $\begin{array}{c}\mathrm{Mg} \\
{[\mathrm{mg} / \mathrm{g}]}\end{array}$ & $\mathbf{N}[\%]$ \\
\hline Aspe (Populus tremula) & $1,3-4,9$ & $8,0-16,0$ & $7,0-16,0$ & $1,1-4,7$ & $1,7-3,2$ \\
\hline Bergahorn (Acer pseudoplatanus) & $1,6-2,2$ & $11,0-15,0$ & k.A. & $1,6-2,7$ & $2,16-2,7$ \\
\hline Eberesche (Sorbus aucuparia) & $1,3-1,6$ & $6,0-19,1$ & 27,8 & $1,4-5,6$ & $1,8-2,2$ \\
\hline $\begin{array}{c}\text { Pappel "Max 4" } \\
\text { (P. nigra x P. maximowiczii) }\end{array}$ & k.A. & k.A. & k.A. & k.A. & 1,81 \\
\hline $\begin{array}{c}\text { Pappel "NE } 42^{\prime \prime} \\
\text { (P. maximowiczii } \times \text { P. trichocarpa) }\end{array}$ & $2,0-2,3$ & $9,0-19,0$ & 8,3 & $2,2-3,0$ & $1,68-2,6$ \\
\hline Robinie (Robinia pseudoacacia) & $1,3-11,4$ & $7,0-22,5$ & $7,7-32,7$ & $1,5-3,7$ & $2,22-5,1$ \\
\hline Schwarzerle (Alnus glutinosa) & $1,6-2,7$ & $5,4-20,0$ & $10,2-22,7$ & $1,6-5,1$ & $2,0-3,24$ \\
\hline Weide "Turbo" (Salix viminalis) & $1,0-9,5$ & $8,0-22,2$ & $4,5-14,6$ & $1,7-7,4$ & $1,7-4,22$ \\
\hline Winterlinde (Tilia cordata) & $1,5-2,0$ & $10,0-15,5$ & $13,8-17,9$ & $1,1-2,8$ & $2,0-2,86$ \\
\hline
\end{tabular}

Der in Tab 4.12 dargestellte Konzentrationsbereich wurde in der von van den Burg (1990) zusammengestellten Literatur als mittelmäßig bis gut für das Pflanzenwachstum beschrieben. Der Effekt einer Düngung auf das Wachstum war laut Literatur in diesem Bereich nur sehr gering und somit nicht lohnenswert. Geringere Nährstoff- 
konzentrationen führten zu sichtbaren Vitalitätseinbußen und/oder zu einem reduzierten Wachstum der Gehölze.

Über den Bereich einer ausreichenden Nährstoffversorgung hinaus steigende Nährstoff-Konzentrationen hatten keine positiven Auswirkungen auf das Wachstum der Gehölze - die Wachstumskurve in diesem Bereich verhielt sich konstant. Im schlimmsten Fall führte eine zu hohe Nährstoffkonzentration zu Wachstumseinbußen.

\subsubsection{Flächenvornutzung}

Insgesamt betrachtet waren alle Gehölze in der zweiten Vegetationsperiode ausreichend mit Nährstoffen versorgt, Defizite ergaben sich in Einzelfällen bei der Versorgung mit Kalium (K), Magnesium (Mg) und Stickstoff (N) (Tab. 4.13).

Tab. 4.13: Nährstoffkonzentrationen der gepflanzten Gehölze auf Acker- und Grünlandfläche in Gütersloh in der zweiten Vegetationsperiode

\begin{tabular}{|c|c|c|c|c|c|c|c|c|}
\hline & & $\begin{array}{c}P \\
{[\mathrm{mg} / \mathrm{g}]}\end{array}$ & $\begin{array}{c}K \\
{[\mathrm{mg} / \mathrm{g}]}\end{array}$ & $\begin{array}{c}\mathrm{Ca} \\
{[\mathrm{mg} / \mathrm{g}]}\end{array}$ & $\begin{array}{c}\mathrm{Mg} \\
{[\mathrm{mg} / \mathrm{g}]}\end{array}$ & $\mathbf{N}$ [\%] & $\mathbf{C}[\%]$ & $\mathbf{C} / \mathbf{N}$ \\
\hline \multirow{9}{*}{ 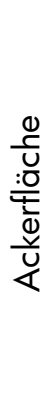 } & Aspe & 1,9 & 12,5 & 15,8 & 2,3 & 2,2 & 48,0 & 21,8 \\
\hline & Bergahorn & 6,2 & 7,8 & 23,8 & 2,4 & 1,3 & 46,7 & 37,0 \\
\hline & Eberesche & 6,9 & 8,3 & 31,9 & 2,8 & 1,8 & 47,5 & 27,4 \\
\hline & Pappel „Max 4" & 10,4 & 22,8 & 20,6 & 1,9 & 2,0 & 45,1 & 22,3 \\
\hline & Pappel "NE 42" & 4,4 & 11,5 & 19,5 & 2,0 & 1,6 & 47,2 & 30,5 \\
\hline & Robinie & 1,8 & 11,3 & 28,0 & 1,1 & 3,4 & 47,7 & 13,9 \\
\hline & Schwarzerle & 2,0 & 9,6 & 18,9 & 2,3 & 2,9 & 48,4 & 16,6 \\
\hline & Weide "Turbo" & 4,4 & 16,5 & 12,6 & 1,6 & 2,4 & 49,7 & 20,8 \\
\hline & Winterlinde & 5,4 & 15,6 & 22,4 & 1,3 & 2,4 & 47,3 & 20,2 \\
\hline \multirow{5}{*}{ 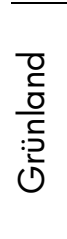 } & Aspe & 1,8 & 6,2 & 16,2 & 5,5 & 2,1 & 48,6 & 24,2 \\
\hline & Pappel "Max 4" & 4,9 & 10,2 & 16,9 & 3,7 & 1,9 & 47,2 & 25,1 \\
\hline & Pappel „NE 42" & 2,5 & 6,3 & 15,2 & 3,3 & 2,0 & 49,0 & 24,9 \\
\hline & Robinie & 1,8 & 4,3 & 25,3 & 2,5 & 3,4 & 47,6 & 14,1 \\
\hline & Weide "Turbo" & 4,5 & 11,4 & 16,1 & 3,7 & 2,4 & 48,1 & 20,1 \\
\hline
\end{tabular}

Auf der Ackerfläche war die Aspe mit nur 6,2 mg/g Kalium unzureichend versorgt, so dass sich laut van den Burg (1990) eine Düngung positiv auswirken würde. Beim Bergahorn wies die Blattanalyse auf ein Defizit in der Stickstoffversorgung (1,3\%) und der Kaliumversorgung $(7,8 \mathrm{mg} / \mathrm{g})$ hin. Die Eberesche befand sich mit einem N-Gehalt von $1,8 \%$ in der zweiten Vegetationsperiode an der unteren Grenze einer 
ausreichenden N-Versorgung. Die Pappelstecklinge "NE 42" hatten mit nur 6,3 mg/g ein Kalium-Defizit, die Versorgung mit Magnesium und Stickstoff war mit 2,0 mg/g Mg und einem N-Gehalt von 1,6 \% ebenfalls etwas gering. Die Stecklinge der Weide "Turbo" hatten laut der Blattanalyse eine leichte Unterversorgung mit Magnesium, dessen Konzentration bei $1,6 \mathrm{mg} / \mathrm{g}$ in der Blatt-Trockenmasse lag.

Auf der Grünlandfläche war die Kalium-Versorgung das Hauptproblem in Bezug auf eine ausreichende Nährstoffversorgung der Gehölze. Die Kalium-Konzentration der Aspe und des Pappelhybriden lag mit einer Konzentration 6,2 bzw. 6,3 mg/g unter dem optimalen Versorgungsbereich. Der Pappelhybrid "Max 4" hatte ebenfalls ein Kalium-Wert im unteren Bereich einer ausreichenden Kalium-Versorgung.

Auffällig war die große Diskrepanz zwischen der Kaliumversorgung auf der Ackerund der Grünlandfläche. Bei den fünf auf beiden Flächen gepflanzten Gehölzen war die Kalium-Konzentration auf der Ackerfläche etwa doppelt so hoch wie auf der Grünlandfläche. Bei den Pappelstecklingen war ebenfalls die Phosphat-Konzentration in den Pappelblättern auf der Grünlandfläche um knapp die Hälfte im Vergleich zur Ackerfläche reduziert. Und auch die Ca-Konzentration war bei den Pappelstecklingen auf der Grünlandfläche etwas verringert gegenüber der Ackerfläche.

Auf der Grünlandfläche war dagegen die Magnesium-Konzentration bei allen Gehölzen zweimal so hoch wie auf der Ackerfläche. Die N- und C-Konzentrationen bewegten sich auf den unterschiedlich vorgenutzten Teilflächen auf einem ähnlichen Niveau.

Die P-, K-, und Ca-Konzentrationen in den Pappelblättern korrelierten signifikant positiv mit den Entwicklungen der Höhe und des Wurzelhalsdurchmessers der Pappelstecklinge in den ersten beiden Jahren nach der Pflanzung. Je besser die Pappelstecklinge mit Phosphat, Kalium und Calcium versorgt wurden, desto höher war der Zuwachs des Pappelhybriden "Max 4" (Abb. 4.16) und "NE 42" (Abb. 4.17). Insbesondere die P-Konzentration zeigte einen höchstsignifikanten Einfluss $(p<0,001)$ auf den Zuwachs im zweiten Jahr sowie die Gesamthöhe und den Wurzelhalsdurchmesser nach zwei Wachstumsperioden. 


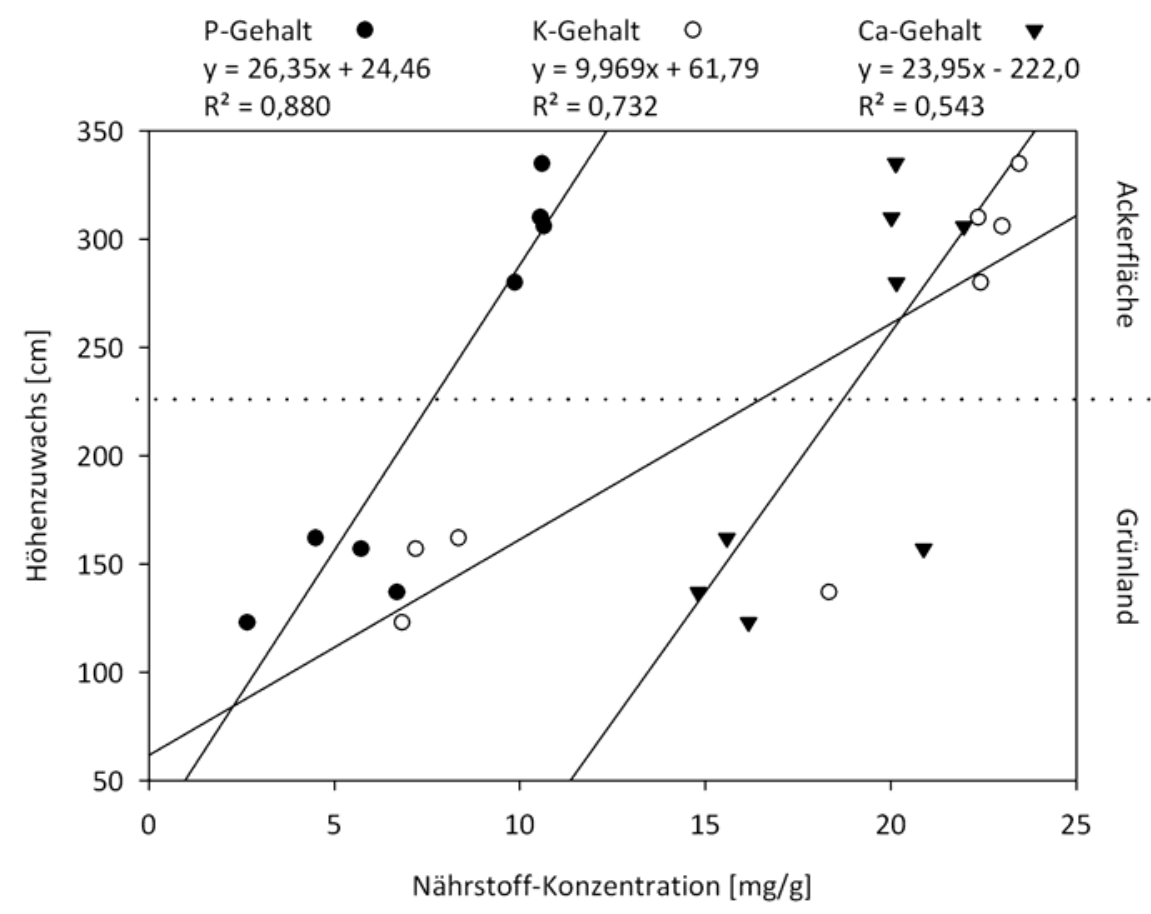

Abb. 4.16: Regression des Höhenzuwachses des 2-jährigen Pappelstecklings „Max 4“ mit der P- und K-Konzentration in der Blatt-Trockenmasse Auf der Acker- und Grünlandfläche Gütersloh

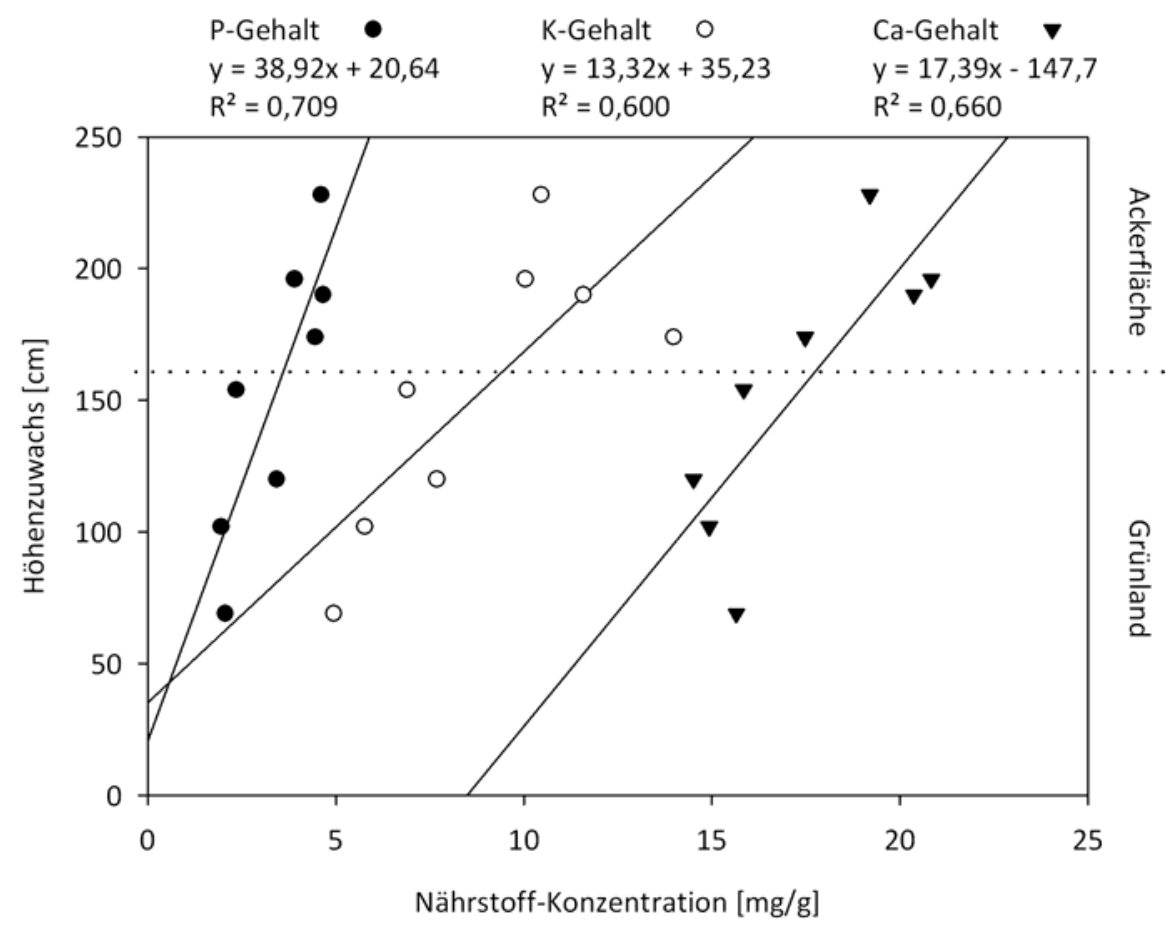

Abb. 4.17: Regression des Höhenzuwachses des 2-jährigen Pappelstecklings „NE 42“ mit den P-, K- und Ca-Konzentrationen in der Blatt-Trockenmasse auf der Acker- und Grünlandfläche in Gütersloh 
Die Zunahme der Höhe und des Wurzelhalsdurchmessers im zweiten Jahr sowie der Gesamtzuwachs an Höhe und Durchmesser der Aspe und Robinie korrelierten ebenfalls positiv mit der im zweiten Jahr gemessenen Kalium-Versorgung der Gehölze (Abb. 4. 18).

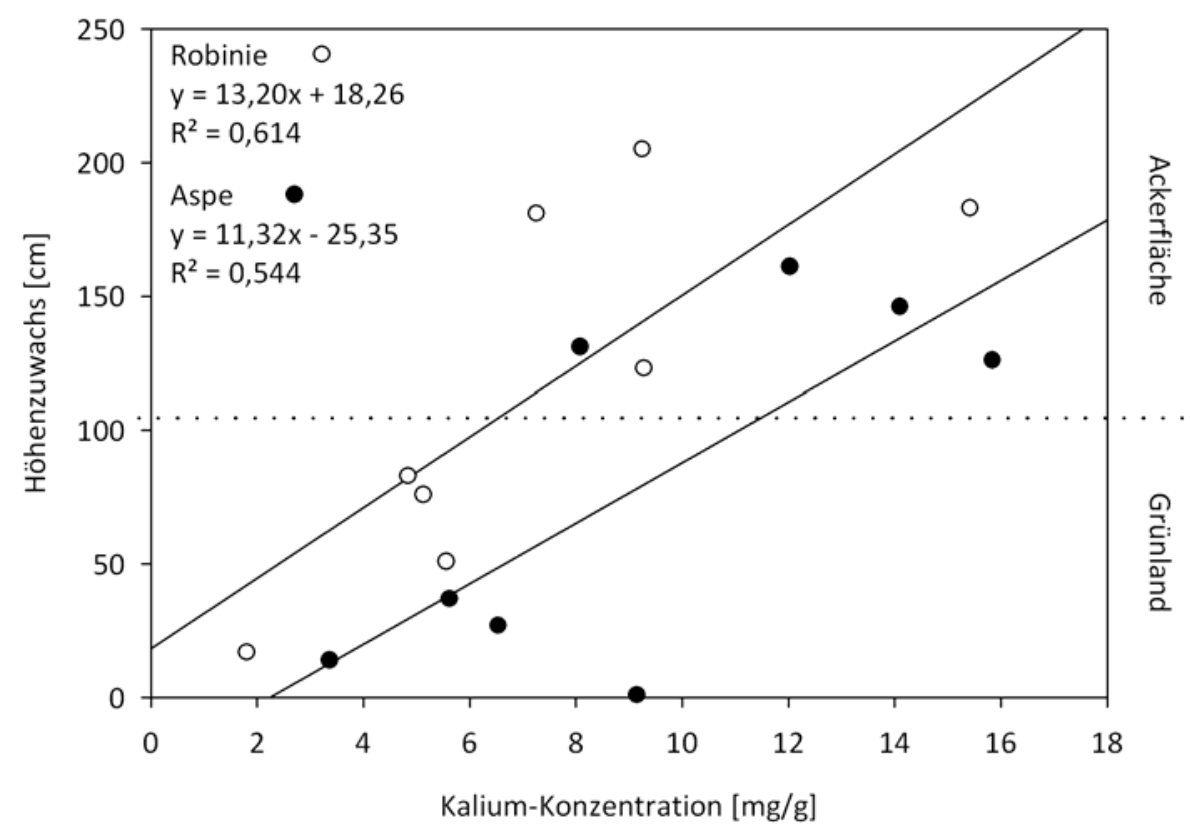

Abb. 4.18: Regression des Höhenzuwachses der Aspe und Robinie in zwei Vegetationsperioden mit der K-Konzentration in der Blatt-Trockenmasse auf der Acker- und Grünlandfläche in Gütersloh

\subsubsection{Begleitwuchsregulierung}

In Tab. 4.14 sind die Ergebnisse der ausführlicheren Blattanalyse der Baumarten Bergahorn, Pappel "Max 4" und Weide "Turbo" in der zweiten Vegetationsperiode aufgelistet. Neben dem allgemeinen Nährstoffzustand der Kontrollpflanzen sollten an den drei genannten Baumarten die Auswirkungen der Begleitwuchsregulierung (Kontrolle; Mähen; Nutzpflanzen) und der Bodenhilfsstoffe (Hydrogel; Mykorrhiza; Hydrogel + Mykorrhiza) auf die Nährstoffkonzentrationen in der Blattbiomasse untersucht werden.

Das Mähen als Begleitwuchsregulierung zeigte im Vergleich zur Kontrollfläche ohne Pflegemaßnahmen keine Auswirkungen auf die Nährstoffversorgung der Gehölze. Auffällig ist, dass die eingesäten Nutzpflanzen bei allen drei Baumarten die P. 
Konzentration signifikant absinken ließen. Neben der P-Konzentration war beim Bergahorn der N-Gehalt signifikant erhöht und bei dem Pappelhybrid "Max 4" war die Ca-Konzentration signifikant niedriger als bei den Kontrollpflanzen.

Tab. 4.14: Nährstoffkonzentrationen von Bergahorn, Pappel "Max 4" und Weide "Turbo" auf der Ackerfläche in Gütersloh in der zweiten Vegetationsperiode in Abhängigkeit von der Begleitwuchsregulierung (Mähen; Nutzpflanzen) und den applizierten Bodenhilfsstoffen (Hydrogel, Mykorrhiza; Hydrogel + Mykorrhiza) (* signifikante Mittelwert-Differenz zur Kontrolle mit $p \leq 0,05$ )

\begin{tabular}{|c|c|c|c|c|c|c|c|c|}
\hline & & $\begin{array}{c}P \\
{[\mathrm{mg} / \mathrm{g}]}\end{array}$ & $\begin{array}{c}\mathrm{K} \\
{[\mathrm{mg} / \mathrm{g}]}\end{array}$ & $\begin{array}{c}\mathrm{Ca} \\
{[\mathrm{mg} / \mathrm{g}]}\end{array}$ & $\begin{array}{c}\mathrm{Mg} \\
{[\mathrm{mg} / \mathrm{g}]}\end{array}$ & $\mathbf{N}[\%]$ & C [\%] & $\mathbf{C} / \mathbf{N}$ \\
\hline \multirow{6}{*}{$\begin{array}{l}\subseteq \\
\frac{0}{0} \\
\frac{c}{0} \\
\frac{0}{0} \\
\infty\end{array}$} & Kontrolle & 6,2 & 7,8 & 23,8 & 2,4 & 1,3 & 46,7 & 37,0 \\
\hline & Mähen & 6,5 & 9,6 & 24,3 & 3,0 & 1,5 & 46,4 & 31,4 \\
\hline & Nutzpflanzen & $2,7^{*}$ & 9,9 & 16,7 & 2,1 & $2,3^{*}$ & 47,0 & $20,8^{*}$ \\
\hline & Hydrogel & 6,5 & 6,9 & 24,3 & 3,5 & 1,3 & 46,7 & 37,0 \\
\hline & Mykorrhiza & 5,3 & 6,7 & 23,0 & 3,1 & 1,5 & 47,6 & 31,4 \\
\hline & Hydro. + Myk. & 5,4 & $6,0 *$ & 25,1 & 3,2 & 1,3 & 46,5 & 36,6 \\
\hline \multirow{6}{*}{$\begin{array}{l}\frac{x}{0} \\
= \\
\frac{0}{0} \\
\frac{0}{0} \\
\frac{0}{0}\end{array}$} & Kontrolle & 10,4 & 22,8 & 20,6 & 1,9 & 2,0 & 45,1 & 22,3 \\
\hline & Mähen & 9,9 & 23,8 & 19,1 & 2,0 & 2,0 & 45,0 & 22,7 \\
\hline & Nutzpflanzen & $7,3^{*}$ & 22,4 & $14,4^{*}$ & 1,8 & 2,2 & 45,5 & 20,7 \\
\hline & Hydrogel & $8,0 *$ & 23,6 & $17,3^{*}$ & 1,7 & 1,9 & $46,3^{*}$ & 24,0 \\
\hline & Mykorrhiza & 11,4 & 23,3 & 21,5 & $2,2^{*}$ & 2,0 & 44,9 & 22,7 \\
\hline & Hydro. + Myk. & 9,7 & 24,0 & 18,4 & 1,8 & 1,9 & 45,7 & 23,9 \\
\hline \multirow{6}{*}{$\begin{array}{l}\frac{3}{8} \\
\frac{0}{3} \\
1= \\
\frac{0}{0} \\
\frac{0}{0} \\
3\end{array}$} & Kontrolle & 4,4 & 16,5 & 12,6 & 1,6 & 2,4 & 49,7 & 20,8 \\
\hline & Mähen & 5,6 & 20,4 & 13,1 & 1,3 & 2,4 & 48,2 & 20,5 \\
\hline & Nutzpflanzen & $2,9 *$ & 14,2 & 12,4 & 1,7 & 2,4 & 48,4 & 20,4 \\
\hline & Hydrogel & 4,2 & 16,2 & $14,5^{*}$ & 1,8 & 2,2 & 48,8 & 22,3 \\
\hline & Mykorrhiza & 4,3 & 15,1 & 11,8 & 1,6 & 2,3 & 49,4 & 21,6 \\
\hline & Hydro. + Myk. & 4,7 & 16,9 & 12,2 & 1,5 & 2,3 & 48,6 & 21,6 \\
\hline
\end{tabular}

\subsubsection{Bodenhilfsstoffe}

Der bei der Pflanzung applizierte Bodenhilfsstoff Hydrogel und die Mykorrhizierung der Gehölze zeigten kaum Auswirkungen auf die Ergebnisse der Blattanalyse (Tab. 4.14). Beim Bergahorn war die K-Konzentration bei der kombinierten Anwendung von Hydrogel und Mykorrhiza im Vergleich zu den Kontrollpflanzen signifikant reduziert. Die alleinige Applikation von Hydrogel zeigte vor allem Auswirkungen auf die Nährstoffsituation des Pappelstecklings "Max 4". Sowohl die P- als auch die CaKonzentration der Pappel "Max 4" waren bei den mit Hydrogel gepflanzten Pappeln signifikant geringer als bei den Kontrollpflanzen; die C-Konzentration war dagegen 
signifikant höher. Im Gegensatz zum Pappelsteckling war die Ca-Konzentration bei den Weidenstecklingen signifikant höher, wenn Hydrogel bei der Pflanzung appliziert wurde.

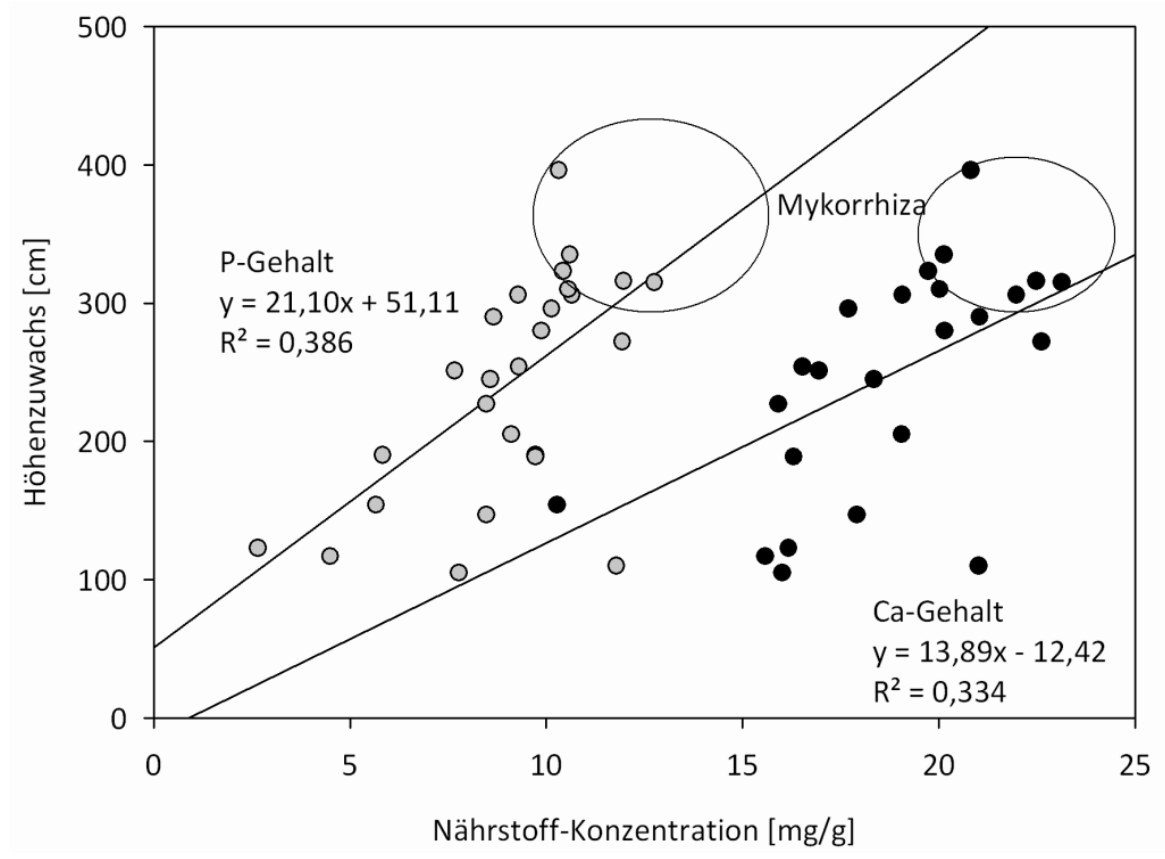

Abb. 4.19: Regression des Höhenzuwachses des mykorrhizierten und nicht mykorrhizierten Pappelstecklings "Max 4" in zwei Vegetationsperioden mit den Pund Ca-Konzentrationen in der Blatt-Trockenmasse auf der Ackerfläche in Gütersloh

Die Blattanalysen von Pappel "Max 4" und Weide "Turbo" zeigten Korrelationen zwischen einigen Nährelementen mit dem Gesamtzuwachs der Gehölze auf.

Beim Pappelsteckling "Max 4" korrelierte der Höhenzuwachs signifikant positiv mit der P- und Ca-Konzentration; der Korrelationskoeffizient nach Pearson betrug bei Phosphor 0,67 und bei Calcium 0,64. Hierbei zeigte sich ein Vorteil der mykorrhizierten Pappeln, welche die höchsten P- und Ca-Konzentrationen hatten und auch die höchsten Gesamtzuwächse nach zwei Vegetationsperioden (Abb. 4.19).

Bei Weide "Turbo" korrelierte der Höhenzuwachs signifikant mit den Nährstoffkonzentrationen von Ca und $\mathrm{Mg}$; der Korrelationskoeffizient nach Pearson lag bei 0,5 für Calcium und bei 0,47 für Magnesium. 


\subsection{Versuchsfläche Hamerstorf (Landkreis Uelzen)}

\subsubsection{Vegetationsaufnahme}

In Tab. 4.15 sind die Arten der natürlichen Begleitvegetation aufgelistet, welche maßgeblich an der Bodendeckung und der Biomassebildung der Konkurrenzvegetation verantwortlich waren. In der der zweiten Vegetationsperiode (2007) wurde die Biomasse von wesentlich weniger Arten gebildet als in der dritten Vegetationsperiode (2008). In der zweiten Vegetationsperiode waren maßgeblich 5 Arten für die Biomassebildung verantwortlich, im dritten Jahr waren es 13 verschiedene Arten, welche für die Gehölze eine Konkurrenz darstellten.

Tab. 4.15: Artenliste der biomassebildenden Begleitvegetation auf der Fläche Hamerstorf in der 2. (2007) und 3. Vegetationsperiode (2008)

\begin{tabular}{lll}
\hline Botanischer Pflanzenname & Deutscher Pflanzenname \\
\hline & Conyza canadensis & Kanadisches Berufkraut (Katzenschweif) \\
Dactylis glomerata & Gewöhnliches Knäuelgras \\
$\circ$ & Epilobium parviflorum & Kleinblütiges Weidenröschen \\
Lolium perenne & Deutsches Weidelgras \\
Poa nemoralis & Hain-Rispengras \\
\hline Apera spica-venti & Gemeiner Windhalm \\
Conyza canadensis & Kanadisches Berufkraut (Katzenschweif) \\
Dactylis glomerata & Gewöhnliches Knäuelgras \\
Elymus repens & Gewöhnliche Quecke \\
Epilobium parviflorum & Kleinblütiges Weidenröschen \\
Equisetum pratense & Wiesen-Schachtelhalm \\
Festuca pratensis & Gewöhnlicher Wiesen-Schwingel \\
Holcus lanatus & Wolliges Honiggras \\
Juncus effusus & Flatterbinse \\
Lolium perenne & Deutsches Weidelgras \\
Taraxacum officinale & Gewöhnlicher Löwenzahn \\
Phleum pratense & Wiesen-Lieschgras \\
Poa pratensis & Wiesen-Rispengras \\
\hline
\end{tabular}

Auf der Fläche in Hamerstorf zeigte sich ein signifikanter Einfluss der Bodenbearbeitung auf das Aufkommen der Bodenvegetation. Je intensiver die mechanische Bearbeitung in das Bodengefüge eingriff, desto weniger Biomasse wurde von der Begleitvegetation gebildet (Abb. 4.20). Die mit Herbiziden behandelte Fläche, auf der keine mechanische Bodenbearbeitung durchgeführt wurde, wies in der zweien 
Vegetationsperiode eine Biomassebildung von etwa $750 \mathrm{~g} / \mathrm{m}^{2}$ auf. Die Biomasse wurde hauptsächlich von Poa pratensis $\left(570 \mathrm{~g} / \mathrm{m}^{2}\right)$ und Dactylis glomerata $\left(160 \mathrm{~g} / \mathrm{m}^{2}\right)$ gebildet. Auf der geeggten Fläche wurde $550 \mathrm{~g} / \mathrm{m}^{2}$ und auf der gepflügten und geeggten Fläche nur $250 \mathrm{~g} / \mathrm{m}^{2}$ Trockenbiomasse der Bodenvegetation gebildet. Die Biomasse auf der geeggten Fläche wurde von Lolium perenne $\left(330 \mathrm{~g} / \mathrm{m}^{2}\right)$, Dactylis glomerata $\left(170 \mathrm{~g} / \mathrm{m}^{2}\right)$ und Epilobium parviflorum $\left(40 \mathrm{~g} / \mathrm{m}^{2}\right)$ gebildet, auf der gepflügten und geeggten Fläche von Conyza canadensis $\left(190 \mathrm{~g} / \mathrm{m}^{2}\right)$ und Epilobium parviflorum $\left(50 \mathrm{~g} / \mathrm{m}^{2}\right)$.

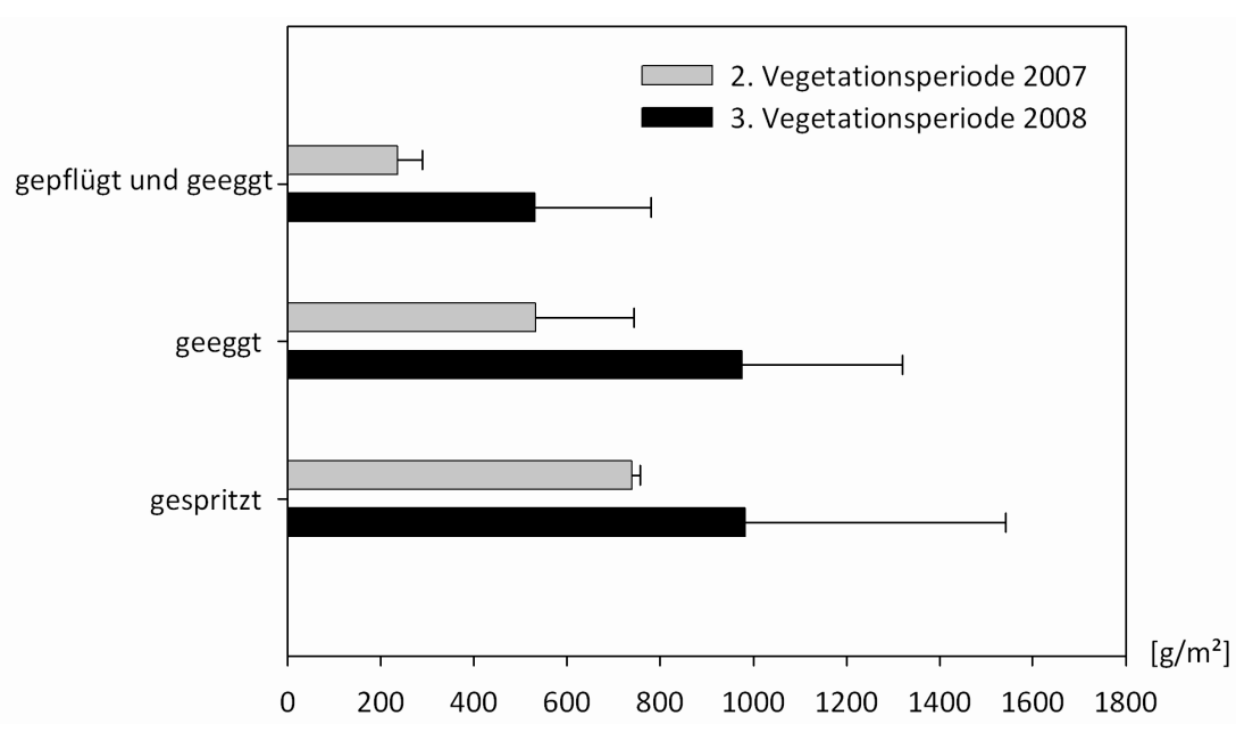

Abb. 4.20: Trockenmasse $\left(g / \mathrm{m}^{2}\right)$ der Bodenvegetation in der 2 . und 3 . Vegetationsperiode auf der Versuchsfläche Uelzen in Abhängigkeit von der Flächenvorbereitung

Sowohl der Deckungsgrad als auch die von der Bodenvegetation gebildete Biomasse war in der dritten Vegetationsperiode größer als in der zweiten. Die Methode des Pflügen und Eggens sorgte mit etwa $550 \mathrm{~g} / \mathrm{m}^{2} \mathrm{TM}$ im dritten Jahr für die geringste interspezifische Konkurrenz der Begleitvegetation mit den schnellwachsenden Baumarten. Hier waren Dactylis glomerata $\left(230 \mathrm{~g} / \mathrm{m}^{2}\right)$, Festuca pratensis $\left(140 \mathrm{~g} / \mathrm{m}^{2}\right)$ und Apera spica-venti $\left(80 \mathrm{~g} / \mathrm{m}^{2}\right)$ die dominierenden Grasarten. Auf der gespritzten und der geeggten Fläche wurde mit jeweils $1000 \mathrm{~g} / \mathrm{m}^{2}$ fast doppelt so viel Trockenmasse von der Bodenvegetation produziert. Die mit Herbiziden vorbehandelte 
Teilfläche wurde von Juncus effusus $\left(590 \mathrm{~g} / \mathrm{m}^{2}\right)$, Festuca pratensis $\left(160 \mathrm{~g} / \mathrm{m}^{2}\right)$ und Equisetum pratense $\left(90 \mathrm{~g} / \mathrm{m}^{2}\right)$ beherrscht. Auf der geeggten Fläche herrschten Juncus effusus $\left(660 \mathrm{~g} / \mathrm{m}^{2}\right)$ und Dactylis glomerata $\left(110 \mathrm{~g} / \mathrm{m}^{2}\right)$ vor

\subsection{2 Überlebensraten}

In Abb. 4.21 sind die Überlebensraten der Pappelhybriden "Androscoggin", "Max 4", "NE 42" und „Weser 6" nach der zweiten und dritten Vegetationsperiode dargestellt. Alle vier Pappelhybriden wurden auf der Teilfläche angebaut, welche vor der Kulturanlage als Flächenvorbereitung geeggt wurde; also der Teilfläche, auf der das Begleitwuchsaufkommen mittelstark im Vergleich mit den anderen Flächenvorbereitungen war.

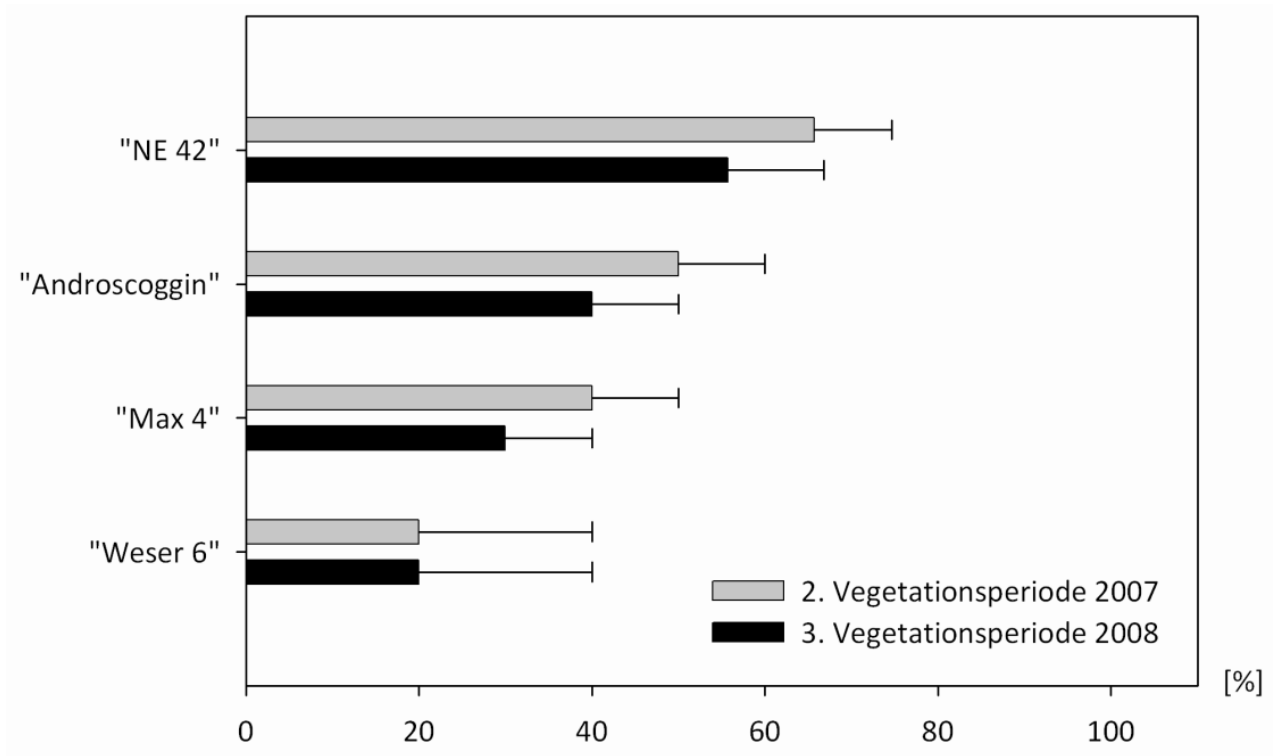

Abb. 4.21: Überlebensraten der unterschiedlichen Pappelhybriden auf der geeggten Versuchsfläche Hamerstorf nach der 2. und 3. Vegetationsperiode

Der Pappelhybrid "NE 42" wies die höchsten Überlebensraten auf der geeggten Teilfläche auf (Abb. 4.21). Nach der zweiten Vegetationsperiode betrug die Überlebensrate 65 \% und nach der dritten noch 55 \%. Von den Pappelhybriden 
"Androscoggin" überlebten 50 \% die zweite und $40 \%$ die dritte Vegetationsperiode. Von dem Pappelhybrid "Max 4" waren noch 40 \% nach der zweiten und nur noch 30 \% nach der dritten Vegetationsperiode am Leben. Der Pappelhybrid "Weser 6" wies die geringste Überlebensrate auf der geeggten Teilfläche auf; nur $20 \%$ der Pappel "Weser 6" erreichten das Ende der zweiten und dritten Vegetationsperiode. Nach der zweiten Vegetationsperiode unterschied sich die Überlebensrate von „Weser 6 " signifikant von der Überlebensrate der Pappel "Ne 42", ansonsten waren die Unterschiede zwischen den Pappelhybriden nicht signifikant.

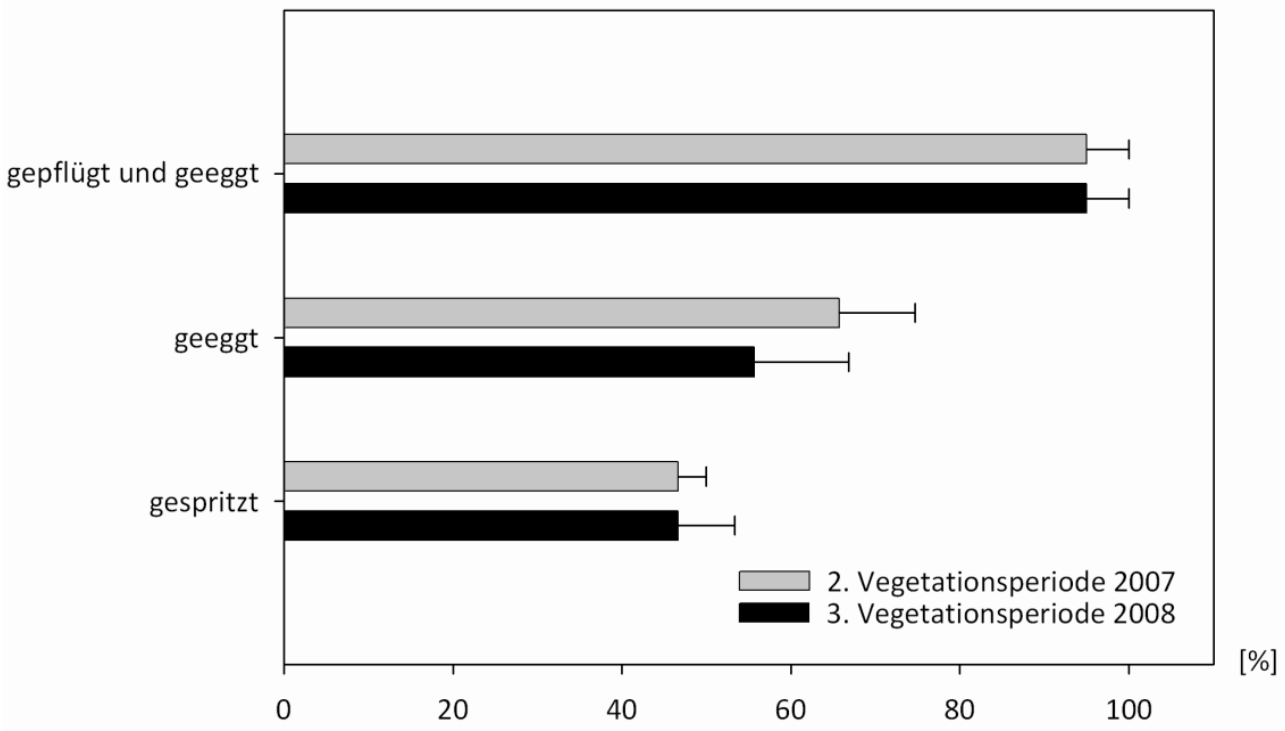

Abb. 4.22: Überlebensrate des Pappelhybriden „NE 42" nach der 2. und 3. Vegetationsperiode in Abhängigkeit von der Flächenvorbereitung auf der Versuchsfläche Hamerstorf

Der Pappelhybrid "NE 42" wurde auf allen drei unterschiedlich vorbereiteten Teilflächen (gepflügt und geeggt; geeggt; gespritzt) angebaut. Je intensiver die mechanische Flächenvorbereitung war, desto höher war die Überlebensrate der Pappel „NE 42" (Abb. 4.22). Die höchste Überlebensrate wurde auf der Teilfläche erreicht, die vor der Pflanzung gepflügt und geeggt wurde; $95 \%$ des Pappelhybriden "NE 42" waren nach der zweiten und dritten Vegetationsperiode noch am Leben. Auf der Teilfläche, die als Flächenvorbereitung nur geeggt wurde, betrug die Überlebensrate 65 \% nach der zweiten und 55 \% nach der dritten Vegetationsperiode. 
Die geringste Überlebensrate der Pappel „NE 42" war auf der gespritzten Teilfläche, auf der keine mechanische Bodenbearbeitung durchgeführt wurde, zu verzeichnen; nur $46 \%$ des Pappelstecklings "NE 42" hatten die zweite und dritte Vegetationsperiode überlebt. Die Überlebensraten auf der gespritzten Teilfläche waren signifikant geringer als auf der gepflügten und geeggten Teilfläche.

\subsubsection{Zuwachsmessungen}

Die Höhenmessungen der Pappelhybriden auf der geeggten Teilfläche, auf der alle vier Hybriden gepflanzt wurden, zeigten zum Teil erhebliche Unterschiede in den Endhöhen der Pappeln (Abb. 4.23). Nach der zweiten Vegetationsperiode konnte beim Pappelhybrid "NE 42" die größte Höhe mit 100 cm festgestellt werden. Die Pappel "Androscoggin" war mit nur $55 \mathrm{~cm}$ nur halb so groß wie "NE 42". "Max 4" erreichte nach der zweiten Vegetationsperiode eine Höhe von $65 \mathrm{~cm}$. Die Pappel "Weser 6" war mit einer Höhe von nur $40 \mathrm{~cm}$ signifikant kleiner als "NE 42".

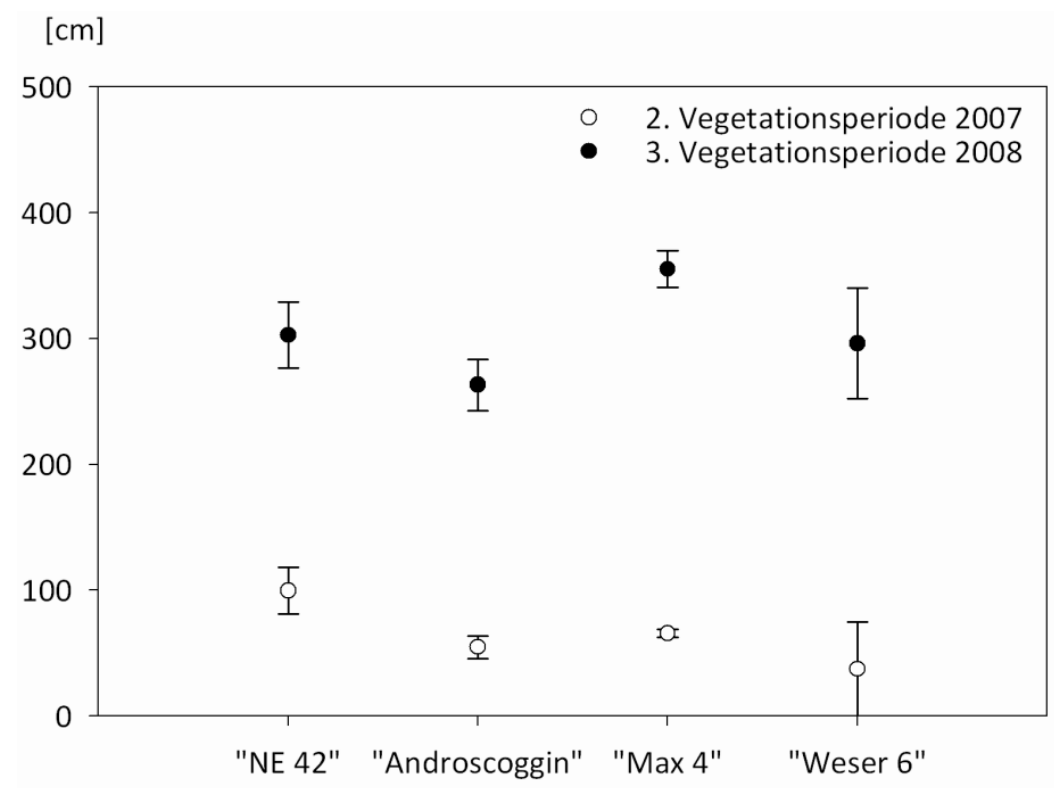

Abb. 4.23: Pappelhöhen nach der 2. und 3. Vegetationsperiode auf der geeggten Versuchsfläche Hamerstorf 
In der dritten Vegetationsperiode legten alle vier Pappelsorten erheblich an Höhe zu, mehr als in den ersten beiden Jahren zusammen. Dabei veränderte sich die Rangfolge der Pappeln in Bezug auf die Höhenentwicklung. Die Pappel "NE 42" erreichte nach dem dritten Standjahr eine Höhe von $300 \mathrm{~cm}$ und war damit dreimal so viel gewachsen wie in den ersten beiden Jahren. Die Pappel "Androscoggin" war mit $265 \mathrm{~cm}$ nach dem dritten Jahr kleiner als die anderen drei Pappelhybriden. Die größte Höhe nach der dritten Vegetationsperiode hatte der Pappelhybride "Max 4" mit $355 \mathrm{~cm}$. "Androscoggin" und "Max 4" hatten im dritten Jahr etwa das Fünffache an Höhe zugelegt. Der Höhenzuwachs von "Weser 6" war achtmal so hoch wie in den ersten beiden Jahren. "Weser 6" erreichte wie "NE 42" eine Höhe von 300 cm. Die Höhenunterschiede zwischen den Pappelhybriden waren nach dem dritten Standjahr nicht signifikant.

Auf der Fläche Hamerstorf zeigte sich ein signifikanter Einfluss der Flächenbearbeitung auf die Zuwachsentwicklung des Pappelhybriden "NE 42" in den ersten beiden Standjahren (Abb. 4.24). Je intensiver die mechanische Flächenbearbeitung vor der Pflanzung war, desto größer war der Höhenzuwachs des Pappelhybriden "NE 42".

Auf der gepflügten und geeggten Teilfläche betrug die Höhe nach der zweiten Vegetationsperiode $170 \mathrm{~cm}$. Auf der Teilfläche, die nur geeggt wurde, erreichte "NE 42" eine signifikant niedrigere Höhe von $100 \mathrm{~cm}$. Auf der gespritzten Teilfläche ohne mechanische Bodenbearbeitung hatte "NE 42" eine nur halb so große Höhe von $50 \mathrm{~cm}$, die signifikant geringer war als auf den beiden Teilflächen mit mechanischer Bodenbearbeitung.

Nach der dritten Vegetationsperiode erreichte "NE 42" eine Höhe von $375 \mathrm{~cm}$ auf der gepflügten und geeggten Teilfläche. Die Endhöhe auf der nur geeggten Teilfläche betrug $300 \mathrm{~cm}$, unterschied sich aber nicht signifikant von der Pappelhöhe auf der gepflügten und geeggten Teilfläche. Die geringste Höhe wurde auf der gespritzten Teilfläche gemessen; die Höhe von "NE 42" betrug hier $200 \mathrm{~cm}$ und war signifikant geringer als auf den beiden anderen Teilflächen. 


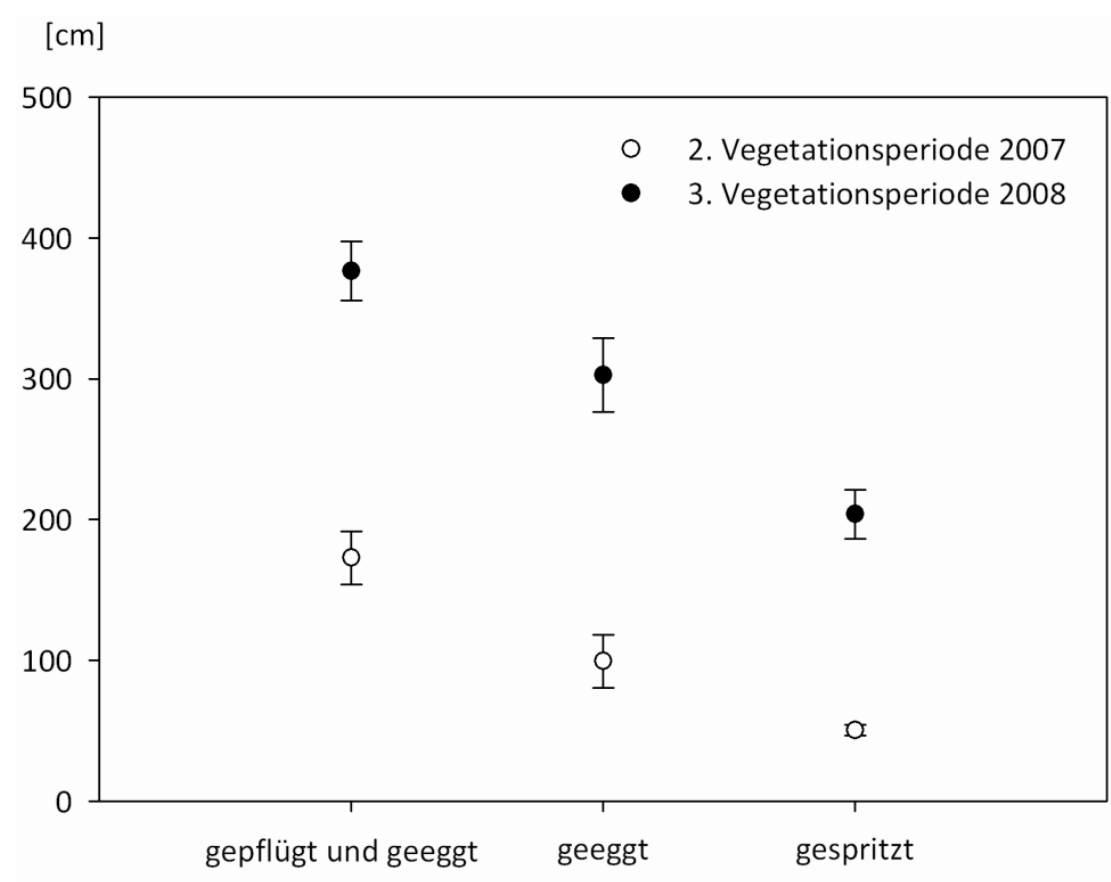

Abb. 4.24: Höhe des Pappelhybriden „Ne 42" nach der 2. und 3. Vegetationsperiode in Abhängigkeit von der Vorbereitung der Versuchsfläche Hamerstorf

\subsection{Versuchsfläche Unter/üß (Landkreis Uelzen)}

\subsubsection{Vegetationsaufnahme}

Die Vegetationsaufnahme in Unterlüß erfolgte in der ersten Vegetationsperiode, in der die unterschiedlichen Pappelhybriden auf dem Wildacker gesteckt wurden. Die Fläche wurde vor der Pflanzung $30 \mathrm{~cm}$ tief gepflügt, ansonsten wurden keine Maßnahmen zur Begleitwuchsregulierung durchgeführt. In Tab. 4.16 sind die Hauptarten der natürlichen Bodenvegetation aufgelistet, welche mit den Pappelsteckligen um Licht und Nährstoffe konkurrierten.

Die Begleitvegetation hatte einen durchschnittlichen Deckungsgrad von $82 \%$ und bildete $274 \mathrm{~g} / \mathrm{m}^{2}$ Trockenbiomasse in der ersten Vegetationsperiode. Das ist etwas mehr als auf der gepflügten Ackerfläche in Gütersloh, auf der etwa $200 \mathrm{~g} / \mathrm{m}^{2}$ Trockenbiomasse von der natürlichen Begleitvegetation gebildet wurden. 
Tab. 4.16: Artenliste der biomassebildenden Begleitvegetation auf der Versuchsfläche Unterlüß in der ersten Vegetationsperiode 2007 (DG-Deckungsgrad; TM Trockenmasse; \pm Standardabweichung Trockenmasse)

\begin{tabular}{llccc}
\hline $\begin{array}{l}\text { Botanischer } \\
\text { Pflanzenname }\end{array}$ & $\begin{array}{l}\text { Deutscher } \\
\text { Pflanzenname }\end{array}$ & DG [\%] & TM [g/ $\left.\mathbf{m}^{2}\right]$ & $\mathbf{\pm}$ \\
\hline Achillea millefolium & Gemeine Schafgarbe & 8 & 41 & 90,7 \\
Chenopodium album & Weißer Gänsefuß & 15 & 73 & 103,0 \\
Dactylis glomerata & Gewöhnliches Knäuelgras & 14 & 56 & 67,0 \\
Lolium perenne & Deutsches Weidelgras & 14 & 33 & 36,1 \\
Rumex acetosella & Kleiner Sauerampfer & 14 & 27 & 26,3 \\
Tripleurospermum maritimum & Geruchlose Kamille & 15 & 42 & 39,8 \\
Viola arvensis & Acker-Stiefmütterchen & 2 & 2 & 4,3 \\
\hline Summe & & 82 & 274 & 93,1 \\
\hline
\end{tabular}

\subsubsection{Anwuchsverhalten}

In Abb. 4.25 sind die prozentualen Anwuchsraten der Pappelhybriden "NE 42", "Max 3" und "Weser 6" auf der Fläche Unterlüß Abt.229 in Abhängigkeit von ihrer Mykorrhizierung dargestellt.

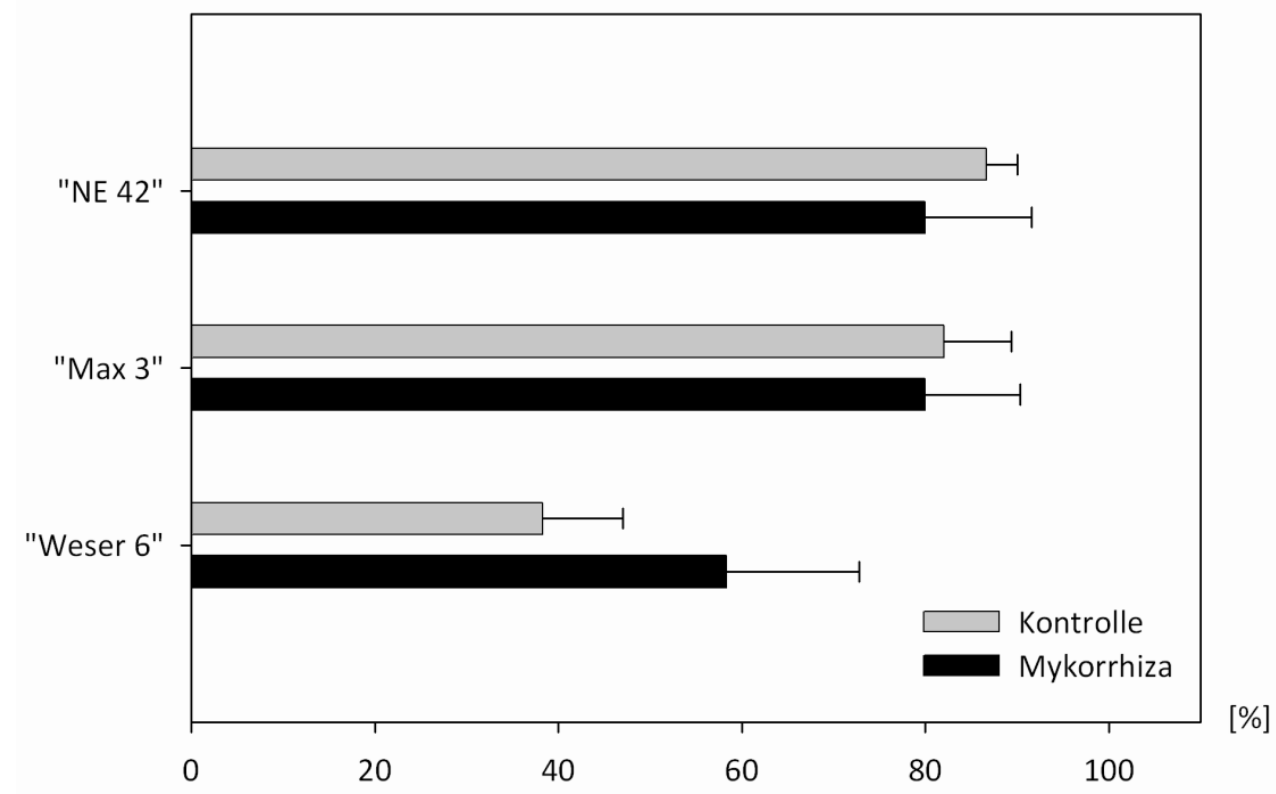

Abb. 4.25: Anwuchsraten der Pappelhybride in der ersten Vegetationsperiode auf dem Wildacker in Unterlüß in Abhängigkeit von einer Mykorrhizierung 
Bei den Kontrollpflanzen, die nicht mykorrhiziert wurden, wiesen "NE 42" und "Max 3" Anwuchsraten um die 85 \% auf. Vom Pappelhybrid "Weser 6" trieben nur halb so viele Stecklinge aus; die Anwuchsrate lag bei $40 \%$ und war damit signifikant geringer als bei "NE 42" und "Max 3".

Die mykorrhizierten Stecklinge der Pappelhybriden "NE 42" und "Max 3" hatten mit $80 \%$ eine ebenso hohe Anwuchsrate wie die Kontrollpflanzen. Die Mykorrhizierung erhöhte den Anwuchs des Pappelhybrids "Weser 6" auf 60 \%. Der Unterschied der Anwuchsrate zwischen mykorrhizierten und nicht mykorrhizierten Stecklingen war nicht signifikant. Auch der Unterschied zwischen den Anwuchsraten des Pappelhybrids „Weser 6" und der Pappelhybride "NE 42" und "Max 3" war bei den mykorrhizierten Stecklingen nicht signifikant.

\subsubsection{Zuwächse}

Die Höhenzuwächse lagen in der ersten Vegetationsperiode zwischen 25 und $45 \mathrm{~cm}$ (Abb. 4.26).

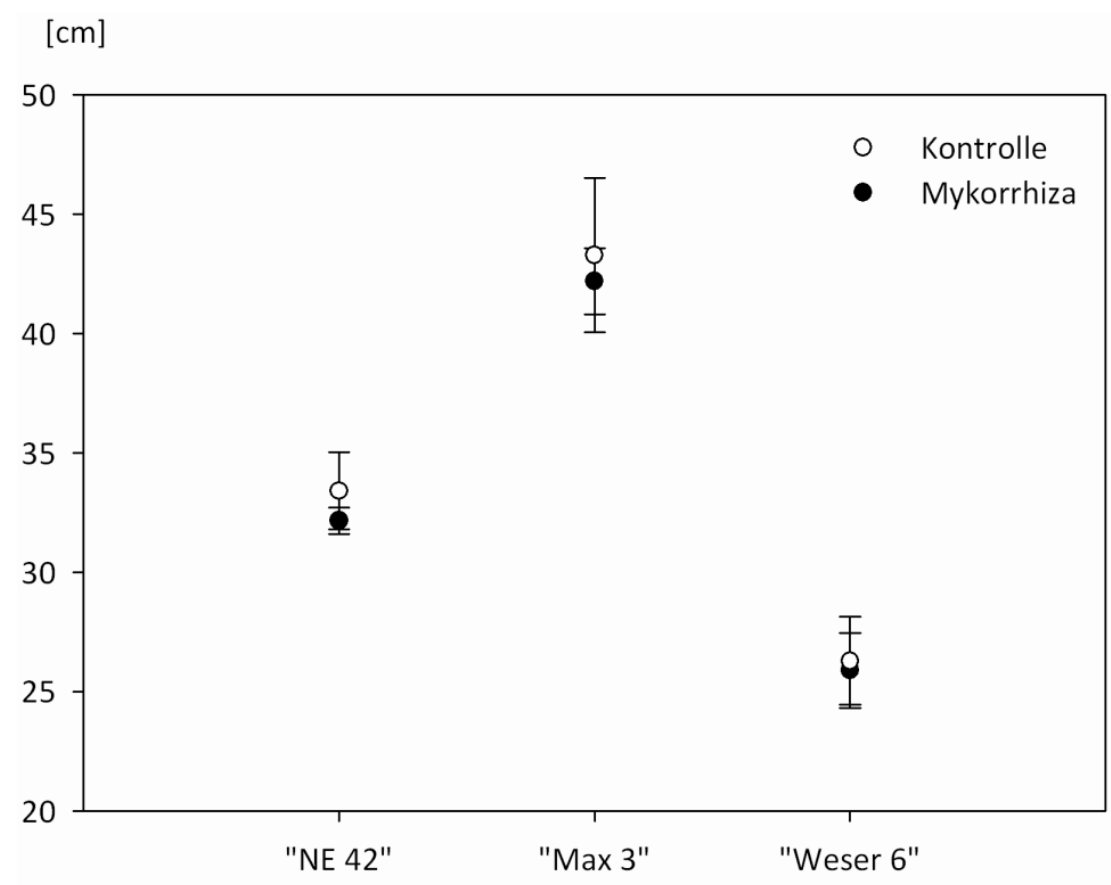

Abb. 4.26: Höhe der Pappelhybriden auf dem Wildacker in Unterlüß nach der ersten Vegetationsperiode in Abhängigkeit von einer Mykorrhizierung 
Die Pflanzen des Pappelhybriden "NE 42" wuchsen im ersten Jahr durchschnittlich $32 \mathrm{~cm}$. Der Pappelhybrid "Max 3" erreichte mit $42 \mathrm{~cm}$ die größte Wuchshöhe von den gepflanzten Pappeln und war signifikant höher als die Pappelklone "NE 42" und „Weser 6". Der geringste Höhenzuwachs wurde von dem Pappelhybrid "Weser 6" erreicht; "Weser 6 hatte eine Höhe von 26 cm nach der ersten Vegetationsperiode.

Bei allen drei Pappelhybriden war kein nennenswerter Wuchsunterschied zwischen den mykorrhizierten und nicht mykorrhizierten Stecklingen messbar. Allerdings war der Höhenunterschied zwischen allen drei mykorrhizierten Pappelhybriden statistisch signifikant. 


\section{Diskussion}

\subsection{Baumartenwahl}

Auf der ehemaligen Ackerfläche in Gütersloh wiesen die gepflanzten Baumarten eine Anwuchsrate von 80-100 \% auf. Ausnahme war die Schwarzerle, deren Anwuchsrate nur $50 \%$ betrug. Die hohe Ausfallrate der Schwarzerle ist mit den sehr trockenen Anfangsbedingungen nach der Pflanzung zu erklären. Im April 2007 fiel kaum Niederschlag. Die Niederschlagssumme betrug nicht mal $10 \%$ des langiährigen Mittels mit um 60 \% höheren Temperaturen. Diese extremen Anfangsbedingungen führten bei der gerade gepflanzten Schwarzerle zu besonders hohen Ausfällen. Nach Eschenbach und Kappen (1999) weist die Schwarzerle unter den heimischen Baumarten die höchsten Transpirationsraten auf, weshalb sie auf Trockenheit sehr empfindlich reagiert.

Die Robinie wies nach einem guten Anwuchs hohe Ausfallraten nach der ersten Überwinterung auf der Fläche auf. Die 20 \% der gepflanzten Robinien fielen den Wühlmäusen, insbesondere Erdmäusen, zum Opfer. Die Robinie wies im Vergleich mit den anderen Baumarten die meisten durch Mäusefraß verursachten Ausfälle auf. Auch die Pappelstecklinge und Schwarzerle gehörten mit etwa 15 \% Ausfälle durch Mäuse zu den favorisierten Nahrungsquellen der Mäusepopulation. Verkrautete und vergraste Standorte wie Erstaufforstungsflächen stellen günstige Lebensräume für Mäuse dar, in denen die Nager vor Feinden geschützt die Wurzeln und Wurzelhalsbereich der jungen Gehölze abnagen und damit hohe Ausfälle herbeiführen können (Stanturf et al., 2001). Robinie gehört ebenso wie Ahorn, Pappel und Weide zu den durch Erdmausfraß stark gefährdeten Baumarten (Wenk, 2007). Insgesamt wurden etwa 30 \% der Pflanzenausfälle auf der Versuchsfläche Sudheide durch Wühlmausschäden verursacht; $12 \%$ der gepflanzten Gehölze. Bei Massenvermehrungen von Wühlmäusen kann der Großteil der gepflanzten Bäume geschält werden, von diesen fallen 10-50 \% aus (Odermatt \& Wasem, 2004). Bei Don et al. (2007) wurden bis zu 20 \% der Ausfälle einer Baumart auf Schermausschäden zurückgeführt, wobei die 
Baumarten unterschiedlich stark durch diese Schäden betroffen waren. Durch die Pflanzung artenreicher Bestände konnten die Verbissschäden durch Wühlmäuse im Vergleich zu artenarmen Beständen verringert werden.

Auf der Grünlandfläche in Gütersloh waren die Anwuchsraten der Gehölze geringer als auf der Ackerfläche. Die Pappelstecklinge wiesen auf der Ackerfläche Anwuchsraten von mindestens 80 \% auf, was laut Wolf und Böhnisch (2003) Anwuchsraten auf guten Standorten (Bodenwertzahl ca. 65) entspricht. Auf der Grünlandfläche lagen die Anwuchsraten bei nur 30-70 \%. Ähnliche Anwuchszahlen von Pappelklonen erwähnen auch Wolf und Böhnisch (2003) in ihrem Anbauversuch auf landwirtschaftlichen Flächen. Auf schwächeren Standorten (Bodenwertzahlen um die 40) bewegte sich der Anwuchs der angebauten Klone bei 30-90\%. Bei Pappelstecklingen bestehen Unterschiede bezüglich der Anwuchsraten zwischen den Klonen. "Max 4" wies auf der Grünlandfläche in Gütersloh eine annähernd doppelt so hohe Überlebensrate wie "NE 42" auf. Die Ausfälle ereigneten sich dabei hauptsächlich im ersten Wuchsjahr nach Einbringung der Steckhölzer. Nach Etablierung des Wurzelsystems und des oberirdischen Sprosses waren die Ausfallraten wesentlich geringer. Auch auf der Fläche Hamerstorf in Uelzen war der Unterschied zwischen den Anwuchsraten den Pappelsorten sehr groß; die Überlebensraten bewegten sich bei 20-70\%. Hier schnitt "NE 42" besser ab als die Pappelstecklinge von "Androscoggin", Max 4" und "Weser 6".

Auch bei den Höhenzuwächsen ergaben sich deutliche Unterschiede zwischen den Baumarten, wobei die Höhenzuwächse auf der Grünlandfläche im Vergleich zur Ackerfläche durchschnittlich um die Hälfte geringer waren. Wie erwartet wurden die höchsten Zuwächse von den Weiden- und Pappelstecklingen mit 2-3 m nach zwei Vegetationsperioden erbracht. Auf der Versuchsfläche Hamerstorf erreichten die Pappelstecklinge eine Wuchshöhe von etwa $3 \mathrm{~m}$ nach drei Vegetationsperioden. Dies entspricht annähernd der von Röhricht und Ruscher (2004) gemessenen mittleren Wuchshöhe für Korbweide und den Pappelhybrid "Max 3" von 2,50 m nach zwei Vegetationsperioden auf einem sandigen Lehmboden (Ackerzahl 59). Stanturf et al. 
(2001) geben für Pappelhybriden in Nordamerika ein durchschnittliches jährliches Höhenwachstum von 1,00-3,40 m in den ersten 3-4 Jahren nach der Pflanzung an. Das Wachstumspotential von Pappelhybriden ist abhängig von den Standortverhältnissen, der Sorte und der Pflegeintensität (Stanturf et al., 2001).

Aspe und Robinie hatten mit 1-2 m nach zwei Standjahren etwas geringere Höhenzuwächse als Weide und Pappel. Der Höhenzuwachs der Aspe war deutlich höher als bei Don et al. (2007), bei denen die vitalen Pflanzen Zuwächse von etwa 40 cm in zwei Vegetationsperioden hatten, und geringer als bei Yu et al. (2001), bei denen die Aspe $140 \mathrm{~cm}$ in zwei Jahren wuchs. Eisenreich (1956) spricht bei durchschnittlichen jährlichen Höhenzuwächsen von etwa $50-100 \mathrm{~cm}$ von äußerst günstigen Wuchsleistungen der Aspe. Das bedeutet, dass die Aspe in der vorliegenden Arbeit mit durchschnittlich $55 \mathrm{~cm}$ Höhenzuwachs pro Jahr eine gute Wuchsleistung erreicht hat. Hybrid-Aspen hatten bei Reisner (2001) trotz Trockenheit eine Überlebensrate von 90 \% und einen durchschnittlichen Höhenzuwachs von 30 $\mathrm{cm}$, mit einem Maximum von über $80 \mathrm{~cm}$. Bei Yu et al. (2001) waren Hybrid-Aspen (Populus tremula $\times$ Populus tremuloides) wesentlich wuchskräftiger als herkömmliche Aspen und erreichten fast 4-mal höhere Stammvolumina als Aspen lokaler Herkunft. Der jährliche Höhenzuwachs der Robinie wird von der Landesanstalt für Ökologie, Landschaftsentwicklung und Forstplanung NRW (1984) mit 0,45-1,20 m in den ersten 10 Jahren angegeben. Auf der Ackerfläche Sudheide wurde das Wuchspotenzial der Robinie somit zu zwei Drittel ausgeschöpft.

Aber auch die für Kurzumtriebsplantagen eher ungewöhnlichen Baumarten Bergahorn Eberesche, Schwarzerle und Winterlinde hatten mit 30-80 cm Höhenzuwachs in zwei Jahren nach der Pflanzung noch gute Wuchsleistungen vorzuweisen. Die Zuwächse von Bergahorn, Eberesche und Winterlinde lagen deutlich über den Zuwächsen des Anbauversuchs von Don et al. (2007), die in zwei Vegetationsperioden Höhenzuwächse von 10-30 cm gemessen haben. Auf bayerischen Probeflächen erreichten Bergahorne im Alter von 5-10 Jahren einen Jahreszuwachs von $<15 \mathrm{~cm}$ auf Mangelstandorten und bis $>60 \mathrm{~cm}$ auf Optimalstandorten (Weber \& Bahr, 2000). In 
der vorliegenden Arbeit wurde bei dem vieriährigen Bergahorn ein durchschnittlicher iährlicher Höhenzuwachs von etwa $30 \mathrm{~cm}$ gemessen, was bei Weber und Bahr (2000) dem Zuwachs auf Grenzbereichsstandorten entspricht. Von Eisenreich (1956) wurden für Ebereschen jährliche Wuchsleistungen von $30-50 \mathrm{~cm}$ zusammengetragen, welche die Eberesche in dieser Arbeit ebenfalls erreicht. Schwarzerlen können unter günstigen Bedingungen bereits nach drei Jahren Höhen von über $2 \mathrm{~m}$ erreichen (Eisenreich, 1956). Aufgrund der für Schwarzerlen nicht ganz optimalen Standortbedingungen reichte die Wuchsleistung der Schwarzerle in dieser Arbeit nicht an diese Werte heran. Aber mit einem durchschnittlichen jährlichen Höhenzuwachs von $45 \mathrm{~cm}$ erbrachte die Schwarzerle auch auf dem weniger feuchten Boden gute Wuchsleistungen (Eisenreich, 1956). Hinzu kommt die Fähigkeit der Schwarzerle als bodenbessernde Holzart den freien Stickstoff der Luft zu binden (Eisenreich, 1956).

\subsection{Flächenvornutzung}

Die höheren Ausfallraten und geringeren Zuwächse der Gehölze auf der Grünlandfläche als auf der Ackerfläche sind durch den hohen Konkurrenzdruck der Begleitvegetation zu erklären. Die Konkurrenz der Feldvegetation beeinflusst die Ausfallhäufigkeit und Wuchskraft von Gehölzen aufgrund ihrer starken Konkurrenzkraft um Wasser und Nährstoffe signifikant (Jylhä et al., 2006; Löf \& Welander, 2004). Außerdem können Stecklinge innerhalb kürzester Zeit überwachsen und ausgedunkelt werden (Schildbach et al., 2008).

Laut Jylhä et al. (2006) ist die Konkurrenzwirkung der Unkräuter auf die gepflanzten Gehölze erst ab einen Deckungsgrad über 60 \% deutlich spürbar. Wobei die Intensität der natürlichen Vegetation stark von der Flächenvornutzung abhängt. Wird die zu begründende Fläche bis zur Anlage der Energieholzplantage als Ackerfläche genutzt, ist die Konkurrenzvegetation bereits stark zurückgedrängt, während sich bei einer Fläche, die schon einige Jahre stillgelegt ist, die Situation meist anders darstellt (Pallast et al., 2006). Die Ausfallhäufigkeit ist aber nicht allein durch den Deckungsgrad zu erklären, der in der vorliegenden Arbeit bei 60-120 \% lag. Der Anwuchs wurde 
deutlich von der Artzusammensetzung der Begleitvegetation bestimmt, ebenso wie der Zuwachs von der Intensität und der Artenzusammensetzung der Konkurrenzvegetation beeinflusst wird (Block et al., 2009). Die Anwuchsraten der Pappelstecklinge korrelierten signifikant negativ mit dem Auftreten von Lolium perenne, dem Deutschen Weidelgras.

Der Höhenzuwachs der Gehölze war in der vorliegenden Arbeit auf der von Gräsern dominierten Grünlandfläche nur halb so hoch wie auf der Ackerfläche. Gräser werden aufgrund ihrer Konkurrenzkraft um Nährstoffe und Wasser für das Wachstum junger Bäume als besonders schädlich betrachtet. Block et al. (2009) hat den Wachstumsunterschied von Pappelhybriden auf einer ehemaligen Alfalfa-Fläche und einer Weidenfläche dem höheren Grad an Konkurrenzvegetation auf der Weidenfläche zugeschrieben. Auf der Alfalfa-Fläche trat stellenweise eine flach wurzelnde Begleitvegetation auf, während die Grünfläche mit zum Teil tief wurzelnden Gräsern und Kräutern extensiv bewachsen war, welche die durchschnittliche Höhe der Pappeln reduzierten (Block et al., 2009). Bestimmte Gräser und Kräuter der Bodenvegetation können konkurrenzkräftiger als Pappelstecklinge, in Bezug auf Wasser, Nährstoffe und Licht, sein (Stanturf et al., 2001). Bei Landhäusser und Lieffers (1998) reduzierte Calamagrostis canadensis das durchschnittliche Höhenwachstum von Populus tremuloides signifikant von $49 \mathrm{~cm}$ auf $27 \mathrm{~cm}$. In der vorliegenden Arbeit beeinflusste vor eine allem aus Gräsern bestehende Konkurrenzvegetation den Höhenzuwachs der Gehölze in der ersten Vegetationsperiode negativ, was in einem reduzierten Gesamthöhenzuwachs nach zwei Vegetationsperioden nach der Pflanzung widerspiegelte. Insbesondere das Deutsche Weidelgras hatte einen negativen Effekt auf die Wuchsleistungen der Gehölze. Bei Gakis et al. (2004) war das Deutsche Weidelgras ein limitierender Faktor für das Wachstum von Bergahorn; eine signifikanter Höhenunterschied zwischen den Pflanzen auf der mit Weidelgras eingesäten Fläche und der Kontrollfläche zeigte sich dabei im dritten Jahr nach der Pflanzung, der Einfluss auf den Durchmesser war bereits im zweiten Jahr signifikant. 
Auch Minotta et al. (2001) stellten fest, dass eine Grasdecke bestehend aus Lolium perenne, Dactylis glomerata und Bromus hordeaceus die Wuchsentwicklung von Gehölzen signifikant reduzierte. Vitale Grasdecken können auf effiziente Weise den Wassergehalt aus dem Bodens extrahieren und somit die Bodenwasserspannung deutlich erhöhen (Coll et al., 2004; Davies, 1985), was insbesondere bei Wasserstress die Pflanzenvitalität beeinträchtigt. In der vorliegenden Arbeit war die Bodensaugspannung auf der Grünfläche in der obersten Bodenschicht nur leicht erhöht gegenüber der Ackerfläche, so dass die Konkurrenz um Wasser nicht wesentlich höher als auf der Ackerfläche war. Die Bodensaugspannung wurde vor allem von den klimatischen Verhältnissen, weniger von der Bodenvegetation beeinflusst. Die Konkurrenz um Wasser zwischen Gehölzen und der Begleitvegetation hat nach Gakis et al. (2004) vor allem unter trockenen Bedingungen einen Einfluss auf den Zuwachs der gepflanzten Gehölze. In der vorliegenden Arbeit nahm die Bodensaugspannung allerdings keine kritischen Werte an. Unter eher humiden Bedingungen ist es vor allem die Nährstoffkonkurrenz mit der Begleitvegetation, welche den Zuwachs der Gehölze negativ beeinträchtigt. Eine erhöhte Nährstoffverfügbarkeit, welche sich in einer höheren Nährstoffkonzentration in den Blatt-Trockenmasse widerspiegelt, beeinflusst das Gehölzwachstum positiv (Benson et al., 1992). Die P-Konzentration in den Blättern der Pappelstecklinge betrug in der vorliegenden Arbeit auf der Grünlandfläche nur die Hälfte im Vergleich zu den Pappelstecklingen auf der Ackerfläche. Die deutlich geringeren P-Konzentrationen auf der Grünlandfläche, welche sich beim Pappelhybriden "NE 42" nahe dem kritischen Grenzwert (van den Burg, 1990) befand, lassen auf eine stärkere Nährstoffkonkurrenz in der oberen Bodenschicht mit der Begleitvegetation schließen. Bei den bewurzelt gepflanzten Gehölzen war kein Unterschied in der P-Versorgung auf der Acker- und der Grünlandfläche messbar. Van den Driessche (1999) führte eine geringe P-Versorgung von Pappelstecklingen auf eine unzureichende Wurzelentwicklung zurück, welche die Pappeln das Phosphatvorkommen, trotz Düngung, nicht ausschöpfen ließ. Dabei führte eine Düngung der Stecklinge nach der Pflanzung zu erhöhten Zuwächsen, insbesondere wenn die Düngung in unmittelbarer Nähe der Stecklinge und in Abwesenheit von 
Konkurrenzvegetation durchgeführt wurde (van den Driessche, 1999). Neben der erhöhten Konkurrenz um Nährstoffe auf der Grünlandfläche, waren die Nährstoffvorräte auf der Grünlandfläche auch geringer als auf der zuvor landwirtschaftlich genutzten Ackerfläche. Vor allem die Kaliumvorräte waren auf der Ackerfläche wesentlich höher, was zu einer besseren Kalium-Versorgung aller gepflanzten Gehölze führte. Die Kalium-Versorgung korrelierte signifikant positiv mit den Zuwächsen der Stecklinge und den bewurzelten Baumschulpflanzen.

\subsection{Flächenvorbereitung}

Vor der Anlage einer Energieholzplantage ist es notwendig, die Fläche mechanisch oder chemisch zu bearbeiten, um den Konkurrenzdruck der natürlichen Begleitvegetation zu reduzieren und die Anwuchsrate der Stecklinge und jungen Bäume zu erhöhen. Eine intensive Flächenvorbereitung bietet die Möglichkeit auch kleine Pflanzensortimente kostengünstig auszubringen (Pampe et al., 2001). Vor der Pflanzung durchgeführte Maßnahmen können die krautige Konkurrenzvegetation effektiv eindämmen, was sich über die erste Vegetationsperiode hinaus positiv auf die gepflanzten Gehölze auswirken kann (Wittwer et al., 1986). Eine intensive Flächenvorbereitung hatte bei Nilsson und Örlander (1999) sogar nach 18 Vegetationsperioden noch einen positiven Einfluss auf das Bestandesvolumen der Weihrauch-Kiefer (Pinus taeda).

Auf der Versuchsfläche Hamerstorf verringerte eine intensive mechanische Bodenbearbeitung vor der Flächenbegründung den Konkurrenzdruck der Begleitvegetation und erhöhte den Anwuchserfolg und Zuwachs der Gehölze. Durch eine Bodenbearbeitung kann die Konkurrenzvegetation auf Ackerflächen über ein bis zwei Vegetationsperioden gehemmt und der Anwuchserfolg von Erstaufforstungen erheblich verbessert werden (Lüdemann, 1993; Anton, 1999). In einer Studie von Craven et al. (2009) wurden die Effekte der Herbizidanwendung und mechanischen Pflege auf das Wachstum und die Mortalität der Sämlinge von Terminalia amazonia und Tectona grandis auf von Saccharum spontaneum dominierten Grassflächen 
untersucht. Beide Arten erreichten eine größere Höhe, einen dickeren Wurzelhalsdurchmesser und eine geringere Mortalität mit Intensivierung der mechanischen Pflegemaßnahmen und Herbizidanwendung. Höhe und Konkurrenz von S. spontaneum korrelierte negativ mit der Intensität der mechanischen und chemischen Begleitwuchsregulierung. In der vorliegenden Studie war die mechanische Bodenbearbeitung effektiver bei der Eindämmung der Begleitvegetation als der Herbizideinsatz, was sich in signifikant höheren Anwuchs- und Zuwachsraten widerspiegelte. Auch bei Geyer (2009) war die Herbizidanwendung zur Begleitwuchsregulierung weniger effektiv als angenommen, was allerdings durch eine zu späte Ausbringung der Herbizide im Frühjahr erklärt wurde; dadurch erhielten die Herbizide zu wenig Feuchtigkeit zum Aktivieren und die stark aufkommende Konkurrenzvegetation reduzierte den Höhenzuwachs der jungen Pappeln.

Besonders auf Grünlandflächen ist der Umbruchaufwand für eine Steckholzpflanzung unumgänglich (Hofmann, 2004). Bei lediglich gefrästen Grünlandteilen wird die erforderliche Bodenlockerung und Zerschlagung des Graswurzelfilzes für die Steckung zwar zunächst erreicht; durch unmittelbar folgendes Weiterwachsen der Graswurzeln werden die Steckhölzer jedoch aufgrund starker Konkurrenz um Wasser und Licht beeinträchtigt. Auf der Grünlandfläche in Gütersloh wurde versucht, den Herbizideintrag und den Nitrataustrag nach der Bodenbearbeitung möglichst gering zu halten, um die Belastung des Sickerwassers so gering wie möglich zu halten. Deshalb wurden die Pflanzreihen auf der Grünlandfläche streifenweise bearbeitet. Im November vor der Pflanzung wurden die Pflanzreihen chemisch behandelt und vor der Pflanzung erfolgte eine Bodenauflockerung, um die Pflanz- und Steckarbeiten zu erleichtern. Da die Pflanzreihen schnell wieder von den umliegenden Gräsern bewachsen wurden und sich die Pflugspuren für Mäuse zudem als idealer Zugangsweg zu den Pflanzenwurzeln erwiesen, kann diese Form der Flächenvorbereitung unter den gegebenen Umständen nicht empfohlen werden. Dieselben Baumarten erbrachten auf der flächig gepflügten Ackerfläche höhere Anwuchs- und Zuwachsraten als auf der streifenweise vorbereiteten Grünlandfläche. Das flächige Pflügen einer Grünfläche resultierte bei Otsamo et al. (1995) in dreifach 
höheren Volumenzuwächsen von Acacia mangium nach sechs Jahren im Vergleich zum streifenweisen Pflügen, was durch eine reduzierte Graskonkurrenz auf den flächig gepflügten Flächen erklärt wurde; Deckungsgrad und Höhe des auf der Fläche dominierenden Japanischen Blutgrases (Imperata cylindrica) waren auf den flächig gepflügten Flächen geringer als auf den streifenweise gepflügten Flächen. Das Gras hat die gepflügten Streifen von den nicht gepflügten Streifen aus schnell wieder bewachsen können, was zu einer erhöhten Konkurrenz geführt und das Wachstum der Bäume gehemmt hat (Otsamo et al., 1995). Wenn aus Gründen des Trinkwasserschutzes auf eine Bodenbearbeitung verzichtet wird, muss damit gerechnet werden, dass sich die Kultur spürbar verteuert oder die Qualität des Jungbestandes sinkt (Pampe et al., 2001).

Ein Nachteil einer mechanischen Bodenbearbeitung ist ein erhöhter Nitrataustrag mit dem Sickerwasser. Bei der landwirtschaftlichen Nutzung von Flächen wird durch Stickstoff-Düngemittel und Bodenbearbeitung jedes Jahr eine erhebliche Menge Nitrat mit dem Sickerwasser ausgetragen, was zu erheblichen Problemen bei der Grundwasserqualität in Trinkwassereinzugsgebieten führen kann. Auf sandigen landwirtschaftlich genutzten Böden können die Nitratausträge das 2- bis mehr als 4fache der erlaubten Nitratkonzentration von $50 \mathrm{mg} / \mathrm{l}$ betragen (Strebel et al., 1989). Auch eine Veränderung der Bewirtschaftung, wie der Umbruch von Grünland, führt zu erheblichen Nitratausträgen. Durch die einsetzende Mineralisation nach dem Umbruch erfolgt ein Anstieg der Nitratauswaschung (Strebel et al., 1989). In der vorliegenden Arbeit stieg der Nitrataustrag nach dem Pflügen der Fläche schlagartig an, sank innerhalb eines halben Jahres unter den erlaubten Grenzwert ab und blieb durch eine dauerhafte Bodenruhe konstant niedrig. Landwirtschaftliche Flächen nicht zu pflügen, resultierte auch bei Schindler und Müller (2009) in einem reduzierten Nitrataustrag (18 kg/ha) im Vergleich zur Pflugvariante (27 kg/ha). Untersuchungen zu Nitratausträgen nach Ackeraufforstungen zeigten, dass die Nitrat-Konzentrationen im Sickerwasser der unbearbeiteten Kontrolle während der ersten Vegetationsperiode signifikant niedriger waren als auf allen bearbeiteten Varianten (Pampe et al., 2001). Der Nitrataustrag kann unter Energieholzplantagen durch die langfristige Bodenruhe 
sowie das Aussetzen des jährlichen Düngereinsatzes wie in der Landwirtschaft daverhaft reduziert werden. Nitrat-Auswaschungsraten sind unter Waldflächen grundsätzlich niedriger als unter landwirtschaftlich intensiv genutzten Flächen (Strebel et al., 1989 ; Evers, 2001$).$

\subsection{Begleitwuchsregulierung}

\subsubsection{Mähen}

Durch das Mähen zwischen den Pflanzreihen wurde nicht nur die oberirdische Biomasse der Begleitvegetation deutlich reduziert, sondern auch die Artenzusammensetzung beeinflusst. Die ansonsten von einer Mischung aus unterschiedlichen Gräsern zusammengesetzte Grünflächenvegetation wurde nach dem Mähvorgang deutlich vom Deutschen Weidelgras (Lolium perenne) dominiert. Ähnliches wurde von Willoughby und McDonald (1999) beobachtet, als auf einer gemähten Fläche der Anteil von Weiß-Klee (Trifolium repens) und dem Deutschen Weidelgras deutlich anstieg, da diese sich vegetativ vermehrenden Arten tolerant gegenüber dem Mähvorgang sind. Vergleichbare Entwicklungen zeigten sich auf der Ackerfläche, auf der infolge des Mähens der Windenknöterich (Fallopia convolvulus) und das Acker-Stiefmütterchen (Viola arvensis) vermehrt aufkamen. Diese erreichen nur eine geringe Wuchshöhe und bleiben somit vom Mäher verschont.

Der Einfluss der Pflegemaßnahmen auf das Anwuchs- und Zuwachsverhalten der verschiedenen Baumarten war sehr unterschiedlich. Baumarten reagieren unterschiedlich auf Stress, wodurch ihre Fähigkeit, mit der natürlichen Vegetation zu konkurrieren, beeinflusst wird (Kolb et al., 1990). Bei Vandenberghe et al. (2006) wurde die Überlebens- und Zuwachsrate junger Buchen (Fagus sylvatica), Fichten (Picea abies) und Bergahorne (Acer pseudoplatanus) positiv durch das Abmähen der Begleitvegetation beeinflusst. Ähnlich positive Effekte der mechanischen Begleitwuchsregulierung wurden auch in anderen Untersuchungen gemessen 
(Czapowskyi \& Safford, 1993; Coll et al., 2004; Vandenberghe et al., 2008; Craven et al., 2009). In anderen Studien wurde durch das Mähen ein negativer bzw. gar kein Effekt bezüglich Überlebens- und Zuwachsraten von Gehölzen beobachtet (Löf \& Welander, 2004; Coll et al., 2007; Skousen et al., 2009).

In der vorliegenden Arbeit beeinflusste die mechanische Begleitwuchsregulierung die die Überlebens- und Zuwachsraten Baumarten spezifisch. Die mechanische Begleitwuchsregulierung hatte dabei keinen signifikant positiven Effekt auf das Anwuchsverhalten im ersten Jahr und auch nicht auf die Überlebensrate nach zwei Vegetationsperioden. Das Mähen der Fläche führte zu höheren Zuwächsen der Weidenstecklinge und Aspen in der ersten Vegetationsperiode im Vergleich zur Kontrollfläche, welche allerdings nach dem zweiten Standjahr nicht mehr messbar waren. Nach der zweiten Vegetationsperiode waren der Bergahorn auf der Ackerfläche und die Robinie auf der Grünlandfläche signifikant höher, wenn zwischen den Reihen gemäht wurde. Außerdem wurde im ersten Jahr eine positive Tendenz des Mähens auf die Anwuchsrate der Pappelstecklinge festgestellt. Ohne die konkurrierende Begleitvegetation, insbesondere der Gräser, bilden die Stecklinge mehr Feinwurzeln aus (Coll et al., 2007). Auf Versuchsflächen in Maine war die Überlebensrate von Pappelhybriden ebenso wie die produzierte Biomasse auf nicht gemähten Flächen gegenüber gemähten Flächen über 10 Jahre hinweg deutlich reduziert (Czapowskyi \& Safford, 1993). In der vorliegenden Studie war der Unterschied der Überlebensrate der Pappelstecklinge auf der gemähten Fläche zur Kontrollfläche nach der zweiten Vegetationsperiode kaum noch vorhanden. Das Nachlassen dieses positiven Effektes in der zweiten Vegetationsperiode könnte durch vermehrte Aufkommen einkeimblättriger Kräuter, insbesondere des Deutschen Weidelgrases (Lolium perenne) im zweiten Jahr erklärt werden. So zeigte sich in der ersten Vegetationsperiode eine Abhängigkeit der Anwuchsrate der Pappelstecklinge von der Biomasse der einkeimblättrigen Kräuter und des Deutschen Weidelgrases. Dabei hatte die Vornutzung im ersten Jahr einen wesentlich größeren Einfluss als die Begleitwuchsregulierung auf den Anteil einkeimblättriger Kräuter und damit auf die Überlebensraten. Denn auf der Grünlandfläche war der Anteil vor allem der 
schädlichen Gräser wie des Deutschen Weidelgrases trotz Begleitwuchsregulierung wesentlich höher als auf der Ackerfläche ohne Pflegemaßnahmen. Auch bei Coll et al. (2007) hatte keine der im ersten Jahr der Pflanzung durchgeführte Begleitwuchsregulierung einen Einfluss auf die oberirdische Wuchsentwicklung von Pappelhybriden; und auch bei den zweijährigen Pappeln wurden keine positiven Effekte einer mechanischen Begleitwuchsregulierung festgestellt. Einen positiven Einfluss hatte das Mähen der Ackerfläche auf die Überlebensrate der Aspe und Robinie nach zwei Vegetationsperioden, deren Überlebensraten mit dem Deckungsgrad der natürlichen Konkurrenzvegetation negativ korrelierten. Die Reduktion des Deckungsgrades der natürlichen Bodenvegetation führte zu höheren Überlebensraten der Aspe und Robinie.

Eine signifikant geringere Anwuchsrate infolge des Mähens wurde auf der Ackerfläche bei Bergahorn und Schwarzerle und auf der Grünlandfläche bei Robinie ermittelt. Das Mähen hatte bei Bergahorn und Schwarzerle auch nach der zweiten Vegetationsperiode noch einen signifikant negativen Effekt auf die Überlebensrate auf der Ackerfläche. Bei der Schwarzerle war ebenfalls der Höhenzuwachs signifikant geringer wenn die Ackerfläche gemäht wurde. Die Überlebensrate des Weidenstecklings war nach dem zweiten Standjahr ebenfalls auf Acker- und Grünlandfläche signifikant geringer als bei der Kontrollvariante. Auch bei Skousen et al. (2009) war die Überlebensrate einjähriger Gehölze auf ungemähten Flächen am höchsten. Nach 7 Jahren hatte das Mähen der Fläche das Überleben der Spätblühenden Traubenkirsche (Prunus serotina), Roteiche (Quercus rubra) und des Tulpenbaumes (Liriodendron tulipifera) reduziert, aber nicht das der Echten Walnuss (Juglans nigra) und Weiß-Esche (Fraxinus americana) (Skousen et al., 2009 war die Überlebensrate einjähriger Gehölze auf ungemähten Flächen am höchsten. Nach 7 Jahren hatte das Mähen der Fläche das Überleben der Spätblühenden Traubenkirsche (Prunus serotina), Roteiche (Quercus rubra) und des Tulpenbaumes (Liriodendron tulipifera) reduziert, aber nicht das der Echten Walnuss (Juglans nigra) und WeißEsche (Fraxinus americana) (Skousen, 2009). Nach Löf und Welander (2004) hat das alleinige Mähen keinen Effekt auf das Wachstum von Eichen und Buchen. Denn für 
eine effektive Unkrautregulierung reicht nicht aus, die Begleitvegetation nur oberflächlich zu entfernen, sondern es muss zusätzlich die Konkurrenz zwischen den Wurzeln beseitigt werden (Davis et al., 1999). So zeigten auch Sämlinge der schattentoleranten Buche einen höheren Durchmesser- und Höhenzuwachs ohne Beschattung und Wurzelkonkurrenz (Coll et al., 2004). Vor allem Gräser bedeuten eine hohe Wasser- und Nährstoffkonkurrenz und führen zu einer limitierten Photosyntheserate und Wurzelabsorption, wodurch das Wachstum der jungen Bäume reduziert wird. In der vorliegenden Arbeit könnte der negative Effekt des Mähens auf die Überlebensrate, insbesondere bei der Schwarzerle, auf eine verstärkte Austrocknung des Bodens ohne die schützende Vegetationsdecke hervorgerufen worden sein. Hierdurch wurden die ohnehin unter Trockenstress leidenden Gehölze noch stärker gestresst, was zu einer höheren Ausfallrate führte. Die Bodensaugspannungen waren auf den gemähten Flächen höher als auf den Kontrollflächen mit Vegetationsdecke. Die durch Trockenheit verursachte Ausfallrate war auf der gemähten Teilfläche doppelt so hoch wie auf der Kontrollfläche und der Variante mit eingesäten Nutzpflanzen.

\subsubsection{Nutzpflanzen}

Die Einsaat von Nutzpflanzen führte insbesondere in der ersten Vegetationsperiode zu einer deutlichen Reduktion der von Gräsern und Kräutern gebildeten Trockenbiomasse, wobei eine flächendeckende Bodenbearbeitung und Nutzpflanzeneinsaat auf der Grünfläche das Unkraut wahrscheinlich effektiver hätte verdrängen können. Die infolge des Herbizideinsatzes vegetationsfreien Pflanzstreifen wurden von den umliegenden Gräsern rasch wieder bewachsen, bevor die Nutzpflanzen vollständig auflaufen konnten. Dass Nutzpflanzendecken nicht in der Lage sind, eine vorhandene wuchskrättige Vegetation zurückzudrängen (Reinecke, 1990), könnte das völlige Ausbleiben der Kleesaat auf der Grünfläche erklären. Auf der bei Kulturbegründung vegetationsfreien Ackerfläche zeigte sich ohne den Konkurrenzdruck der Unkräuter ein deutlich höheres Aufkommen der Nutzpflanzen als auf der Grünlandfläche. Die 
Entwicklung der Klee-Einsaat wurde durch die flächige Bodenbearbeitung deutlich gefördert.

Die Nutzpflanzeneinsaat wirkte sich sehr unterschiedlich auf die Überlebensrate der verschiedenen Gehölzarten aus. Zumeist war der Effekt der eingesäten Nutzpflanzen nicht signifikant. Ausnahmen waren die Überlebensraten der Aspe auf Acker und der Weidenstecklinge auf Acker und Grünland, deren Überlebensraten mit Nutzpflanzeneinsaat signifikant geringer als bei den Kontrollpflanzen ausfielen. Die Überlebensrate der Robinie war mit Nutzpflanzeneinsaat signifikant höher als ohne. Auch bei Evers (2001) beeinflussten die Hilfspflanzendecken die Entwicklung der gepflanzten Bäume relativ gering und nicht einheitlich. Nach Boelcke (2006) können Untersaaten zur Unkrautunterdrückung und Bodenbedeckung nicht empfohlen werden, da sie für Baumsprösslinge eine starke Konkurrenz darstellen. Auch Jylhä et al. (2006) räumten die Möglichkeit ein, dass sich die Konkurrenz um Ressourcen zwischen eingesätem Weißklee und Baumsämlingen schwerwiegender auf die Ausfallhäufigkeit auswirkt als die Konkurrenz mit der natürlichen Vegetation. In diesem Sinne führte der von Wolf und Böhnisch (2003) eingesäte Weißklee zwar zu einer Verdämmung der Konkurrenzflora, aber auch zur Verdämmung der in der Anwuchsphase besonders auf Licht angewiesenen Stecklinge. Dies äußerte sich in etwa vierfach höheren Ausfallraten des Pappelklones "Max 4" im Vergleich zur Kontrollfläche, wo die Ausfallrate bei etwa $10 \%$ lag. Ähnliches wurde in der vorliegenden Arbeit bei den verwendeten Pappelund Weidenstecklingen beobachtet. Die Nutzpflanzen reduzierten die Überlebensraten der gepflanzten Stecklinge. Vor allem bei den Weidenstecklingen waren diese Effekte signifikant. Untersuchungen zu Erstaufforstungen von Evers (2001) ergaben keine Unterschiede in den Ausfallhäufigkeiten von Buchen in Abhängigkeit von Roggen (Secale cereale) oder Senf (Sinapis alba) und Ölrettich (Raphanus sativus) als Hilfspflanzen. Die Mortalität der Bergahorne war in den Varianten mit Senf/Ölrettich höher als mit Roggeneinsaat und ohne Hilfspflanzen. Bei Evers (2001) führte die Einsaat von Hilfspflanzen (Roggen; Senf/Ölrettich) zwar zu geringeren Deckungsgraden der Gräser, aber mit Senf/Ölrettich-Einsaat übten Ölrettich und Gräser eine ebenfalls hohen Konkurrenzdruck auf den Bergahorn aus. 
Die Einsaat der Nutzpflanzen führte im Vergleich zu den Kontrollflächen zu nicht signifikanten oder signifikant negativen Wuchsleistungen der unterschiedlichen Gehölzarten. Die geringeren Zuwächse vor allem auf der Ackerfläche können ebenso wie die teilweise reduzierten Anwuchsraten durch eine erhöhte interspezifische Konkurrenz mit der üppig aufgelaufenen Nutzpflanzendecke erklärt werden. Die von den eingesäten Nutzpflanzen und der natürlichen Begleitvegetation gebildete Trockenbiomasse war auf der Ackerfläche höher als die Biomasse der natürlichen Vegetation auf den Kontrollflächen. Untersuchungen von Jantzen (1989) verdeutlichten die Konkurrenzkraft einer Leguminosenmischung mit der Hauptbaumart, wobei die besten Wuchsdaten bei der geringsten Vegetationsdecke, die sich nur aus Standortsvegetation zusammensetzte, gemessen werden konnten. Auch bei Gakis et al. (2004) zeigte der eingesäte Weißklee einen negativen Effekt auf Acer pseudoplatanus und Pinus sylvestris, deren Zuwächse mit eingesätem Weißklee signifikant geringer waren als auf den Kontrollflächen. Die Untersuchungen zur Nährstoffversorgung der Pflanzen zeigten signifikant niedrigere P-Konzentrationen der Gehölze mit Nutzpflanzeneinsaat, was auf eine gesteigerte intraspezifische Konkurrenz um diesen Nährstoff hindeutet. Die Konkurrenz um Wasser wurde durch die auf der Ackerfläche üppig aufgelaufene Nutzpflanzendecke ebenfalls erhöht, was sich in höheren Bodensaugspannungen gegenüber der Kontrollfläche äußerte. Untersuchungen zur Bodenfeuchte und Nährstoffversorgung von Davies (1985) verdeutlichten, dass die Konkurrenz mit der Begleitvegetation um Wasser und Nährstoffe das Überleben und den Zuwachs von jungen Bäumen reduziert.

\subsection{Bodenhilfsstoffe}

\subsubsection{Mykorrhiza}

Beziehungen zwischen Mikroorganismen und Pflanzen sind bestimmende Faktoren für die Pflanzenvitalität. Das Vorkommen von Bodenmikroorganismen wird aber durch 
eine langfristige landwirtschaftliche Nutzung der Böden vermindert und damit auch ihre günstigen Eigenschaften für Gehölze (Jeffries, 2001). Arbuskuläre Mykorrhizen sind dabei die wichtigsten Symbiosepartner von Gehölzen, welche unter P-Limitierung die Pflanzenentwicklung, die Nährstoffaufnahme, Wasserverfügbarkeit und Biomasseproduktion beeinflussen können (Jeffries et al., 2003). Mykorrhizen dienen ebenfalls als Schutz gegen Pathogene und schädliche Umwelteinflüsse (Jeffries et al., 2003). Es findet sich eine beachtliche Menge an Literatur, die sich mit der Wirkung von Ektomykorrhizen und vesikulär-arbuskulären Mykorrhizen (VAM) auf das Überleben, den Zuwachs und die Vitalität von landwirtschaftlichen und forstlich relevanten Pflanzen beschäftigen (Marx et al., 2002). Laut Fitter und Garbaye (1994) ist es allerdings schwierig, in Feldversuchen die Effekte von Mykorrhizen von denen anderer Mikroorganismen zu separieren. Ein Problem bei Feldversuchen ist ebenso der starke klimatische Einfluss wie Sommertrockenheit, welche die Ausbildung von Mykorrhizen reduzieren kann (Feil et al., 1988; Nilsen et al., 1998).

In der vorliegenden Arbeit fiel der Einfluss der Mykorrhizierung mit dem VAMykorrhizapilz Glomus intraradices auf den Anwuchs, die Vitalität und den Höhenzuwachs der Gehölze sehr unterschiedlich aus. Die Anwuchs- und Überlebensraten der Gehölze auf der Versuchsfläche Sudheide (Gütersloh) wurden allenfalls signifikant negativ von der Mykorrhizierung beeinflusst, besonders bei der Eberesche und Schwarzerle waren die Anwuchszahlen der mykorrhizierten Pflanzen gegenüber den Kontrollpflanzen deutlich geringer. Bei den anderen Baumarten unterschieden sich die Anwuchsraten der mykorrhizierten und nicht-mykorrhizierten Pflanzen nicht. Auf der Versuchsfläche Unterlüß (Uelzen) war ebenfalls kein signifikanter Einfluss der Mykorrhizierung auf die Anwuchsrate der drei Pappelstecklinge festzustellen. Auch bei Marx et al. (2002) hatten junge mit Pisolithus tinctorius beimpfte Kiefern auf Aufforstungsflächen ebenso hohe Überlebens- und Zuwachsraten wie die Kiefern, die mit natürlich vorkommenden TelephoraEktomykorrhizen besiedelt waren. Bei Evers und Pampe (2005) wurden junge Pflanzen der Buche (Fagus sylvatica) und des Bergahorns (Acer pseudoplatanus) auf ehemals 
landwirtschaftlichen Flächen in Deutschland gepflanzł und mit Paxillus involutus mykorrhiziert. Die Überlebensrate der mykorrhizierten Buchen war signifikant höher als die der Kontrollpflanzen. Beim Bergahorn wurden keine signifikanten Unterschiede in der Überlebensrate festgestellt.

Die Vitalität der Gehölze wurde kaum von der Mykorrhizierung beeinflusst. Die Mykorrhizierung hatte aber einen signifikant positiven Einfluss auf die Höhenzuwächse des Bergahorns, der Eberesche und des Pappelstecklings "Max 4" auf der Versuchsfläche Sudheide (Gütersloh). Auch bei Evers und Pampe (2005) hatte eine Mykorrhizierung einen positiven Effekt auf den Höhenzuwachs des Bergahorns. Die Höhe des Bergahorns nach vier Jahren durchschnittlich $30 \mathrm{~cm}$ höher als die der unbehandelten Kontrollen; der Wurzelhalsdurchmesser war ebenfalls signifikant höher, während bei der Blattanalyse keine Veränderungen der Nährstoffversorgung festgestellt wurden. Ein Hauptvorteil der Mykorrhizierung liegt auf nährstoffreichen Böden nach Evers und Pampe (2005) in einer höheren Trockenresistenz der Pflanzen. Berman und Bledsoe (1998) stellten fest, dass mit einer zunehmenden Mykorrhizierung der Höhenzuwachs auf Kosten des Wurzelwachstums stieg. Die MykorrhizierungsVersuche von Munro et al. (1999) resultierten in größeren und schwereren Pflanzen und einer höheren Zuwachsrate der mykorrhizierten Pflanzen im Vergleich zu den Kontrollvarianten. Die Ergebnisse von Baum et al. (2002) zeigten auf, dass eine Mykorrhizierung die Wachstumsrate von Balsampappeln (Populus trichocarpa) auf ehemals landwirtschaftlich genutzten Flächen in der ersten Vegetationsperiode steigern kann. Dadurch können die Widerstandskraft gegen Pathogene und die Konkurrenzkraft um Nährstoffe gegenüber der natürlichen Bodenvegetation gestärkt werden. Ein gesteigertes Stecklingswachstums von Pappeln im ersten Jahr nach der Pflanzung bedeutet einen erheblichen Vorteil gegenüber der Konkurrenzvegetation, welche die Stecklinge leicht ausdunkeln kann (Baum et al., 2002). Zusätzlich reduziert ein erhöhtes Wachstum der Stecklinge die Gefahr gegen Mäusefraß (Bellocq \& Smith, 1995). 
Beim Pappelsteckling "Max 4" wurde eine verbesserte Nährstoffversorgung der mykorrhizierten Pflanzen im Vergleich mit den Kontrollpflanzen festgestellt, welche mit einem höheren Zuwachs der Stecklinge korrelierte. Die verbesserte NährstoffVersorgung der mykorrhizierten Pappelstecklinge unterstützł die These von van den Driessche (1999), dass das Fehlen von passenden Mykorrhiza-Pilzen zu einer unzureichenden P-Versorgung von Pappelstecklingen in seinen Düngungsversuchen geführt haben könnte. Bei Heslin und Douglas (1985) steigerten Ektomykorrhizapilze die Nährstoffversorgung und Wurzelbildung von Pappelhybriden. Bei Baum et al. (2000) hat ein Beimpfen mit Mykorrhizen allerdings zu einer reduzierten N- und PKonzentration bei Pappeln geführt, was durch eine gesteigerte Stammbiomasse erklärt wurde. Auch bei Allen et al. (2003) hat das Animpfen mit Mykorrhizapilzen die Biomassebildung, aber nicht die P-Konzentration, der Gehölze gesteigert, auch wenn zahlreiche Studien ein Zusammenhang zwischen erhöhter Biomasse und PKonzentration aufgezeigt haben (Smith \& Read, 1997).

Mit Ausnahme der Eberesche, dem Bergahorn und des Pappelhybriden "Max 4" reagierten die anderen Baumarten auf der Versuchsfläche Sudheide (Gütersloh) nicht signifikant auf die Mykorrhizierung. Und auch auf der Versuchsfläche Unterlüß (Uelzen) war zwischen mykorrhizierten und nicht mykorrhizierten Pappelstecklingen kein Unterschied messbar. In der Literatur finden sich ebenfalls Beispiele für eine wirkungslose Mykorrhizierung von Gehölzen. Bei Oliveira et al. (2005) hatte Glomus intraradices keinen positiven Effekt auf Höhe, Wurzelhalsdurchmesser und Biomasse der Schwarzerle. Die Mykorrhizierung mit Glomus intraradices hatte ebenfalls keinen signifikanten Einfluss auf das Wachstum von Acer platanoides, Sorbus aucuparia und zwei weiteren von Morrison et al. (1993) mykorrhizierten Baumarten. Eine Mykorrhizierung von Robinia pseudoacacia erbrachte auch bei Ferrari und Wall (2008) keinen verbesserten Zuwachs der Pflanzen.

Auf der Grünlandfläche in Gütersloh war der Einfluss der Mykorrhizierung auf den Höhenzuwachs durchweg negativ, während er auf der Ackerfläche zumeisł positiv, wenn auch nicht signifikant positiv, war. Ein Beimpfen mit Mykorrhiza-Pilzen kann zu 
einem verringerten Pflanzenwachstum führen (Haselwandter \& Bowen, 1996). Bei Luo et al. (2009) hatten Pappeln (Populus euphratica), die mit Paxillus involutus mykorrhiziert wurden $27 \%$ weniger Wurzel- und $21 \%$ weniger Stammbiomasse als die nicht mykorrhizierten Pflanzen. Eine erhöhte Bodenfruchtbarkeit, insbesondere was den P- und N-Gehalt angeht, kann die Ausbildung von Mykorrhizen und/oder der Mykorrhiza-Vielfalt fördern, aber die Wirkung ist zumeist abhängig von der Pflanzenund Pilzsorte (Dell, 2002). Der P-Gehalt im Bodensubstrat korreliert oft mit der prozentualen Anzahl von mykorrhizierten Wurzeln (Maldonado et al., 2000). Baum et al. (2000) stellten fest, dass auf nährstoffreichen Böden die Stammlänge, Stammmasse und das Stamm-Wurzel-Verhältnis von Pappelklonen durch Ektomykorrhizen erhöht wurden, während auf Böden mit einem geringen $\mathrm{C}_{\text {org }}$ und $\mathrm{N}$ Gehalt nur das Verhältnis von Stamm zu Wurzel erhöht wurde. Die Wurzelbiomasse und die absolute N-Akkumulation in der Biomasse von Trieb und Wurzel wurden nur von dem Pflanzsubstrat, nicht von der Mykorrhizierung, beeinflusst. Dosskey (1990) nennt als Grund für ein verringertes Wachstum nach der Mykorrhizierung, dass applizierte konkurrenzkräftige Pilze die wirkungsvolleren Pilze verdrängen, die natürlich auf der Fläche vorkommen; ein anderer Grund sei, dass ein sehr gut entwickeltes Pilzwachstum das Pflanzenwachstum durch das starke externe Wachstum der Pilze reduzieren kann. Die Nachteile des externen Wachstums müssen somit gegen die Vorteile einer verbesserten Nährstoffaufnahme auf armen Böden abgewogen werden, wobei ein verstärktes Wachstum der Mykorrhiza-Pilze vor allem auf nährstoffreicheren Böden vorkommt, wo die positive Effekte einer verbesserten Nährstoffaufnahme keine so große Rolle spielen (Haselwandter \& Bowen, 1996).

\subsubsection{Hydrogel}

Vorhergehende Untersuchungen über die Wirkung von Hydrogel verfolgten zwei Schwerpunkte. Ein Aspekt betraf eine erhöhte Überlebensrate von Aufforstungen in ariden und semiariden Gebieten. Der andere Aspekt betraf eine erhöhte Produktivität 
im landwirtschaftlichen Bereich. Viero und Little (Viero \& Little, 2006) haben den Effekt von verschiedenen Pflanzmethoden, Hydrogel eingeschlossen, auf die Überlebens- und Zuwachsrate von Eukalyptus in Südafrika untersucht. Die Hydrogelbeigabe konnte sowohl die Anzahl der angewachsenen Pflanzen als auch die Zuwächse unter suboptimalen Pflanzbedingungen erhöhen. Bei Savé et al. (1995) konnten junge Pinien (Pinus pinea), mithilfe von Hydrogel 1,4-2 mal längere Trockenperioden überleben als die Kontrollpflanzen ohne appliziertes Hydrogel. Auch in den Untersuchungen von Hüttermann et al. (1999) hatten junge Aleppo-Kiefern (Pinus halepensis) höhere Überlebensraten, wenn dem Bodensubstrat Hydrogel beigemischt wurde. Die Wasserspeicherfähigkeit des sandigen Bodensubstrates stieg exponentiell mit zunehmender Hydrogel-Konzentration und die Überlebensrate der Aleppo-Kiefern war mit einer 0,4\%igen Hydrogel-Konzentration doppelt so hoch wie die Überlebensrate der Kontrollpflanzen. Ähnliche Ergebnisse wurden von Al-Humaid und Moftah (2007) mit der Knopfmangrove (Conocarpus erectus) erzielt. Wenn dem Bodensubstrat Hydrogel beigemischt wurde überlebten die jungen Mangroven unter Trockenstress dreimal länger als die Kontrollpflanzen, und auch das Trieb- und Wurzelwachstum wurde durch die Hydrogel-Beigabe signifikant erhöht. Die Forschungsergebnisse von Al-Humaid und Moftah (2007) bestätigten die Annahme, dass eine Anreicherung des Bodensubstrates mit 0,4-0,6\% Hydrogel die Trockentoleranz von Anpflanzungen in ariden und semiariden Gebieten erhöhen kann. Hydrogel kann den pflanzenverfügbaren Wassergehalt im Boden um das 2-3 fache erhöhen, wodurch die Bewässerung von Anpflanzungen, welche in ariden und semiariden Gebieten ein großes Problem darstellt, signifikant reduziert werden kann (Koupai et al., 2008). Untersuchungen von Rowe et al. (2005) ließen vermuten, dass Hydrogel ebenfalls den Nährstoffaustrag reduzieren kann, aber auch einen negativen Effekt auf die $\mathrm{N}$ Verfügbarkeit hat.

Forschungsergebnisse aus Bosnien zeigten, wie wichtig die applizierte HydrogelKonzentration für eine erfolgreiche Aufforstung ist, da eine falsche Anwendung zu zwei Problemen führen kann (Visnjic, 2006). Im Fall von hohen Niederschlagsmengen kann eine zu hohe Hydrogel-Konzentration zu anaeroben Bedingungen im Boden 
führen und die jungen Gehölze ersticken (Woodhouse \& Johnson, 1991). In den Untersuchungen von Sarvas et al. (2007) über die Anwendung von Hydrogel, wurden in der ersten Vegetationsperiode die geringsten Überlebensraten (64\%) von jungen Kiefern gemessen, wenn bei der Pflanzung Hydrogel appliziert wurde. Diese hohe Sterblichkeit wurde durch eine zu hohe Hydrogel-Dosierung von $7 \mathrm{~g} /$ Pflanzloch erklärt. Die Anwendung von Hydrogel ist einfach, aber es ist wegen der hohen Wasseraufnahme- und Schwellfähigkeit kompliziert, die richtige Dosierung zu finden, ohne eine zu hohe Konzentration zu verwenden. In Bosnien verdeutlichten die Untersuchungsergebnisse von Visniic (2006), dass eine Hydrogelmenge von $10 \mathrm{~g} / \mathrm{dm}^{3}$ Bodensubstrat zu hoch ist, während $5 \mathrm{~g} / \mathrm{dm}^{3}$ signifikant bessere Vitalitätsergebnisse der Gehölze erbrachte als die der Kontrollpflanzen. Da es sich in der vorliegenden Arbeit um einen moderateren Standort in Bezug auf Klima- und Bodenverhältnisse handelt, wurde eine Hydrogel-Konzentration von $2 \mathrm{~g} / \mathrm{dm}^{3}$ Bodensubstrat gewählt, um eine Überdosierung und ein Ersticken der Pflanzen zu vermeiden.

Ein weiterer negativer Effekt, der bei der Anwendung von Hydrogel auftreten kann, ist, dass Hydrogel bei einer zu geringen Bodenfeuchtigkeit das wenige verfügbare Wasser aus dem Boden extrahieren kann, wodurch der Boden gänzlich austrocknet. Hüttermann et al. (1999) stellten fest, dass eine signifikante Menge an dem von Hydrogel absorbierten Wasser den Pflanzen dann nicht mehr zur Verfügung steht. Im Fall von nur geringen Niederschlägen absorbierte Hydrogel das Wasser und verhinderte damit die Wasseraufnahme durch die Pflanze. In der vorliegenden Arbeit wurde die Anwuchsrate der Schwarzerle signifikant erhöht, ansonsten hatte Hydrogel einen negativen Effekt auf das Anwuchsverhalten der gepflanzten Gehölze. Grund des nicht verbesserten Anwuchses der meisten Baumarten könnte der kaum vorhandene Niederschlag nach der Pflanzung (ca. 1 Monat) sein. Das Hydrogel hat die nur geringen Niederschläge nach der Pflanzung absorbiert, und damit die Wasseraufnahme durch die Gehölze verhinderte. Andererseits hatte die HydrogelBeimischung auf die Anwuchsraten einiger Pflanzen gar keine Wirkung. Nach Sarvas et al. (2007) kann Hydrogel ohne zur Verfügung stehendes Wasser zur Absorption, auch keinen Effekt auf die Überlebensrate haben. Auf welche Weise Hydrogel auf 
Pflanzen wirkt, hängt von der Baumart und der angewendeten Hydrogel-Konzentration ab (Tsakaldimi, 1998; Lin et al., 2004; Visnjic, 2006; Sarvas et al., 2007).

Der positive Effekt des Hydrogels auf die Anwuchsrate der Schwarzerle könnte in der höheren Wasserbedürftigkeit der Schwarzerle im Vergleich zu den anderen Baumarten begründet sein. Specht und Harvey-Jones (2000) haben in ihren Untersuchungen festgestellt, dass Baumarten mit einer geringeren Trockentoleranz verstärkł Biomasse akkumuliert haben wenn Hydrogel appliziert wurde, während Baumarten mit einer höheren Toleranz gegenüber Trockenheit keine Verbesserung der Biomassebildung zeigten. In Gebieten, die nicht unter extremer Sommertrockenheit leiden, konnte Hydrogel die Überlebensraten der Gehölze nicht erhöhen, so dass Hydrogel für Aufforstungen mit angepassten Baumarten nicht nötig ist. Die gegenüber Trockenheit empfindlichen Euphrat-Pappel (Populus euphratica) zeigte ebenfalls eine positive Reaktion auf die Beimischung von Hydrogel zum Pflanzsubstrat (Luo et al., 2009). Die mit Hydrogel gepflanzten Pappeln hatten signifikant höhere Stammhöhen (17\%), Wurzelbiomasse (17\%), Stamm- (33\%) und Blattbiomasse (31 \%). Visnjic (2006) konnte keine Unterschiede in der Reaktion von trockentoleranten und nicht toleranten Baumarten auf eine Hydrogel-Zugabe feststellen. In der vorliegenden Arbeit hatte Hydrogel einen signifikant positiven Einfluss auf den Zuwachs der Robinie. Ansonsten wurden keine signifikanten Veränderungen der Zuwächse infolge der HydrogelBeigabe gemessen. Der Hydrogel-Zusatz zeigte aber positive Effekte auf die Vitalität einiger Gehölze. In der ersten oder auch erst zweiten Vegetationsperiode zeigte sich eine verbesserte durchschnittliche Vitalität der noch lebenden Pflanzen einiger Baumarten.

Außer bei der Schwarzerle lässt sich aber bei keiner anderen Baumart ein durchweg positiver Einfluss der Hydrogel-Beigabe auf Anwuchs, Vitalität und Zuwachs feststellen. Die Schwarzerle wies eine höhere Anwuchsrate, eine bessere durchschnittliche Vitalität und einen höheren Zuwachs auf, der allerdings nicht signifikant war, wenn Hydrogel bei der Pflanzung appliziert wurde. Für die anderen Baumarten lässt sich die Hydrogel-Anwendung bei den gegebenen Klima- und Standortsverhältnissen wie in 
Gütersloh nicht empfehlen, da insgesamt keine überzeugenden positiven Effekte erzielt wurden. 


\section{Schlussfolgerungen}

\section{Baumartenwahl}

Weiden (Salix viminalis) haben den Vorteil einer fast $100 \%$ igen Anwuchsrate mit hohen Zuwachsleistungen bei einem Ernterhythmus von 3-5 Jahren (Mini-Rotation).

Pappelklone profitieren von einer intensiven Bodenvorbereitung und reagieren darauf mit hohen Anwuchs- und Zuwachsraten in Midi-Rotation (Umtriebszeit von 4-6, max. 10 Jahre).

Für Kurzumtriebsplantagen eher untypische Baumarten wie Linde und Bergahorn, die sich nicht vegetativ vermehren lassen und nicht so wuchskräftig wie Hochleistungsklone der Weiden und Pappeln sind, verursachen zwar höhere Pflanzkosten, haben aber bei standortgerechter Baumartenwahl auch ohne kostenintensive Pflegemaßnahmen hohe Anwuchsraten und befriedigende Zuwachsleistungen in Umtriebszeiten von 10 bis 20 Jahren (Maxi-Rotation).

\section{Flächenvornutzung}

Der Anwuchserfolg und Zuwachs der Gehölze ist auf der ehemaligen Ackerfläche signifikant höher als auf der Grünlandfläche. Die Grasvegetation auf der Grünlandfläche stellt ohne flächige Bodenbearbeitung eine zu hohe Konkurrenzkraft für die jungen Bäume und insbesondere die Stecklinge dar, wodurch der Anwuchs und Zuwachs der Pflanzen gehemmt wird. Insbesondere die Pappelstecklinge können für Grünlandflächen mit konkurrenzkräftiger Vegetationsdecke nicht empfohlen werden. Ohne intensive Bodenbearbeitung sollte auf bewurzelte 1-2 jährige Baumschulpflanzen zurückgegriffen werden. Ein weiteres Problem auf Grünlandflächen stellt die Gefahr von Mäusepopulationen dar. Eine dichte Grasdecke fördert deren Verbreitung, was zu hohen Ausfällen und kostenintensiven Bekämpfungsmaßnahmen und Nachpflanzungen führen kann. Auf Grünlandflächen isł also eine intensive Bodenbearbeitung vor der Kulturanlage zu empfehlen. 


\section{Flächenvorbereitung}

Eine intensive Bodenbearbeitung vor Flächenbegründung verringert den Konkurrenzdruck der Begleitvegetation und erhöht den Anwuchserfolg und Zuwachs der Gehölze. Der Einsatz von Herbiziden war für eine ausreichende Eindämmung der Begleitvegetation und erfolgreiche Flächenanlage nicht erforderlich.

Das Pflügen der Fläche ließ den Nitrataustrag mit dem Sickerwasser impulsartig ansteigen. Dieser sank innerhalb eines halben Jahres unter den erlaubten Grenzwert $a b$ und blieb durch eine daverhafte Bodenruhe konstant niedrig. Kurzumtriebsplantagen reduzieren den durchschnittlichen Nitrataustrag mit dem Sickerwasser im Vergleich zu traditionell bewirtschafteten landwirtschaftlichen Flächen. Nur streifenweise gepflügte Pflanzreihen wurden rasch wieder von der Konkurrenzvegetation zugewachsen und führten zu geringeren Anwuchsraten und Zuwächsen der Gehölze. Auf Grünlandflächen empfiehlt sich für die Eindämmung der Konkurrenzvegetation eine flächige Bodenvorbereitung. Ein nur streifenweises Pflügen ist auch aufgrund erhöhter Wühlmausgefahr nicht zu empfehlen.

\section{Begleitwuchsregulierung}

Bei nicht allzu starkem Konkurrenzdruck der Begleitvegetation kann insbesondere bei Weidenstecklingen und bewurzelten Pflanzen auf Pflegemaßnahmen verzichtet werden. Bei Pappelstecklingen können Pflegemaßnahmen die Anwuchs- und Zuwachsraten insbesondere im Pflanzjahr erhöhen. Eine intensive Flächenvorbereitung ist entscheidender für den Kulturerfolg als die Begleitwuchsregulierung. Bei einer ausreichenden Flächenvorbereitung sind weitere Pflegemaßnahmen nicht zwingend erforderlich.

Die ausgesäten Nutzpflanzen Buchweizen und Klee stellten keine geeignete Methode der Begleitwuchsregulierung dar. Diese verdrängten zwar die natürliche Konkurrenzvegetation, aber stellten selber eine zu starke Konkurrenz für die Gehölze dar. 


\section{Bodenhilfsstoffe}

Die Bodenhilfsstoffe erzielten sehr baumartenspezifische Effekte, so dass kaum generelle Aussage über deren Wirkung getroffen werden können.

Der Bodenhilfsstoff Hydrogel konnte den Anwuchs und Zuwachs, sowie die Vitalität einzelner Gehölze verbessern. Die Schwarzerle war die einzige Baumart, die in allen Bereichen von der Hydrogel-Beigabe profitierte. Bei extremeren Klima- und Standortverhältnissen wurden in der Vergangenheit positive Effekte in Bezug auf Überlebens- und Zuwachsraten erzielt.

Die Mykorrhizierung der Gehölze mit Glomus intraradices verbesserte zwar die Nährstoffversorgung und Zuwachsraten einzelner Baumarten, Anwuchs und Vitalität wurden aber nicht bzw. negativ beeinflusst. Der positive Effekt der Mykorrhizierung ist in de vorliegenden Arbeit zu gering, um den Aufwand bei der Pflanzung zu rechtfertigen. 


\section{Zusammenfassung}

Auf drei neu angelegten Versuchsflächen in Nordwestdeutschland (Landkreis Gütersloh, Landkreis Uelzen) wurden waldbauliche Methoden zur Anlage und Bewirtschaftung von Kurzumtriebsplantagen auf ehemals landwirtschaftlich genutzten Flächen getestet.

Die Versuchsfläche bei Gütersloh (Sudheide) unterteilte sich in eine Ackerfläche, die vor der Kulturbegründung gepflügt und geeggt wurde, und eine Grünlandfläche, auf welcher die Pflanzreihen streifenweise gespritzt und gepflügt wurde. Im Frühjahr 2007 wurden auf der Grünlandfläche die Stecklinge der Pappelhybriden "Max 4" (P. nigra x P. maximowiczii) und "NE 42" (P. maximowiczii x P. trichocarpa) und der Weide "Turbo" (Salix viminalis), sowie zweijährige Aspen (Populus tremula) und Robinien (Robinia pseudoacacia) gepflanzt. Auf der Ackerfläche wurden zusätzlich zwei- bis dreijährige Pflanzen des Bergahorns (Acer pseudoplatanus), der Eberesche (Sorbus aucuparia), Schwarzerle (Alnus glutinosa) und Winterlinde (Tilia cordata) gepflanzt. Es wurden drei Varianten der Begleitwuchsregulierung (Kontrolle, Mähen, Nutzpflanzeneinsaat) und vier Varianten mit Bodenhilfsstoffen (Kontrolle, Hydrogel, Mykorrhiza, Hydrogel/Mykorrhiza) durchgeführt.

Im Landkreis Uelzen wurde 2006 die Versuchsfläche Hamerstorf mit den Pappelstecklingen "Androscoggin" (P. maximowiczii x P. trichocarpa), "Max 4" (P. nigra x P. maximowiczii), "NE 42" (P. maximowiczii x P. trichocarpa) und "Weser 6" (P. trichocarpa) bepflanzt. Vor der Kulturbegründung wurde die ehemalige Brachfläche auf drei unterschiedliche Arten vorbereitet (Pflügen/Eggen, Pflügen, Herbizide). Bei der Versuchsfläche Unterlüss (Landkreis Uelzen) handelt es sich um einen Wildacker, welcher 2008 mit den Pappelstecklingen "Max 2" (P. nigra $x$ P. maximowiczii), "Max 3" (P. nigra x P. maximowiczii), "NE 42" (P. maximowiczii x P. trichocarpa) und "Weser 6" (P. trichocarpa) bepflanzt wurde. Ein Teil der Pappelstecklinge wurde bei der Pflanzung mykorrhiziert. 
In den Vegetationsperioden 2007 und 2008 wurden auf den drei Versuchsflächen die Überlebens- und Zuwachsraten der jungen Bäume gemessen sowie Vegetationsaufnahmen durchgeführt. Zusätzlich wurden auf der Versuchsfläche Sudheide die Nährstoffversorgung der Gehölze, Stoffausträge mit dem Sickerwasser und Bodensaugspannung ermittelt.

Ziel der Abreit war es aufgrund der Datenerhebungen aus den ersten Jahren nach der Kulturanlage Handlungsempfehlungen für die Anlage und Pflege von Energieholzplantagen geben zu können.

Die Ergebnisse zeigten, dass bei relativ geringem Konkurrenzdruck der Begleitvegetation und angepassten Baumarten keine Maßnahmen zur Begleitwuchsregulierung notwendig waren. Vor allem Weide hatte fast $100 \%$ ige Anwuchsraten vorzuweisen. Mit Ausnahme der Schwarzerle, für die der Standort Gütersloh zu trocken war, wiesen auch die Baumschulpflanzen gute Anwuchs- und Zuwachsraten auf. Das Mähen zwischen den Pflanzreihen förderte den Anwuchs und Zuwachs der Gehölze nur in Einzelfällen. Die eingesäte Hilfspflanzendecke aus Buchweizen und Klee war als Begleitwuchsregulierung ungeeignet. Zwar konnte die eingesäten Nutzpflanzen die natürliche Begleitvegetation eindämmen, stellten aber selber für die Gehölze eine zu hohe Konkurrenzkraft um Nährstoffe und Wasser dar. Das Aufkommen der Konkurrenzvegetation wurde mehr von der Flächenvorbereitung als von der Begleitwuchsregulierung beeinflusst. Unabhängig von der Begleitwuchsregulierung waren Anwuchs- und Zuwachsraten der Gehölze auf der flächig gepflügten Ackerfläche signifikant höher als auf der nur streifenweise gepflügten Grünlandfläche. Die bei Kulturbegründung vegetationsfreien Pflanzstreifen wurden auf der Grünlandfläche sehr schnell wieder von der umgebenden Vegetation bewachsen, was zu hohen Ausfällen der Pappelstecklinge führte. Je intensiver der Eingriff ins Bodengefüge war, desto geringer war der Konkurrenzdruck der natürlichen Begleitvegetation und desto höher waren Überlebensraten und Höhenzuwächse der Pappelhybriden. Eine Herbizidbehandlung war für eine ausreichende Verdämmung der Begleitvegetation nicht notwendig. Der Nachteil einer mechanischen 
Bodenbearbeitung war der durch die erhöhte Mineralisation verursachte Nitrataustrag. Die Bodenbearbeitung erhöhte den Nitrataustrag mit dem Sickerwasser schlagartig. Die andauernde Bodenruhe auf den Kurzumtriebsplantagen ließ den Nitrataustrag unter den gesetzlich erlaubten Grenzwert absinken und im Gegensatz zu landwirtschaftlich genutzten Flächen konstant niedrig bleiben.

Die angewendeten Bodenhilfsstoffe Hydrogel und Mykorrhiza beeinflussten den Anund Zuwachs der Gehölze sehr Baumarten spezifisch. In vorhergegangenen Studien konnte Hydrogel auf Standorten, die unter extremer Sommertrockenheit leiden, die Anwuchs- und Zuwachsraten von Gehölzen deutlich verbessern. Auf dem eher moderaten Standort Gütersloh verbesserte Hydrogel bei einzelnen Baumarten die Vitalität der Gehölze, hatte aber keinen Einfluss auf Anwuchs und Zuwachs der jungen Bäume. Die Mykorrhizierung der Gehölze bei der Pflanzung konnte die Nährstoffversorgung und Zuwächse einzelner Baumarten verbessern. 


\section{Summary}

At three study sites in Northwestern Germany different methods of planting and managing short rotation coppices on former arable land were tested.

One study site (Gütersloh) was divided into former arable land, which was ploughed and harrowed before planting, and into former grassland, at which the planting rows were treated with herbicides and ploughed. In spring 2007 cuttings of poplar hybrids "Max 4" (P. nigra x P. maximowiczii), "NE 42" (P. maximowiczii x P. trichocarpa), cuttings of the willow "Turbo" (Salix viminalis) and two year old bare rooted plants of Populus tremula and Robinia pseudoacacia were planted at the former grassland. At the former arable land two to three year old bare rooted plants of Acer pseudoplatanus, Sorbus aucuparia, Alnus glutinosa and Tilia cordata were also planted. Three methods of vegetation management (control, mowing, competitive replacement) and four methods of applying soil additives (control, hydrogel, mycorrhizae, hydrogel/mycorrhizae) were tested.

The second study site (Hamerstorf) was planted in 2006 with cuttings of the poplar hybrids "Androscoggin" (P. maximowiczii x P. trichocarpa), "Max 4" (P. nigra $x$ P. maximowiczii), "NE 42" (P. maximowiczii x P. trichocarpa) and "Weser 6" (P. trichocarpa). Before planting the former fallow ground was prepared in three different ways (ploughing/harrowing, ploughing, herbicides).

The third study site (Unterlüß) had been managed as a wildlife food plot before planting the poplar cuttings "Max 2" (P. nigra x P. maximowiczii), "Max 3" (P. nigra $x$ P. maximowiczii), "NE 42" (P. maximowiczii x P. trichocarpa) and "Weser 6" (P. trichocarpa) in 2008. One part of the poplar cuttings was treated with mycorrhizae before planting.

In 2007 and 2008 the survival as well as the growth of the young trees was measured and the species composition as well as the biomass of the ground vegetation was assessed. Additionally the nutritional supply of the trees, the element outflow in the leakage water and the suction power was determined. 
The aim of this thesis was to make recommendations for the planting and management of a short rotation coppices based on the data determined.

The results showed that no management of vegetation is necessary when the biomass production of the ground vegetation is low and the tree species were adapted. Especially willow had an almost $100 \%$ survival rate. With the exception of Alnus glutinosa the rooted plants had also satisfying survival and growth rates. Mowing between the planting rows had a positive effect on survival and growth only in exceptional cases. Sowing buckwheat and clover as competitive species was not qualified as vegetation management. It reduced the biomass of the natural vegetation, but was itself a too high competition in nutrition and water. The biomass of ground vegetation was most influenced by the preparation of soil. Results clarified that only preparing the planting rows was not as effective as the preparation of the whole planting ground. The prepared planting rows became overgrown by the ground vegetation very fast, which reduced the survival of the trees, especially of the poplar cuttings. The more intensive the ground preparation was, the higher was the survival and growth of the young poplar. The chemical preparation with herbicides was not necessary for an adequate suppression of the weed. The negative effect of a mechanical preparation was a high nitrate leakage caused by the higher mineralisation. The mechanical treatment increased the nitrate leakage exponentially. Not treating the soil within the rotation cycle let the nitrate leakage sink under the legal limit so that it was constantly lower than the nitrate leakage of annually cultivated agricultural land.

The soil additives hydrogel and mycorrhizae effected the survival and growth of specific trees. Especially on dry study sites hydrogel increased the survival and growth of trees in former studies. At the climatic more moderate study site hydrogel increased the vitality of specific tree species, but it had no effect at the survival and growth of the trees. The mycorrhization of the trees increased the nutritional supply and growth of some specific tree species. 


\section{Verzeichnisse}

\subsection{Abbildungen}

Abb. 3.1: Lage der Versuchsflächen in Deutschland (Quelle: Google Earth) 23

Abb. 3.2: Schematischer Aufbau der Versuchsfläche Sudheide in Gütersloh 25

Abb. 3.3: Parzellenaufbau je Baumart auf der Versuchsfläche Sudheide in Gütersloh

Abb. 3.4: Flächenaufbau in Hamerstorf (Uelzen) ................................................ 32

Abb. 3.5: Flächenaufbau in Unterlüß (Uelzen) ................................................. 34

Abb. 4.1: Temperaturverlauf und Niederschlagsmengen im Wasserwirtschaftsjahr 2007 (Nov.06 - Okt.07) (Quelle: SWG).

Abb. 4.2: Temperaturverlauf und Niederschlagsmengen im Wasserwirtschaftsjahr 2008 (Nov.07 - Okt.08) (Quelle: SWG). 44

Abb. 4.3: Entwicklung des Austrages an Nitrat mit dem Sickerwasser nach der mechanischen Bearbeitung von Acker und Grünland im Nov. 2006 im Vergleich zu einer unbearbeiteten Grünfläche

Abb. 4.4: Nitrataustrag mit dem Sickerwasser zwischen den im März 2007 gepflanzten Weidenstecklingen in Abhängigkeit von der Begleitwuchsregulierung (Kontrolle; Mähen; Nutzpflanzeneinsaat).

Abb. 4.5: Bodensaugspannung auf $\operatorname{der} \operatorname{Acker-}(A)$ und Grünlandfläche $(G)$ in den Bodentiefen (10, 30 und $70 \mathrm{~cm}$ ) in Abhängigkeit von der Begleitwuchsregulierung (K: Kontrolle; M: Mähen; N: Nutzpflanzeneinsaat) auf der Versuchsfläche Sudheide in Gütersloh

Abb. 4.6: Verlauf der Temperatur und Bodensaugspannung in den Jahren 2007 und 2008 auf der Versuchsfläche Sudheide in Gütersloh.... 50

Abb. 4.7: Trockenmasse $\left(\mathrm{g} / \mathrm{m}^{2}\right)$ der natürlichen Begleitvegetation (ein-, zweikeimblättrige Kräuter) und der eingesäten Nutzpflanzen (Buchweizen, Klee) in der ersten Vegetationsperiode (Juli 2007) in Abhängigkeit von der 
Begleitwuchsregulierung auf der Acker- und Grünlandfläche in Gütersloh $( \pm$

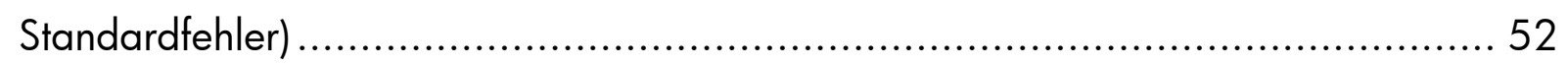

Abb. 4.8: Trockenmasse $\left(\mathrm{g} / \mathrm{m}^{2}\right)$ der natürlichen Begleitvegetation (ein-, zweikeimblättrige Kräuter) und der eingesäten Nutzpflanzen (Buchweizen, Klee) in der zweiten Vegetationsperiode (Juli 2008) in Abhängigkeit von der Begleitwuchsregulierung auf der Acker- und Grünlandfläche in Gütersloh ( \pm

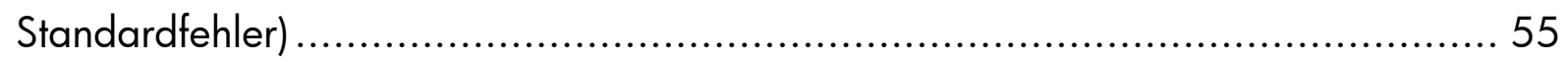

Abb. 4.9: Anwuchsprozente der Pappelstecklinge in der ersten Vegetationsperiode in Abhängigkeit von der Trockenbiomasse der einkeimblättrigen Kräuter 63

Abb. 4.10: Prozentuale Anteile an vitalen Aspen und Robinien in der ersten Vegetationsperiode in Abhängigkeit von der Trockenbiomasse der einkeimblättrigen Kräuter 64

Abb. 4.11: Überlebensrate von Aspe und Robinie nach der zweiten Vegetationsperiode in Abhängigkeit vom Deckungsgrad der Begleitvegetation auf der Versuchsfläche Sudheide in Gütersloh. 65

Abb. 4.12: Durch Mäusefraß verursachte Pflanzenausfälle auf der Acker- und Grünlandfläche Sudheide in Gütersloh nach zwei Vegetationsperioden. 66 Abb. 4.13: Höhenzuwachs der Kontrollpflanzen in der ersten Vegetationsperiode 2007 auf der Versuchsfläche Sudheide bei Gütersloh ( \pm Standardabweichung) ...... 67 Abb. 4.14: Höhenzuwachs der Gehölze auf der Versuchsfläche Sudheide in den ersten beiden Vegetationsperioden..... 68

Abb. 4.15: Höhenzuwachs der Pappelstecklinge in der ersten Vegetationsperiode in Abhängigkeit von der Trockenbiomasse der einkeimblättrigen Kräuter 74 Abb. 4.16: Regression des Höhenzuwachses des 2-jährigen Pappelstecklings "Max 4" mit der P- und K-Konzentration in der Blatt-Trockenmasse Auf der Acker- und Grünlandfläche Gütersloh 78 
Abb. 4.17: Regression des Höhenzuwachses des 2-jährigen Pappelstecklings "NE 42" mit den P-, K- und Ca-Konzentrationen in der Blatt-Trockenmasse auf der Acker- und Grünlandfläche in Gütersloh 78

Abb. 4.18: Regression des Höhenzuwachses der Aspe und Robinie in zwei Vegetationsperioden mit der K-Konzentration in der Blatt-Trockenmasse auf der Ackerund Grünlandfläche in Gütersloh 79

Abb. 4.19: Regression des Höhenzuwachses des mykorrhizierten und nicht mykorrhizierten Pappelstecklings "Max 4" in zwei Vegetationsperioden mit den P- und Ca-Konzentrationen in der Blatt-Trockenmasse auf der Ackerfläche in Gütersloh 81

Abb. 4.20: Trockenmasse $\left(\mathrm{g} / \mathrm{m}^{2}\right)$ der Bodenvegetation in der 2 . und 3 . Vegetationsperiode auf der Versuchsfläche Uelzen in Abhängigkeit von der Flächenvorbereitung 83

Abb. 4.21: Überlebensraten der unterschiedlichen Pappelhybriden auf der geeggten Versuchsfläche Hamerstorf nach der 2. und 3. Vegetationsperiode 84

Abb. 4.22: Überlebensrate des Pappelhybriden "NE 42" nach der 2 . und 3. Vegetationsperiode in Abhängigkeit von der Flächenvorbereitung auf der Versuchsfläche Hamerstorf. 85

Abb. 4.23: Pappelhöhen nach der 2. und 3. Vegetationsperiode auf der geeggten Versuchsfläche Hamerstorf. 86

Abb. 4.24: Höhe des Pappelhybriden "Ne 42" nach der 2 . und 3. Vegetationsperiode in Abhängigkeit von der Vorbereitung der Versuchsfläche Hamerstorf 88

Abb. 4.25: Anwuchsraten der Pappelhybride in der ersten Vegetationsperiode auf dem Wildacker in Unterlüß in Abhängigkeit von einer Mykorrhizierung 89 Abb. 4.26: Höhe der Pappelhybriden auf dem Wildacker in Unterlüß nach der ersten Vegetationsperiode in Abhängigkeit von einer Mykorrhizierung 90 


\subsection{Tabellen}

Tab. 2.1: Eigenschaften von wichtigen Pappelarten für den Kurzumtrieb (Röhricht \&

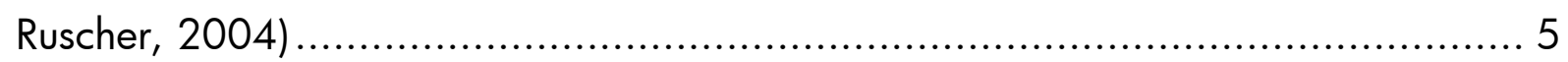

Tab. 2.2: Biomasseleistung der wichtigsten Pappelarten und Kreuzungen bei einer Umtriebszeit von vier Jahren und guten Wuchsbedingungen (Hofmann, 2007)......... 6 Tab. 2.3: Eigenschaften von Weidenarten für den Kurzumtrieb (Röhricht \& Ruscher, 2004). 7

Tab. 2.4: Für häufig vorkommende Böden geeignete Baumarten (Schlüter, 1990) ... 12 Tab. 2.5: Wichtige Weidensorten für den Kurzumtrieb (Röhricht \& Ruscher, 2004) . 14 Tab. 2.6: Wichtige Pappelsorten für den Kurzumtrieb (Röhricht \& Ruscher 2004) ... 16 Tab. 3.1: Auf der Fläche Sudheide verwendetes Pflanzenmaterial 26

Tab. 3.2: Standraumgrößen und Pflanzenzahlen/ha auf der Fläche Sudheide (Gütersloh) 26

Tab. 3.3: Saatgutmischung für die Nutzpflanzendecke auf der Fläche Sudheide (Gütersloh) 28

Tab. 3.4: Auf der Versuchsfläche Hamerstorf bei Uelzen gepflanzte Pappelstecklinge 32

Tab. 3.5: Auf der Versuchsfläche Unterlüß bei Uelzen gepflanzte Pappelstecklinge.. 34 Tab. 4.1: Monatliche Durchschnittstemperaturen und Niederschläge für Gütersloh (1961 - 1990) und der Wasserwirtschaftsjahre 2007 (Nov.'06 - Okt.'07) und 2008 (Nov.'07 - Okt.'08) (Quellen: DWD; SWG)

Tab. 4.2: Hauptpflanzenarten der Begleitvegetation auf der Versuchsfläche Sudheide in Gütersloh. 51

Tab. 4.3: Anwuchserfolg (\%) der Gehölze auf der Acker- und Grünlandfläche in der ersten Vegetationsperiode in Abhängigkeit von der Begleitwuchsregulierung ( \pm Standardfehler; * signifikante Mittelwert-Differenz zur Kontrolle mit $p \leq 0,05)$....... 56 
Tab. 4.4: Überlebensrate (\%) der Gehölze auf der Acker- und Grünlandfläche nach der zweiten Vegetationsperiode in Abhängigkeit von der Begleitwuchsregulierung ( \pm Standardfehler; * signifikante Mittelwert-Differenz zur Kontrolle mit $p \leq 0,05)$....... 57 Tab. 4.5: Anwuchs- und Überlebensraten der Gehölze auf der Acker- und Grünlandfläche in der ersten ('07) und zweiten ('08) Vegetationsperiode in Abhängigkeit von den verwendeten Bodenhilfsstoffen (*signifikanter Unterschied zur Kontrolle mit $p \leq 0,05)$. 58

Tab. 4.6: Mittlere Vitalität der lebenden Gehölze auf der Acker- und Grünlandfläche in der ersten Vegetationsperiode in Abhängigkeit von den verwendeten Bodenhilfsstoffen ( 1 vital; 4 fast völlig abgestorben; \pm Standardfehler; * signifikante Mittelwert-Differenz zur Kontrolle mit $p \leq 0,05$ ) 60

Tab. 4.7: Mittlere Vitalität der lebenden Gehölze auf der Acker- und Grünlandfläche in der zweiten Vegetationsperiode in Abhängigkeit von den verwendeten Bodenhilfsstoffen ( 1 vital; 4 fast völlig abgestorben; \pm Standardfehler; * signifikante Mittelwert-Differenz zur Kontrolle mit $p \leq 0,05$ ) 61

Tab. 4.8: Höhenzuwachs (cm) der Gehölze auf der Acker- und Grünlandfläche in der ersten Vegetationsperiode in Abhängigkeit von der Begleitwuchsregulierung $( \pm$ Standardfehler; * signifikante Mittelwert-Differenz zur Kontrolle mit $p \leq 0,05$ )

Tab. 4.9: Gesamthöhenzuwächse der Gehölze auf der Versuchsfläche Sudheide nach zwei Vegetationsperioden in Abhängigkeit von der Begleitwuchsregulierung $( \pm$ Standardfehler; * signifikante Mittelwert-Differenz zur Kontrolle mit $p \leq 0,05) \ldots \ldots . .70$ Tab. 4.10: mittlere Höhenzuwächse der Gehölze auf der Versuchsfläche Sudheide nach der ersten Vegetationsperiode in Abhängigkeit von den applizierten Bodenhilfsstoffen ( \pm Standardfehler; * signifikante Mittelwert-Differenz zur Kontrolle mit $\mathrm{p} \leq 0,05)$ 71

Tab. 4.11: Gesamthöhenzuwächse der Gehölze auf der Versuchsfläche Sudheide nach zwei Vegetationsperioden in Abhängigkeit von den applizierten Bodenhilfsstoffen ( \pm Standardfehler; * signifikante Mittelwert-Differenz zur Kontrolle mit $p \leq 0,05) \ldots .73$ 
Tab 4.12: Optimale Blattspiegelwerte nach van den Burg (1990) (k.A. - keine Angaben). 75

Tab. 4.13: Nährstoffkonzentrationen der gepflanzten Gehölze auf Acker- und Grünlandfläche in Gütersloh in der zweiten Vegetationsperiode 76

Tab. 4.14: Nährstoffkonzentrationen von Bergahorn, Pappel "Max 4" und Weide "Turbo" auf der Ackerfläche in Gütersloh in der zweiten Vegetationsperiode in Abhängigkeit von der Begleitwuchsregulierung (Mähen; Nutzpflanzen) und den applizierten Bodenhilfsstoffen (Hydrogel, Mykorrhiza; Hydrogel + Mykorrhiza) ( ${ }^{*}$ signifikante Mittelwert-Differenz zur Kontrolle mit $\left.p \leq 0,05\right)$. 80

Tab. 4.15: Artenliste der biomassebildenden Begleitvegetation auf der Fläche Hamerstorf in der 2. (2007) und 3. Vegetationsperiode (2008) 82

Tab. 4.16: Artenliste der biomassebildenden Begleitvegetation auf der Versuchsfläche Unterlüß in der ersten Vegetationsperiode 2007 (DG-Deckungsgrad; TM Trockenmasse; \pm Standardabweichung Trockenmasse) 89

\subsection{Datenquellen}

\section{Klimadaten:}

Deutscher Wetterdienst (DWD)

Stadtwerke Gütersloh GmbH (SWG), Gütersloh, Germany

\section{Produktinformationen:}

AMykor GmbH, Wolfen, Germany

Deutsche Saatveredelung AG, Springe Lüdersen, Germany

Gefa Produkte Fabritz GmbH, Krefeld, Germany

Monsanto Agrar Deutschland GmbH, Düsseldorf, Germany 


\section{Satellitenbilder:}

Google Earth 5.0.1

\subsection{Literatur}

Akhter, J., Mahmood, K., Malik, K. A., Mardan, A., Ahmad, M. \& IQbal, M. M. (2004). Effects of hydrogel amendment on water storage of sandy loam and loam soils and seedling growth of barley, wheat and chickpea. - Plant Soil Environ. 50, 463-469.

Al-Humaid, A. I. \& MOFTAH, A. E. (2007). Effects of hydrophilic polymer on the survival of buttonwood seedlings grown under drought stress. - Journal of Plant Nutrition 30, 53-66.

Allen, E. B., Allen, M. F., Egerton-Warburton, L., Corkidi, L. \& Gomez-Pompa, A. (2003). Impacts of early- and late-seral mycorrhizae during restoration in seasonal tropical forest, Mexico. - Ecological Applications 13, 1701-1717.

ANTON, K. (1999). Aufforstung landwirtschaftlicher Nutzflächen als Beitrag zum Trinkwasserschutz im Wasserschutzgebiet Holdorf. - Forst und Holz 54, 404408.

BAUM, C., SCHMID, K. \& MAKESCHIN, F. (2000). Interactive effects of substrates and ectomycorrhizal colonization on growth of a poplar clone. - Journal of Plant Nutrition and Soil Science 163, 221-226.

Baum, C., Stetter, U. \& MAKeschiN, F. (2002). Growth response of Populus trichocarpa to inoculation by the ectomycorrhizal fungus Laccaria laccata in a pot and a field experiment. - Forest Ecology and Management 163, 1-8.

BELLOCQ, M. I. \& SMITH, S. M. (1995). Influence of reforestation technique, slash, competing vegetation, and duff depth on the overwintering mortality of Pissodes strobi (Coleoptera: Curculionidae), the white pine weevil. - Forest Ecology and Management 78, 1-10.

BensON, M. L., MYers, B. J. \& RaISON, R. J. (1992). Dynamics of stem growth of Pinus radiata as affected by water and nitrogen supply. - Forest Ecology and Management 52, $117-137$.

BERMAN, J. T. \& BLEDSOE, C. S. (1998). Soil transfers from valley oak (Quercus lobata Nee) stands increase ectomycorrhizal diversity and alter root and shoot growth on valley oak seedlings. - Mycorrhiza 7, 223-235.

BLASCHKE, H. (1980). Zur Mykorrhizaforschung bei Waldbäumen. Forstwissenschaftliches Centralblatt 99, 6-12.

BlOCK, R. M. A., KNIGHT, J. D., BOOTH, N. W. H. \& ReEs, K. C. J. V. (2009). Nursery stock type, nitrogen fertilization and shoot pruning effects on the growth of 
juvenile hybrid poplar in Saskatchewan. - Canadian Journal of Plant Science 89, 289-301.

BOELCKE, B. (2006). Schnellwachsende Baumarten auf landwirtschaftlichen Flächen: Leitfaden zur Erzeugung von Energieholz. - Ministerium für Ernährung Landwirtschaft Forsten und Fischerei Mecklenburg-Vorpommern, Schwerin, 40.

BUCHNER, W. (1987). So konservieren Sie Nitrat im Boden. - DLG-Mitteilungen 102, 518-523.

BUNGART, R. \& HUTTL, R. F. (2004). Growth dynamics and biomass accumulation of 8year-old hybrid poplar clones in a short-rotation plantation on a clayey-sandy mining substrate with respect to plant nutrition and water budget. - European Journal of Forest Research 123, 105-115.

Burger, F. (2004). Technologie und Ökonomie des Anbaus und der Ernte von Feldholz. - Energieholzproduktion in der Landwirtschaft, Bornimer Agrartechnische Berichte, 61-73.

BURGER, F. (2006). Experiences with short rotation forests in Bavaria: Bayerische Erfahrungen bei der Bewirtschaftung von Kurzumtriebswäldern. - Forst und Holz 61, 484-486.

Coll, L., BALANDIER, P. \& PiCON-COChARD, C. (2004). Morphological and physiological responses of beech (Fagus sylvatica) seedlings to grass-induced belowground competition. - Tree physiology 24, 45-54.

Coll, L., Messier, C., Delagrange, S. \& Berninger, F. (2007). Growth, allocation and leaf gas exchanges of hybrid poplar plants in their establishment phase on previously forested sites: effect of different vegetation management techniques. - Annals of Forest Science 64, 275-285.

Craven, D., Hall, J. \& Verjans, J. M. (2009). Impacts of herbicide application and mechanical cleanings on growth and mortality of two timber species in Saccharum spontaneum grasslands of the Panama Canal Watershed. Restoration Ecology 17, 751-761.

CZAPOWSKYJ, M. M. \& SAFFORD, L. O. (1993). Site preparation, fertilization, and 10year yields of hybrid poplar on a clearcut forest site in eastern Maine, USA. New Forests 7, 331-344.

DAVIES, R. J. (1985). The Importance of Weed Control and the Use of Tree Shelters for Establishing Broadleaved Trees on Grass-Dominated Sites in England. Forestry 58, 167-180.

Davis, M. A., Wrage, K. J., Reich, P. B., TJoelker, M. G., Schaeffer, T. \& MuermanN, C. (1999). Survival, growth, and photosynthesis of tree seedlings competing with herbaceous vegetation along a water-light-nitrogen gradient. - Plant Ecology 145, 341-350.

DELL, B. (2002). Role of Myccorhizal Fungi in Ecosystems. - CMU. Journal 1, 47-60.

DENECKE, F. (1988). Tending young growth and cleaning up the site using ground covers of useful plants: Jungwuchspflege und Standortsanierung durch Nutzpflanzendecken. - Allgemeine Forstzeitschrift, 221-222. 
DIEDERICHS, W. (1990). Zur Anbautechnik von Schnellwuchsplantagen. Bundesforschungsanstalt für Forst- und Holzwirtschaft Institut für Ökonomie, Hamburg.

DIMITRI, L. (1988). Bewirtschaftung schnellwachsender Baumarten im Kurzumtrieb zur Energiegewinnung. - Forschungsinst. für schnellwachsende Baumarten, Hann.Münden.

Don, A., Arenhövel, W., JaCob, R., SCherer-Lorenzen, M. \& SChulze, E.-D. (2007). Anwuchserfolg von 19 verschiedenen Baumarten bei ErstaufforstungenErgebnisse eines Biodiversitätsexperiments. - Allgemeine Forst- und Jagdzeitung 178, 164-172.

DOSSKEY, M. G., LINDERMAN, R. G. \& Boersma, L. (1990). Carbon-sink stimulation of photosynthesis in Douglas fir seedlings by some ectomycorrhizas. - New Phytologist 115, 269-274.

EISENREICH, H. (1956). Schnellwachsende Holzarten: Ein Leiffaden. - Deutscher Bavernverlag, Berlin.

ESCHENBACH, C. \& KAPPEN, L. (1999). Leaf water relations of black alder [ Alnus glutinosa (L.) Gaertn.] growing at neighbouring sites with different water regimes. - Trees - Structure and Function 14, 28-38.

EVERS, J. (2001). Stoffhaushalt und Waldbautechnik bei Erstaufforstungen ehemals landwirtschaftlicher Nutzflächen. - Landesanstalt für Ökologie, Bodenordnung und Forsten Nordrhein-Westfalen, LÖBF-Schriftenreihe, Band 19, 242.

EVERS, J. \& PAMPE, A. (2005). Artificial mycorrhizas of forest nursery plants. Studies to improve survival of beech and sycamore in afforestation. - Forst und Holz 60, 83-90.

FANG, S., XIE, B. \& LIU, J. (2008). Soil nutrient availability, poplar growth and biomass production on degraded agricultural soil under fresh grass mulch. - Forest Ecology and Management 255, 1802-1809.

FEIL, W., KOTTKE, I. \& OBERWINKLER, F. (1988). The effect of drought on mycorrhizal production and very fine root system development of Norway spruce under natural and experimental conditions. - Plant and Soil 108, 221-231.

Ferrari, A. E. \& WAlL, L. G. (2008). Coinoculation of black locust with Rhizobium and Glomus on a desurfaced soil. - Soil Science 173, 195-202.

FitTeR, A. \& GARBAYE, J. (1994). Interactions between mycorrhizal fungi and other soil organisms. - Plant and Soil 159, 123-132.

FÜHRER, E. (2005). Robinienwirtschaft in Ungarn: I. Die Robinie im praktischen Waldbau. - Forst und Holz, 464-466.

Gakis, S., Mantzanas, K., Alifragis, D., Papanastasis, V., Papaloannou, A., SEILOPOULOS, D. \& PLATIS, P. (2004). Effects of understorey vegetation on tree establishment and growth in a silvopastoral system in northern Greece. Agroforestry Systems 60, 149-157. 
GeYer, W. A., AtChinsON, R. L. \& BARDEN, C. J. (2009). Comparison of weed control methods in Populus tree seedlings establishment. - Transactions of the Kansas Academy of Science 111, 263-268.

GRIESCHE, C., SCHMIDT, O. (2012). Die Eberesche/Vogelbeere. Bauminformationsblatt Nr.11, Herausgeber: Schutzgemeinschaft Deutscher Wald Bundesverband e.V. (SDW), http://www.sdw.de/cms/upload/pdf/Die_Eberesche.pdf (14.01.2012)

GUERICKE, M. (2006). Anlage von Schnellwuchsplantagen auf landwirtschaftlichen Nutzflächen zur Erzeugung von Energieholz. Erste Erfahrungen mit dem Anbau von Weiden im Kurzumtrieb. - Forst und Holz 61, 478-483.

GUNREBEN, M. \& BOESS, J. (2008). Schutzwürdige Böden in Niedersachsen. GeoBerichte 8 (Landesamt für Bergbau Energie und Geologie, ed), Hannover, 48.

HASelWANDTER, K. \& BOWEN, G. D. (1996). Mycorrhizal relations in trees for agroforestry and land rehabilitation. - Forest Ecology and Management 81, 117.

Hesin, K. \& Douglas, G. (1985). Mycorrhizae. - Research Report 1984, Horticulture, 42-43.

HofmanN-SChielle, C., Jug, A., MAKesChIN, F. \& Rehfuess, K. E. (1999). Short-rotation plantations of balsam poplars, aspen and willows on former arable land in the Federal Republic of Germany. I. Site-growth relationships. - Forest Ecology and Management 121, 41-55.

HOFMANN, M. (1998). Bewirtschaftung schnellwachsender Baumarten auf landwirtschaftlichen Flächen im Kurzumtrieb. - Merkblatt des Forschungsinstitutes für schnellwachsende Baumarten (Forschungsinstitut für schnellwachsende Baumarten, ed), Hann. Münden.

HOFMANN, M. (2004). Ergebnisse und Erfahrungen mit schnellwachsenden Baumarten. - Energieholzproduktion in der Landwirtschaft, Bornimer Agrartechnische Berichte, Heft 35. Institut für Agrartechnik Bornim e.V. , 33-40.

HoFMANN, M. (2007). Energieholzproduktion in der Landwirtschaft. - (Fachagentur Nachwachsende Rohstoffe e.V. (FNR), ed), Gülzow.

HütTeRMANN, A., ZOMMORODI, M. \& ReISE, K. (1999). Addition of hydrogels to soil for prolonging the survival of Pinus halepensis seedlings subjected to drought. Soil and Tillage Research 50, 295-304.

HYTÖNEN, J. \& JYLHÄ, P. (2005). Effects of competing vegetation and post-planting weed control on the mortality, growth and vole damages to Betula pendula planted on former agricultural land. - Silva Fennica 39, 365-380.

JANTZEN, G. (1989). Die Entwicklung von Bodenzustand und Wurzelsystem einer Stieleichen-Rotbuchenkultur nach Einsaat von Nutzpflanzendecken zur Begleitwuchsregulierung. - $\quad$ Diplom-Arbeit, Fachhochschule Hildesheim/Holzminden. 
JefrRIES, P., BAREA, J.M. ( 2001). Arbuscular mycorrhiza-a key component of sustainable plant-soil ecosystems. - The Mycota. IX. Fungal Associations. (B. Hock, ed). Springer, Berlin, 95-113.

Jefrries, P., Gianinazzi, S., Perotto, S., turnau, K. \& Barea, J.-M. (2003). The contribution of arbuscular mycorrhizal fungi in sustainable maintenance of plant health and soil fertility. - Biology and Fertility of Soils 37, 1-16.

Jug, A., HofmanN-SChielle, C., MakesChin, F. \& Rehfuess, K. E. (1999). Short-rotation plantations of balsam poplars, aspen and willows on former arable land in the Federal Republic of Germany. II. Nutritional status and bioelement export by harvested shoot axes : Special issue: Short rotation forestry in central and northern Europe. - Forest Ecology and Management 121, 67-83.

JYLHÄ, P., HYTÖNEN, J. \& COMEAU, P. (2006). Effect of vegetation control on the survival and growth of Scots pine and Norway spruce planted on former agricultural land: Useable science, practical outcomes, and future needs. Proceedings of the Fifth International Conference on Forest Vegetation Management, 20-24 June 2006, Corvallis, Oregon, USA. - Canadian Journal of Forest Research $36,2400-2411$.

KAHLE, P. \& BOELCKE, B. (2004). Auswirkungen des Anbaus schnellwachsender Baumarten im Kurzumtrieb auf ausgewählte Bodeneigenschaften. Energieholzproduktion in der Landwirtschaft. Inst. für Agrartechnik Bornim, Potsdam-Bornim.

Kolb, T. E., Steiner, K. C., MCCormick, L. H. \& Bowersox, T. W. (1990). Growth response of northern red-oak and yellow-poplar seedlings to light, soil moisture and nutrients in relation to ecological strategy. - Forest Ecology and Management 38, 65-78.

Koss, H. (1982). Verbreitung, ökologische Ansprüche und waldbauliche Verwendung der Winterlinde (Tilia cordata Mill.). - Der Forst- und Holzwirt 37, 381-385.

KOUPAI, J., ESLAMIAN, S. \& KAZEMI, J. (2008). Enhancing the available water content in unsaturated soil zone using hydrogel, to improve plant growth indices. Ecohydrology and Hydrobiology 8, 67-75.

LAMERSDORF , N. (2007). Teilprojekt Bodenökologie - NOVALIS. Zur naturverträglichen Produktion von Energieholz in der Landwirtschaft Parametrisierung von standorts- und raumbezogenen Indikatoren zur Optimierung von Bewirtschaftungsstrategien. 1. Zwischenbericht. Forschungszentrum Waldökosysteme der Universität Göttingen (FZW), Göttingen, 134.

LAMERSDORF, N. (2008). Teilprojekt Bodenökologie - NOVALIS. Zur naturverträglichen Produktion von Energieholz in der Landwirtschaft. Parametrisierung von standorts- und raumbezogenen Indikatoren zur Optimierung von Bewirtschaftungsstrategien. 2. Zwischenbericht. Forschungszentrum Waldökosysteme der Universität Göttingen (FZW), Göttingen, 147. 
LANDESANSTALT FÜR ÖKOLOGIE LANDSCHAFTSENTWICKLUNG UND FORSTPLANUNG NRW. (1984). Robinia pseudoacacia L. - Robinie. - Merkblatt über fremdländische Baumarten.

LANDGRAF, D., BÖCKER, L. \& OldenbuRG, C. (2007). Praxisrelevante Ernte einer Kurzumtriebsplantage: Landwirte als Energieholz-Produzenten?! - AFZ/Der Wald, Allgemeine Forst Zeitschrift für Waldwirtschaft und Umweltvorsorge, 751-753.

LANDHÄUSSER, S. M. \& LIEFFERS, V. J. (1998). Growth of Populus tremuloides in association with Calamagrostis canadensis. - Can. J. For. Res. 28 (3), 396401

LEIBUNDGUT, H. (1953). Beobachtungen über den Streuabbau einiger Baumarten im Lehrwald ETH. - Schweiz. Zeitschr. für Forstwesen, 179.

LIEBHARD, P. (2007). Energieholz im Kurzumtrieb: Rohstoff der Zukunft. - Stocker, Graz.

LIESEBACH, M., WUeHLISCH, G. \& MUHS, H.-J. (1999). Aspen for short-rotation coppice plantations on agricultural sites in Germany: Effects of spacing and rotation time on growth and biomass production of aspen progenies. - Forest Ecology and Management 121, 25-39.

LIN, W., MA, H., ZHOU, J., ZHANG, Z. \& GAN, Y. (2004). Effects of hydrogel on the growth of seedlings and their physiological properties under drought stress. Ganhanqu Yanjiu (Arid Zone Research) 21, 353-357.

LÖF, M. \& WELANDER, N. T. (2004). Influence of herbaceous competitors on early growth in direct seeded Fagus sylvatica L. and Quercus robur L. - Annals of Forest Science 61, 781-788.

LÜDEMANN, G. (1993). Anlage und Pflege von Erstaufforstungen. - AFZ 5, 211 -214.

LUO, Z., LI, K., JIANG, X. \& POLLE, A. (2009). Ectomycorrhizal fungus (Paxillus involutus) and hydrogels affect performance of Populus euphratica exposed to drought stress. - Ann. For. Sci. 66, 106.

Maldonado, J. D., Tainter, F. H., SkipPer, H. D. \& Lacher, T. E. (2000). Arbuscular mycorrhiza inoculum potential in natural and managed tropical montane soils in Costa Rica. - Tropical Agriculture 77, 27-32.

MARTYN, W. \& SZOT, P. (2001). Influence of superabsorbents on the physical properties of horticultural substrates. - International Agrophysics 15, 87-94.

MARX, D. H., MARRS, L. F. \& CORdell, C. E. (2002). Practical use of the mycorrhizal fungal technology in forestry, reclamation, arboriculture, and horticulture. Dendrobiology 47, 27-40.

Meyer-Marquart, D., FeldWisCH, N. \& LendVaczky, T. (2006). Vorstudie Rahmenbedingungen und Potenziale für eine natur- und umweltverträgliche energetische Nutzung von Biomasse im Freistaat Sachsen. - Sächsisches Landesamt für Umwelt und Geologie, Dresden.

MinOtTA, G., CANTONI, L., TONON, G. \& BAGNARESI, U. (2001). Effects of grass and legume species on early growth of sycamore and wild cherry in silvopastoral 
system of the northern Apennines (Italy). - In: Proceedings International Conference Forest Research: a challenge for an integrated European approach, Thessaloniki, Greece, p. 661-666.

MORRISON, S. J., NICHOLL, P. A. \& HICKLENTON, P. R. (1993). VA mycorrhizal inoculation of landscape trees and shrubs growing under high fertility conditions. - Journal of Environmental Horticulture 11, 64-71.

MunRo, R. C., Wilson, J., JeFWA, J. \& MBUthiA, K. W. (1999). A low-cost method of mycorrhizal inoculation improves growth of Acacia tortilis seedlings in the nursery. - Forest Ecology and Management 113, 51-56.

Nilsen, P., BØRJA, I., KNUTSEN, H. \& BREAN, R. (1998). Nitrogen and drought effects on ectomycorrhizae of Norway spruce [Picea abies L.(Karst.)]. - Plant and Soil $198,179-184$.

NIISSON, U. \& ÖRLANDER, G. (1999). Vegetation management on grass-dominated clearcuts planted with Norwy spruce in southern Sweden. - Canadian Journal of Forest Research 29, 1015-1026.

Odermatt, O. \& WASEM, U. (2004). Mäuseschäden bei der Wiederbewaldung von Windwurfflächen. - http://www.waldwissen.net (04.08.2004).

OliveirA, R. S., CASTRO, P. M. L., DODD, J. C. \& VosÁtKA, M. (2005). Synergistic effect of Glomus intraradices and Frankia spp. on the growth and stress recovery of Alnus glutinosa in an alkaline anthropogenic sediment. - Chemosphere 60, 1462-1470.

Otsamo, A., Ådjers, G., Hadi, T. S., Kuusipalo, J., TuOmela, K. \& VuOKKO, R. (1995). Effect of site preparation and initial fertilization on the establishment and growth of four plantation tree species used in reforestation of Imperata cylindrica (L.) Beauv. dominated grasslands. - Forest Ecology and Management 73, 271277.

Pallast, G., Breuer, T. \& HOlM-Müller, K. (2006). Schnellwachsende Baumarten: Chance für zusätzliches Einkommen im ländlichen Raum? - Berichte über Landwirtschaft 84.

PAmpe, A., Meiwes, K. J., RÖKer, B. \& Wagner, S. (2001). Nitrate leaching following afforestation of former agricultural land. - Forst und Holz 56, 681-686.

RABANSER, N., GÖSSINGER, L. (2012). Der Bergahorn - Acer pseudoplatanus. Bauminformationsblatt, Herausgeber: Schutzgemeinschaft Deutscher Wald Bundesverband e. V. (SDW),

http://www.sdw.de/cms/upload/pdf/Der_Bergahorn.pdf (14.01.2012)

REINECKE, H. (1990). Afforestation of windthrow areas is possible under crop plants: Aufforstung von Windwurfflächen unter Nutzpflanzen möglich. - Allgemeine Forstzeitschrift, 950-955.

REISNER, Ü. (2001). Hybrid aspen and its plantations in Estonia-past, present, future. - Silviculture of Deciduous in Estonia. Proceedings of the Estonian Academical Forestry Society XIV $(\mathrm{H}$. Tullus \& A. Vares, eds), Tartu, 115-122 (in Estonian, summary in English). 
RENNER, G. (2002). Forstamt Uelzen. Waldmärkerschaft Uelzen. Brockhöfe. Standortstypenkarte. Landwirtschaftskammer Hannover. Abteilung Forstwirtschaft.

RICHTER, B. L. (1984). Orientierende Klimabeurteilung für die Gemeinde Unterlüß. Landkreis Celle. - Klimagutachten. Deutscher Wetterdienst, Hannover.

RÖHRICHT, C. (2005). Erfahrungen und Ergebnisse im Anbau schnellwachsender Baumarten im Kurzumtrieb im Freistaat Sachsen. http://www.umwelt.sachsen.de/de/wu/Landwirtschaft/Ifl/inhalt/download/V ortrag_SH_18_01_05_roehricht.pdf.

RÖHRICHT, C. \& RUSCHER, K. (2004). Anbauempfehlungen für schnellwachsende Baumarten. - Fachmaterial Sächsische Landesanstalt für Landwirtschaft.

RoWe, E. C., Williamson, J. C., Jones, D. L., Holliman, P. \& Healey, J. R. (2005). Initial Tree Establishment on Blocky Quarry Waste Ameliorated with Hydrogel or Slate Processing Fines. - Journal Environmental Quality 34, 994-1003.

Sarvas, M., PaVlenda, P. \& TaKacova, E. (2007). Effect of hydrogel application on survival and growth of pine seedlings in reclamations. - Journal of Forest Science 53, 204-209.

Savé, R., Pery, M., Marfà, O. \& Serrano, L. (1995). The effect of a hydrophilic polymer on plant water status and survival of transplanted pine seedlings. HortTechnology 5, 141-143.

SCHILDBACH, M., LANDGRAF, D. \& BÖCKER, L. (2008). Steckhölzer zur Begründung von Kurzumtriebsplantagen. - AFZ-Der Wald 18, 992-993.

SCHINDLER, U. \& MÜLLER, L. (2009). Effect of arable management practice and tillage systems on the soil water and solute balance in Northeast Germany. International Agricultural Engineering Conference, Bangkok, Thailand, 7 - 10 December, 2009

SCHLÜTER, U. (1990). Laubgehölze: Ingenieurbiologische Einsatzmöglichkeiten ; Eigenschaften Verwendungsmöglichkeiten standörtliche und bautechnische Eignung ; Laubgehölzarten für häufig vorkommende Standorte und die gebräuchlichsten ingenieurbiologischen Bauweisen. - Patzer, Berlin [u.a.].

SCHOlz, V., Hellebrand, H. J. \& HöHN, A. (2004). Energetische und ökologische Aspekte der Feldholzproduktion. - Energieholzproduktion in der Landwirtschaft. Inst. für Agrartechnik Bornim, Potsdam-Bornim, 15-32.

SCHOOF, N. (2009). Der Berg-Ahorn: Acer pseudoplatanus. Charakteristika - Ökologie - Krankheiten und Schädlinge - Nutzung. - Studienarbeit, 1. Auflage 2009, GRIN Verlag, ISBN 978-3-640-55481-2.

SCHÜLER, S., WeIBENBACHER, L. \& SIEBERER, K. (2006). Robinien für Energie- oder Wertholz - die Sorte macht's! - Forstzeitung 117, 8-9.

SChultzKe, R., LANGE, O. \& WeisGerber, H. (1990). Pappelanbau. - (Auswertungs-und Informationsdienst für Ernährung, Landwirtschaft und Forsten ed). AID, Bonn, 23. 
SCHUSTER, K. (2007). Energieholzproduktion auf landwirtschaftlichen Flächen (Kurzumtrieb, Short-Rotation Farming): Erfahrungen aus Niederösterreich. Landwirtschaftskammer Niederösterreich.

SCHWARZE, H. \& RÖHRICHT, C. (2006). Untersuchungen zum Pappel- und Weidenanbau im Kurzumtrieb auf landwirtschaftlichen Flächen. - Präsentation, Fachtagung "Anbau und Nutzung von Bäumen auf landwirtschaftlichen Flächen" in Tharandt/Sachsen am 6. und 7. November 2006.

Skousen, J., Gorman, J., Pena-YeWtukhiw, E., KIng, J., SteWart, J., Emerson, P. \& DELONG, C. (2009). Hardwood tree survival in heavy ground cover on reclaimed land in West Virginia: mowing and ripping effects. - Journal of Environmental Quality 38, 1400-1409.

SMith, S. E. \& READ, D. J. (1997). Mycorrhizal symbiosis. - Acad. Press, San Diego.

SPECHT, A. \& HARVEY-JONES, J. (2000). Improving water delivery to the roots of recently transplanted seedling trees: The use of hydrogels to reduce leaf and hasten root establishment. - Forest Reserach 1, 117-123.

Stanturf, J. A., van Oosten, C., Netzer, D. A., Coleman, M. D. \& Portwood, C. J. (2001). Ecology and silviculture of poplar plantations. - Poplar culture in North America (D. I. Dickman, ed). NRC Research Press, Ottawa, ON, 153206.

Strebel, O., Duynisveld, W. H. M. \& Böttcher, J. (1989). Nitrate pollution of groundwater in western Europe. - Agriculture, Ecosystems \& Environment 26, 189-214.

THRÄN, D. (2005). Nachhaltige Biomassenutzungsstrategien im europäischen Kontext: Analyse im Spannungsfeld nationaler Vorgaben und der Konkurrenz zwischen festen, flüssigen und gasförmigen Bioenergieträgern. - Institut für Energetik und Umwelt, Leipzig.

TraUpmanN, P. (2004). Anleitung zur Anlage von Kurzumtriebsflächen. - FFP - ForstPlatte-Papier.

TrIPEPI, R. R., GeORGe, M. W., DumROese, R. K. \& WenNy, D. L. (1991). Birch seedling response to irrigation frequency and a hydrophilic polymer amendment in a container medium. - Journal of Environmental Horticulture 9, 119-123.

TSAKALDIMI, M. (1998). Effect of hydrophilic polymer on seedling survival and growth of evergreen broadleaved species. - Epistēmonikē Epetērida tou Tmēmatos Dasologies kai Fusikou Periballontos, Aristoteleio Panepistēmio Thessalonikēs 41, 1485-1496.

VAN DEN BURG, J. (1990). Foliar analysis for determination of tree nutrient status - a compilation of data. 2. Literature 1985-1989. - "De Dorschkamp", Institute for Forestry and Urban Ecology, Rapport Nr. 591, Wageningen, Niederlande.

VAN DEN DRIESSCHE, R. (1999). First-year growth response of four Populus trichocarpa $x$ Populus deltoides clones to fertilizer placement and level. - Can. J. For. Res. 29(5), 554-562 
VANDENBerghe, C., FrelÉChouX, F. \& Buttler, A. (2008). The influence of competition from herbaceous vegetation and shade on simulated browsing tolerance of coniferous and deciduous saplings. - Oikos 117, 415-423.

Vandenberghe, C., Freléchoux, F., Gadallah, F. \& Buttler, A. (2006). Competitive effects of herbaceous vegetation on tree seedling emergence, growth and survival: does gap size matter? - Journal of Vegetation Science 17, 481-488.

VIERO, P. W. M. \& LITTLE, K. A. (2006). A comparison of different planting methods, including hydrogels, and their effect on eucalypt survival and initial growth in South Africa. - Southern Forests: a Journal of Forest Science 208, 5-14.

VISNIIC, C. (2006). Aufforstung von sommertrockenen Standorten mit heimischen Baumarten in Bosnien. - Dissertation, Georg-August-Universität Göttingen.

Walle, I. V., Camp, N. V., Casteele, L. V., Verheyen, K. \& Lemeur, R. (2007). Shortrotation forestry of birch, maple, poplar and willow in Flanders (Belgium) I . Biomass production after 4 years of tree growth. - Biomass and Bioenergy 31, 267-275.

WeBER, G. \& BAHR, B. (2000). Eignung bayerischer Standorte für den Anbau von Esche (Fraxinus excelsior L.) und Bergahorn (Acer pseudoplatanus L.). - Forstw. Cbl. 119, 263-275.

WENK, M. (2007). Mäuse. - Waldschutz Merkblatt 53 Ministerium für Ländliche Entwicklung, Umwelt und Verbraucherschutz des Landes Brandenburg.

WILLOUGHBY, I. \& MCDONALD, H. G. (1999). Vegetation management in farm forestry: a comparison of alternative methods of inter-row management. - Forestry 72 , 109-122.

WITTWER, R. F., DOUGHeRTY, P. M. \& COSBY, D. (1986). Effects of Ripping and Herbicide Site Preparation Treatments on Loblolly Pine Seedling Growth and Survival. - Southern Journal of Applied Forestry 10, 253-257.

WOLF, H. \& BÖHNISCH, B. (2003). Anbau schnellwachsender Gehölze auf stillgelegten landwirtschaftlichen Flächen zur Holzstoffproduktion. - Tagungsband zum IBVSymposium "Produktvielfalt durch Ressourcenvielfalt - Potenziale genetischer Ressourcen" vom 23.-24. September 2003 in Bonn.

WOODHOUSE, J. M. \& JOHNSON, M. S. (1991). The effect of gel-forming polymers on seed germination and establishment. - Journal of arid environments 20, 375380 .

WU, J., LUO, Z., CHEN, Y. \& LIN, W. (2005). The effects of the different hydrogel substrates on seedling culture in Acacia mangium. - Journal of Nanjing Forestry University (Natural Sciences Edition) 29, 53-55.

YU, Q., TIGeRstedt, P. M. A. \& HaAPANeN, M. (2001). Growth and phenology of hybrid aspen clones (Populus tremula L. Populus tremuloides Michx.). - Silva Fennica 35, 15-25. 


\section{Lebenslauf}

\section{Persönliche Daten}

Name:

Geburtstag:

Geburtsort:

Staatsangehörigkeit:

Familienstand:
Bettina Stoll

17.06. 1981

Oldenburg (Oldb)

deutsch

ledig

\section{Anschrift}

General-von-Stein-Stein-Straße 7

85356 Freising

E-mail: Bettina.Stoll@gmx.de

\section{Schulausbildung und Studium}
$1987-2000$
Schulausbildung
$06 / 2000$
Abitur am Gymnasium Zwischenahn Edewecht, Bad Zwischenahn
$10 / 2000-09 / 2003$
Bachelor-Studium „Forstwissenschaften und Waldökologie" an der Georg-August-Universität Göttingen
Abschluss: Bachelor of Science
$10 / 2003-04 / 2006$
Master-Studium "Forstwissenschaften und Waldökologie", Schwerpunkt "Holzbiologie und Holztechnologie" an der Georg-August-Universität Göttingen
Abschluss: Master of Science
10/2006 - 12/2011 Promotionsstudium an der Fakultät für Forstwissenschaften und Waldökologie, Georg-August-Universität Göttingen 